\title{
Preparing for the Olympic Games: Training adaptation in endurance sports
}

Citation for published version (APA):

Rietjens, G. J. W. M. (2002). Preparing for the Olympic Games: Training adaptation in endurance sports. [Doctoral Thesis, Maastricht University]. Datawyse / Universitaire Pers Maastricht. https://doi.org/10.26481/dis.20020628gr

Document status and date:

Published: 01/01/2002

DOI:

10.26481/dis.20020628gr

Document Version:

Publisher's PDF, also known as Version of record

\section{Please check the document version of this publication:}

- A submitted manuscript is the version of the article upon submission and before peer-review. There can be important differences between the submitted version and the official published version of record.

People interested in the research are advised to contact the author for the final version of the publication, or visit the DOI to the publisher's website.

- The final author version and the galley proof are versions of the publication after peer review.

- The final published version features the final layout of the paper including the volume, issue and page numbers.

Link to publication

\footnotetext{
General rights rights.

- You may freely distribute the URL identifying the publication in the public portal. please follow below link for the End User Agreement:

www.umlib.nl/taverne-license

Take down policy

If you believe that this document breaches copyright please contact us at:

repository@maastrichtuniversity.nl

providing details and we will investigate your claim.
}

Copyright and moral rights for the publications made accessible in the public portal are retained by the authors and/or other copyright owners and it is a condition of accessing publications that users recognise and abide by the legal requirements associated with these

- Users may download and print one copy of any publication from the public portal for the purpose of private study or research.

- You may not further distribute the material or use it for any profit-making activity or commercial gain

If the publication is distributed under the terms of Article $25 \mathrm{fa}$ of the Dutch Copyright Act, indicated by the "Taverne" license above, 


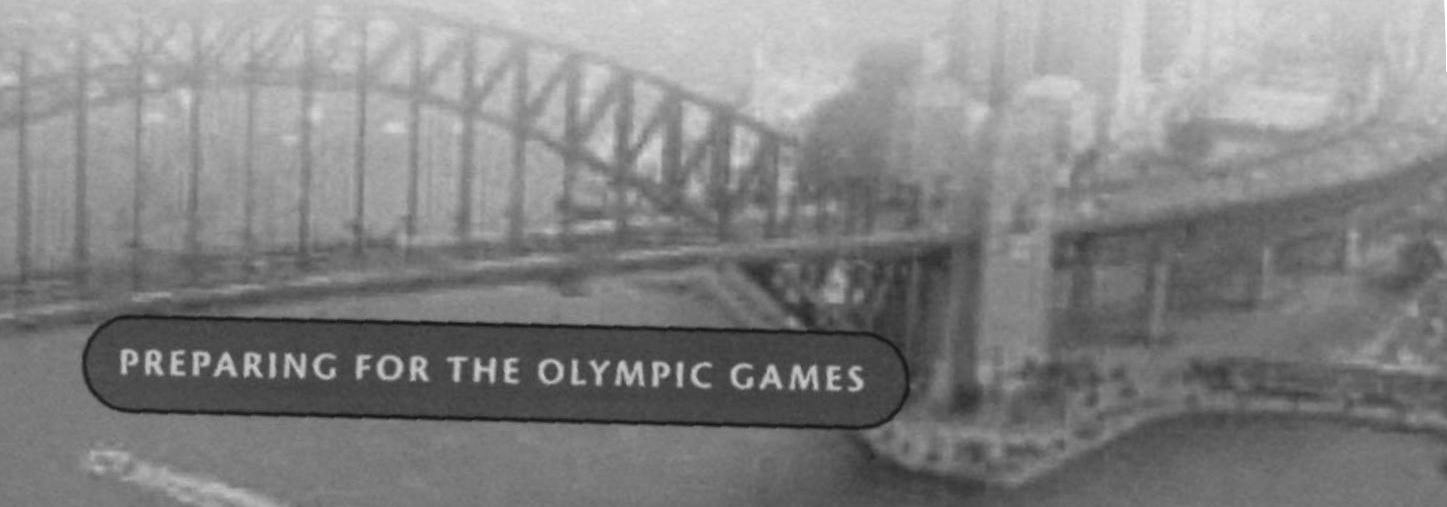

Training Adaptation in Endurance Sports

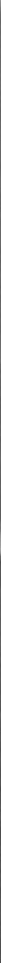




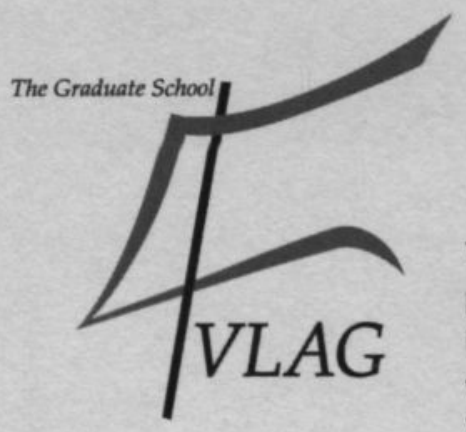

The study presented in this thesis was performed at the Nutrition and Toxicology Research Institute Maastricht (NUTRIM), which participates in the Graduate School VLAG (Food Technology, Agrobiotechnology, Nutrition and Healt Sciences) accredited by
the Royal Netherlands Academy of Arts and
Sciences

(c) Gerard Rietjens, Maastricht 2002

ISBN 90-9015889-8

Design and Lay-out:

Jossie Reijners

Printing agency:

Datawyse / Universitaire Pers Maastricht 


\section{PREPARING FOR THE OLYMPIC GAMES}

Training Adaptation in Endurance Sports

\section{PROEFSCHRIFT}

ter verkrijging van de graad van doctor aan de Universiteit Maastricht, op gezag van de Rector Magnificus, Prof. Dr. AC Nieuwenhuijzen Kruseman, volgens het besluit van het College van Decanen, in het openbaar te verdedigen op vrijdag 28 juni 2002 om 16.00 uur

door

Gerardus Joannes Wilhelmus Mechtildis Rietjens

Geboren te Weert, 22 juni 1963 


\section{Promotores:}

Prof. Dr. H. Kuipers

Prof. Dr. Ir. W.H.M. Saris

\section{Co-promotor}

Dr. H.A. Keizer

\section{Beoordelingscommissie/ assessment committee}

Prof. Dr. K. Westerterp (voorzitter)

Dr. R.J. Brummer

Prof. Dr. J. Steinacker (University of Ulm, Germany)

Prof. Dr. A.H. Viru (Tartu University, Estonia)

Prof. Dr. G.J. van der Vusse

Financial support by the Netherlands Olympic Committee * Netherlands Sport Federation (NOC*NSF) for the publication of this thesis is gratefully acknowledged.

Additional financial support was granted by:

E. Jaeger GmbH (Germany) , Instruchemie BV, Medith BV, Medische Laboratoria Dr. Stein en Collegae, Lode BV, Medifit 
Knowing is not enough; we need to apply.

Willing is not enough; we need to do.

\section{Goethe}

Pap en Mam 


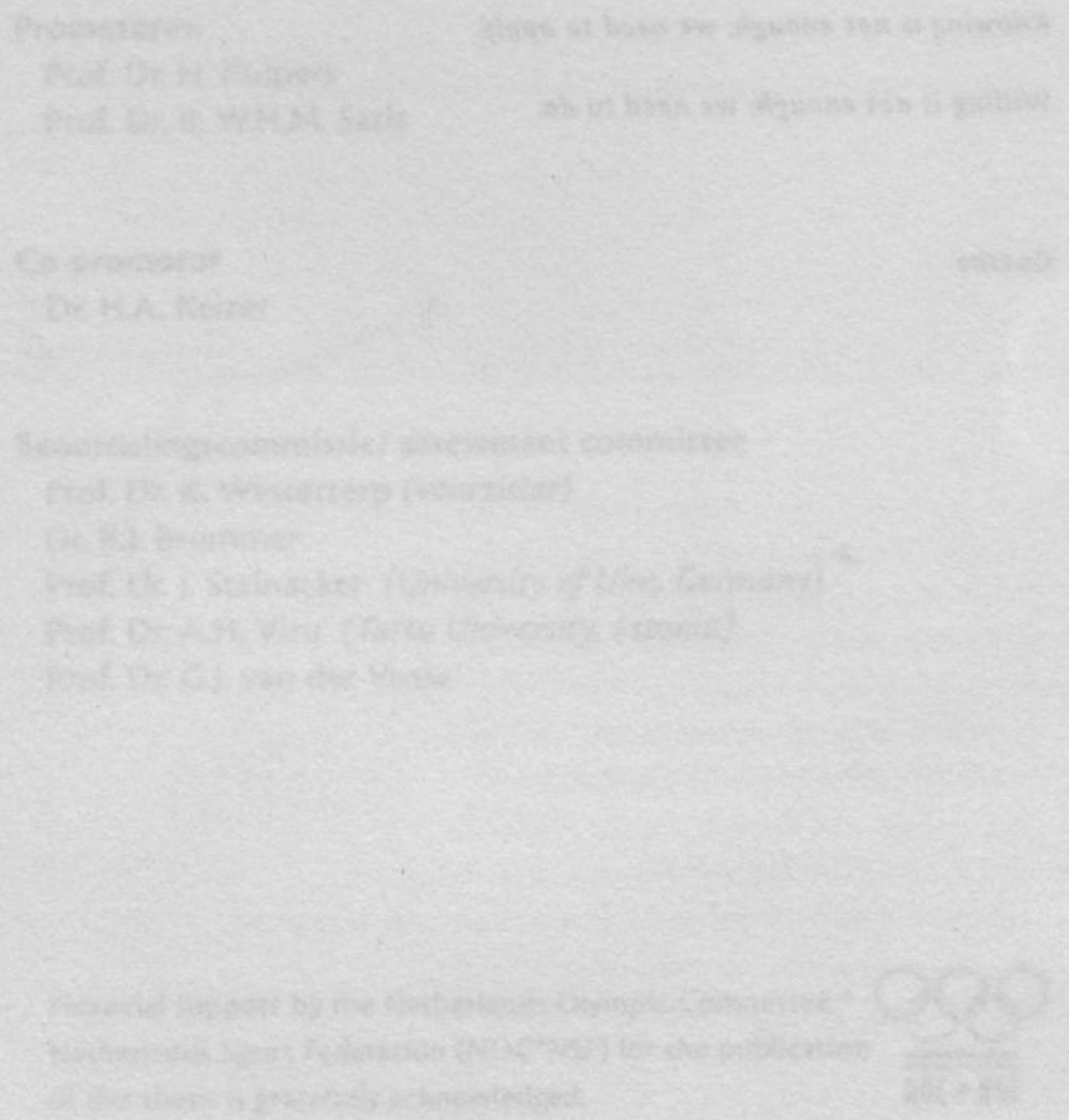

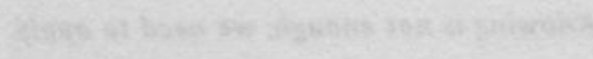

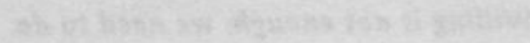




\section{CONTENTS}

\section{CHAPTER:}

1 General introduction 9

2 Physiological characteristics of elite Olympic distance 37 triathletes

3 Red blood cell profile of elite Olympic distance triathletes; A three-year follow up

4 Hormonal changes throughout the year in elite male and female triathletes; $A$ one year survey

5 Physiological variables and lactate indices in incremental exercise tests with different stage durantion

6 Effects of stage duration in incremental running tests, effects on physiological variables

7 A reduction in training volume and intensity during 21-days does not impair performance in cyclists

8 Physiological, biochemical and psychological markers for overreaching; Early Markers for overreaching

9 Physiological and psychological variables respond different in competitive cyclists and runners after a period of intensified training

10 General Discussion

Summary

Samenvatting

Dankwoord 


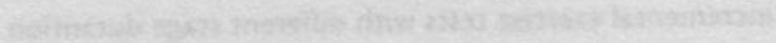

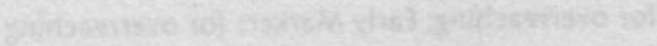


CHAPTER 1

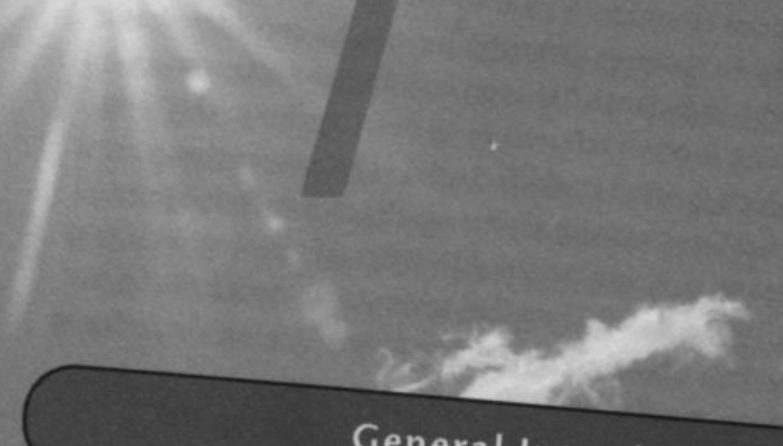

General Introduction

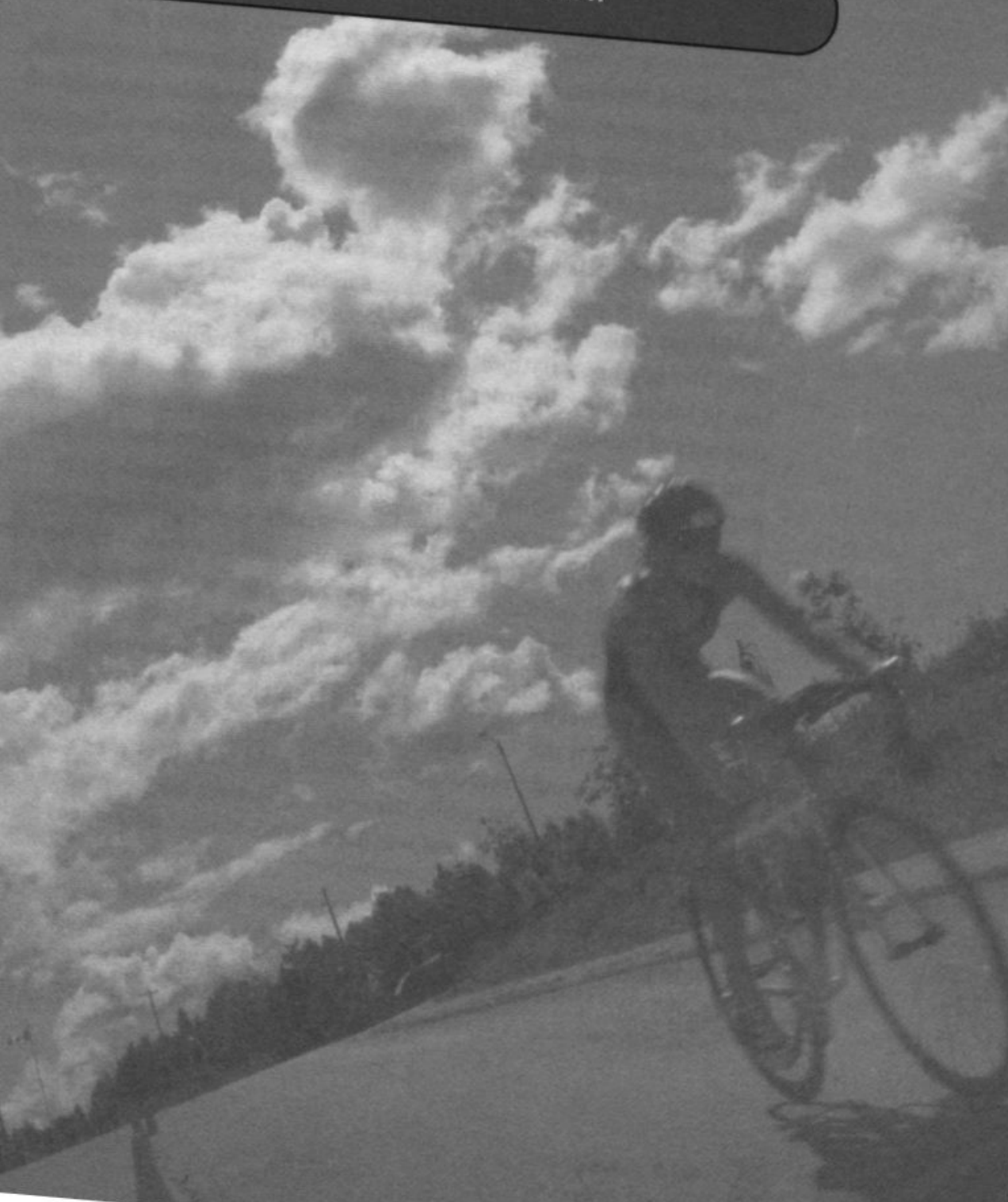


This chapter provides an overview of training preparation and the most common methods used to measure training adaptation and performance in sports practice. Special emphasis is paid to the delicate balance between training and overtraining. Overtraining can be considered as a short-term or long-term imbalance between training and adaptation. Short term over-training is often referred to as over-reaching, and long term overtraining, which is often multi factorial, is known as the overtraining syndrome. Overtraining always results in a drop in sport performance, and clearly is an unwanted side effect of training, as it may ruin a whole competitive season. Therefore several attempts have been made to identify markers of early overtraining (overreaching), in order to prevent an overtraining syndrome.

In the first chapter several markers used to monitor training and adaptation are described, with emphasis on the involvement of the neuroendocrine system. Also other markers such as hematological and psychological markers are addressed. This chapter ends with the specific aims of the present study. Furthermore, an overview will be provided of the studies described in the following chapters of this thesis.

\section{Olympic triathlon}

Triathlon is a modern sport that has been declared an Olympic sport since 1994. The Olympic triathlon consists of $1.5 \mathrm{~km}$ swimming, $40 \mathrm{~km}$ cycling, and $10 \mathrm{~km}$ running. In order to be able to compete at elite level large amounts of training have to be done. The primary goal of athletic training is to enhance performance and to peak at the right moment. To push the performance capacity to its upper limit relatively high amounts of intensive exercise are assumed to be necessary. Consequently, athletes often balance on the edge between training and overtraining. Therefore, one of the most difficult parts of the training process is to find the optimal balance between training and adequate recovery. A correct balance between training and recovery is of utmost importance, since the difference between winning and losing is small. Snyder and Foster (1994) reported that in the 1988 Olympic speed skating event in Calgary the difference in average velocity between all gold and silver medal performances was $0.3 \%$, while the mean difference between all the gold medallists and the 4 th places was $1.3 \%$ (106). Similar differences can be found in other sports.

Unfortunately few scientific data exist about the optimal amount of training for peak performance. Based on a few studies there appears to be an inverted $U$-shaped relationship between training volume and increase in performance. It is assumed that there is an optimal amount of training, 
which will yield an optimal performance. However, this optimal amount of training is poorly defined, and passing this 'gray' area may lead to overtraining.

The general theme of this thesis is to investigate whether indicators can be identified to diagnose overtraining in an early stage in order to prevent overtraining.

It is common practice to monitor the training process and the health status of athletes by taking repeated blood samples and exercise tests. However, the efficiency of these measures is not very well documented. Therefore, another aspect of the present thesis is to evaluate blood tests and exercise tests, so coaches can be provided with practical advise.

\section{Principles of training adaptation}

Although little is known of the relationship between the volume of training and training effect, it is assumed to be characterized by an inverted $U$-shaped curve (Figure 1$)(63)$. Since the top of this $U$-curve is poorly defined, the balance between training and recovery is a delicate one. The best training provides a level of stress yielding optimal physiological improvements without exceeding the athlete's tolerance. In sports science and sports practice most training models are based on the idea that physical exercise results in a disturbance of various cellular processes (121). In order to adapt to the physical strain, these

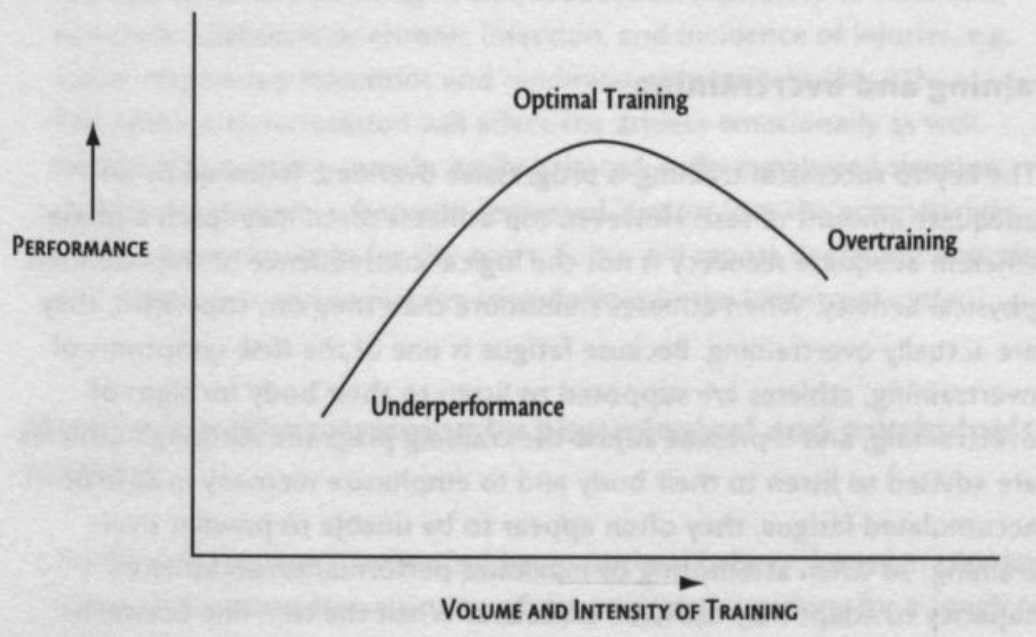

\section{FIGURE 1}

The inverted $U$-shaped chart, representing a schematic relationship between training and performance (adapted from Kreider, 1998 (61)) 
disturbances on cellular or sub-cellular level are assumed to trigger recovery processes, reflected in renewal of proteins, synthesis of enzymes, and the supply of building materials.

It is assumed that these recovery processes do not stop when the traininginduced disturbances are restored but do continue until a certain level of overcompensation is attained; this is referred to as supercompensation (Figure 2$)(63,121)$. Following the hypothesis that increasing training load on successive days of training (as visualized by the accumulation of black rectangles in fig 2) will enhance recovery, a gradual increase in performance (supercompensation) might be expected.

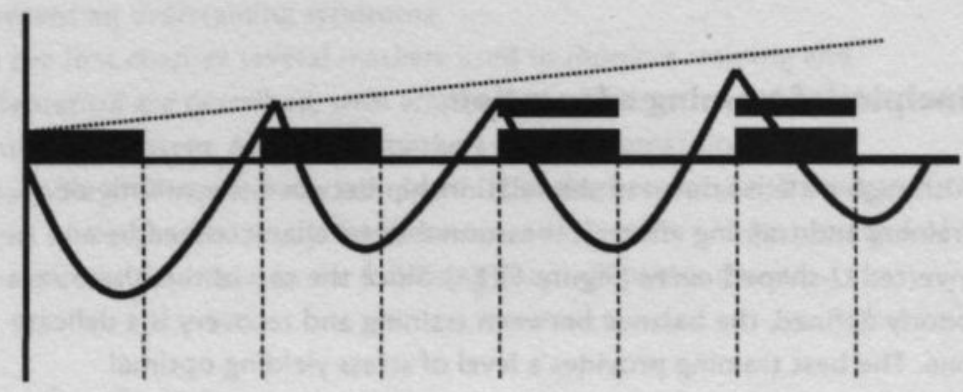

FIGURE 2

Schematic overview of super-/overcompensation (adapted from Bosboom, 1985 (11))

\section{Training and overtraining}

The key to successful training is progressive overload, followed by an adequate amount of rest. However, top athletes often may reach a phase wherein adequate recovery is not the logical consequence of the attended physical activity. When athletes train more than they can cope with, they are actually overtraining. Because fatigue is one of the first symptoms of overtraining, athletes are supposed to listen to their body for signs of overtraining, and if present adjust the training program. Although athletes are advised to listen to their body and to emphasize recovery in case of accumulated fatigue, they often appear to be unable to prevent overtraining. So when attempting to maximize performance, an athlete's capacity to adapt may easily be exceeded. When the thin line between adequate recovery and overtraining is crossed the athlete's performance will decline (44). After a single training session of adequate duration an intensity, the athlete's capacity to perform is severely reduced. However, 
adequate rest usually restores exercise performance. Often athletes train hard for some days in a row, in order to provide a stronger training and adaptation stimulus. This is referred to as reaching. However, when recovery after a period of hard training does not result in restoration of performance but rather results in a deterioration of performance, this is called overreaching. In case of overreaching some extra days of rest will usually restore performance capacity. The main symptom of overreaching is early fatigue during exercise. When exercise and recovery are not in balance over a longer period of time, more symptoms add to fatigue and this may result in an overtraining syndrome. An overtraining syndrome is characterized by increased fatigability, decline in sport performance, mental and mood changes, and changes in the neuro-endocrine system $(5,64,68-71,81,82)$.

The 'overtraining syndrome' is featured by a collection of emotional, behavioral, and physical symptoms that may persist from weeks up to several months. Amongst the physical symptoms of an overtraining syndrome, fatigue and chronic tiredness, are the most common ones and, although mostly retrospectively diagnosed, the first to appear. The chronic fatigue has to be sufficient to be debilitating to the athlete and must have persisted for longer than two weeks in the absence of any other identifiable illness. It will limit workouts and may even be present at rest, due to which, the athlete suffers an inability to meet his performance criteria. Blood chemical evaluation of overtrained athletes revealed reduced glutamine levels, elevated catecholamines and cortisol $(49,63)$. These alterations have been reported to suppress various indices of immune function, resulting in an increased susceptibility to infection, recurrent infections or chronic infection, and incidence of injuries, e.g. upper respiratory infections and tendinitis, respectively, $(86,92)$. The physical deterioration will affect the athlete emotionally as well. $\mathrm{He} /$ she may become moody, easily irritated, suffering altered sleeping patterns or insomnia, become depressed, and/or lose the competitive thrive and enthusiasm for the sport. Some will report decreased appetite and weight loss and in females irregularities in the menstrual cycle.

\section{Monitoring (Over)training by physiological and psychological markers}

Training adaptation is enhanced by an optimal balance between training load and recovery. However one of the toughest questions for a coach and athlete is to find out when to train harder and when to take a rest in order to reach the optimal performance level. Being able to identify this is an important consideration in relation to optimal athletic performance. With 
respect to this coaches and athletes generally rely on their intuition and feeling. In the last decades, coaches, athletes and sport scientists have tried to find more objective, reliable and valid tools to monitor the precise balance between rest and physical exercise.

One of the means to monitor training is conducting regular exercise tests in the laboratory and in the field. Exercise tests yield parameters of performance such as maximal power output, maximal running speed, maximal oxygen uptake $\left(\mathrm{VO}_{2} \mathrm{max}\right)$, and the plasma lactate response to exercise. Plasma lactate has received much attention during the last decades, and lactate measurements have been used on a large scale for performance evaluation. In addition hormones as well as psychological markers have been used to monitor overtraining. Some of these variables are briefly reviewed in the following section.

\section{Performance}

Although it seems reasonable to assume that competition is the ultimate test to assess the preceding training efforts, it does not reveal the adaptation of the various physiological systems involved in exercise. Therefore, several laboratory tests have been developed that resemble competition but lack the major drawback of competitions: poor reproducibility and poor control. For this purpose several exercise protocols have been developed and the most accepted ones are amongst others: the time to exhaustion, the maximal power output and the time trial. During a time to exhaustion protocol, subjects have to exercise at a certain percentage of their maximal performance until exhaustion.

\section{Performance testing as a diagnostic tool for overtraining}

In all definitions of overtraining, decreased performance is an everrecurring variable. Therefore it seems that the best and most important indicator for overtraining is a decrease in performance $(62,63,70)$. Fry and co-workers considered a performance drop of as little as $2 \%$ after a regeneration period as an overtraining response (31). This definition however, is difficult to apply, because Kuipers et al. found variability in maximal workload on a cycle ergometer of around $5 \%$ in trained subjects (65). A deterioration in performance due to overreaching must therefore be differentiated from the day-to-day fluctuation in performance. More discriminating is the definition of O'Conner (1997): 'A reduction in performance from $5 \%$ or greater for an extended period for 2 weeks or longer that occurs during or following a period of overreaching and fails to improve in response to short-term reductions in training' (87). Hooper et al (1995) found that performance decrements ranging from 0.7 to $15 \%$ were clearly the result of overtraining (45). Fry et al. (1992) concluded also 
that the magnitude of a decreased performance necessary for the diagnosis of overreaching and the nature of an appropriate regeneration period are difficult to define. Both may vary depending upon the training background of the subjects and the nature of the preceding training (31). Although a decrease in performance is said to be the most important determinant of overtraining, Snyder et al. (1995) failed to find major effects on the performances in a time trial in cyclists which were all classified as overreached (107). From these studies it can be concluded that a drop in performance seems to be a strong indicator for overtraining but there is still some conflicting data on this point.

\section{Maximal Oxygen uptake}

Another variable that is often used as a measure for performance is the maximal oxygen uptake. The maximal oxygen uptake can be defined as the highest rate of oxygen uptake and utilization by the body during severe exercise. Maximal oxygen uptake is largely genetic defined. However by endurance training an increase up to $20 \%$ in maximal oxygen uptake is found (43). It is generally accepted that maximal oxygen uptake is a good indicator of endurance performance. However there are some reservations. First, in a study of Kuipers et al. it was found that there may be a considerable intra-variability in maximal oxygen uptake (65). Second, it was found in well-trained athletes that maximal performance can increase without an increase in maximal oxygen uptake (3).

\section{Lactate}

The energy required for normal cell function is predominantly obtained from adenosine triphosphate (ATP), which is normally produced by oxidation of glucose (aerobic metabolism). However, if not enough oxygen is available for this process the cell ATP production takes place during anaerobic metabolism with production of pyruvate. Pyruvate is converted intra-cellulary to lactate and is transported out of the cell into the blood. In healthy adults at rest, the lactate concentration in the blood is $<1.5 \mathrm{mmol} / \mathrm{l}$ ( $13.5 \mathrm{mg} / \mathrm{dl}$ ), but it can increase significantly during physical exercise. In athletes peak lactate levels up to $15-17 \mathrm{mmol} / \mathrm{l}$ have been reported.

Wassermann and Mcllroy described in 1964 the existence of a critical threshold at which the need for oxygen in the working muscle exceeds the availability of oxygen resulting in an increase of the anaerobic metabolism (123). In the last couple of years several studies reported evidence that besides a limited availability of oxygen, more factors contribute to the formation of lactate and that lactate can be formed under conditions that are fully aerobic $(13,20)$.

Lactate accumulation in the blood depends not only on the production in 
the muscle cell but also on the exchange across the cell membrane and the consumption of lactate in the neighboring cells. Until recently it was assumed that lactate crossed the cell membrane by simple diffusion.

Elegant studies by Bonen's group have shown that at least a large part of the lactate transport across the cell membrane occurs via monocarboxylate transporters (MCT) (9). Till now seven different lactate transporters have been described ( 8 ). It has become clear that the working muscle not only produces lactate but also uses it as a fuel. Important lactate consumers are the heart muscle and the slow twitch muscle fibers (34). Furthermore, lactate is a precursor for liver gluconeogenesis (91). In the present study exercise testing and the lactate response to exercise have been investigated (Chapter 5 and 6).

\section{Lactate as a diagnostic tool for overtraining}

Decreased lactate values in response to sub maximal and maximal exercise intensity have been suggested as a marker for overtraining by some authors $(46,47,67,112)$

A decreased sympathetic drive, reduced catacholamine sensitivity and decreased glycogen stores have all been hypothesized to explain the decreased lactate response to exercise during overtraining $(22,55,67)$. However, the problem with using lactate as marker for overtraining is that decreased lactate to a given exercise intensity also reflects a normal adaptation to endurance training and improvement of the physical performance.

\section{Hormones}

The basic training model assumes that the trigger for training adaptation is provided by the training-induced disturbed homeostasis. However, there is also some evidence that the recovery and adaptation processes are influenced by several hormones (122). Any stress, including acute physical exercise of moderate to high intensity is able to elicit marked changes in stress hormone secretions. These increments in plasma stress hormone levels are necessary to augment muscle enzyme activities (and consequently energy release and expenditure) manifold (16). In the recovery phase, termination of the stress response must occur in order to adapt to a higher level or to habituate or adapt to the same stress. Therefore several hormones are used for monitoring training adaptation, and to investigate their usefulness for early detection of overtraining (Chapters 4 and 8). To provide some background for hormone monitoring an overview of some of the most important hormone systems is given. 


\section{The hypothalamic-pituitary-growth hormone (HPGH) axis}

$\mathrm{GH}$ is an important metabolic hormone, which stimulates net protein anabolism, lipolysis, and linear bone growth. The cellular effects of $\mathrm{GH}$ are mediated directly and in many cases indirectly by their autocrine action upon the target cells. In this situation $\mathrm{GH}$ induces the release of insulinlike growth factor 1 and 2 (IGF-1, IGF-2). GH stimulates IGF-1 production in, among others, liver, fibroblasts and skeletal muscle. $\mathrm{GH}$ has insulin-like effects (increased glucose and amino acid uptake by target cells) and antiinsulin effects. In healthy subjects $\mathrm{GH}$ administration stimulates muscle protein synthesis almost instantaneously (33) but not whole body protein synthesis (32).

Growth hormone (GH) secretion is age dependent (29). It is maximal during puberty and early adolescence; thereafter $\mathrm{GH}$ secretion gradually declines to much lower levels (94). Under basal conditions, growth hormone $(\mathrm{GH})$ is secreted in an intermittent, pulsatile pattern by the anterior pituitary and also shows a diurnal rhythm (95). Daytime levels of growth hormone were reported to be very low (actually below the detection limits of the assays), with no pulsatations. With sleep onset and coinciding with the initial periods of slow wave sleep, distinct bursts of $\mathrm{GH}$ secretion occurs (110).

The pituitary release of $\mathrm{GH}$ into the bloodstream is increased by hypothalamic growth hormone releasing-hormone $(\mathrm{GHRH})$ and decreased by somatostatin or somatotropin-release-inhibiting hormone (SRIH). The rhythmic pulsatations of GHRH and SRIH each have a periodicity of 3-4 hr, and are $180^{\circ}$ out of phase (111). The amount of GHRH released deter-mines the $\mathrm{GH}$ amplitude, while SRIH determines the frequency and dura-tion of the $\mathrm{GH}$ bursts (60). At last, peripheral afferent signals may be important modulators of $\mathrm{GH}$ secretion and/or action since no effect of growth hormone on space flight-induced muscle atrophy could be found(48).

\section{Exercise and the growth hormone}

Acute exercise increases $\mathrm{GH}$ secretion in an intensity dependent manner (84), albeit that a wide inter individual variation exists (84). This would imply the more anaerobic the work, the more GH increases (116), which was observed after heavy resistance training (59). During long-term endurance exercise plasma $\mathrm{GH}$ reaches peak values before the end of the exercise bout, and levels off thereafter or eventually declines. After exercise $\mathrm{GH}$ levels normalize within 1-2 hours.

\section{The hypothalamic-pituitary-thyroid (HPT) axis}

Thyroid hormones have important effects on almost all tissue, including skeletal muscle. They modulate thermogenesis, protein-, carbohydrateand lipid metabolism. Increased thyroid hormone stimulated calorigenesis 
is reflected in increased oxygen consumption of most tissues. Protein synthesis in skeletal muscle is enhanced at moderate increments of plasma T4. The thyroid gland is stimulated to produce and release thyroxine (T4) and the metabolically more active 3,4,3'-triidothyronine (T3) by thyroid stimulating hormone (TSH) released from the anterior pituitary. TSH production is stimulated by thyrotropin-releasing hormone (TRH), synthesized in the paraventricular nuclei of the hypothalamus. Thyroid hormones (T4 and T3) exert negative feedback at the hypothalamus and at the pituitary to regulate TRH and TSH levels (83). Like other anterior pituitary hormones, serum TSH displays both episodic (pulsatile) and circadian variations. In healthy subjects, the mean TSH pulse frequency is approximately 9-12 per 24 hours (37). The 24-hour secretion pattern shows a distinct circadian rhythm, with increases in TSH pulse amplitude at night. Thyroid function is influenced by a variety of other hormones, including ACTH and glucocorticoids.

\section{Exercise and thyroid secretion}

Acute exercise is able to stimulate the hypothalamic-pituitary-thyroid axis almost instantaneously (78) as observed from the increase in thyroid stimulating hormone (TSH). This will result in an increased conversion of T4 to T3. Hackney et al., (41) evaluated the change in nocturnal concentrations of $\mathrm{T} 4$ after exercise ( $90 \mathrm{~min}, 70 \% \mathrm{VO}_{2} \mathrm{max}$ ) compared with a control day and found a significant augmentation of T4. More recently, Hackney and Gulledge (40) found only transiently increased plasma T3 level directly after acute exercise.

Boyden and Pamenter, investigated the effects of endurance training on the thyroid axis of 17 women (12). The training was increased from 0 to 50 miles/week within a year. The authors found an increased resting TSH level, which paralleled the increase in mileage. However, the TRH stimulated TSH response was exaggerated at 30 miles/week, but was diminished at 50 miles/week. This suggests that the amount of training stress changes the conversion of T4 to T3.

\section{The hypothalamic-pituitary-adrenal (HPA) axis}

The HPA axis, together with the autonomic nerve system, is the most important 'stress' system of the body. Once a stressor (physical exercise, psychic stress) has exceeded a certain threshold, a systemic reaction takes place that includes the brain and peripheral components, the HPA axis and the sympathetic nervous system. The central stress system includes the corticotropine releasing hormone ( $\mathrm{CRH}$ ) neurones, located in the hypothalamic paraventricular nucleus and other brain areas, and the locus ceruleus and other central sympathetic systems in the brainstem (19). The secretions of corticotrophines include adrenocorticotropic hormone $(\mathrm{ACTH})$ and B-endorphin. 
$\mathrm{CRH}, \mathrm{ACTH}$ and consequently cortisol are secreted in a pulsatile and diurnal pattern. The ACTH pulse frequency is rather constant during night and day, the pulse amplitude, however, varies. The secretory bursts increase in amplitude after 3-5 hours of sleep, reach a maximum the last few hours before and about one hour after awakening, decline thereafter and are minimal in the evening (118). Accordingly, plasma cortisol values are highest at awakening, are low in the afternoon and evening to reach nadir values during the first two non-REM/REM sleep cycles but rise markedly during the second half of sleep (10). Meals (especially those with high protein content) at lunch- and sometimes at dinnertime, cause additional secretory episodes (104).

Results from a study by Kjaer et al., (56) indicated that afferent nervous activity from working muscles stimulated the secretion of ACTH and B-endorphin. The authors also found that this was not true for $\mathrm{GH}$, catecholamines, and insulin. They argued that the direct, feed forward, stimulation of neuro-endocrine centers from motor centers in the brain is more important than the nervous feedback in these cases.

\section{Exercise and HPA hormone secretion}

Acute exercise of sufficient intensity (at least $70 \% \mathrm{VO}_{2} \mathrm{max}$ ) significantly increases plasma $\mathrm{ACTH}$, cortisol and B-endorphin levels in an intensity dependent manner $(28,50,58)$. With very high intensities (i.e. $120 \%$ $\mathrm{VO}_{2}$ max), ACTH was already increased after 1-minute exercise and cortisol increments were significant after a time delay i.e. after 15 minute of recovery, suggesting an increased $\mathrm{ACTH}$-induced steroidogenesis (15). Increasingly elevated plasma $B$-endorphin levels are found after $100 \mathrm{~m}$, $1500 \mathrm{~m}$ and $10.000 \mathrm{~m}$ running events (93), indicating that both exercise intensity and duration are important.

After physical training the ACTH response to a standard sub-maximal exercise is significantly suppressed $(51,58)$, albeit that at the same relative workload, ACTH levels were relatively more increased after training (51). In response to $\mathrm{CRH}$ stimulation, highly trained subjects had blunted $\mathrm{ACTH}$ and cortisol responses, resembling a state of hypercortisolism $(74,75)$. However, Chrousos (19) argued that in these cases other non-CRH factors play a more important role in the activation of the HPA axis by exercise. He suggested that vasopressin produced by the parvocellular component of the paraventricular nucleus might be a good candidate.

Work in rats showed that exercise training attenuated $A C T H$ responses for heterologic stressors as well, suggesting that cross-adaptation occurred $(90,124)$. Whether this occurs in athletes as well, is not known.

The primary action of ACTH on the adrenal cortex is to increase cortisol secretion by increasing its synthesis, but the hormone also increases the LDL-receptor, which affects steroid synthesis (89). Extra adrenal effects of 
$\mathrm{ACTH}$ involve the increase in glucose and amino-acid uptake by skeletal muscle and stimulation of lipolysis in the adipocyte (66).

\section{The hypothalamic-pituitary-gonadal (HPG) axis}

The gonads are stimulated to synthesize and secrete androgens, estrogens and progestins by the concerted action of luteinizing hormone (LH) and follicle stimulating hormone (FSH). The anterior pituitary gonadotrophs are under control of gonadotropin releasing hormone $(\mathrm{GnRH})$. The HPG axis is regulated by the feed back effects of gonadal hormones (estrogens, progestagens and androgens). Since steroid receptors are found in many parts of the brain (98), gonadal hormones regulate sexuality, but influence mood state, as well. Gonadotropins are secreted in a rhythmic, pulsatile fashion. This is for the greater part caused by the hypothalamic pulse generator $\mathrm{GnRH}$ (57). Following the rhythmic $\mathrm{GnRH}$ discharges, $\mathrm{LH}$ is secreted in bursts approximately every 90-120 min. $\mathrm{GnRH}$ rhythmicity varies to some extent according to the endocrine milieu (57). Testosterone and progesterone decrease the frequency. In response to estrogens, normal women release $\mathrm{LH}$, whereas men do not. In the scope of this study only the function of testosterone will be discussed.

\section{Exercise and HPG hormone secretion}

Acute exercise in female athletes has been found to increase plasma testosterone, probably irrespective of the exercise duration and phase of the menstrual cycle (50). In men, acute exercise of different modalities (running, weight training, cycling) is able to increase plasma testosterone levels, with the exception of a maximal tethered swim of 14 minutes, where a consistent decrease in plasma testosterone levels have been observed (23). Unlike in female athletes, in male athletes testosterone levels during prolonged exercise ( $>60$ minutes) may increase during the first hour, but decrease when the exercise continues. During the recovery phase, this decline may continue and result in plasma testosterone levels well beyond the pre-exercise values (25-50\%). Prolonged exercise to exhaustion may have pronounced effects on nocturnal hormone secretions, and consequently the anabolic influence of sleep. Kern and colleagues (54) found in triathletes that $120-150 \mathrm{~km}$ bicycle exercise (causing an 'overwhelming distress') was able to decrease plasma testosterone during the whole night but increase cortisol levels during the first half and decreased cortisol secretion during the second half of sleep, as compared to the non-exercise control or the low distressing exercise day. However, plasma gonadal hormone changes are in most cases very transitory, i.e. return to a normal range occurs within hours to a couple of days after exhaustive exercise. The mechanisms behind the decreasing testosterone levels seem to differ somewhat between males and females. 
The decreases in testosterone in the male athlete may be explained (among others) by catecholamine-induced decrease in testicular blood flow. In the female athlete an increased adrenal secretion is probably involved (50), whereas a decreased metabolic clearance rate may also be the case (52). Effects of strenuous training, particularly endurance training, on the HPG axis have been extensively studied in women (see for reviews references 7 , 53).

The available data on the effects of strenuous training on plasma testosterone levels in male athletes are somewhat conflicting. Retrospective and prospective studies, either using isolated or serial blood sampling have shown that vigorous exercise training (running, cycling, rowing) may result in decreased resting free and total testosterone concentrations $(39,41,42,76)$.

\section{Actions of gonadal hormones}

Gonadal hormones exert many actions upon the body. From experiments in male and female rats, it appeared that testosterone increases skeletal muscle glycogen content and glucose uptake (114). During sub maximal exercise a glycogen sparing effect of testosterone was found in exercising female rats (115). Dohm and colleagues (24) found that the exerciseinduced decline in plasma testosterone levels was accompanied by increased excretion of products of muscle catabolism in rats. Although Allenberg and colleagues showed that testosterone might have some influence on glycogenesis, their data are (not yet) confirmed by other researchers (2). Therefore, it is unknown, whether the rather moderate decreases in testosterone levels as shown in sports practice have any effect on muscle metabolism.

\section{Involvement of the neuro-endocrine system in overtraining}

From the previous paragraphs it might be clear, that the neuro-endocrine system is involved in all kinds of stress, including physical exercise. Dysregulation of the HPGH, HPT, HPA and HPG axes has indeed profound influence upon mood-state, muscle metabolism and muscle contraction, given that a severe hormonal deficit occurs.

The first evidence for the involvement of the neuro-endocrine system in overtraining was provided by results reported by Barron and colleagues, (5). Although based on only four subjects (male marathon runners), who were diagnosed as suffering from an overtraining syndrome, their study offered for the first time some clues for the understanding of this syndrome. The authors found a blunted GH, ACTH and cortisol response after an insulin-induced hypoglycemia. Interestingly, responses to TRH and LHRH were normal, which proved (according to the authors) that the dysfunction was at the level of the hypothalamus. The exact contribution of training volume and/or intensity to the overtraining syndrome is, 
unfortunately, not possible, since precise information on training variables and possible other stressors was lacking in the study of Barron and colleagues.

Adlercreutz and colleagues (1) found that a decrease in the ratio of free testosterone (FT) to cortisol (C) correlated with an impairment in performance in runners. They hypothesized that a decreased FT/C ratio hampers the anabolic processes after exercise, and thus delay recovery. Although some investigators reported similar results, others were unable to confirm the validity of the FT/C ratio as an early marker in overtraining in various types of endurance- and strength athletes $(71,113,120,125)$. The discrepancy of these findings may be explained by several factors such as the resistance against stress of the HPG axis (99), and subtle changes occurring during sleep may be missed if one relies on only one blood sample $(41,54)$. In addition the normal diurnal rhythm may not be taken into account, since plasma testosterone levels are about $25 \%$ lower in the late afternoon and evening as compared to the values around noon. Thus, we agree with Urhausen and colleagues (112) that decrements in gonadal hormones more likely indicate the actual physiological strain of the previous training bout, rather than overtraining.

Besides the methodological problems of blood sampling to detect parameters for overtraining, the validity of cross-sectional studies can be questioned. In addition, in none of these studies precise information about the training intensity, volume and frequency was presented. However, Lehmann et al., (72) tried in a prospective study to dissociate between run training intensity and volume as causative factors for overtraining. In a period of 3 weeks, they increased either the amount of high intensity run training 2.5 fold in one group or, in another group, the weekly training volume increased about 2 fold. They found a decrease in resting and exercise plasma cortisol levels after the increased volume training only, whereas plasma testosterone and free testosterone did not change. The $\mathrm{CRH}$-induced plasma $\mathrm{ACTH}$ and cortisol responses were interesting. Compared to the overtrained condition, baseline ACTH levels and response on $\mathrm{CRH}$ was lower and cortisol response higher. The authors also postulated the hypothesis that similar to ACTH the resting levels of B-endorphin may be increased, which explains the inhibition of gonadotropin release.

In an attempt to evaluate the response of gonadal hormones, cortisol and B-endorphins on standardized exhaustive and non-exhaustive training Keizer et al (unpublished results) conducted a prospective study in 12 male runners. They were tested three times with an in-between interval of 8 days, i.e. after a light training period ( $\mathrm{LiTr}$ ), after a very intensive training period (ITr) and again after a light training/recovery period (RecTr). The core part of the training (warming-up and cool-down not included) 
consisted of 1 hour strictly standardized daily running with either low (continuous running at $60 \% \mathrm{VO}_{2}$ max in the LiTr and RecTr period) or bouts of high intensity $\left(95 \%-110 \% \mathrm{VO}_{2}\right.$ max alternated with $60 \% \mathrm{VO}_{2} \max$ in the ITr period). The results showed an unaltered $\mathrm{FT} / \mathrm{C}$ ratio after the intensive training period. In fact, the FT/C ratio increased because the plasma cortisol levels significantly decreased. Nevertheless, 5 out of 12 subjects had symptoms of overreaching (no training desire, impossibility to fulfill the training load, sleep disturbances, lethargy, and constant feelings of tiredness), but only two of them showed a decreased performance after ITr. It was striking that all 5 runners had severe problems with the light training program in the third period. There were slight decrements in plasma $B$-endorphin responses at exertion when the whole group was considered, but the overreached athletes showed a blunted B-endorphin response to standardized treadmill running after the heavy and distressing training week.

The mechanisms whereby the secretions of the various hypothalamicpituitary axes will be changed are difficult to reconcile. It cannot be denied that increments in circulating stress hormones affect the recovery rate and the duration of the recovery phase $(102,103)$. Summarizing the effects of strenuous exercise and training on the neuro-endocrine system and the involvement of this system in overreaching and overtraining is quite difficult, since the results of research devoted to this subject matter are far from unanimous, not at least since well conducted prospective studies for obvious reasons are scanty. However, mood-state changes, sleep disturbances, changes in recovery rate, prolonged feelings of fatigue, and changes in reproductive state all indicate maladaptation in certain parts in the brain with consequently changes in hypothalamic effector output. In depressed patients, and maybe also in overtrained athletes, either a decreased sensitivity for these neurotransmitters may have been occurred, or production rate may be impaired. In turn, this may explain the decreased $\mathrm{GH}, \mathrm{LH}, \mathrm{B}$-endorphin and TSH levels, since in general these neurotransmitters are excitatory. Additionally, lower $B$-endorphin levels may be involved in lower stimulation of $\mathrm{GH}$ release (79). Since these effects apparently take place during sleep, extended periods of blood sampling are mandatory in order to obtain more insight in training and overtraining-induced alterations of the neuro-endocrine system.

\section{Additional biochemical markers}

\section{Red and white blood profile}

In endurance sports total blood volume and its composition are a major determinant for performance $(36,108)$. For this reason, red and white 
blood cell profile are often routine tests in the sport (medical) practice and many scientific studies were done in this area. For instance, endurance performance depends largely on the oxygen supply to the working muscle, which is transported by red blood cells. A highly positive correlation between the number of circulating red blood cells and maximal oxygen uptake and exercise to exhaustion have been described $(27,35,85)$. The importance of a high red blood cell mass is well-known in sport practice and is emphasized by the increased use of blood doping, altitude training and more recently the use of recombinant human erythropoietin (17). Several studies in endurance athletes showed an increase in blood volume up to $20 \%$ and an increase in red blood cell mass as a normal reaction to endurance training whereas hemoglobin and hematocrit decrease due to the expanded plasma volume $(4,21,38)$. The decreased hemoglobin content as a result of increased blood volume is one of the numerous hypotheses that have been proposed to explain this so called 'sports anaemia' (26). Sports anemia is seen as a common problem in the sport practice, particularly in endurance sports. Other explanations for this anaemia are dietary iron deficiency, gastrointestinal and/or urinary tract bleeding and mechanical trauma of the red blood cell (foot-strike hemolysis) and bone marrow supression (see for reviews references $18,105,109$ ).

\section{Hematological variables in relation to overtraining}

Hematological changes such as low ferritin concentrations or decreased hemoglobin and hematocrit are associated with overreaching and overtraining $(69,70)$. Furthermore several reports on overtraining provide evidence of an increased susceptibility for infections. However, most studies done in this area failed to find any significant change and the relevance of these biochemical parameters is at least debatable.

Lehmann et al. (1997) tested the hypothesis that high-volume endurance training can be monitored using hematological and blood chemical parameters as markers of an early stage in the overtraining process (73). In that study WBC and Hct showed a significant decrease but they concluded that the diagnostic reliability of overtraining-related sensitive changes in blood-chemical parameters is a subject of discussion. In a study of Dressendorfer and co-workers a reduction in red blood cell count, hemoglobin concentration and hematocrit were found after doubling the training volume in marathon runners. Together with this decrease a significant increase in the total white blood cell concentration was found. However, all these variables were still within the normal physiological range (25). Ronsen et al. (2001) concluded that a doubling of the training and competition load during the competitive season compared to offseason did not alter the leukocyte and stress hormone response in elite Nordic skiers to an incremental exercise test to exhaustion (100). Among 
other hormones, ACTH and cortisol are known to be involved in the regulation of the concentration of circulatory leukocyte.

In conclusion, the interpretation of hematological data is confounded by unknown changes in plasma volume, because endurance training programs are generally thought to result in an overall expansion of plasma volume (101). If plasma volume changes are not accounted for, changes in red blood cell, hemoglobin and hematocrit have to be treated with care. Apart from this confounding variable, it is rather difficult to distinguish a training effect from an abnormal overreaching response based upon a red blood profile.

\section{Psychological markers}

Lately it has become clear that mood may be affected by exercise. Morgan reported that moderate intensity exercise might significantly decrease the so-called stress-related emotions such as anxiety (80). Mood may differ between athletes and sedentary people; that is, athletes are in a better mood than are non-athletes. Morgan previously reported that not only elite athletes, but also active individuals in general tend to score below the population average on various items of mood scales (81).

Several studies reported severe mood distortion after periods of intense training in well-trained endurance athletes $(81,82,97,117)$. The explanation for this apparent inconsistency is that mood improvements generally occur with moderate amounts of exercise, whereas disturbed mood is more frequent among endurance athletes who intensify their training. So stated simply, elite athletes get moodier when their training intensity or volume increases. Mood changes also appear to be observed in a study in well-trained horses by Bruin et al (14). These researchers observed changes in behavior such as reluctance to run, increased irritability, spontaneous biting, and refusal to eat (14). Mood alterations towards the negative side of the spectrum appear to be reversible, as has been observed in various studies $(81,82,97,119)$. Subsequent recovery during a tapering period was associated with a return towards baseline for all mood disorders.

\section{Profile of Mood State questionnaire}

From the previous paragraph it is clear that mood may be an important parameter in determining whether or not an athlete is suffering from overtraining. For this purpose McNair et al. developed the Profile of Mood States scale, a questionnaire consisting of sixty-five items (77). The POMS yields a global measure of mood, as well as measures of tension, anger, depression, fatigue and confusion, all negative mood states and the positive mood state vigor. The global measure of mood is retrieved by subtracting the positive mood state, vigor, from the sum of 
the five negative mood states and adding a constant factor of 100. Morgan et al. administered the Profile of Mood States (POMS) to approximately 200 men and 200 women, all of them members of the University swimming teams (81), as did Raglin et al. to a group of 84 female and 102 male swimmers and Berglund and co-workers to a small assembly of 14 world-class canoeists $(6,97)$. All researchers observed similar findings. One finding was that the higher the global or overall mood score, the worse the mood (88). They also agreed on the point that mood state disturbances increased in a dose-response manner as the training intensity or volume increased and that these factors decreased in accordance with the reduction in training stimulus. Raglin et al (1991) however, found an exception for tension, which appeared not to diminish in response to training reduction (97).

In his 1985 study Morgan described a so-called 'iceberg profile' for the elite athletes and the above average active individuals. This profile is called an 'iceberg profile' since the resulting configuration resembles an iceberg i.e. the five negative mood states of the POMS scale, tension, depression, anger, fatigue and confusion tend to score below the average score of the inactive population and on the positive mood state, vigour, athletes usually tend to score approximately one standard deviation above the population's mean. Finding the 'iceberg profiles' in swimmers however, is sometimes doomed to fail, since the mood of athletes during heavy training worsens and flattens the profile (88). Many researchers acquired the 'iceberg' findings and described an inverted 'iceberg profile' in athletes suffering from severe fatigue and reversible depression.

Morgan et al. was the first to describe that mental health is associated with high performance levels, whereas mood disturbances may be forerunners of a decline in performance. Predictions based on this theory have an accuracy of approximately $80 \%(80)$. Several investigators, therefore, suggested to monitor mood to prevent the possible negative effects of intensified training. Veale stated that the POMS appeared to be an excellent tool for this purpose (117).

According to Jeukendrup et al. the use of the Profile Of Mood State (POMS) questionnaire in a training study is rather unreliable, since filling out a 65 items containing questionnaire every day easily leads to less careful completion; moreover, participants would get bored filling out forms constantly (47). Berglund and co-workers, however instructed their subjects to be honest to themselves and assured them that the result of the POMS rating were not given to coaches unless the subject agreed to. He observed in the post-season evaluation that all participants used the POMS in order to increase or decrease their training load (6). According to O'Conner and Berglund the majority of athletes can change their attitude toward psychological monitoring of training.

In sum, it can be concluded that many studies brought up the successful 
use of psychological variables to trace overtrained athletes $(6,44,97)$.

\section{Psychological markers and overtraining}

In the present study the POMS was used in conjunction with other indicators in order to investigate its usefulness in the early detection of overreaching. Mood state measured by the POMS has successfully identified athletes showing signs of stress due to intensified training. An increase in training load is associated with a shift toward negative overall mood, and overall mood improves with reduction in training load $(30,81)$. However, there are no strict definitions in the literature for the magnitude of mood disturbance associated with overtraining. To classify athletes as overtrained some studies reported baseline POMS +1 SD as a risk level $(82,96)$. Morgan et al. (1987) used the POMS 'inverted iceberg profile' (81). Berglund and Säfström (1994) found out that athletes had a risk to develop overtraining if they showed an increase of global POMS of more than $50 \%$ over the individual basal off-season global POMS in combination with the sub scale fatigue being higher than the sub scale vigor (6). It is interesting to follow some sub scales because vigor and fatigue exhibit the largest change in response to overtraining where depression and anger appear to be one of the most effective markers of overreaching or overtraining $(81,97)$.

\section{Aim and outline of this thesis}

It is well known amongst athletes and coaches that in order to perform successfully the physiological training load has to be tremendous. However, it is the actual rest after exercise that gives the adaptation to a higher performance level. If sufficient rest is not included in a training program, regeneration from the training cannot occur and performance development is hampered. A persisting imbalance between excess training sessions and inadequate rest will ultimate result in a state of overreaching or overtraining. Because the scientific basis of training is still fragmentary, the art of training is to find the right balance between a sufficient amount of work and adequate rest.

A first aim of this thesis was to analyze variables and methods often used in sports practice in order to investigate their usefulness and reliability in predicting training ( $\mathrm{mal}$ ) adaptation in endurance sports and particularly in Olympic distance triathletes. Furthermore a second aim of this thesis was to find additional parameters that can indicate overtraining and that can provide information to the athlete in preventing him/her to reach the overtraining syndrome. 
Following the general introduction (Chapter 1), Chapter 2, Chapter 3 and Chapter 4 report some physiological, biochemical and hormonal characteristics of Olympic distance triathlon athletes, respectively. Over a period of three years male and female athletes were followed during their preparation towards the Sydney 2000 Olympic games. During this period several measurements were done in order to evaluate athletes training adaptation. This gave us the opportunity to follow the profile of some biochemical and physiological variables throughout a competition year in elite endurance athletes. Chapters 5 and 6, addresses the question which type of protocol is the most accurate to measure some key physiological variables for performance in cycling (Chapter 5 ) and running (Chapter 6). In addition, in this part of the study it was examined whether the respiratory frequency pattern during incremental exercise could be an alternative variable in measuring training adaptation. As stated before, eventually it is rest that makes the athlete perform better. However, many athletes and coaches are afraid that a reduction in training load results in loss in physiological condition and performance. Chapter $\mathbf{7}$ describes a study in which the effects of a 21-day reduction in training volume and training intensity on performance and performance related variables were investigated. In order to find potential markers for the early detection of overtraining, the behavior of physiological, biochemical, hormonal and psychological markers to a period of intensive training was investigated. This part of the study, described in Chapter 8. Chapter 9, deals with the question if runners and cyclists do respond in the same way to a period of intensive training. In the last chapter, Chapter 10, the results of the different studies are discussed and summarized, and general conclusions are drawn. Based on these conclusions some practical implications for the sport practice are provided. 


\section{References}

1. Adlercreutz H., M. Harkonen, K. Kuoppasalmi, H. Naveri, I. Huhtaniemi, H. Tikkanen, K. Remes, A. Dessypris, J. Karvonen. Effect of training on plasma anabolic and catabolic steroid hormones and their response during physical exercise. Int J Sports Med 7 Suppl 1: 27-28. 1986.

2. Allenberg K., N. Holmquist, S.G. Johnson, P. Bennet, J. Nielsen, H. Galbo, $N$.H. Secher. Effects of exercise and testosterone on the active form of glycogen sunthase in human skeletal muscle. In: Biochemistry of exercise, edited by H. G. Knuttgen, J. A. Vogel and J. Poortmans. Champaign: Human Kinetics, 1983.

3. Arts F.J., H. Kuipers. The relation between power output, oxygen uptake and heart rate in male athletes. Int J Sports Med 15: 228-231. 1994.

4. Balaban E.P., J.V. Cox, P. Snell, R.H. Vaughan, E.P. Frenkel. The frequency of anemia and iron deficiency in the runner. Med Sci Sports Exerc 21: 643-648. 1989.

5. Barron G.L., T.D. Noakes, W. Levy, C. Smith, R.P. Millar. Hypothalamic dysfunction in overtrained athletes. J Clin Endocronilogy and Metabolism 60: 803-806 1985.

6. Berglund B., H. Safstrom. Psychological monitoring and modulation of training load of world-class canoeists. Med Sci Sports Exerc 26: 1036-1040 1994.

7. Bonen A. Exercise-induced menstrual cycle changes. A functional, temporary adaptation to metabolic stress. Sports Med 17: 373-392 1994.

8. Bonen A. Lactate transporters (MCT proteins) in heart and skeletal muscles. Med Sci Sports Exerc 32: 778-789. 2000.

9. Bonen A., S.K. Baker, H. Hatta. Lactate transport and lactate transporters in skeletal muscle. Can J Appl Physiol 22: 531-552. 1997.

10. Born J., W. Kern, K. Bieber, G. Fehm-Wolfsdorf, M. Schiebe, H.L. Fehm. Night-time plasma cortisol secretion is associated with specific sleep stages. Biol Psychiatry 21: 1415-1424. 1986.

11. Bosboom H., F. Brouns, L.P. Heere, H.A. Keizer, J. Swinkels, F.T. Verstappen. Motorische grondeigenschappen. In: Training, prestatie en gezondheid in de spot, edited by F. T. Verstappen. Utrecht: Bunge, 1985.

12. Boyden T.W., R.W. Pamenter. Exercise and the thyroid. In: Exercise endocrinology, edited by K. Fotherby and S. B. Pal. Berlin: Walter de Gruyter, 1985.

13. Brooks G.A. Current concepts in lactate exchange. Med Sci Sports Exerc 23: 895-906. 1991.

14. Bruin G., H. Kuipers, H.A. Keizer, G.J. Vander Vusse. Adaptation and overtraining in horses subjected to increasing training loads. J Appl Physiol 76: 1908-1913 1994.

15. Buono M.J., J.E. Yeager, J.A. Hodgdon. Plasma adrenocorticotropin and cortisol responses to brief high- intensity exercise in humans. J Appl Physiol 61: 1337-1339. 1986.

16. Challis J.R.A., B. Crabtree, E.A. Newsholme. Hormonal regulation of the rate of the glycogen/glucose-1-phosphate cycle in skeletal muscle. Bioch J 163: 205-210 1987

17. Chapman R.F., J. Stray-Gundersen, B.D. Levine. Individual variation in response to altitude training. J Appl Physiol 85: 1448-1456. 1998.

18. Chatard J.C., I. Mujika, C. Guy, J.R. Lacour. Anaemia and iron deficiency in athletes. Practical recommendations for treatment. Sports Med 27: 229-240. 1999.

19. Chrousos G.P., P.W. Gold. The concepts of stress and stress system disorders. 
Overview of physical and behavioral homeostasis. Jama 267: 1244-1252. 1992.

20. Connett R.J., T.E. Gayeski, C.R. Honig. Lactate accumulation in fully aerobic, working, dog gracilis muscle. Am J Physiol 246: H120-128. 1984.

21. Convertino V.A. Blood volume: its adaptation to endurance training. Med Sci Sports Exerc 23: 1338-1348. 1991.

22. Costill D.L., M.G. Flynn, J.P. Kirwan, J.A. Houmard, J.B. Mitchell, R. Thomas, S.H. Park. Effects of repeated days of intensified training on muscle glycogen and swimming performance. Med Sci Sports Exerc 20: 249-254. 1988.

23. Cumming D.C., S.R. Wall, H.A. Quinney, A.N. Belcastro. Decrease in serum testosterone levels with maximal intensity swimming exercise in trained male and female swimmers. Endocr Res 13: 31-41 1987.

24. Dohm G.L., T.M. Louis. Changes in androstenedione, testosterone and protein metabolism as a result of exercise. Proc Soc Exp Biol Med 158: 622-625. 1978.

25. Dressendorfer R.H., C.E. Wade, E.A. Amsterdam. Development of of pseudoanemia in marathon runners during a 20-day road race. Jama 246: 1215 1218. 1981.

26. Dufaux B., A. Hoederath, I. Streitberger, W. Hollmann, G. Assmann. Serum ferritin, transferrin, haptoglobin, and iron in middle- and long- distance runners, elite rowers, and professional racing cyclists. Int J Sports Med 2: 43-46. 1981.

27. Ekblom B., A.N. Goldbarg, B. Gullbring. Response to exercise after blood loss and reinfusion. J Appl Physiol 33: 175-180. 1972.

28. Farrell P.A., M. Kjaer, F.W. Bach, H. Galbo. Beta-endorphin and adrenocorticotropin response to supramaximal treadmill exercise in trained and untrained males. Acta Physiol Scand 130: 619-625. 1987.

29. Finkelstein J.W., H.P. Roffwarg, R.M. Boyar, J. Kream, L. Hellman. Age-related change in the twenty-four-hour spontaneous secretion of growth hormone. J Clin Endocrinol Metab 35: 665-670. 1972.

30. Flynn M.G., F.X. Pizza, J.B. Boone, Jr., F.F. Andres, T.A. Michaud, J.R. RodriguezZayas. Indices of training stress during competitive running and swimming seasons. Int J Sports Med 15: 21-26. 1994.

31. Fry R.W., A.R. Morton, D. Keast. Periodisation and the prevention of overtraining. Can J Sport Sci 17: 241-248. 1992.

32. Fryburg D.A., E.J. Barrett. Growth hormone acutely stimulates skeletal muscle but not whole-body protein synthesis in humans. Metabolism 42: 1223-1227. 1993.

33. Fryburg D.A., R.A. Gelfand, E.J. Barrett. Growth hormone acutely stimulates forearm muscle protein synthesis in normal humans. Am J Physiol 260: E499-504. 1991.

34. Gladden L.B. Muscle as a consumer of lactate. Med Sci Sports Exerc 32: 764-771. 2000.

35. Gledhill N. The influence of altered blood volume and oxygen transport capacity on aerobic performance. Exerc Sport Sci Rev 13: 75-93 1985.

36. Green H.J., S. Carter, S. Grant, R. Tupling, G. Coates, M. Ali. Vascular volumes and hematology in male and female runners and cyclists. Eur J Appl Physiol Occup Physiol 79: 244-250. 1999.

37. Greenspan S.L., A. Klibanski, D. Schoenfeld, E.C. Ridgway. Pulsatile secretion of thyrotropin in man. J Clin Endocrinol Metab 63: 661-668. 1986.

38. Guglielmini C., I. Casoni, M. Patracchini, F. Manfredini, G. Grazzi, M. Ferrari, $\mathrm{F}$. Conconi. Reduction of $\mathrm{Hb}$ levels during the racing season in nonsideropenic 
professional cyclists. Int J Sports Med 10: 352-356. 1989.

39. Hackney A.C. Endurance training and testosterone levels. Sports Med 8: 117-127. 1989.

40. Hackney A.C., T. Gulledge. Thyroid hormone responses during an 8-hour period following aerobic and anaerobic exercise. Physiol Res 43: 1-5 1994.

41. Hackney A.C., R.J. Ness, A. Schrieber. Effects of endurance exercise on nocturnal hormone concentrations in males. Chronobiol Int 6: 341-346 1989.

42. Hackney A.C., W.E. Sinning, B.C. Bruot. Reproductive hormonal profiles of endurance-trained and untrained males. Med Sci Sports Exerc 20: 60-65. 1988.

43. Hickson R.C., J.M. Hagberg, A.A. Ehsani, J.O. Holloszy. Time course of the adaptive responses of aerobic power and heart rate to training. Med Sci Sports Exerc 13: 17-20 1981.

44. Hooper S.L., L.T. Mackinnon. Monitoring overtraining in athletes. Doctoral Dissertation.The University of Queensland, Department of Human Movement Studies, Brisbane, 1998

45. Hooper S.L., L.T. Mackinnon, A. Howard, R.D. Gordon, A.W. Bachmann. Markers for monitoring overtraining and recovery. Med Sci Sports Exerc 27: 106-112. 1995.

46. Jeukendrup A.E., M.K. Hesselink. Overtraining--what do lactate curves tell us? BrJ Sports Med 28: 239-240 1994.

47. Jeukendrup A.E., M.K. Hesselink, A.C. Snyder, H. Kuipers, H.A. Keizer. Physiological changes in male competitive cyclists after two weeks of intensified training. Int J Sports Med 13: 534-541. 1992.

48. Jiang B., R.R. Roy, C. Navarro, V.R. Edgerton. Absence of a growth hormone effect on rat soleus atrophy during a 4-day spaceflight. J Appl Physiol 74: 527-531. 1993.

49. Keast D., D. Arstein, W. Harper, R.W. Fry, A.R. Morton. Depression of plasma glutamine concentration after exercise stress and its possible influence on the immune system. Med J Aust 162: 15-18. 1995.

50. Keizer H.A., H. Kuipers, J. de Haan, E. Beckers, L. Habets. Multiple hormonal responses to physical exercise in eumenorrheic trained and untrained women. Int J Sports Med 8 Suppl 3: 139-150. 1987.

51. Keizer H.A., H. Kuipers, J. de Haan, G.M. Janssen, E. Beckers, L. Habets, G. van Kranenburg, P. Geurten. Effect of a 3-month endurance training program on metabolic and multiple hormonal responses to exercise. Int J Sports Med 8 Suppl 3: 154-160. 1987.

52. Keizer H.A., J. Poortman, G.S. Bunnik. Influence of physical exercise on sex-hormone metabolism. J Appl Physiol 48: 765-769. 1980.

53. Keizer H.A., A.D. Rogol. Physical exercise and menstrual cycle alterations. What are the mechanisms? Sports Med 10: 218-235. 1990.

54. Kern W., B. Perras, R. Wodick, H.L. Fehm, J. Born. Hormonal secretion during nighttime sleep indicating stress of daytime exercise. J Appl Physiol 79: 1461-1468 1995.

55. Kindermann W. Das Ubertraining-Ausdruck einer vegetativen Fehlsteuerung. Dtsch Z Sportmed 37: 138-145 1986.

56. Kjaer M., N.H. Secher, F.W. Bach, S. Sheikh, H. Galbo. Hormonal and metabolic responses to exercise in humans: effect of sensory nervous blockade. Am J Physiol 257: E95-101. 1989. 
57. Knobil E. The neuroendocrine control of ovulation. Hum Reprod 3: 469-472. 1988.

58. Kraemer W.J., S.J. Fleck, R. Callister, M. Shealy, G.A. Dudley, C.M. Maresh, L. Marchitelli, C. Cruthirds, T. Murray, J.E. Falkel. Training responses of plasma beta-endorphin, adrenocorticotropin, and cortisol. Med Sci Sports Exerc 21: 146153. 1989.

59. Kraemer W.J., S.J. Fleck, J.E. Dziados, E.A. Harman, L.J. Marchitelli, S.E. Gordon, R. Mello, P.N. Frykman, L.P. Koziris, N.T. Triplett. Changes in hormonal concentrations after different heavy-resistance exercise protocols in women. J Appl Physiol 75: 594-604 1993.

60. Kraicer J., M.S. Sheppard, J. Luke, B. Lussier, B.C. Moor, J.S. Cowan. Effect of withdrawal of somatostatin and growth hormone $(\mathrm{GH})$-releasing factor on $\mathrm{GH}$ release in vitro. Endocrinology 122: 1810-1815. 1988.

61. Kreider R., A. Fry, M.L. O'Tool. Overtraining in Sport. Champaign, USA: Human Kinetics, 1998.

62. Kuipers $\mathrm{H}$. Training and overtraining: an introduction. Med Sci Sports Exerc 30: 1137-11391998.

63. Kuipers H., H.A. Keizer. Overtraining in Elite Athletes, Review and directions for the Furture. Sports medicine 6: 79-92 1988.

64. Kuipers H., H.A. Keizer, G. Schep, A.R. Hoogeveen. Long-term intensive athletic training; few adverse effects on later physical health. Ned Tijdschr Geneeskd 145: 1101-1104. 2001.

65. Kuipers H., F.T.J. Verstappen, H.A. Keizer, P. Geurten, G. Kranenburg van. Variability of Aerobic Performance in the Laboratory and Its Physiologic Correlates. Sport Med 6: 197-201 1985.

66. Lebovitz H.E., K. Bryant, L.A. Frohman. Acute effects of corticotropin and related peptides on carbohydrate and lipid metabolism. Ann N Y Acad Sci 131: 274-287. 1965.

67. Lehmann M., P. Baumgartl, C. Wiesenack, A. Seidel, H. Baumann, S. Fischer, U. Spori, G. Gendrisch, R. Kaminski, J. Keul. Training-overtraining: influence of a defined increase in training volume vs training intensity on performance, catecholamines and some metabolic parameters in experienced middle- and long-distance runners. Eur J Appl Physiol Occup Physiol 64: 169-177 1992.

68. Lehmann M., S. Baur, N. Netzer, U. Gastmann. Monitoring high-intensity endurance training using neuromuscular excitability to recognize overtraining. Eur J Appl Physiol Occup Physiol 76: 187-191 1997.

69. Lehmann M., H.H. Dickhuth, G. Gendrisch, W. Lazar, M. Thum, R. Kaminski, J.F. Aramendi, E. Peterke, W. Wieland, J. Keul. Training-overtraining. A prospective, experimental study with experienced middle- and long-distance runners. Int J Sports Med 12: 444-452. 1991.

70. Lehmann M., C. Foster, J. Keul. Overtraining in endurance athletes: a brief review. Med Sci Sports Exerc 25: 854-862 1993.

71. Lehmann M., U. Gastmann, K.G. Petersen, N. Bachl, A. Seidel, A.N. Khalaf, S. Fischer, J. Keul. Training-overtraining: performance, and hormone levels, after a defined increase in training volume versus intensity in experienced middle- and long-distance runners. Br J Sports Med 26: 233-242. 1992.

72. Lehmann M., K. Knizia, U. Gastmann, K.G. Petersen, A.N. Khalaf, S. Bauer, L. Kerp, J. Keul. Influence of 6-week, 6 days per week, training on pituitary function in 
recreational athletes. Br J Sports Med 27: 186-192 1993.

73. Lehmann M., H. Wieland, U. Gastmann. Influence of an unaccustomed increase in training volume vs intensity on performance, hematological and blood-chemical parameters in distance runners. J Sports Med Phys Fitness 37: 110-116. 1997.

74. Loucks A.B., J.F. Mortola, L. Girton, S.S. Yen. Alterations in the hypothalamicpituitary-ovarian and the hypothalamic-pituitary-adrenal axes in athletic women. J Clin Endocrinol Metab 68: 402-411. 1989.

75. Luger A., P.A. Deuster, S.B. Kyle, W.T. Gallucci, L.C. Montgomery, P.W. Gold, D.L. Loriaux, G.P. Chrousos. Acute hypothalamic-pituitary-adrenal responses to the stress of treadmill exercise. Physiologic adaptations to physical training. N Engl J Med 316: 1309-1315. 1987.

76. McColl E.M., G.D. Wheeler, P. Gomes, Y. Bhambhani, D.C. Cumming. The effects of acute exercise on pulsatile $\mathrm{LH}$ release in high-mileage male runners. Clin Endocrinol (Oxf) 31: 617-621 1989.

77. McNair D.M. Profile of Mood States Manual. Educational and Industrial Testing Service. San Diego, 1971.

78. Menheere P.P.C.A. Sensitive estimations of glucoprotein hormones; clinical and scientific applications. Doctoral Dissertation.University of LImburg, Maastricht, the Netherlands, 1989

79. Moretti C., A. Fabbri, L. Gnessi, M. Cappa, A. Calzolari, F. Fraioli, A. Grossman, G.M. Besser. Naloxone inhibits exercise-induced release of PRL and GH in athletes. Clin Endocrinol (Oxf) 18: 135-138. 1983.

80. Morgan W.P. Affective beneficence of vigorous physical activity. Med Sci Sports Exerc 17: 94-100. 1985.

81. Morgan W.P., D.R. Brown, J.S. Raglin, P.J. O'Connor, K.A. Ellickson. Psychological monitoring of overtraining and staleness. Br J Sports Med 21: 107-114 1987.

82. Morgan W.P., D.L. Costill, M.G. Flynn, J.S. Raglin, P.J. O'Connor. Mood disturbance following increased training in swimmers. Med Sci Sports Exerc 20: 408-414 1988.

83. Morley J.E., G.L. Brammer, B. Sharp, T. Yamada, A. Yuwiler, J.M. Hershman. Neurotransmitter control of hypothalamic-pituitary-thyroid function in rats. Eur J Pharmacol 70: 263-271. 1981.

84. Naveri H., K. Kuoppasalmi, M. Harkonen. Metabolic and hormonal changes in moderate and intense long-term running exercises. Int J Sports Med 6: 276-281. 1985.

85. Newhouse I.J., D.B. Clement. Iron status in athletes. An update. Sports Med 5: 337-352 1988.

86. Nieman D.C. Is infection risk linked to exercise workload? Med Sci Sports Exerc 32: S406-411. 2000.

87. O'Connor P.J. Overtraining and Staleness. In: Physical activity \& mental Health, edited by W. P. Morgan. Bristol: Taylor \& Francis, 1997, p. 146-159.

88. O'Connor P.J., W.P. Morgan, J.S. Raglin. Psychobiologic effects of $3 \mathrm{~d}$ of increased training in female and male swimmers. Med Sci Sports Exerc 23: $1055-10611991$.

89. Orth D.N., W.J. Kovacs, C. Rowan Debold. The Adrenal cortex. In: Williams textbook of endocrinolgy, edited by J. D. Wilson and D. W. Foster. Philadelphia: W.B. Saunders, 1992.

90. Overton J.M., K.C. Kregel, G. Davis-Gorman, D.R. Seals, C.M. Tipton, L.A. Fisher. Effects of exercise training on responses to central injection of CRF and noise 
stress. Physiol Behav 49: 93-98. 1991.

91. Pascoe D.D., L.B. Gladden. Muscle glycogen resynthesis after short term, high intensity exercise and resistance exercise. Sports Med 21: 98-118. 1996.

92. Peters E.M., E.D. Bateman. Ultramarathon running and upper respiratory tract infections. An epidemiological survey. S Afr Med J 64: 582-584. 1983.

93. Petraglia F., C. Barletta, F. Facchinetti, F. Spinazzola, A. Monzani, D. Scavo, A.R. Genazzani. Response of circulating adrenocorticotropin, beta-endorphin, beta- lipotropin and cortisol to athletic competition. Acta Endocrinol (Copenh) 118: 332-336. 1988.

94. Prinz P.N., E.D. Weitzman, G.R. Cunningham, I. Karacan. Plasma growth hormone during sleep in young and aged men. J Gerontol 38: 519-524. 1983.

95. Quabbe H.J., E. Schilling, H. Helge. Pattern of growth hormone secretion during a 24-hour fast in normal adults. J Clin Endocrinol Metab 26: 1173-1177. 1966.

96. Raglin J.S., W.P. Morgan, A.E. Luchsinger. Mood and self-motivation in successful and unsuccessful female rowers. Med Sci Sports Exerc 22: 849-853. 1990.

97. Raglin J.S., W.P. Morgan, P.J. O'Connor. Changes in mood states during training in female and male college swimmers. Int J Sports Med 12: 585-589 1991.

98. Reichlin S. Neuroendocrinology. In: Williams textbook of endocrinology, edited by J. D. Wilson and D. W. Foster. Philadelphia: W.B. Saunders, 1992.

99. Rogol A.D., A. Weltman, J.Y. Weltman, R.L. Seip, D.B. Snead, S. Levine, E.M. Haskvitz, D.L. Thompson, R. Schurrer, E. Dowling, et al. Durability of the reproductive axis in eumenorrheic women during $1 \mathrm{yr}$ of endurance training. J Appl Physiol 72: 1571-1580. 1992.

100. Ronsen O., K. Holm, H. Staff, P.K. Opstad, B.K. Pedersen, R. Bahr. No effect of seasonal variation in training load on immuno-endocrine responses to acute exhaustive exercise. Scand J Med Sci Sports 11: 141-148. 2001.

101. Rowbottom D.G., D. Keast, C. Goodman, A.R. Morton. The haematological, biochemical and immunological profile of athletes suffering from the overtraining syndrome. Eur J Appl Physiol Occup Physiol 70: 502-509 1995.

102. Sapolsky R.M., L.C. Krey, B.S. McEwen. Stress down-regulates corticosterone receptors in a site-specific manner in the brain. Endocrinology 114: 287-292. 1984

103. Sapolsky R.M., L.C. Krey, B.S. McEwen. The neuroendocrinology of stress and aging: the glucocorticoid cascade hypothesis. Endocrinology Reviews 7: 284-301 1986.

104. Slag M.F., M. Ahmad, M.C. Gannon, F.Q. Nuttall. Meal stimulation of cortisol secretion: a protein induced effect. Metabolism 30: 1104-1108. 1981.

105. Smith J.A. Exercise, training and red blood cell turnover. Sports Med 19: 9-31. 1995.

106. Snyder A.C., C. Foster. Physiology and nutrition for skating. In: Perspectives in exercise and sports medicine, edited by D. R. Lamb, H. G. Knuttgen and

R. Murray. Carmel, Indiana: Cooper Publishing group, 1994, p. 181-219.

107. Snyder A.C., H. Kuipers, B. Cheng, R. Servais, E. Fransen. Overtraining following intensified training with normal muscle glycogen. Med Sci Sports Exerc 27: 1063-1070. 1995.

108. Suetta C., I.L. Kanstrup, N. Fogh-Andersen. Haematological status in elite longdistance runners: influence of body composition. Clin Physiol 16: 563-574. 1996

109. Szygula Z. Erythrocytic system under the influence of physical exercise and training. Sports Med 10: 181-197. 1990.

110. Takahashi Y., D.M. Kipnis, W.H. Daughaday. Growth hormone secretion during 
sleep. J Clin Invest 47: 2079-2090. 1968.

111. Tannenbaum G.S., N. Ling. The interrelationship of growth hormone (GH)releasing factor and somatostatin in generation of the ultradian rhythm of $\mathrm{GH}$ secretion. Endocrinology 115: 1952-1957. 1984.

112. Urhausen A., H. Gabriel, W. Kindermann. Blood hormones as markers of training stress and overtraining. Sports Med 20: 251-276 1995.

113. Urhausen A., W. Kindermann. Behaviour of testosterone, sex hormone binding globulin (SHBG), and cortisol before and after a triathlon competition. Int J Sports Med 8: 305-308 1987.

114. van Breda E., H.A. Keizer, P. Geurten, G. van Kranenburg, P.P. Menheere, H. Kuipers, J.F. Glatz. Modulation of glycogen metabolism of rat skeletal muscles by endurance training and testosterone treatment. Pflugers Arch 424: 294-300. 1993.

115. van Breda E., H.A. Keizer, M.M. Vork, D.A. Surtel, Y.F. de Jong, G.J. van der Vusse, J.F. Glatz. Modulation of fatty-acid-binding protein content of rat heart and skeletal muscle by endurance training and testosterone treatment. Pflugers Arch 421: 274-279 1992.

116. Vanhelder W.P., R.C. Goode, M.W. Radomski. Effect of anaerobic and aerobic exercise of equal duration and work expenditure on plasma growth hormone levels. Eur J Appl Physiol Occup Physiol 52: 255-257 1984.

117. Veale D.M. Psychological aspects of staleness and dependence on exercise. Int J Sports Med 12 Suppl 1: S19-22. 1991.

118. Veldhuis J.D., A. Iranmanesh, G. Lizarralde, M.L. Johnson. Amplitude modulation of a burstlike mode of cortisol secretion subserves the circadian glucocorticoid rhythm. Am J Physiol 257: E6-14. 1989.

119. Verde T., S. Thomas, R.J. Shephard. Potential markers of heavy training in highly trained distance runners. Br/ Sports Med 26: 167-175. 1992.

120. Vervoorn C., L.J. Vermulst, A.M. Boelens-Quist, H.P. Koppeschaar, W.B. Erich, J.H. Thijssen, W.R. de Vries. Seasonal changes in performance and free testosterone: cortisol ratio of elite female rowers. Eur J Appl Physiol Occup Physiol 64: 14-21 1992.

121. Viru A. The mechanism of training effects: a hypothesis. Int / Sports Med S: 219-227 1984.

122. Viru A. Plasma hormones and physical exercise. Int J Sports Med 13: 201-209 1992.

123. Wasserman K., M.B. McLlroy. Detecting the threshold of anearobic metabolism in cardiac patients during exercise. Am J Cardiol 14: 844-852 1964.

124. Watanabe T., A. Morimoto, Y. Sakata, N. Tan, K. Morimoto, N. Murakami. Running training attenuates the ACTH responses in rats to swimming and cage-switch stress. J Appl Physiol 73: 2452-2456. 1992.

125. Wittert G.A., J.H. Livesey, E.A. Espiner, R.A. Donald. Adaptation of the hypothalamopituitary adrenal axis to chronic exercise stress in humans. Med Sci Sports Exerc 28: 1015-1019 1996. 

CHAPTER 2

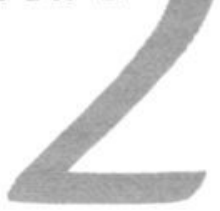

Physiological characteristics of elite Olympic distance triathletes

This Chapter has been submitted as:

Physiological characteristics of elite Olympic distance triathletes

G.J.W.M. Rietjens', H. Kuipers', L. Delahaye' ${ }^{2}$ and H.A. Keizer'

' Department of Movement Sciences, Maastricht University, Maastricht, The Netherlands

2 Netherlands Triathlon Federation (NTB), Nieuwegein, The Netherlands

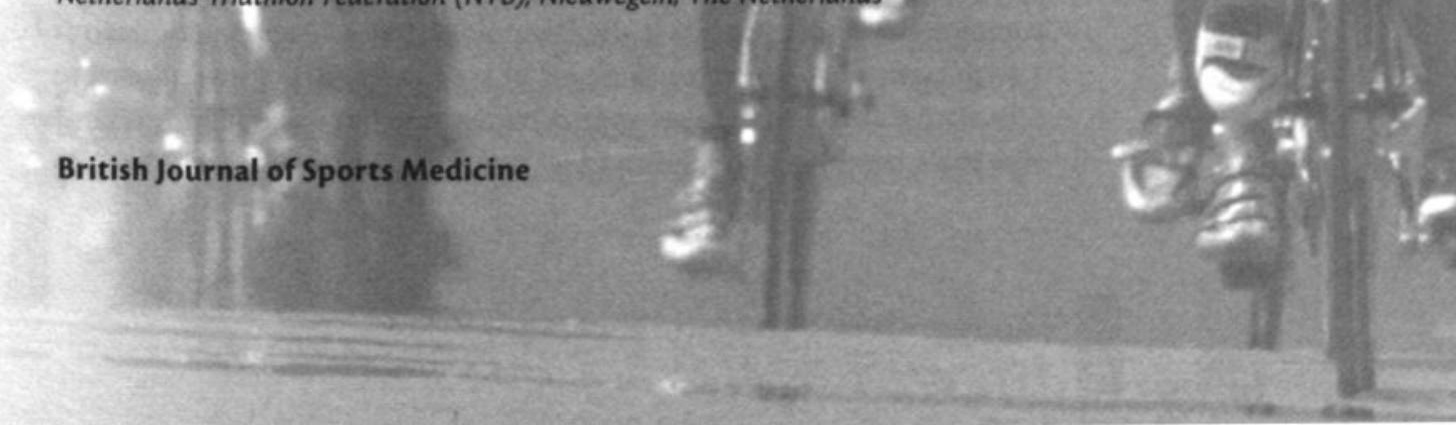




\section{Abstract}

Purpose: The aim of this study was to describe the physiological characteristics and possible changes within elite Olympic distance triathletes throughout the year.

Methods: In fifteen (nine male, six female) elite Olympic distance triathletes body height, weight and percentage of fat were measured. Furthermore, (sub) maximal oxygen uptake $\left(\mathrm{VO}_{2}\right)$, maximal work load (Wmax and Vmax), heart rate (HR) and lactate (L) were tested on a cycle ergometer and treadmill. In nine athletes these measurements were repeated 4 times throughout one year in order to investigate changes during this period of training and races.

Results: At the onset of the study mean body height was $184.7 \pm 5.4 \mathrm{~cm}$ and $170.8 \pm 10.1 \mathrm{~cm}$ for men and women, respectively. Body mass was $73.9 \pm 5.5 \mathrm{~kg}$ for men and $62.5 \pm 9.4 \mathrm{~kg}$ for women. The percentage of body fat was $9.6 \pm 1.7 \%$ in men and $20.0 \pm 4.2 \%$ in women. Maximal oxygen uptake on a cycle ergometer was $70.5 \pm 5.9 \mathrm{ml} \cdot \mathrm{kg}^{-1} \cdot \mathrm{min}^{-1}$ in men and $57.7 \pm 7.2 \mathrm{ml}$. $\mathrm{kg}^{-1} \cdot \mathrm{min}^{-1}$ in woman and on the treadmill $70.1 \pm 6.1 \mathrm{ml} \cdot \mathrm{kg}^{-1} \cdot \mathrm{min}^{-1}$ in men and $60.1 \pm 7.8 \mathrm{ml} \cdot \mathrm{kg}^{-1} \cdot \mathrm{min}^{-1}$ in women. In addition Maximal power output was $412 \pm 35$ Watt $(5.6 \pm 0.3 \mathrm{Watt} / \mathrm{kg})$ and $282 \pm 13 \mathrm{Watt}(5.2 \pm 0.7 \mathrm{Watt} / \mathrm{kg})$ in men and women, respectively. Maximal speed on the treadmill was $19.4 \pm 0.6 \mathrm{~km} / \mathrm{h}$ and $16.7 \pm 1.0 \mathrm{~km} / \mathrm{h}$, for men and women, respectively. Besides a significant reduction in sub maximal lactate values $(P<0.05)$ non of these values changed significantly during the training year.

Conclusion: Elite triathletes have still lower maximal oxygen uptake values compared to runners or cyclists. No changes in physiological performance level and related parameters could be observed throughout the year, despite considerable changes in training load.

\section{Introduction}

Triathlon is one of the fastest growing sports in the last decade. It includes three disciplines: swimming, cycling and running. The classic form (also known as IronMan triathlon) consists of $3.8 \mathrm{~km}$ of swimming, $180 \mathrm{~km}$ of cycling and $42.2 \mathrm{~km}$ of running, and competition time takes eight to eleven hours. Next to the classic form various other disciplines have been combined (ea. ice skating, cross country skiing, mountain biking) and different distances have been introduced in triathlon. Nowadays the most common and most popular is the Olympic distance triathlon consisting of $1.5 \mathrm{~km}$ swimming, $40 \mathrm{~km}$ cycling and $10 \mathrm{~km}$ running. The time needed to complete this triathlon takes approximately one hour and fifty minutes for elite male triathletes and around two hours for elite female triathletes. In 
the Sydney Olympic 2000 games, the Olympic distance triathlon made a successful Olympic début. Despite the short history of the triathlon sport, many scientific articles have been published and have described the physiological characteristics of triathletes $(4,8,11,16,19,21)$. However, most of these studies described the profile of triathletes competing in longer distance disciplines and most often the subjects were well trained, but were not elite athletes. Data on elite Olympic distance triathletes are scarce. As far as we know only one study has been reported, however, in this study only female athletes were involved (11). Therefore, the first objective of this study was to describe the physiological profile of both male and female elite Olympic distance triathletes.

Furthermore, almost all previous studies were cross sectional and described results of a single test. To our knowledge, only one study on seasonal changes has been reported in recreational triathletes (9). Therefore the second aim of this study was to determine if, and if so, how physiological and performance characteristics in elite triathletes change throughout the season.

\section{Material and methods}

\section{Subjects}

Six female (mean age $25.7 \pm 3.1$ years) and nine male (mean age $25.8 \pm 5.8$ years) elite Olympic distance $(O D)$ triathletes who were in full preparation for the oncoming Olympic 2000 games participated in this study, which was approved by the Medical Ethical Committee of the Maastricht University. One year later out of this group 4 female and 5 male athletes were selected for the Olympic team. These athletes were followed during the year in preparation of the Olympics 2000.

\section{General procedures}

As part of a total support program, all members of the Dutch national OD triathlon team underwent a cycle- and running exercise test just before the start of the 1998 race season (cross sectional data). From these athletes a selected group participated in a year round program of testing one year later (longitudinal data). Testing (test 1 ) began during the offseason. The second and third test was conducted half way and at the end of the training period. The last test session (test 4 ) was executed half way the race season. For every test session all subjects were tested within one week. All subjects were asked to refrain from intense exercise 24 hours preceding the test. For each test, the subjects reported at the laboratory between 8.00 and 9.00 A.M. For all subjects the cycle- and treadmill test took place at the same day always starting with the cycling test. After a 
break of at least two hours the running test was conducted. All tests took place under similar environmental conditions $\left(22 \pm 1.2^{\circ} \mathrm{C}, 50 \pm 12 \%\right.$ humidity). Before and during every test session, the following variables were measured: body weight (BW), percentage of body fat, (sub) maximal oxygen uptake $\left(\mathrm{VO}_{2}\right)$, maximal work load ( $\mathrm{Wmax}$ and $\mathrm{Vmax}$ ), heart rate (HR) and blood lactate (L) on both the bicycle test and the running test. During the bicycle test (Lode Excalibur Sport, Lode, Groningen The Netherlands, which was calibrated at rpm values between 70 and 100 $\mathrm{rpm}$ ) and the running test on a motor driven treadmill (Medifit, Maarn, The Netherlands), respiratory data were collected continuously by using a metabolic system (Oxycon B, E. Jaeger $\mathrm{GmbH}$, Höchberg, Germany).

The peak $\mathrm{VO}_{2}$ value obtained during a test was taken as $\mathrm{VO}_{2}$ max. Along with these measurements the heart rates were recorded at $30 \mathrm{sec}$ intervals (Polar Sporttester, Kempele, Finland). The maximal workload (Wmax) was defined as the last completed work load (W) plus the fraction of time spent in the final work rate multiplied by workload of the last completed step (10). From an indwelling catheter in an antecubital vein, blood samples for lactate were taken at rest and during the last 30 seconds of every step. The last blood sample was collected at exhaustion.

To get information of the training work of the subjects, subjects who were involved in the longitudinal part of the study were asked to record their daily training time spent on cycling and running.

\section{Measurements}

\section{Anthropometric parameters}

Body height, body weight and percentage body fat were measured. Percentage body fat was calculated using the skin fold method, according to Durnin and Womersley (6). To minimize errors all measurements were done in duplicate and by the same investigator.

\section{Maximal workload protocols \\ Cross sectional study}

Cycling. The test started with a 5 min warm up period ( $150 \mathrm{~W}$ for males and $100 \mathrm{~W}$ for females) where after the workload was increased every 5 minutes by 75 watt till a heart rate of 165 beats per min was reached. Then the workload was increased by $25 \mathrm{~W}$ every $5 \mathrm{~min}$ until a volitional exhaustion occurred.

Running. After a warm up period of 5 minutes $12 \mathrm{~km} / \mathrm{h}^{-1}$ and $10 \mathrm{~km} / \mathrm{h}^{-1}$ for men and women, respectively, the speed was increased by $2 \mathrm{~km} / \mathrm{h}^{-1}$ every 5 minutes until a heart rate of 165 beats per min was reached, where after the running speed was increased every $5 \mathrm{~min}$ by $1 \mathrm{~km} / \mathrm{h}^{-1}$ until exhaustion. 


\section{Longitudinal study}

Cycling. This cycle test consisted of three stages; an incremental stage towards a steady state level and a second incremental stage until exhaustion. The initial workload of the maximal workload test was set at 1 Watt per $\mathrm{kg}$ body weight (W/kg BW) for the female subjects and at $1.5 \mathrm{~W} / \mathrm{kg} \mathrm{BW}$ for the male subjects, were after increased by $1 \mathrm{~W} / \mathrm{kg} \mathrm{BW}$ every three minutes until a workload of 4 or $4.5 \mathrm{~W} / \mathrm{kg}$ BW was reached for females and males, respectively. At this level, the workload was maintained for twelve minutes. This point was chosen since it is assumed that this exercise intensity approximates the intensity during an actual competition. After the twelve minutes lasting step, the workload was increased again every three minutes by $0.5 \mathrm{~W} / \mathrm{kg}$ BW (for both the females and males) until a volitional exhaustion occurred.

Running. Similar to the cycling protocol, the running protocol was divided into three stages. The initial speed for the female subjects was $11.5 \mathrm{~km} / \mathrm{h}^{-1}$, ( $13.5 \mathrm{~km} / \mathrm{h}^{-1}$ for males) with an increment of $2 \mathrm{~km} / \mathrm{h}^{-1}$ every $3 \mathrm{~min}$. The twelve-minute step was at a speed of $16.5 \mathrm{~km} / \mathrm{h}^{-1}\left(18.5 \mathrm{~km} / \mathrm{h}^{-1}\right.$ for males). Thereafter the speed was increased again every three minutes by $1 \mathrm{~km} / \mathrm{h}^{-1}$ until volitional exhaustion. The incline of the treadmill was kept constant at $1 \%$.

\section{Statistical analyzes}

Data in the cross sectional study are expressed as mean \pm standard deviation. Data in the longitudinal study are expressed as adjusted mean \pm standard deviation. Statistical analyzes for changes throughout the season were accomplished using a three-way analysis of variance. Where appropriate, a Tukey post hoc test was performed to determine the location of significant difference between the means. $P<0.05$ was considered significant.

\section{Results}

\section{Cross sectional data}

The cross sectional data are presented in table 1. Mean body height was $184.7 \pm 5.4 \mathrm{~cm}$ for men and $170.8 \pm 10.1 \mathrm{~cm}$ for the women. Body mass was $73.9 \pm 5.5 \mathrm{~kg}$ and $62.5 \pm 9.4 \mathrm{~kg}$, respectively for the male and female athletes. The percentage of body fat was $9.6 \pm 1.7 \%$ and $20.0 \pm 4.2 \%$ for male and female athletes, respectively. The mean group aerobic power $\left(\mathrm{VO}_{2} \mathrm{max}\right)$ obtained during the incremental cycle test was $65 \pm 8.9 \mathrm{ml} \cdot \mathrm{kg}^{-1} \cdot \mathrm{min}^{-1}$ $\left(70.5 \pm 5.9 \mathrm{ml} \cdot \mathrm{kg}^{-1} \cdot \mathrm{min}^{-1}\right.$ in men and $57.7 \pm 7.2 \mathrm{ml} \cdot \mathrm{kg}^{-1} \cdot \mathrm{min}^{-1}$ in women). Maximal power output during this incremental test was $359.8 \pm 71.1$ Watt $(5.2 \pm 0.7 \mathrm{watt} / \mathrm{kg})$. The males reached an average of $412 \pm 35 \mathrm{Watt}$ 
$(5.6 \pm 0.3 \mathrm{Watt} / \mathrm{kg})$ while the female athletes had an average power output of $282 \pm 13$ Watt $(5.2 \pm 0.7$ Watt $/ \mathrm{kg})$.

Accordingly, group mean $\mathrm{VO}_{2}$ max values during treadmill running were $66.1 \pm 8.3 \mathrm{ml} \cdot \mathrm{kg}^{-1} \cdot \mathrm{min}^{-1}\left(70.1 \pm 6.1 \mathrm{ml} \cdot \mathrm{kg}^{-1} \cdot \mathrm{min}^{-1}\right.$ in men and $60.1 \pm 7.8 \mathrm{ml} \cdot \mathrm{kg}^{-1}$ - $\min ^{-1}$ in women) at an average maximal speed of $18.3 \pm 1.5 \mathrm{~km} / \mathrm{h}^{-1}$ (19.4 $\pm 0.6 \mathrm{~km} / \mathrm{h}^{-1}$ and $16.7 \pm 1.0 \mathrm{~km} / \mathrm{h}^{-1}$, for men and women, respectively).

\section{TABLE 1}

Mean $( \pm S D)$ values of anthropometric and physiological characteristics of elite male and female Olympic distance triathletes

\begin{tabular}{|c|c|c|c|c|c|c|c|c|}
\hline & \multirow[b]{2}{*}{$\begin{array}{l}\text { Length } \\
(\mathrm{cm})\end{array}$} & \multirow[b]{2}{*}{$\begin{array}{l}\text { Weight } \\
(\mathrm{kg})\end{array}$} & \multirow[b]{2}{*}{$\begin{array}{l}\text { Body fat } \\
(\%)\end{array}$} & \multicolumn{3}{|c|}{ CYCLING } & \multicolumn{2}{|c|}{ RUNNING } \\
\hline & & & & $\begin{array}{c}\mathrm{VO}_{2} \max \\
\left(\mathrm{ml} \cdot \mathrm{kg}^{-1} \cdot \min ^{-1}\right)\end{array}$ & $\begin{array}{c}W \max \\
(W)\end{array}$ & $\begin{array}{c}W / k g B W \\
(W)\end{array}$ & 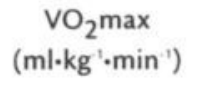 & $\begin{array}{c}V \max \\
\left(\mathrm{km} \cdot \mathrm{h}^{-1}\right)\end{array}$ \\
\hline \multicolumn{9}{|l|}{ male } \\
\hline $\mathbb{B}_{1}$ & 181 & 75.1 & 9 & 69.8 & 425 & 5.7 & 75.5 & 19.0 \\
\hline$x_{2}$ & 183 & 77 & 6.8 & 78.9 & 403 & 5.9 & 76.8 & 19.4 \\
\hline$x_{3}$ & 182 & 72 & 7.5 & 72.9 & 403 & 6.0 & 68.5 & 19.0 \\
\hline 84 & 187 & 84.3 & 9.8 & 63.9 & 405 & 5.4 & 64.9 & 20.0 \\
\hline 85 & 185 & 68.5 & 11.5 & 68.2 & 370 & 5.1 & 69.0 & 20.3 \\
\hline 6 & 183 & 67 & 9.1 & 78.4 & 458 & 5.9 & 80.6 & 19.6 \\
\hline 7 & 197 & 77 & 11.3 & 73.1 & 465 & 5.5 & 64.4 & 18.4 \\
\hline 8 & 186 & 75.7 & 11.5 & 67.4 & 415 & 5.4 & 68.5 & 19.4 \\
\hline 9 & 178 & 68.4 & 10 & 62.0 & 360 & 5.3 & 63.1 & 19.2 \\
\hline mean & 184.7 & 73.9 & 9.6 & 70.5 & 411.6 & 5.6 & 70.1 & 19.4 \\
\hline sd & 5.4 & 5.5 & 1.7 & 5.9 & 35.0 & 0.3 & 6.1 & 0.6 \\
\hline \multicolumn{9}{|l|}{ female } \\
\hline $\mathbb{B}_{1}$ & 167 & 58 & 15.5 & 63.4 & 283 & 4.9 & 68.8 & 17.4 \\
\hline 82 & 170 & 57.4 & 17.4 & 60.1 & 275 & 4.8 & 58.1 & 16.0 \\
\hline $8_{3}$ & 170 & 60.5 & 22.6 & 59.5 & 306 & 5.1 & 65.9 & 18.2 \\
\hline 84 & 157 & 52 & 17.4 & 65.1 & 270 & 5.2 & 65.4 & 16.9 \\
\hline 5 & 173 & 69.4 & 26.8 & 51.3 & 275 & 4.0 & 52.6 & 16.1 \\
\hline 6 & 188 & 77.7 & 20.3 & 46.7 & 284 & 3.7 & 49.8 & 15.6 \\
\hline mean & 170.8 & 62.5 & 20 & 57.7 & 282.2 & 4.6 & 60.1 & 16.7 \\
\hline sd & 10.1 & 9.4 & 4.2 & 7.2 & 12.8 & 0.6 & 7.8 & 1.0 \\
\hline \multicolumn{9}{|l|}{ overall } \\
\hline mean & 179.1 & 69.3 & 13.8 & 65.4 & 359.8 & 5.2 & 66.1 & 18.3 \\
\hline sd & 10.1 & 9.1 & 6.0 & 8.9 & 71.1 & 0.7 & 8.3 & 1.5 \\
\hline
\end{tabular}

athletes who were also involved in the longitudinal study 


\section{Longitudinal data}

The average weekly training load is presented in table 2 . The training load (cycling and running) increased significantly $(P<0.05)$ from the start of the pre season ( $722 \pm 52 \mathrm{~min} / \mathrm{wk}$ ) towards the end of the pre season ( $961 \pm 84$ $\mathrm{min} / \mathrm{wk}$ ), followed be a slight non significant decrease during the race season $(823 \pm 96 \mathrm{~min} / \mathrm{wk})$.

\section{TABLE 2}

Training volume between the several test

$$
\text { TEST 1-2* }
$$

Training volume $\left(\min \cdot w^{-1}\right)$

Cycle

$$
\begin{aligned}
& 488 \pm 35 \\
& 284 \pm 31 \\
& 772 \pm 52
\end{aligned}
$$$$
591 \pm 59
$$

Run

$370 \pm 41$ $316 \pm 42$

Overall

$961 \pm 84 \#$

$823 \pm 96$

$\because$ training period between test 1 and test 2 , between test 2 and test 3 and between test 3 and test 4 respectively, throughout the year.

Values are mean \pm SD. \# significant difference between training period 1-2 and 2-3

Mean body weight and percentage of body fat showed the highest values during the off-season and the lowest values during the race season but these differences were not significant. In both running and cycling sub maximal values of HR or oxygen uptake did not change over the year (table 3). Sub maximal lactate during the cycling test was significantly lower in test 3 and 4 compared to test 1 . Despite a trend towards lowering the sub maximal lactate level throughout the season no significant changes were found in the running tests. Maximal heart rate, oxygen uptake and lactate did not change over time in both cycling and running. Maximal workload obtained during the cycle tests and maximal running speed did not change significantly either over the year. 


\section{TABLE 3}

Sub maximal and maximal values ( $n=15$, nine men and six women) obtained during the off season (test 1), the training season (test 2 and 3 ) and during the race season (test 4).

Values are adjusted means \pm SD. \# season effect between test 1 and $4(P<0.05)$

\begin{tabular}{|c|c|c|c|c|}
\hline & Test 1 & Test 2 & Test 3 & Test 4 \\
\hline Weight (kg) & $65.3 \pm 8.9$ & $64.6 \pm 9.6$ & $64.1 \pm 10.3$ & $64 \pm 9.4$ \\
\hline Body Fat (\%) & $12.6 \pm 5.1$ & $12.8 \pm 5.1$ & $12.7 \pm 5.0$ & $11.3 \pm 4.4$ \\
\hline \multicolumn{5}{|c|}{ Heart rate (bpm) } \\
\hline \multicolumn{5}{|l|}{ Sub-maximal } \\
\hline Cycle & $171 \pm 8$ & $169 \pm 11$ & $167 \pm 8$ & $168 \pm 11$ \\
\hline Run & $178 \pm 4$ & $178 \pm 5$ & $177 \pm 6$ & $176 \pm 5$ \\
\hline \multicolumn{5}{|l|}{ Maximal } \\
\hline Cycle & $179 \pm 8$ & $179 \pm 3$ & $181 \pm 7$ & $177 \pm 10$ \\
\hline Run & $183 \pm 6$ & $183 \pm 7$ & $185 \pm 5$ & $184 \pm 7$ \\
\hline \multicolumn{5}{|c|}{$\mathrm{VO}_{2} \max \left(\mathrm{ml} \cdot \mathrm{kg}^{-1} \cdot \mathrm{min}^{-1}\right)$} \\
\hline \multicolumn{5}{|c|}{ Sub-maximal } \\
\hline Cycle & $60.2 \pm 5.3$ & $60.5 \pm 5.7$ & $59.7 \pm 5.3$ & $60.8 \pm 4.9$ \\
\hline Run & $59.9 \pm 4.9$ & $59.5 \pm 5.1$ & $60.9 \pm 4.4$ & $61.8 \pm 3.4$ \\
\hline \multicolumn{5}{|l|}{ Maximal } \\
\hline Cycle & $63.6 \pm 5.8$ & $63.9 \pm 6.5$ & $65.1 \pm 5.6$ & $64.9 \pm 6.4$ \\
\hline Run & $62.9 \pm 5.6$ & $63.9 \pm 5.6$ & $67.1 \pm 3.4$ & $66.3 \pm 4.3$ \\
\hline \multicolumn{5}{|l|}{ Max workload } \\
\hline Cycle (W) & $315 \pm 68$ & $312 \pm 67$ & $331 \pm 70$ & $328 \pm 73$ \\
\hline $\operatorname{Run}\left(k \cdot h^{-1}\right)$ & $18.4 \pm 1.3$ & $18.5 \pm 1.3$ & $18.9 \pm 1.6$ & $19.1 \pm 1.4$ \\
\hline \multicolumn{5}{|c|}{ Lactate $\left(\mathrm{mmol} \cdot \mathrm{I}^{\prime}\right)$} \\
\hline \multicolumn{5}{|l|}{ Sub-maximal } \\
\hline Cycle & $10.3 \pm 0.5$ & $8.5 \pm 0.6$ & $7.6 \pm 0.7$ & $7.1 \pm 0.6 \#$ \\
\hline Run & $6.1 \pm 0.4$ & $5.0 \pm 0.4$ & $4.3 \pm 0.5$ & $4.9 \pm 0.4$ \\
\hline \multicolumn{5}{|l|}{ Maximal } \\
\hline Cycle & $13.0 \pm 3.7$ & $11.4 \pm 1.9$ & $12.1 \pm 1.9$ & $10.3 \pm 3.1$ \\
\hline Run & $8.3 \pm 2.3$ & $9.6 \pm 2.9$ & $9.0 \pm 1.2$ & $8.2 \pm 1.7$ \\
\hline
\end{tabular}




\section{Discussion}

To compete successfully in a triathlon, the athletes have to perform in three different sports (swimming, cycling, running), and in each of these disciplines different physiological characteristics are of advantage. For instance in swimming a high percentage of body fat may improve buoyancy. A tall body height is also an advantage during swimming since longer limbs allow a longer stroke, which is in general more economical $(2,26)$. On the other hand, a high body mass (gravity) and high body length (surface area) are of disadvantage during cycling and running (7, 14). In general it seems that triathletes are shorter as elite swimmers (26), have the same height as elite cyclists $(12,14)$ but tend to be taller than elite runners (18). This holds for both genders. In the present study, the average percentage of body fat $(9.6 \%)$ in males is similar to other studies in long distance triathletes $(1,3,9,16)$. The female athletes in this study showed a percentage of body fat $(20.0 \%)$ which was somewhat higher than found in other studies $(5,11,22)$. This difference can (partly) be explained by a high percentage of body fat $(27 \%)$ in one of the female athletes. In line with the higher average percentage of body fat, the female athletes in this study also have a higher mean body weight compared to previous studies $(5,22)$. Compared to these studies on Olympic- and long distance triathletes, both the male and female athletes in the present study have on average a larger body height, which is comparable to elite swimmers $(11,24)$. An explanation for this can be that most of the subjects involved in this study had chosen swimming as specialisation before entering triathlons.

The maximal aerobic power for cycling and running found in the present study is in line with most other studies. The small differences between all these studies can be explained by the different test protocols used $(1,3,5$, $8,9,11,16,25)$. One of the differences in incremental test protocols may be the time per stage. In a recent study Kuipers et al (2001, submitted) demonstrated that peak $\mathrm{VO}_{2}$ in an incremental test on a cycle ergometer was significantly higher (3.6\%) with increments every minute, compared to increments every 3 and 5 minutes.

A remarkable observation made in the present study was that for any testing session no differences in $\mathrm{VO}_{2}$ max were found between the cycling and running test whereas in other studies in triathletes $\mathrm{VO}_{2}$ max in cycling is $2-6 \%$ lower than $\mathrm{VO}_{2}$ max during running $(5,9,11,16)$. The difference in $\mathrm{VO}_{2}$ max values as found in other studies in triathletes is difficult to explain but may be attributed to the fact that in most other studies the subjects were not elite athletes. It has been shown that athletes who are trained in a particular sport may have similar or higher $\mathrm{VO}_{2} \mathrm{max}$ when tested in the specific sport, compared with treadmill tests $(23,27)$. 
When comparing the $\mathrm{VO}_{2}$ max values of the present study with measurements in elite runners or cyclists, it can be observed that the $\mathrm{VO}_{2} \max$ values of these elite triathletes are still somewhat lower than in elite runners and cyclists. This is also true for the maximal power output obtained during an incremental bicycle test, which is in line with other investigations in triathletes $(13,17)$. Although these differences cannot be explained easily, it is possible that the lack of specialization in one sport may underlay these differences. The weekly average training load (hours/wk) measured in the present study is equal as reported in other studies on elite OD triathletes $(11,15)$ and shows a clear pattern of an increased training volume towards the race season. Despite this significant increase in training volume, no changes were found in maximal $\mathrm{VO}_{2}$, lactate, heart rate and power output throughout the season. These findings are in line with a longitudinal study of Kohrt and co workers in long distance triathletes, in which they also failed to find any significant changes in maximal values during a whole triathlon season (9).

The absence of any fluctuation in maximal values throughout a whole year can be explained by the fact that all subjects in the present study maintained their training during the off-season. This explanation is supported by a study of Rietjens and co-workers, who showed that well-trained cyclist could maintain their high fitness level during 21-days of reduced training (20). The only significant change during the season was the lowering of the sub maximal lactate at a given power output which is in line with the findings of Kohrt et al who found also a shift towards lower sub maximal lactate (lactate threshold at a higher $\% \mathrm{VO}_{2} \max$ ) during the season (9). Therefore, from the results from the present study and the study of Kohrt and co workers (9), one can conclude that the sub maximal lactate is a good indicator of the training status of the individual athlete, taken his own (previous) values as control.

In conclusion, our results confirm and extend previous studies in triathletes.

Furthermore, we found that elite triathletes do not reach the maximal oxygen uptake and maximal performance level found in elite cyclists or runners, even as we take into account the longer duration of the exercise test used in the present study. Probably as a result of continuing training (even during the off-season), elite triathletes do not show fluctuations in the classical indicators of physiological performance except for a lower sub maximal lactate value during the peak season. 


\section{Acknowledgement}

This research was supported by a grant from the Netherlands Olympic Committee (NOC*NSF). The authors express their gratitude to Dennis van Hamont, Ingrid van de Port, Dotje Hennekes, Michel Blijlevens and Kirsten Frick for their assistance during all the testing. 


\section{References}

1. Bunc V., J. Heller, J. Horcic, J. Novotny. Physiological profile of best Czech male and female young triathletes. J Sports Med Phys Fitness 36: 265-270 1996.

2. Craig A.B., Jr., P.L. Skehan, J.A. Pawelczyk, W.L. Boomer. Velocity, stroke rate, and distance per stroke during elite swimming competition. Med Sci Sports Exerc 17: 625-634. 1985.

3. De Vito G., M. Bernardi, E. Sproiero, F. Figura. Decrease in endurance performace during Olympic triathlon. Int J Sports Med 16: 24-28 1995.

4. Deitrick R.W. Physiological responses of typical versus heavy weight triathletes to treadmill and bicycle exercise. J Sports Med Phys Fitness 31: 367-375 1991.

5. Dengel D.R., M.G. Flynn, D.L. Costill, J.P. Kirwan. Determinants of success during triathlon competition. Res Q Exerc Sport 60: 234-238 1989.

6. Durnin J.V.G.A., J. Womersley. Body fat assessed from total body density and its estimation from skinfold thickness: measurement on 481 men and women aged from 16 to 72 year. Brit. J. Nutr. 32: 77-97 1974.

7. Gregor R.J., F. Conconi, J.P. Broker. Biomechanics of road cycling. In: Road Cycling (1st ed.), edited by R. J. Gregor and F. Conconi. Oxford: Blackwell Science, 2000, p. 18-39.

8. Holly R.G., R.J. Barnard, M. Rosenthal, E. Applegate, N. Pritikin. Triathlete characterization and response to prolonged strenuous competition.

Med Sci Sports Exerc 18: 123-127 1986.

9. Kohrt W.M., J.S. O'Connor, J.S. Skinner. Longitudinal assessment of responses by triathletes to swimming, cycling, and running. Med Sci Sports Exerc 21: 569-575 1989.

10. Kuipers H., F.T.J. Verstappen, H.A. Keizer, P. Geurten, G. Kranenburg van. Variability of Aerobic Performance in the Laboratory and Its Physiologic Correlates. Sport Med 6: 197-201 1985.

11. Laurenson N.M., K.Y. Fulcher, P. Korkia. Physiological characteristics of elite and club level female triathletes during running. Int J Sports Med 14: 455-459 1993.

12. Lucia A., J. Hoyos, J.L. Chicharro. Physiology of professional road cycling. Sports Med 31: 325-3372001.

13. Lucia A., H. Joyos, J.L. Chicharro. Physiological response to professional road cycling: climbers vs. time trialists. Int J Sports Med 21: 505-512. 2000.

14. Neumann. Cycling. In: Endurance in Sport (1st edn ed.), edited by R. J. Shephard and P. O. Astrand. Oxford: Blackwell Science, 1992, p. 582-596.

15. O'Toole M.L., P.S. Douglas, W.D. Hiller. Applied physiology of a triathlon. Sports Med 8: 201-225 1989.

16. O'Toole M.L., D.B. Hiller, L.O. Crosby, P.S. Douglas. The ultraendurance triathlete: a physiological profile. Med Sci Sports Exerc 19: 45-50 1987.

17. Padilla S., I. Mujika, G. Cuesta, J.J. Goiriena. Level ground and uphill cycling ability in professional road cycling. Med Sci Sports Exerc 31: 878-885. 1999.

18. Pollack M.L. Characteristics of elite class distance runners. Annals of the New York Acadamy of Sciences 3001: 278-282 1977.

19. Rensburg van J., A.J. Kielblock, d.L.A. van. Physiologic and biochemical changes during a triathlon competition. Int J Sports Med 7: 30-35 1986. 
20. Rietjens G.J.W.M., H. Kuipers, W.H.M. Saris, H.A. Keizer. A reduction in training volume and intensity during 21-days does not impair performance in cyclists. BrJ Sports Med 35: 431-434 2001.

21. Roalstad M.S. Physiologic testing of the ultraendurance triathlete. Med Sci Sports Exerc: 1989.

22. Schneider D.A., J. Pollack. Ventilatory threshold and maximal oxygen uptake during cycling and running in female triathletes. Int J Sports Med 12: 379-383. 1991.

23. Shephard R.J. Maximal Oxygen Intake. In: Endurance in Sport (second edition ed.), edited by R. J. Shephard and P. O. Astrand. Oxford: Blackwell Science, 2000, p. 301-327.

24. Sleivert G.G. The triathlon. In: Endurance in sport (second edition ed.), edited by R. J. Shephard and P. O. Astrand. Oxford: Blackwell science, 2000, p. 872-887.

25. Sleivert G.G., H.A. Wenger. Physiological predictors of short-course triathlon performance. Med Sci Sports Exerc 25: 871-876. 1993.

26. Tittel K., H. Wutsherk. Anatomical and anthropometric fundamentals of endurance. In: Endurance in sport (1st edn ed.), edited by R. J. Shephard and P. O. Astrand. Oxford: Blackwell Science, 1992.

27. Verstappen F.T., R.M. Huppertz, L.H. Snoeckx. Effect of training specificity on maximal treadmill and bicycle ergometer exercise. Int J Sports Med 3: 43-46. 1982. 



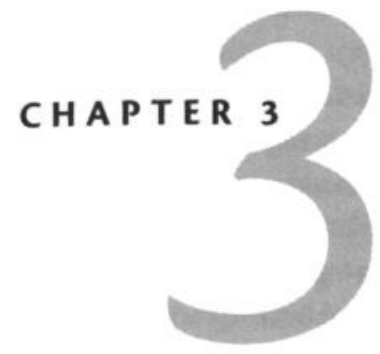

\section{Red blood cell profile of elite Olympic distance triathletes; A three-year follow-up}

This Chapter has been accepted for publication as:

Red blood cell profile of elite Olympic distance triathletes; A three-year follow-up

G.J.W.M. Rietjens', H. Kuipers', F.Hartgens ${ }^{2}$ and H.A. Keizer'

' Nutrition and Toxicology Research Institute Maastricht (NUTRIM)

Department of Movement Sciences, Maastricht University, Maastricht, The Netherlands

2 Medical Doctor, Dutch Triathlon Federation, Nieuwegein, The Netherlands

International Journal of Sports Medicine 


\section{Abstract}

Purpose: The purpose of this study was to monitor mean and individual changes in hematological variables during long-term endurance training, detraining and altitude training in elite Olympic distance triathletes.

Methods: Over a period of three years, a total of 102 blood samples were collected in eleven ( 7 male and 4 female) elite Olympic distance triathletes (mean \pm SD; age $=26.4 \pm 5.1 \mathrm{yr} ; \mathrm{VO}_{2} \max =67.9 \pm 6.6 \mathrm{ml} \cdot \mathrm{kg}^{-1} \cdot \mathrm{min}^{-1}$ ) for determination of hemoglobin ( $\mathrm{Hb})$, hematocrit $(\mathrm{Hct})$, red blood cell count (RBC), Mean corpuscular hemoglobin ( $\mathrm{MCH})$, Mean corpuscular hemoglobin content (MCHC), Mean corpuscular volume (MCV) and plasma ferritin. The data were pooled and divided into three periods; off-season, training season and race season. Blood samples obtained before and after altitude training were analyzed separately.

Results: Of all measured variables only RBC showed a significant decrease $(P<0.05)$ during the race season compared to the training season. Hematological values below the lower limit of the normal range were found in $46 \%$ of the athletes during the off-season. This percentage increased from $55 \%$ during the training season to $72 \%$ of the athletes during the race season. Hemoglobin and ferritin values were most frequently below the normal range. There was a weak correlation between $\mathrm{Hb}$ levels and $\mathrm{VO}_{2}$ max during maximal cycling $(\mathrm{r}=0.084)$ and running $(r=0.137)$ tests. Unlike training at $1500 \mathrm{~m}$ and $1850 \mathrm{~m}$, training at an altitude of $2600 \mathrm{~m}$ for three weeks showed significant increases in $\mathrm{Hb}$ (+ $10 \% ; \mathrm{P}<0.05), \mathrm{Hct}(+11 \% ; \mathrm{P}<0.05)$ and MCV $(+5 \% ; \mathrm{P}<0.05)$. Conclusion: Long-term endurance training does not profoundly alter hematological status. However, regular screening of hematological variables is desirable as many athletes have values near or below the lower limit of the normal range. The data obtained from altitude training suggest that a minimum altitude ( $>2000 \mathrm{~m}$ ) is necessary to alter hematological status.

Key words: triathletes, endurance training, altitude training, red blood cells

\section{Introduction}

Several studies have reported that endurance athletes, as compared to non-trained subjects, may show hematological abnormalities and decreased hemoglobin levels, near to or below the lower limit of the normal range $(5,27)$. Despite several studies in this area, there are limited data on the long-term changes in hematological variables associated with 
long-term endurance training. In addition, description of hematological changes throughout the entire season and in conjunction with altitude training are scarce. Especially in the light of a possible manipulated increase in red cell mass by exogenous erythropoietin, data on normal values and normal variation throughout the season are valuable.

Therefore, the aim of this study was to monitor changes in hematological variables during long-term endurance training in elite Olympic distance triathletes. In addition, possible changes associated with altitude training are reported. Not only average patterns, but also individual differences in hematological response are reported.

\section{Material and methods}

\section{Subjects}

Four female and seven male elite Olympic distance triathletes who were in full preparation for the Olympic 2000 games participated in the study, which was approved by the Medical Ethical Committee of the Maastricht University. Subjects' physical characteristics at commencement of the study are presented in Table 1. None of the subjects was vegetarian and none was blood donor. All athletes used iron supplements during altitude training. Throughout the training and race season only athletes with ferritin levels below $30 \mu \mathrm{g} / \mathrm{l}$ were supplemented orally with iron ( $25 \mathrm{mgr} /$ day, Ortica 25 complex) until the hematological status was in the normal range again.

\section{TABLE 1}

Physical characteristics at commencement of the study

\begin{tabular}{|lll|}
\hline \multicolumn{1}{|c|}{ VARIABLE } & FEMALES & MALES \\
\hline Age $(\mathrm{yr})$ & $26.0 \pm 2.2$ & $26.6 \pm 6.4$ \\
Body Weight $(\mathrm{kg})$ & $57.0 \pm 3.6$ & $72.0 \pm 4.0$ \\
Body Fat $(\%)$ & $18.2 \pm 3.1$ & $10.0 \pm 2.4$ \\
$\operatorname{VO} 2$ max $\left(\mathrm{ml}^{\prime} \cdot \mathrm{kg}^{-1} \cdot \mathrm{min}^{-1}\right)$ & $62.0 \pm 2.7$ & $71.2 \pm 5.8$ \\
\hline
\end{tabular}




\section{Blood sampling}

Over a period of three years, blood samples were collected every time the athletes reported to the laboratory for a performance test and a general medical check up. The collected samples were pooled into three groups; 1) samples taken during off-season when athletes were involved in relatively moderate programs, 2 ) the training season and 3 ) during the race season.

The training season started in January and ended mid March, the race season ended late October. During the training season, altitude training camps at various altitudes $(1500 \mathrm{~m}-1850 \mathrm{~m}-2600 \mathrm{~m})$ were part of the training schedule. Every altitude training lasted three weeks. On the average, $9 \pm 2$ (mean \pm SD) samples were collected per athlete during the three-year survey. All blood sampling was done between 08.00 and 10.00 A.M. The subjects were asked to refrain from training the day before. Before blood sampling the athletes rested on a bed for at least ten minutes. Blood samples $(7 \mathrm{ml})$ were taken from the athlete in supine position from an antecubital vein using the Vacuette( system and collected in tubes containing EDTA or no anticoagulant, the latter for harvesting of serum.

Hematological analysis consisting of a seven-parameter profile was performed using a Sysmex SE-9000 analyzer (Long Grove, USA). Serum ferritin was measured by immunoradiometric assay (Tandem-R ferritin, Hybritech Europe, Liege, Belgium).

The following hematological variables were determined; Hemoglobin $(\mathrm{Hb})$, hematocrit (Hct), red blood cell count (RBC), Mean corpuscular hemoglobin ( $\mathrm{MCH})$, Mean corpuscular hemoglobin content (MCHC), Mean corpuscular volume (MCV) and plasma ferritin.

\section{Statistical analyzes}

Data are expressed as mean \pm standard deviation. Statistical analyzes for changes throughout the season were assessed using a three-way analysis of variance. Where appropriate, a Tukey post hoc test wás performed to determine the location of significant difference between the means. Linear regression was used to determine the Pearson product moment correlation between hemoglobin and maximal oxygen uptake $\left(\mathrm{VO}_{2} \max \mathrm{ml} \cdot \mathrm{kg}^{-1} \cdot \mathrm{min}^{-1}\right)$. In order to determine differences between pre- and post altitude measurements a nonparametric ranking test of Wilcoxon was used. A P-value of $<0.05$ was considered significant. 


\section{Results}

The results are shown in Tables 2 and 3 and Figures 1 and 2.

When the same season during the three years was compared no significant differences in overall mean values were found. In every season, all mean group variables were within the (low) normal ranges (Table 2).

\section{TABLE 2}

Mean values $( \pm S D)$ for the examined variables in the different genders and total group over the three periods. ( $M=$ male and $F=$ female)

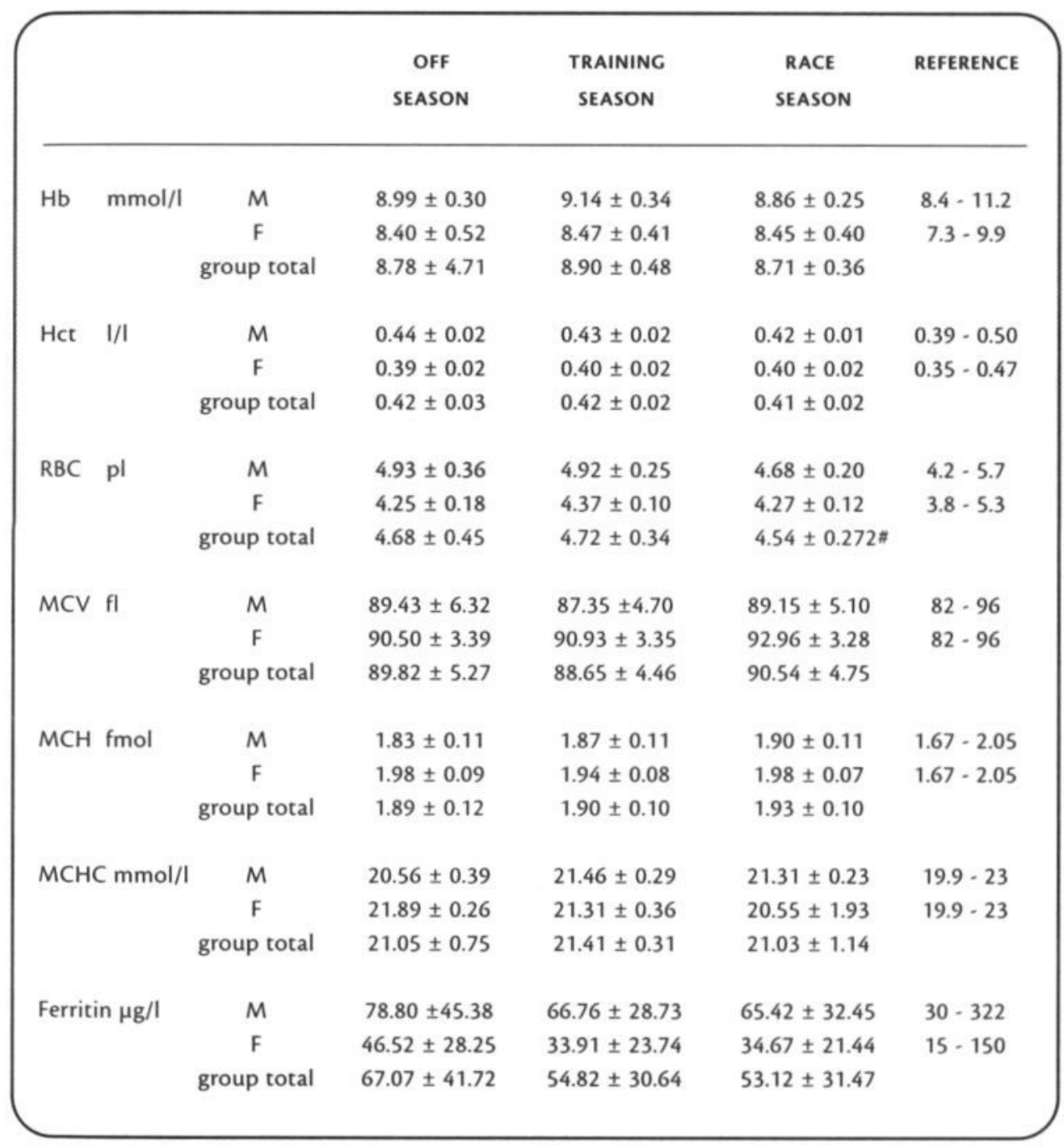

Values are mean $\pm S D$, \# Significantly different from training season, $\mathrm{P}<0.05$ 
Mean RBC reduced significantly $(P<0.05)$ between the training and race season. Although not significant, mean $\mathrm{Hb}$ and $\mathrm{Hct}$ values tended to be highest during the training season. The lowest values for $\mathrm{Hb}, \mathrm{Hct}, \mathrm{RBC}$ and ferritin were found in the female athletes.

A

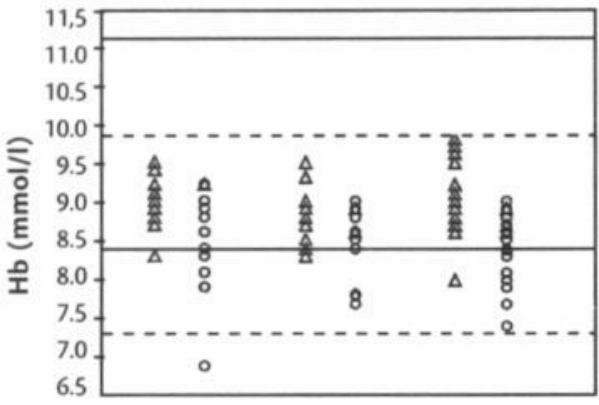

C

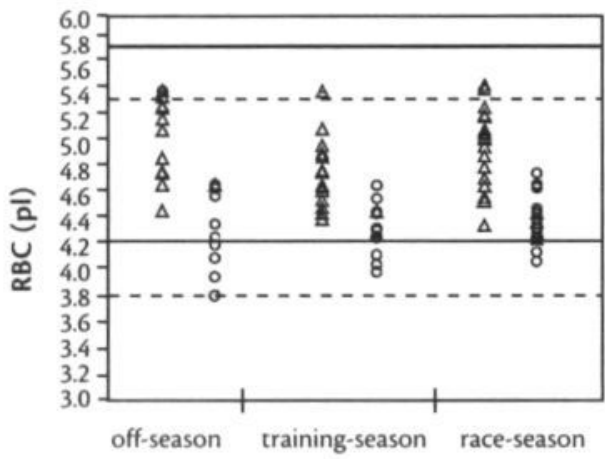

B

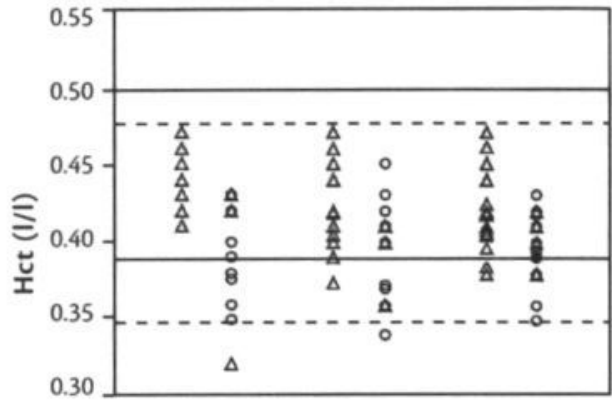

D

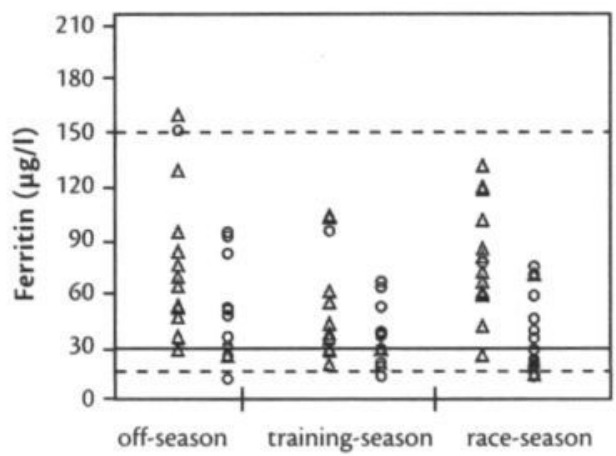

FIGURE 1

Evolution of hemoglobin (panel A), Hematocrit (panel B), Red blood cell Count (panel C), and ferritin (panel D) over the three seasons (102 samples) for both the male $(\Delta)$ and the female $(O)$ athletes. Solid line is the normal range for males, dashed line is normal range for females.

Individually, several athletes (both males and females) had values below the normal range as defined by the clinical laboratory (Medische Laboratoria dr. Stein \& Collegae, Maastricht, The Netherlands).

Off-season, five $(\approx 46 \%$ ) out of eleven athletes (one male and four female athletes) had one or more of the hematological variables below the normal range; two athletes had $\mathrm{Hb}$ values below the normal range, one athlete a reduced $\mathrm{Hct}$, three athletes had ferritin levels below $30 \mu \mathrm{g} / \mathrm{l}$, one had a decreased $\mathrm{MCH}$ level and one had reduced MCV values. The number of athletes with one ore more of the hematological variables 
under the normal range increased during the training season to six $(\approx 55 \%)$ athletes, with a further increase during the race season to eight $(\approx 72 \%)$ athletes. This was mainly caused by decreased $\mathrm{Hct}$ and $\mathrm{Hb}$ values. Whereas during the training season, two athletes (one male and one female athlete) had $\mathrm{Hb}$ and $\mathrm{Hct}$ values below the normal range, during the training-and race season the number of athletes with reduced $\mathrm{Hb}$ or $\mathrm{Hct}$ values increased to three (one male and two female athletes) and six (two males and four female athletes), respectively. There was a slight difference between female and male athletes concerning $\mathrm{Hb}, \mathrm{Hct}$ and $\mathrm{RBC}$. For these variables the male athletes showed a pronounced decline with their lowest values during the race season whereas the female athletes were more constant over the seasons.

Figure 2 shows the relationship between $\mathrm{Hb}$ and $\mathrm{VO}_{2}$ max. A weak correlation was found between hemoglobin levels and $\mathrm{VO}_{2}$ max obtained during both incremental cycle tests $(r=0.084)$ and treadmill running tests $(r=0.137)$.

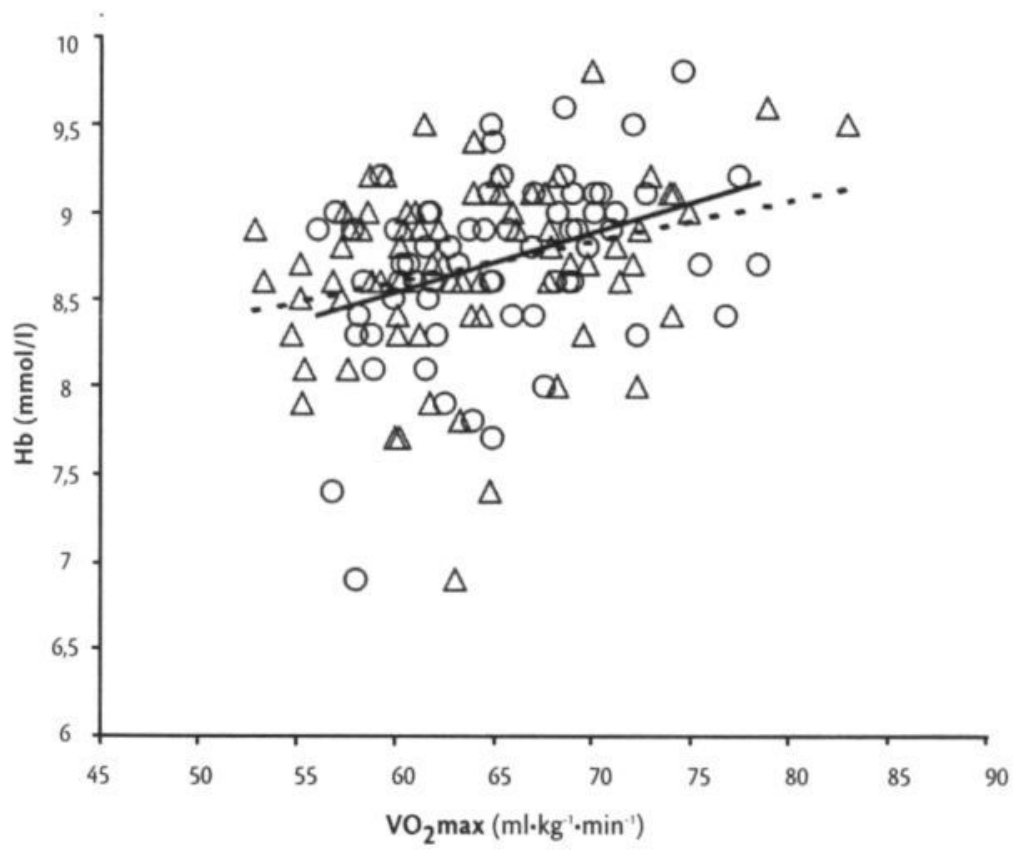

\section{FIGURE 2}

Relationship between $\mathrm{Hb}$ concentrations and VO2max obtained during cycling $(\Delta$, solid regression line) and running $(O$, dashed regression line in seven male and four female triathletes. 


\section{TABLE 3}

MCV, Hemoglobin and Hematocrit response to the three different altitudes.

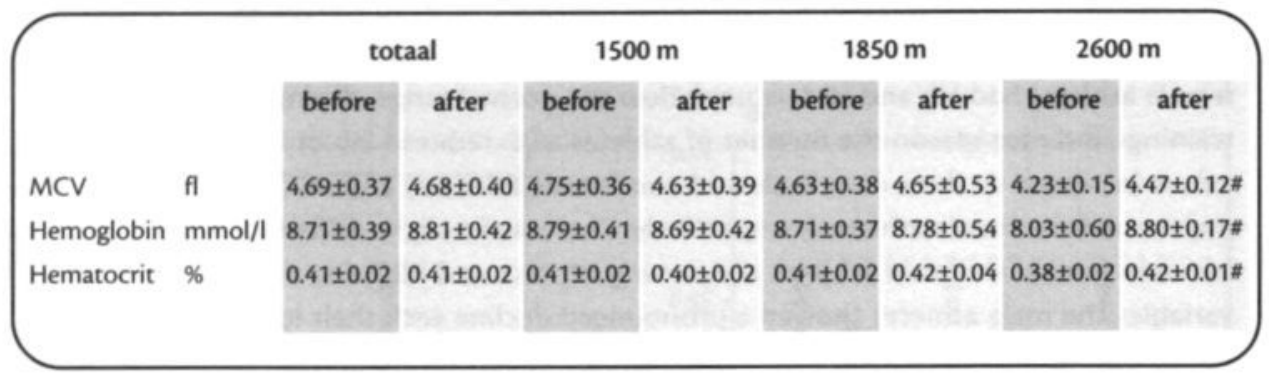

Values are mean \pm SD, \# Significantly different fro Pre-altitude, $P<0.005$.

Mean ferritin concentrations showed a decline throughout the year; highest values were observed during the off-season and lowest values were found during the race season $(67.1 \pm 41.7 \mu \mathrm{g} / \mathrm{l}, 54.8 \pm 30.6 \mu \mathrm{g} / \mathrm{l}$ and $50.1 \pm 31.5 \mu \mathrm{g} / \mathrm{l}$ for the off-, pre- and race season, respectively). Mean ferritin levels of the female athletes were during all seasons lower compared to their male counterparts.

The number of athletes $(n=3 ; 27 \%)$ with a reduced ferritin level in the offseason increased during the training season to four athletes (36\%). This remained unchanged during the race season. The lowest values $(11 \mu \mathrm{g} / \mathrm{l}$ and $13 \mu \mathrm{g} / \mathrm{l})$ were found during the race season in two female athletes.

For the pooled data of all altitude training camps there was no significant change in any of the variables measured. However, analyzing the altitude stages separately (table 3), a significant increase in $\mathrm{Hb}(10 \% ; \mathrm{P}<0.05)$, Hct (11\%; P < 0.05) and MCV (5\%; P < 0.05) was found after altitude training at $2600 \mathrm{~m}(\mathrm{n}=5)$. No significant changes were found after altitude training camps at lower altitudes $(1500 \mathrm{~m}(n=6)$ and $1850 \mathrm{~m}(n=7))$.

\section{Discussion}

The main purpose of our study was to monitor hematological variables during three consecutive years of training, in male and female elite Olympic distance triathletes, in order to establish normal variations throughout a year, including altitude training.

The data show that only mean RBC decreased significantly after the onset of the race season during the three-year survey. Throughout the year, mean $\mathrm{Hb}$ values were near the lower end of the normal range and were lowest during the race season. Our results are in line with other 
investigations in endurance athletes, reporting that most hematological variables do not change significantly during the different training phases of a year (33). The observed reduction in RBC during the race season is also in line with observations in elite runners, cyclists, swimmers and triathletes $(12,22,24)$. The low $R B C, H b$ and $\mathrm{Hct}$ values in athletes are sometimes considered to reflect sports anemia. Several hypotheses have been postulated to explain sport anemia in endurance athletes: gastrointestinal and/or urinary tract bleeding $(13,21,25)$, an increased iron loss or a decreased iron uptake $(13,26,31)$, reduced erythropoiesis (14), and plasma volume expansion (11). A true anemia is associated with iron deficiency and low iron stores reflected in low ferritin concentrations. Despite a decline in ferritin levels, they remained within the normal range, suggesting that there was no true iron deficiency. However, the decline in ferritin levels may indicate that iron loss was incompletely matched by iron intake during periods of severe exercise. Decreases in plasma ferritin levels have also been shown to occur during periods of intense training by various investigators $(3,5,15,19,20,23)$. The results of our study and those from other investigators suggest that iron supplementation during periods of severe training may be helpful. This is supported by the results of Gugliemini et al (12) and Dufaux et al (8), who demonstrated that ferritin levels did not decrease when iron supplementation was given during periods of heavy training.

In the present study a difference in some hematological variables between males and females was found. This confirms earlier studies in both trained and untrained subjects and these gender differences are generally considered as a normal difference $(2,15,34)$. A remarkable finding in the present study was that the female athletes showed a rather stable pattern of $\mathrm{Hb}, \mathrm{Hct}$ and $\mathrm{RBC}$ between the seasons, while the male athletes showed more variability with a decrease in these variables towards the race season. In an earlier prospective study with both male and female athletes, a similar pattern was found for $\mathrm{Hb}$ and RBC (15). The data of the present study do not allow drawing conclusions as to the underlying mechanism of this larger variability in males.

Since the hemoglobin content of the blood is a determining factor for its oxygen transport capacity, we also studied the relationship between hemoglobin content and $\mathrm{VO}_{2}$ max. It was found that the correlation between $\mathrm{Hb}$ and $\mathrm{VO}_{2}$ max measured on a cycle ergo meter $(r=0.084)$ and measured on a treadmill $(r=0.14, P>0.05)$ was weak. These results indicate that differences in $\mathrm{Hb}$ are not necessarily matched by parallel changes in $\mathrm{VO}_{2} \max$, and emphasize that the results of Kanstrup and Ekblom and Gore et al, who reported a strong relationship between the $\mathrm{Hb}$ content and $\mathrm{VO}_{2}$ max should be regarded with caution $(10,16)$. With regard to individual patterns, it was found that throughout the year 
several athletes had $\mathrm{Hb}, \mathrm{Hct}$ and ferritin values below the normal range, with the lowest values in the female athletes. In endurance athletes, a compromised hematological status as a result of intensive training has been reported earlier $(5-7,12,24,27)$ and the percentages of athletes with $\mathrm{Hb}$ values below the normal range $(\approx 9 \%, \approx 9 \%$ and $\approx 18 \%$ in the off-season, training and race season, respectively) as found in this study are in line with the findings of Weight and co-workers (in distance runners $11.7 \%$ ) (32). Again these results emphasize the importance of monitoring hematological status and possible iron supplementation.

In the present study we also investigated the effect of altitude training on hematological status. In this study, hematological variables from five altitude camps with different altitudes ( $1500 \mathrm{~m}, 1850 \mathrm{~m}$, and $2600 \mathrm{~m}$ ) showed a significant increase in MCV, $\mathrm{Hb}$ and $\mathrm{Hct}$ only after an altitude exposure of $2600 \mathrm{~m}$. Therefore, the results of the present study do not fully support previous research, in which exposure to lower altitudes was found to induce increases in MCV, $\mathrm{Hb}$ and $\operatorname{Hct}(1,9,17,30)$. Based on reports of Stray-Gundersen and co workers, who found a high number of non-responders in MCV after altitude training among athletes with iron deficiency and low ferritin levels, it could be argued that in the present study the iron stores may have been below optimal $(28,29)$.

However, since three subjects involved in altitude training at 2600 meter showed an increase in $\mathrm{MCV}, \mathrm{Hb}$ and $\mathrm{Hct}$, despite relatively low ferritin concentrations ( $36 \pm 12 \mu \mathrm{g} / \mathrm{l}$ ), the low ferritin concentrations that occurred in spite of iron supplementation are probably not the only reason for a lack of $\mathrm{Hb}$ response. The finding that only altitude training at $2600 \mathrm{~m}$ caused a significant increase in MCV, $\mathrm{Hb}$ and $\mathrm{Hct}$, supports the hypothesis that there exists a 'threshold altitude' beyond which sufficient stimulation of erythropoiesis occurs $(4,18)$.

In conclusion, the results of the present study demonstrate that male and female elite triathletes can maintain a normal hematological status throughout a year with regular monitoring and supplementation based on declines in ferritin levels. Nevertheless, even despite iron supplementation in athletes with ferritin concentrations below $30 \mu \mathrm{g} / \mathrm{l}$ and during altitude training, most of the variables were at the lower end of the normal range, or even below $(\mathrm{Hb}, \mathrm{Hct})$. Altitude training did not significantly increase the hematological status unless training was executed at an altitude of $2600 \mathrm{~m}$. 


\section{Acknowledgement}

This research was supported by a grant from the Netherlands Olympic Committee (NOC*NSF) The authors express their gratitude to Dr. D. Sewell for his assistance. 


\section{References}

1. Berglund B., H. Safstrom. Psychological monitoring and modulation of training load of world-class canoeists. Med Sci Sports Exerc 26: 1036-1040 1994.

2. Boyadjiev N., Z. Taralov. Red blood cell variables in highly trained pubescent athletes: a comparative analysis. Br J Sports Med 34: 200-204. 2000.

3. Candau R., T. Busso, J.R. Lacour. Effects of training on iron status in cross-country skiers. Eur J Appl Physiol Occup Physiol 64: 497-502 1992.

4. Chapman R.F., J. Stray-Gundersen, B.D. Levine. Individual variation in response to altitude training. J Appl Physiol 85: 1448-1456. 1998.

5. Clement D.B., R.C. Asmundson, C.W. Medhurst. Hemoglobin values: comparative survey of the 1976 Canadian Olympic team. Can Med Assoc J 117: 614-616. 1977.

6. de Wijn J.F., J.L. de Jongste, W. Mosterd, D. Willebrand. Haemoglobin, packed cell volume, serum iron and iron binding capacity of selected athletes during training. J Sports Med Phys Fitness 11: 42-51. 1971.

7. de Wijn J.F., J.L. de Jongste, W. Mosterd, D. Willebrand. Hemoglobin, packed cell volume, serum iron and iron binding capacity of selected athletes during training. Nutr Metab 13: 129-139 1971.

8. Dufaux B., A. Hoederath, I. Streitberger, W. Hollmann, G. Assmann. Serum ferritin, transferrin, haptoglobin, and iron in middle- and long- distance runners, elite rowers, and professional racing cyclists. Int J Sports Med 2: 43-46. 1981.

9. Eckardt K.U., U. Boutellier, A. Kurtz, M. Schopen, E.A. Koller, C. Bauer. Rate of erythropoietin formation in humans in response to acute hypobaric hypoxia. J Appl Physiol 66: 1785-1788. 1989.

10. Gore C.J., A.G. Hahn, C.M. Burge, R.D. Telford. VO2max and haemoglobin mass of trained athletes during high intensity training. Int J Sports Med 18: 477-482. 1997.

11. Green H.J., J.R. Sutton, G. Coates, M. Ali, S. Jones. Response of red cell and plasma volume to prolonged training in humans. J Appl Physiol 70: 1810-1815. 1991.

12. Guglielmini C., I. Casoni, M. Patracchini, F. Manfredini, G. Grazzi, M. Ferrari, F. Conconi. Reduction of $\mathrm{Hb}$ levels during the racing season in nonsideropenic professional cyclists. Int J Sports Med 10: 352-356. 1989.

13. Haymes E.M., J.J. Lamanca. Iron loss in runners during exercise. Implications and recommendations. Sports Med 7: 277-285. 1989.

14. Hunding A., R. Jordal, P.E. Paulev. Runner's anemia and iron deficiency. Acta Med Scand 209: 315-318 1981.

15. Janssen G.M.E. Marathon Running, Functional changes in male and female volunteers during training and contest. Doctoral Dissertation. Maastricht University, Maastricht, 1988

16. Kanstrup I.L., B. Ekblom. Blood volume and hemoglobin concentration as determinants of maximal aerobic power. Med Sci Sports Exerc 16: 256-262. 1984.

17. Klausen T., T. Mohr, U. Ghisler, O.J. Nielsen. Maximal oxygen uptake and erythropoietic responses after training at moderate altitude. Eur J Appl Physiol Occup Physiol 62: 376-379 1991.

18. Levine B.D., J. Stray-Gundersen. A practical approach to altitude training: where to live and train for optimal performance enhancement. Int J Sports Med 13 Suppl 1: S209-212. 1992. 
19. Magnusson B., E. Bjorn-Rassmussen, L. Hallberg, L. Rossander. Iron absorption in relation to iron status. Model proposed to express results to food iron absorption measurements. Scand J Haematol 27: 201-208. 1981.

20. Magnusson B., L. Hallberg, L. Rossander, B. Swolin. Iron metabolism and "sports anemia". I. A study of several iron parameters in elite runners with differences in iron status. Acta Med Scand 216: 149-155 1984.

21. Nachtigall D., P. Nielsen, R. Fischer, R. Engelhardt, E.E. Gabbe. Iron deficiency in distance runners. A reinvestigation using $\mathrm{Fe}$ - labelling and non-invasive liver iron quantification. Int / Sports Med 17: 473-479. 1996.

22. O'Toole M.L., W.D. Hiller, M.S. Roalstad, P.S. Douglas. Hemolysis during triathlon races: its relation to race distance. Med Sci Sports Exerc 20: 272-275. 1988.

23. Roberts D., D.J. Smith. Training at moderate altitude: iron status of elite male swimmers. J Lab Clin Med 120: 387-391. 1992.

24. Schumacher Y.O., D. Grathwohl, J.M. Barturen, M. Wollenweber, L. Heinrich, A. Schmid, G. Huber, J. Keul. Haemoglobin, haematocrit and red blood cell indices in elite cyclists. Are the control values for blood testing valid? Int / Sports Med 21: 380-385. 2000.

25. Siegel A.J., C.H. Hennekens, H.S. Solomon, B. Van Boeckel. Exercise-related hematuria. Findings in a group of marathon runners. Jama 241: 391-392. 1979.

26. Snyder A.C., L.L. Dvorak, J.B. Roepke. Influence of dietary iron source on measures of iron status among female runners. Med Sci Sports Exerc 21: 7-10. 1989.

27. Stewart G.A., J.E. Steel, A.H. Toyne, M.J. Stewart. Observations on the haematology and the iron and protein intake of Australian olympic athletes. Med J Aust 2: 1339-1343. 1972.

28. Stray-Gundersen J., B.D. Levine. Failure of red cell volume to increase with altitude exposure in iron deficient runners (abstract). Med Sci Sports Exerc 29: S136 1992.

29. Stray-Gundersen J., N. Mordecai, B.D. Levine. $O 2$ transport response to altitude training in runners (abstract). Med Sci Sports Exerc 27: S202 1995.

30. Terrados N., E. Jansson, C. Sylven, L. Kaijser. Is hypoxia a stimulus for synthesis of oxidative enzymes and myoglobin? J Appl Physiol 68: 2369-2372. 1990.

31. Weight L.M., P. Jacobs, T.D. Noakes. Dietary iron deficiency and sports anaemia. Br J Nutr 6 8: 253-260. 1992.

32. Weight L.M., M. Klein, T.D. Noakes, P. Jacobs. 'Sports anemia'--a real or apparent phenomenon in endurance-trained athletes? Int J Sports Med 13: 344-347. 1992.

33. White J.A., G. Quinn, M. Al-Dawalibi, J. Mulhall. Seasonal changes in cyclists' performance. Part I. The British Olympic road race squad. Br / Sports Med 16: 4-12. 1982.

34. Williams W.J., D.A. Nelson, M.W. Morris. Examination of the blood. In: Hematology, edited by W. J. Williams, E. Beutler, A. J. Erslev and M. A. Lichtman. New York: McGraw-Hill, 1990. 



\section{Abstract}

The aim of the present study was to evaluate the behaviour of the hormones (ACTH, cortisol, Growth Hormone, IGF-1) and the binding protein IGF-BP3 of the hypothalamic-pituitary axis (HPA) throughout a competitive season in elite triathletes, and to investigate whether changes in training load parallel changes in plasma hormone values. Eight (four male and 4 female) top-level triathletes (age $27 \pm 5$ yr, body height $175 \pm$ $10 \mathrm{~cm}$, body weight $\left.65 \pm 9 \mathrm{~kg}, \mathrm{VO}_{2} \max 66 \pm 6 \mathrm{ml} \cdot \mathrm{kg}^{-1} \cdot \mathrm{min}^{-1}\right)$ were tested four times throughout the year. Exercise tests were performed on a cycle ergometer. Blood samples were collected at rest, during sub maximal exercise and immediately after maximal exercise on the bicycle ergometer. There were no changes found in $\mathrm{Wmax}_{\max } \mathrm{VO}_{2} \max$. or lactate throughout the year. None of the hormones measured, showed a significant fluctuation over the year. No differences were found between the male and female athletes. In females, IGF-BP3 increased significantly $(P<0.01)$ during test 3 . The small non-significant changes in maximal workload throughout the year did not correlate with the hormone values. The athletes showed considerable inter-individual difference. Taken together, it can be concluded that in elite triathletes, who were well-trained all year around, no fluctuations in plasma hormones from the HPA could be observed throughout the year.

\section{Introduction}

Acute stress results in activation of the hypothalamic-pituitary adrenal (HPA) and somatotropic axis, whereas repeated stress leads to adaptation of the HPA axis $(9,20)$. This adaptation is stressor specific, i.e. the HPA axis becomes less responsive to the same stress (10). Severe stress, however, leads to corticoid receptor down regulation in the hippocampus, i.e. diminishes the corticoid negative feed-back (21).

Physical exercise is considered to be a powerful stressor of the HPA and somatotropic axis, as well (6). Exercise training above the anaerobic threshold lowers the plasma adrenocorticotropic hormone (ACTH) and cortisol response to a standard sub-maximal exercise bout $(4,11,12,18)$. In contrast, Wittert and co-workers (1996) showed significantly increased plasma ACTH-, but no different cortisol levels 3-5 days after about 12 hours of strenuous exercise compared with a moderately trained, nonexercised control group (24). In this case, one might hypothesize that a transient over-excitation of the HPA axis have occurred due to the enormous amount of exercise stress. But these conflicting results may also be explained by the selection of subjects (age, sex and the level of 
physiological fitness), the type of sport, and the large inter-individual variability $(8,17,18,23)$.

Notwithstanding the complexity of the endocrine system and our poor understanding of its role during exercise, in sport practice hormones are often used as markers to evaluate the training status of an athlete.

Typically, single measurements of anabolic \{testosterone, $\mathrm{GH}$, insulin-like growth factor 1 (IGF-1), IGF-binding protein 3 (IGF-BP3)\} and catabolic (ACTH, cortisol) hormones are carried out. Since plasma hormone concentrations are prone to rhythmic variations (circadian, ultracadian, etc) and often are secreted in a pulsatile manner, interpretation of nonstandarized single measurements may lead to misinterpretation of the data. Therefore, it is mandatory to obtain endocrine values throughout the year under strictly standardized conditions. If these data are of any significance for evaluation of (too much) training stress is still under debate. The information about seasonal (year round) and eventually training-induced changes in plasma hormone values is limited.

There are some studies in which hormonal changes throughout a period of training and/or tapering were investigated $(3,13,19)$. These studies were all done in competitive swimmers, and only one study in endurance athletes investigated hormonal behavior all year round (7). However, no long term hormonal data are available for multi discipline sports like triathlon. Therefore, the first aim of this study was to investigate the behavior of the hormones \{rest, and (sub)-maximal exercise \} of the hypothalamic-pituitary axis (HPA) under strictly controlled conditions during the different phases of a competitive season in elite triathletes, at rest and during physical exercise. The second aim was to investigate whether changes in training load parallel changes in plasma hormone values.

\section{Material and methods}

\section{Subjects}

Four female and four male elite Olympic distance (OD) triathletes (table 1) who were in full preparation for the oncoming Sydney Olympic 2000 games participated in this longitudinal study, which was approved by the Medical Ethical Committee of the Maastricht University. All subjects were within the top 100 of the ITU ranking and some of them were former European- World champions in one of the triathlon disciplines (e.a Olympic or middle distance). 


\section{TABLE 1}

Physiological characteristics of the male and female athletes at the start of the study.

\begin{tabular}{|lccccc|}
\hline subject & $\begin{array}{c}\text { Age } \\
\text { (years) }\end{array}$ & $\begin{array}{c}\text { Height } \\
(\mathrm{cm})\end{array}$ & $\begin{array}{c}\text { Body mass } \\
(\mathbf{k g})\end{array}$ & $\begin{array}{c}\text { Body fat } \\
(\%)\end{array}$ & $\begin{array}{c}\mathrm{VO}_{2} \text { max } \\
\left(\mathrm{ml}^{\mathrm{m}} \mathrm{kg}^{-1} \cdot \mathrm{min}^{-1}\right)\end{array}$ \\
\hline males & $28.5 \pm 7.9$ & $184 \pm 2.47$ & $2.8 \pm 3.3$ & $11 \pm 2.5$ & $70.2 \pm 6.3$ \\
females & $26.0 \pm 2.2$ & $166 \pm 5.7$ & $56.9 \pm 3.6$ & $18 \pm 3.1$ & $62.1 \pm 2.7$ \\
total & $27.3 \pm 5.5$ & $175 \pm 10.1$ & $64.9 \pm 9.1$ & $15 \pm 4.7$ & $66.1 \pm 6.3$ \\
\hline
\end{tabular}

Values are presented as mean $\pm S D$

\section{General procedure}

As part of a total scientific support program, eight members of the Dutch national Olympic Distance (OD) triathlon team were studied during the year preceding the Sydney Olympic Games. Throughout the year the subjects underwent four times a cycle ergometer test in the laboratory: one during the off season (test 1 ), two during the training season (test 2 during the general preparation phase and test 3 during the specific preparation phase including two altitude stages) and one during the race season (test 4). All subjects were asked to refrain from intense exercise activities at least 24 hours preceding the tests. The subjects were asked to consume the same breakfast for each test session. All testing were done in the morning and for each subject all measurements took place at the same time in similar environmental conditions $\left(22 \pm 1.2^{\circ} \mathrm{C}, 50 \pm 12 \%\right.$ humidity). More than $90 \%$ of the measurements were also undertaken on the same day of the week.

\section{Maximal exercise test}

The subjects had to complete an incremental exercise test on an electro magnetically braked cycle ergometer (Lode Excalibur Sport, Lode, Groningen, The Netherlands) that was calibrated at rpm values between 70 and $100 \mathrm{rpm}$. The test consisted of three stages; an incremental stage towards a steady state, a subsequent steady state level followed by a second incremental stage until exhaustion. The initial workload of the maximal workload test for the male subjects was set at $1.5 \mathrm{~W} / \mathrm{kg}$ body weight (BW) ( $1 \mathrm{~W} / \mathrm{kg}$ BW for females), were after increased by $1 \mathrm{~W} / \mathrm{kg}$ BW every three minutes until a workload of $4.5 \mathrm{~W} / \mathrm{kg}$ BW $(3.5 \mathrm{~W} / \mathrm{kg}$ BW for females) was reached. At this level, the workload was maintained for twelve minutes to ensure a steady state. After the twelve minutes lasting step, the workload was increased again every three minutes by $0.5 \mathrm{~W} / \mathrm{kg}$ 
BW (for both the females and males) until volitional exhaustion occurred. The maximal workload ( $\mathrm{Wmax}$ ) was defined as the last completed stage plus the fraction of time spent in the final load step multiplied by the increase in workload of the last step (16).

Before the start of each test a Teflon catheter was inserted in an antecubital forearm vein followed by a rest for $20 \mathrm{~min}$ in supine position. Blood samples for hormones (ACTH, cortisol, testosterone, growth hormone, IGF-1 and IGF BP3) were drawn at rest, at the end of a $12 \mathrm{~min}$ steady state plateau and directly after completion of the test blood samples for ACTH, cortisol and growth hormone were taken as well.

\section{Blood sample processing and analysis}

Samples $(4 \mathrm{ml})$ for red and white blood cell determination were collected in EDTA tubes, and analysed immediately after (Coulter MD18, Maimi, USA). Four ml of blood was collected in serum tubes for Cortisol, Growth Hormone, IGF-1 and IGF-BP3 analysis, The sampled blood were allowed to clot at room temperature, where after centrifuged at $5,000 \mathrm{rpm}$ at $4^{\circ} \mathrm{C}$. The resulting serum was collected and stored at $-20^{\circ} \mathrm{C}$. Samples for ACTH were collected in pre-cooled EDTA tubes and immediately centrifuged at $5000 \mathrm{rpm}$ at $4^{\circ} \mathrm{C}$. Plasma for $\mathrm{ACTH}$ was stored at $-20^{\circ} \mathrm{C}$. All samples from one subject were analysed in the same run to minimize interassay variability. Sequential Immunometric Assays were used to determine blood concentrations of cortisol (Chiron Diagnostics Corporation, East Walpole, MA, USA), GH (Nichos Institute Diagnostics San Juan Capistrano, CA, USA) and ACTH (Euro/DPC, United Kingdom). Variances for the chemiluminescence assays were: cortisol; intra-assay $4.5 \%$, inter-assay $6.4 \%, \mathrm{GH}$; intra-assay $4.0 \%$, inter-assay $9.2 \%$, and ACTH; intra-assay $5.9 \%$, inter-assay $3.9 \%$. Quantification of IGF-1 was performed using an Immunoradiometric Assay (IRMA) (DSL-5600 ACTIVE"', DSL Deutschland $\mathrm{GmbH}$, Germany) with an intra-assay variance of $3.0 \%$ and an inter-assay of $1.5 \%$. IGFBP-3 was measured by immunoradiometric Assay (IRMA) (DSL-6600 ACTIVE'", DSL Deutschland GmbH, Germany) with an intraassay variance of $3.2 \%$ and an inter-assay of $0.5 \%$.

\section{Statistical analyzes}

All data are presented as mean \pm standard deviation. Statistical analyzes for changes throughout the season were accomplished using a one-way analysis of variance. Where appropriate, a Tukey post hoc test was performed to determine the location of significant difference between the means. Male and female data were separately analyzed. Linear regression was used to determine the Pearson product moment correlation between hormone values and maximal workload or maximal lactate. $\mathrm{P}<0.05$ was considered as statistically significant. 


\section{Results}

The mean $( \pm S D)$ maximal workload and maximal lactate values of both the male and female athletes obtained during the four test sessions are presented in table 2 . A small non significant increase in mean maximal workload (Wmax) $(P=0.06)$ was observed throughout the training season. Mean maximal lactate values did not change over the year.

\section{TABLE 2}

Mean maximal values of the lactate and workload.

\begin{tabular}{|ccccc|}
\hline & TEST 1 & TEST 2 & TEST 3 & TEST 4 \\
\hline Max Lactate (mmol/I) & & & & \\
male & $12.1 \pm 5.7$ & $12.4 \pm 1.9$ & $12.7 \pm 2.3$ & $11.9 \pm 2.8$ \\
female & $9.6 \pm 0.9$ & $8.9 \pm 1.7$ & $10.6 \pm 0.5$ & $10.2 \pm 2.5$ \\
total & $11.0 \pm 4.3$ & $10.7 \pm 2.5$ & $11.5 \pm 1.8$ & $11.1 \pm 2.6$ \\
Max workload (W) & & & & \\
male & & & $392 \pm 23$ & $392 \pm 29$ \\
female & $359 \pm 21$ & $364 \pm 34$ & $278 \pm 21$ & $262 \pm 19$ \\
total & $259 \pm 29$ & $262 \pm 15$ & $327 \pm 64$ & $327 \pm 73$ \\
\hline
\end{tabular}

Values are presented as mean \pm SD

In the female athletes, from all blood variables measured during rest, sub maximal and maximal exercise, only the resting values of the binding protein IGF-BP3 were different (increased) between test period two and four $(P<0.01$ ) (fig 1). No differences were found in plasma ACTH, cortisol, testosterone, TSH, IGF-1 and GH values between the four testing periods. The male athletes showed no significant difference in any of the hormones during rest or (sub) maximal exercise. The delta difference between the resting hormone values and the hormone values obtained during maximal exercise intensity also failed to show any significant difference between the four test sessions in both male and female athletes. Mean maximal workload during the different periods throughout the year did not correlate with hormone values during rest or maximal exercise. Also no correlation was found between resting or maximal hormone values and the maximal lactate values obtained during the incremental exercise test. In addition a considerable intra- and inter-individual variation was observed in resting hormone values and responses to acute exercise. 


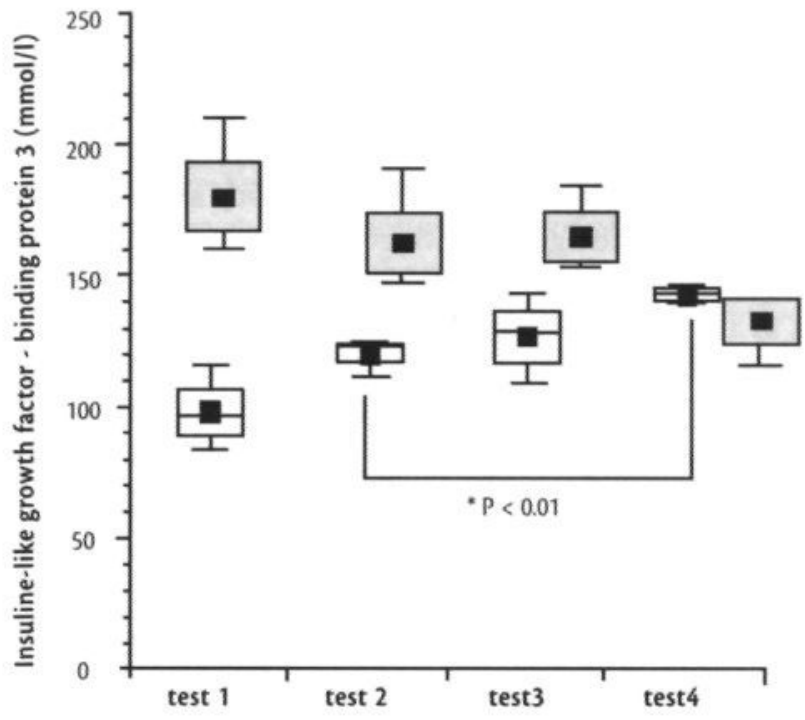

\section{FIGURE 1}

Box-plot of the binding protein IFG-BP3 in the male (gray box) and female (white box) athletes throughout the season.

\section{Discussion}

To evaluate the 'normal' behavior of some hormones, in the present study we examined plasma hormone values at rest and the responses to exercise in male and female elite triathletes during the off-season, training season and the race season and their correlation to performance. The results of present study show that in elite endurance athletes throughout a year mean ACTH, cortisol, testosterone, growth hormone and IGF-1 do not change significantly during at rest or during (sub-) maximal exercise. Unlike in the male athletes, in the female athletes a significant fluctuation over the year of IGF-BP3 was observed, which indicates a change in free IGF-1.

Hormones are known to show a circa-annual cycle $(5,15)$. For instance cortisol values are known to be highest during fall and lowest during spring. This fluctuation is attributed to changes in the light-dark cycles during a year (22). The absence of a circa-annual rhythm in the present study can be the result of adaptation to exercise. In animal studies (9) it has been shown that repeated acute and chronic exposure to a stressor (exercise) results in a less responsive HPA axis and it was found that this adaptation is stressor-specific (10). In humans, chronic intense exercise 
training also results in an adaptation of the hypothalamic-pituitary axis (HPA) $(11,12,18)$. Wittert et al showed that intense physical training led to adaptive changes in basal HPA function, including a phase shift and increased ACTH secretion and a blunted adrenal cortisol response (24). This finding could not be confirmed in the present study and our findings are more in line with the findings of Koziris et al who also found stable cortisol levels during 4 months of training. Increase, in IGF-1 and IGF-BP3 concentrations as a result of endurance training are described as well $(1,13)$. In the study of Koziris and co workers IGF-1 and IGF-BP3 increased significantly during 4 months of training in male and female swimmers, whereas the female swimmers had significantly higher IGF-1 levels compared to the male swimmers (13). IGF-BP3 however, was higher in men after the four months of training. These results are in contrast to the present study were the female athletes showed a significant increase in IGF-BP3 towards the race season, which may have decreased the free IGF-1 levels.

The absence of a significant reduction in hormone response (delta difference rest-max) in reaction to an acute bout of exercise after a period of exercise training as found in some other studies $(3,14)$, is probably also a result of adaptation to long periods of endurance training in these elite athletes. Evidence for this notion can be found in the unchanged maximal performance over the year of the subjects in this study. Furthermore, it should be taken into account that all the subjects involved in the present study maintained an active lifestyle even during the off season which may partly explain the absence of fluctuations during the year. On the other hand it can not be excluded that the training load of these athletes could have been more strenuous, leading to a higher increase in performance (and then maybe to significant fluctuation in hormonal values).

The absence of any significant correlation between the hormone response and the absolute maximal workload or max lactate obtained during the maximal exercise test however, supports the findings of other studies in this area $(3,4)$.

This finding and the large inter-individual variation in the measured hormones during rest and maximal exercise do not support the suggestion that hormones are appropriate markers of training stress and overtraining. From all longitudinal studies (from 12 weeks till one year) done in this field only one study showed a significant alteration of ACTH (decrease) and growth hormone (increase) response to exercise (3). Other failed to find any change in $\operatorname{TSH}(19)$, testosterone $(2,7,19)$, cortisol $(7,19)$, growth hormone (2) or IGF-1 (13). The absence of significant and unequivocal changes of plasma hormone values might be explained by the striking intra-individual variation. Unlike in sedentary subjects, the athletes of the present study did not show a robust and repeatable pattern over time, 
most probably due to the fact that they are very well trained all year around. Such an extremely variable response to exercise has been described earlier by Viru et al. (23). The finding that plasma hormone responses to standardized exercise are hardly repeatable is, to the best of our knowledge, new. Whether this is a normal adaptation to training of a mal-adaptation due to enormous amount of training remains to be established. The results of the present study do support the findings of the latter group of studies and from this it can be concluded that, despite large fluctuations in training load and seasonal influences, in these welltrained athletes no changes in resting and acute exercise induced hormone values were observed.

A novel, albeit isolated, finding in this study was that the reaction of IGFBP3 to endurance exercise is different for men and women.

\section{Acknowledgement}

This study was supported by a grant from the Netherlands Olympic Committee (NOC*NSF). The authors wish to acknowledge the cooperation of the triathletes and the support from Dr. Eric van Breda, Louis Delahaye, Dennis van Hamont, Ingrid van de Port, Dotje Hennekes and Kirsten Frick during all the testing. 


\section{References}

1. Bang P., J. Brandt, M. Degerblad, G. Enberg, L. Kaijser, M. Thoren, K. Hall. Exerciseinduced changes in insulin-like growth factors and their low molecular weight binding protein in healthy subjects and patients with growth hormone deficiency. Eur J Clin Invest 20: 285-292. 1990.

2. Bell G.J., D. Syrotuik, T.P. Martin, R. Burnham, H.A. Quinney. Effect of concurrent strength and endurance training on skeletal muscle properties and hormone concentrations in humans. Eur J Appl Physiol 81: 418-427. 2000.

3. Bonifazi M., E. Bela, C. Lupo, G. Martelli, B. Zhu, G. Carli. Influence of training on the response to exercise of adrenocorticotropin and growth hormone plasma concentrations in human swimmers. Eur J Appl Physiol Occup Physiol 78: 394-397. 1998.

4. Buono M.J., J.E. Yeager, A.A. Sucec. Effect of aerobic training on the plasma ACTH response to exercise. J Appl Physiol 63: 2499-2501. 1987.

5. Cooke R.R., J.E. McIntosh, R.P. McIntosh. Circadian variation in serum free and non-SHBG-bound testosterone in normal men: measurements, and simulation using a mass action model. Clin Endocrinol (Oxf) 39: 163-171. 1993.

6. Galbo $\mathrm{H}$. The hormonal response to exercise. Diabetes Metab Rev 1: 385-408 1986.

7. Hakkinen K., K.L. Keskinen, M. Alen, P.V. Komi, H. Kauhanen. Serum hormone concentrations during prolonged training in elite endurance-trained and strength-trained athletes. Eur J Appl Physiol Occup Physiol 59: 233-238 1989.

8. Hakkinen K., A. Pakarinen, M. Alen, H. Kauhanen, P.V. Komi. Neuromuscular and hormonal adaptations in athletes to strength training in two years. J Appl Physiol 65: 2406-2412. 1988.

9. Hashimoto K., S. Suemaru, T. Takao, M. Sugawara, S. Makino, Z. Ota. Corticotropin-releasing hormone and pituitary-adrenocortical responses in chronically stressed rats. Regul Pept 23: 117-126. 1988.

10. Kant G.J., T. Eggleston, L. Landman-Roberts, C.C. Kenion, G.C. Driver, J.L. Meyerhoff. Habituation to repeated stress is stressor specific. Pharmacol Biochem Behav 22: 631-634. 1985.

11. Keizer H.A., H. Kuipers, J. de Haan, E. Beckers, L. Habets. Multiple hormonal responses to physical exercise in eumenorrheic trained and untrained women. Int J Sports Med 8 Supp/ 3: 139-150. 1987.

12. Keizer H.A., H. Kuipers, J. de Haan, G.M. Janssen, E. Beckers, L. Habets, G. van Kranenburg, P. Geurten. Effect of a 3-month endurance training program on metabolic and multiple hormonal responses to exercise. Int / Sports Med 8 Suppl 3: 154-160. 1987.

13. Koziris L.P., R.C. Hickson, R.T. Chatterton, Jr., R.T. Groseth, J.M. Christie, D.G. Goldflies, T.G. Unterman. Serum levels of total and free IGF-I and IGFBP-3 are increased and maintained in long-term training. J Appl Physiol 86: 1436-1442 1999.

14. Kraemer W.J., S.J. Fleck, R. Callister, M. Shealy, G.A. Dudley, C.M. Maresh, L. Marchitelli, C. Cruthirds, T. Murray, J.E. Falkel. Training responses of plasma beta-endorphin, adrenocorticotropin, and cortisol. Med Sci Sports Exerc 21: 146153. 1989. 
15. Krieger D.T., W. Allen, F. Rizzo, H.P. Krieger. Characterization of the normal temporal pattern of plasma corticosteroid levels. J Clin Endocrinol Metab 32: 266-284. 1971.

16. Kuipers H., F.T.J. Verstappen, H.A. Keizer, P. Geurten, G. Kranenburg van. Variability of Aerobic Performance in the Laboratory and Its Physiologic Correlates. Sport Med 6: 197-201 1985.

17. Lehmann M., U. Gastmann, K.G. Petersen, N. Bachl, A. Seidel, A.N. Khalaf, S. Fischer, J. Keul. Training-overtraining: performance, and hormone levels, after a defined increase in training volume versus intensity in experienced middle- and long-distance runners. Br J Sports Med 26: 233-242. 1992.

18. Luger A., P.A. Deuster, S.B. Kyle, W.T. Gallucci, L.C. Montgomery, P.W. Gold, D.L. Loriaux, G.P. Chrousos. Acute hypothalamic-pituitary-adrenal responses to the stress of treadmill exercise. Physiologic adaptations to physical training. N Engl J Med 316: 1309-1315. 1987.

19. Mujika I., J.C. Chatard, S. Padilla, C.Y. Guezennec, A. Geyssant. Hormonal responses to training and its tapering off in competitive swimmers: relationships with performance. Eur J Appl Physiol Occup Physiol 74: 361-366 1996.

20. Sapolsky R.M., L.C. Krey, B.S. McEwen. The neuroendocrinology of stress and aging: the glucocorticoid cascade hypothesis. Endocrinology Reviews 7: 284-301 1986.

21. Sapolsky R.M., B.S. McEwen. Down-regulation of neural corticosterone receptors by corticosterone and dexamethasone. Brain Res 339: 161-165. 1985.

22. Touitou Y., O. Benoit, J. Foret, A. Aguirre, A. Bogdan, M. Clodore, C. Touitou. Effects of a two-hour early awakening and of bright light exposure on plasma patterns of cortisol, melatonin, prolactin and testosterone in man. Acta Endocrinol (Copenh) 126: 201-205. 1992.

23. Viru A., K. Karelson, T. Smirnova. Stability and variability in hormonal responses to prolonged exercise. Int J Sports Med 13: 230-235 1992.

24. Wittert G.A., J.H. Livesey, E.A. Espiner, R.A. Donald. Adaptation of the hypothalamopituitary adrenal axis to chronic exercise stress in humans. Med Sci Sports Exerc 28: 1015-1019 1996. 



\section{CHAPTER 5}

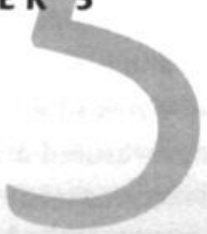

\section{Physiological variables and lactate indices}

in incremental exercise tests with different stage duration

This Chapter has been submitted as:

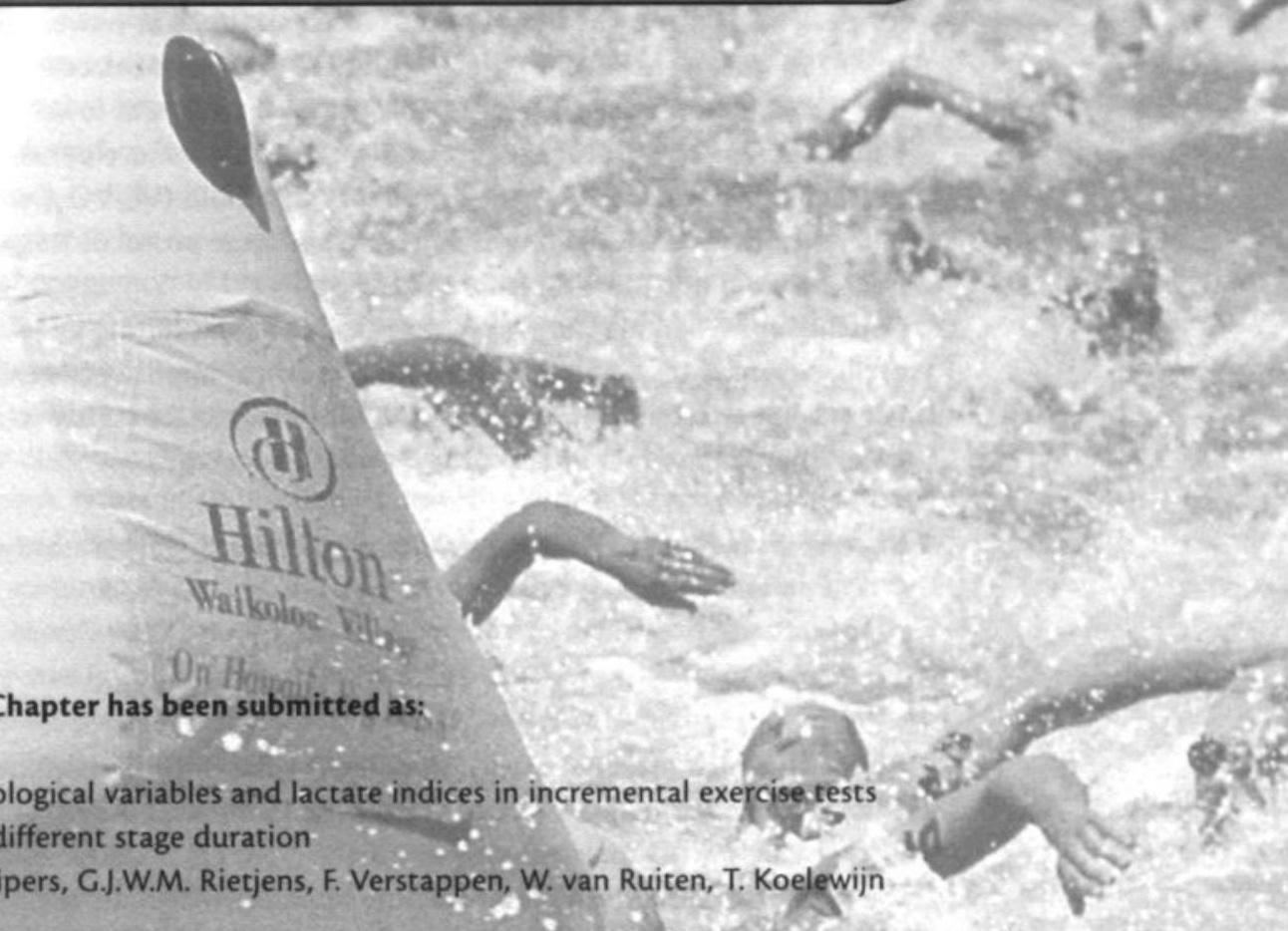

Physiological variables and lactate indices in incremental exercise.tests with different stage duration

H. Kuipers, G.J.W.M. Rietjens, F. Verstappen, W. van Ruiten, T. Koelewijn

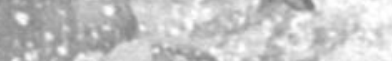

Nutrition añ Toxicology Research Institute Maastricht (NUTRIM)

$\therefore$ Deparcinent of Movements Sciences, Maastricht University, Maascrich 5 The Netherlands

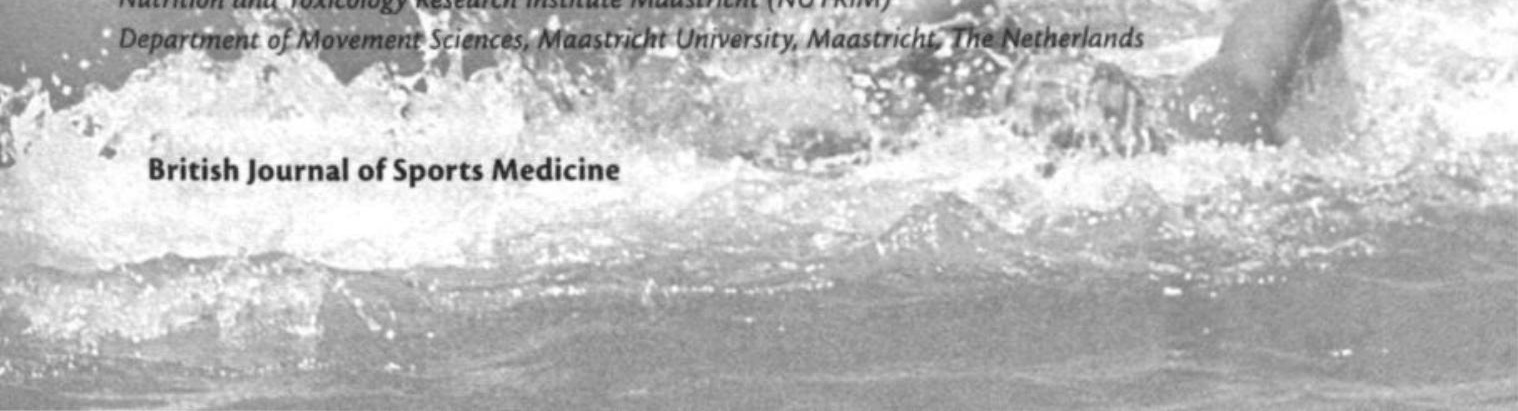




\section{Abstract}

Objectives: To study the effect of stage duration on physiological variables in an incremental exercise test, ten well-trained cyclists underwent 3 graded exercise tests with stage duration of $1,2.5$ and 5 minutes.

Methods: Respiratory variables and heart rate were measured in the 1, 2.5 and 5 minutes protocols, while blood lactate was measured only in the protocol with 2.5 and 5-minute stages. Maximal lactate steady state was assessed in a different 4 th test, consisting of three 15 minutes workout based on the power output at which blood lactate was $4 \mathrm{mmol} / \mathrm{l}(\mathrm{P} 4)$ in the 5 minute per stage incremental protocol (25 watt below P4, at P4 and 25 watt above P4). Results: Maximal power output (Pmax) was lower in the 5 minutes stage protocol, compared with the 1-minute stage protocol ( 312 versus 388 watt). Peak $\mathrm{VO}_{2}$ and peak heart rate were lower in the 5-minute stage protocol compared with the 1-minute stage protocol $\left(4.73 \mathrm{I} / \mathrm{min}\right.$ versus $4.91 \mathrm{I} / \mathrm{min}$ for $\mathrm{VO}_{2}$ peak and 188 versus 194 beats $/ \mathrm{min}$ for peak heart rate). The blood lactate concentrations at power outputs from 250 watt were lower in the 2.5 -minute stage protocol, compared with the 5 minutes per stage protocol. The $\mathrm{P} 4 \mathrm{mmol} / \mathrm{I}$ was lower in the 5 minute per stage protocol compared with the 2.5 minute per state protocol (253 versus 270 watt). Ventilatory threshold $\left(\mathrm{VE} / \mathrm{VO}_{2}\right)$ was different between the 1, 2.5 and 5 minute per stage protocol. Respiratory frequency did not show threshold behavior.

Conclusion: When measuring physiological variables during incremental cycling exercise tests at maximal and sub maximal intensity the duration per stage has to be taken into account. Maximal lactate steady state can be estimated based on $\mathrm{P} 4$ from the 5 minute per stage protocol.

Key words: cycling, peak $\mathrm{VO}_{2}$, ventilatory threshold, lactate steady state

\section{Introduction}

Although a high maximal oxygen uptake $\left(\mathrm{VO}_{2} \mathrm{max}\right)$ is a prerequisite for endurance performance, exercise beyond 10-15 minutes is performed at an intensity below the maximal oxygen uptake $(7,8,17)$. Studies, investigated the relationship between plasma lactate response, $\mathrm{VO}_{2}$ max, and endurance performance, have shown that endurance performance is correlated higher with lactate variables such as anaerobic threshold rather than with $\mathrm{VO}_{2} \max (5,14,19,21)$.

A point that has received little attention with respect to plasma lactate measurements in literature is that, in contrast to ventilatory variables, it may take at least 3 minutes in an incremental exercise test $(15,16)$ and 
10-15 minutes in a single load test, to establish steady plasma lactate concentrations at a given power output $(3,9)$. This would imply that studies on lactate measurements in which stages of less than 3 minutes were used, may have failed to yield steady plasma lactate concentration, at each exercise intensity.

A more recently proposed variable with respect to lactate response is the maximal lactate steady state (maxLASS), defined as the highest constant exercise intensity at which the plasma lactate does not change more than $1 \mathrm{mmol} / \mathrm{l}$ during minute 10 en 30 (12). A major drawback of the assessment of maxLASS is that the procedure is time consuming because it takes several exercise bouts of at least $\mathbf{3 0}$ minutes duration each. Therefore, there is a need for less time consuming procedures to assess maxLASS. As has been shown by Foxdal et al. (9) the longer each exercise intensity lasts in incremental exercise tests, the more reliable will be the determination of the lactate response. However, with prolonging the duration of the stages maximal values of oxygen uptake, heart rate, and some other variables may not be reached.

In addition to measurements of the lactate response, Cheng et al. (6) reported that breathing frequency also shows threshold characteristics when plotted as function of exercise intensity. However, breathing frequency is difficult to measure outside the laboratory, and therefore little research has been conducted to assess the usefulness of the threshold phenomenon of breathing frequency as indicator for endurance capacity. In addition its relationship with lactate variables has to be established yet.

These various questions led to the present study in which our first aim was to compare the response of some key physiological variables as well as their peak values in an incremental exercise protocol that was done three times, and in which the duration per stage was different ( $1 \mathrm{~min}, 2.5 \mathrm{~min}$, and 5 minutes). A second aim was to relate the various lactate and ventilatory thresholds obtained in the different protocols with the maximal lactate steady state. A third aim was to investigate whether respiratory frequency does show threshold behavior and if so, whether it is related to other threshold variables or maxLASS.

\section{Materials and methods}

\section{Subjects}

After approval from the local ethical committee 6 male and 4 female welltrained cyclists participated after written informed consent. All subjects trained at least 4 times per week for at least one hour per training session. The mean age was $26 \pm 11$ years (range 18-54), mean weight $69 \pm 6 \mathrm{~kg}$, mean length $180 \pm 8 \mathrm{~cm}$. 


\section{Exercise protocols}

The study took place in springtime, and all subjects had been in training for the oncoming season for at least two months. For studying the physiological response in an incremental exercise test with different duration per stage 3 incremental exercise protocols were performed. The order of the first three protocols was random, and at least one week separated two consecutive tests.

Protocol A started at 100 watt (W) for one minute, followed by a stepwise increase of $20 \mathrm{~W}$ every single minute until the subject was unable to continue.

Protocol B started at $100 \mathrm{~W}$, after which every 2.5 minute the power output was increased by $50 \mathrm{~W}$, and from a heart rate of approximately 155 with 25 watt every 2.5 minute.

Protocol $\mathrm{C}$ was similar to protocol $\mathrm{B}$, with the only difference that every stage lasted 5 minutes.

If in protocols $B$ and $C$ a stage could not be completed to the full length the maximal power output (Pmax) was calculated according to the equation:

\section{Pmax $=$ Pcompleted + Tcompleted $/$ Tstage $*$ load increment}

in which Pcompleted is the power output of the last stage that was completed, Tcompleted the number of seconds that the uncompleted power output could be sustained, Tstage the number of seconds required to complete the stage, and load increment is the last load increment. To assess maximal lactate steady state, a fourth protocol (D) was conducted, and consisted of 3 incremental workloads each lasting 15 minutes, preceded by a 5 minute warm up at $50 \%$ of the previously assessed maximal power output attained in the 5 minute per stage protocol. The three intensities of protocol $D$ were based on the power output from protocol $C$ at which the blood lactate concentration was $4 \mathrm{mmol} / \mathrm{l}(\mathrm{P} 4)$. The first power output was 25 watt below P4, the second at P4, and the third 25 watt above P4.

\section{Procedures}

After the subjects reported to the lab, a venous catheter was inserted into a forearm vein. A stopcock was connected to the catheter, to enable collection of blood. The subject was connected to a heart rate monitor (Polar), and wore a facemask, connected to a portable metabolic system (Cosmed K4), and subsequently mounted the cycle ergometer (Lode Excalibur, The Netherlands). 


\section{Variables}

Respiratory variables and heart rate were measured continuously, and displayed every 30 seconds on a portable computer, that was connected to the receiving unit of the $\mathrm{K} 4$ metabolic system.

From the respiratory variables the ventilatory threshold, based on $\mathrm{VE} / \mathrm{VO}_{2}$ was determined by the software provided with the K4 measuring equipment. The ventilatory threshold was defined as the power output at which the $\mathrm{VE} / \mathrm{VO}_{2}$ started to depart from linear increase. In addition a break point in the linear increase of the ventilation rate was searched for by regression analyses.

From the venous catheter a $2 \mathrm{ml}$ blood sample was drawn during the final 30 seconds of every workload, except for protocol A with increments every minute in which no blood samples were taken. In protocol D, with 15-minute stages for assessing maxLASS blood was sampled every 5 minutes. The first $0.5 \mathrm{ml}$ of the sample was discarded because of dilution with saline. After sampling the catheter was flushed with saline. The blood lactate concentration was measured immediately after sampling (EML-105, Radiometer, Copenhagen, Denmark).

\section{Data handling and statistical analysis}

The data obtained during the last 30 seconds of the various protocols were used for analysis. The data were analyzed with a SPSS software packet analyzed for correlations using a repeated measures ANOVA. Depending on the 'Mauchly's test of sphericity a uni- or multi-variate test was employed. Differences between the protocols were analyzed with a paired t-test. In case of univariate testing a Bonferroni correction was applied. Differences were considered statistically significant at $\mathrm{P}<0,05$.

\section{Results}

The mean peak power output during the incremental protocols was inversely related to the duration of the stages (Table 1). The highest mean peak power output was attained in protocol $A$ in which the load was increased by 20 watt every minute (mean $388 \pm 68$ watt), while the lowest peak power output was attained in the protocol with 5 minute stage ( $312 \pm 48$ watt; $\mathrm{P}<0.05$ compared with the 1 -minute protocol).

Mean peak $\mathrm{VO}_{2}$ (Table 1) in the incremental protocols was highest in the protocol with 1-minute stages, and lowest in the protocol with 5 minutes states $(P<0.05$ between the 1 -minute and 5 -minute protocols).

From table 1 it can be seen that the mean peak heart rate in the protocol with a load increase every minute was $194 \pm 8$ beats $/ \mathrm{min}$, which was significantly higher compared with the 5 minute per stage protocol (188 \pm 68 watt; $\mathrm{P}<0.05$ ). 
The plasma lactate response during the 2,5 and 5 minutes per stage protocols were different, i.e. in all subjects the curve obtained from the 2,5 minutes per stage protocol was shifted to the right compared with the 5 minutes per stage protocol (Figure 1).

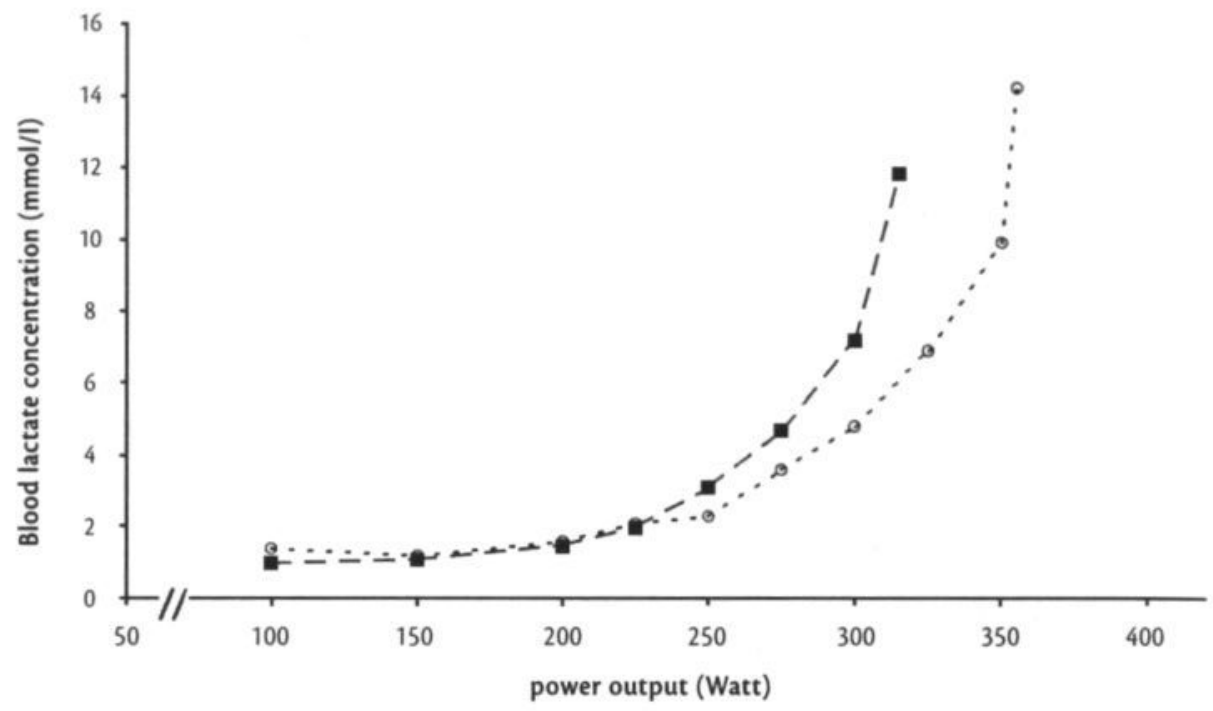

Figure 1

Example of blood lactate concentration of a male subject as function of power output in two different incremental exercise protocols with stages lasting 2.5 minutes (circles), and 5 minutes (squares).

The mean peak blood lactate concentration in the protocol with 2.5 minute stages was higher than in the protocol with 5 -minute stages ( 12.5 versus $11.6 \mathrm{mmol} / \mathrm{l} ; \mathrm{P}<0.05$ ). Since the $4 \mathrm{mmol} / \mathrm{l}$ intensity is often used as parameter for endurance capacity, the power output at which the blood lactate concentration was $4 \mathrm{mmol} / \mathrm{l}$ was assessed in the protocols with 2.5 and 5-minute stages. The power output at which a plasma lactate concentration of $4 \mathrm{mmol} / \mathrm{l}$ was reached was significantly higher in the 2.5 minute per state protocol ( $270 \pm 46$ watt) compared with the 5 minutes per stage protocol ( $253 \pm 41$ watt; $P<0.05)$.

As can be seen from Table 1 the power output at which $\mathrm{VE} / \mathrm{VO}_{2}$ departed from a linear increase was $306 \pm 60$ watt in the 1-minute per stage protocol, $282 \pm 50$ watt in the 2.5 min per stage protocol $(P<0.001$ from 1 -minute protocol), and $265 \pm 49$ watt in the 5 minute stage protocol $(\mathrm{P}<0.001$ compared with the 1 -minute stage protocol, and $\mathrm{P}<0.01$ compared with 2.5 minute stage protocol). 


\section{TABLE 1}

Values (mean \pm sd) of maximal power output ( $P$ max), peak $\mathrm{VO}_{2}$, peak heart rate (PeakHR), peak blood lactate concentration (Peak lactate), the power output at 4 $\mathrm{mmol} / \mathrm{l}$ blood lactate (PO4mmol/I), and the power output at breakpoint $\mathrm{VE} / \mathrm{VO}_{2}$ (Break VE/ $/ \mathrm{O}_{2}$ ) in incremental protocols with stage length of 1 minute (protocol $\mathrm{A}$ ), 2.5 minutes (protocol B), and 5 minutes (protocol C).

\begin{tabular}{|ccccccc|}
\hline $\begin{array}{c}\text { Protocol } \\
\text { (Watt) }\end{array}$ & $\begin{array}{c}\text { Pmax } \\
(1 / \mathrm{min})\end{array}$ & $\begin{array}{c}\text { PeakVO } \\
(\text { beats } / \mathrm{min})\end{array}$ & $\begin{array}{c}\text { Peak HR } \\
(\mathrm{mmol} / \mathrm{l})\end{array}$ & $\begin{array}{c}\text { Peak Lactate } \\
\text { (Watt) }\end{array}$ & $\begin{array}{c}\text { PO4mmol/I Break VE/VO } \\
\text { (Watt) }\end{array}$ & \\
\hline A & $372 \pm 68$ & $4.91 \pm 0.88$ & $194 \pm 8$ & & & $306 \pm 60$ \\
B & $330 \pm 56$ & $4.84 \pm 0.95$ & $190 \pm 7$ & $12.5 \pm 1.7$ & $288 \pm 46$ & $282 \pm 50^{* *}$ \\
C & $298 \pm 48^{*}$ & $4.73 \pm 0.92^{*}$ & $186 \pm 9^{*}$ & $11.6 \pm 2.0$ & $268 \pm 41^{\#}$ & $265 \pm 49 \#$ \\
\hline
\end{tabular}

- $P<0.05$ between $C$ and $A$.

* $P<0.05 B$ compared with $A$

\# $P<0.05$ between $C$ and $B$

\#\# $\mathrm{P}<0.01 \mathrm{C}$ compared with A

Breathing frequency as function of power output failed to show a consistent pattern in the subjects during the different protocols, and no clear threshold behavior could be observed (data not shown).

The power output at $4 \mathrm{mmol} / \mathrm{l}$ blood lactate concentration (P4) in the 5 -minute per stage protocol was $253 \pm 49$ watt, whereas the power output at which the maxLASS was reached in the protocol with 15 minute stages was $254 \pm 48$ watt. The mean blood lactate concentration at maxLASS was $4.89 \pm 0.73 \mathrm{mmol} / \mathrm{l}$ (range between individuals $3.5-5.6 \mathrm{mmol} / \mathrm{l}$ ). All subjects were able to start with the load being 25 watt above P4, however 8 stopped prematurely because of fatigue, while blood lactate concentrations increased progressively. Only 2 subjects were able to finish the 15 minutes with difficulty, and blood lactate levels raised to 8.5 and $10.2 \mathrm{mmol} / \mathrm{l}$.

\section{Discussion}

One of the aims of the present study was to investigate peak values of some physiological variables in an incremental cycle ergometer protocol in dependence of the duration of every stage. The present study demonstrates that in a protocol in which the power output was increased by 20 watt every minute, the maximal power output is on the average 43 watt higher compared to a protocol in which the power output was 
increased every 2,5 minute, and on the average 76 watt higher compared with a protocol in which the power output is increased every 5 minutes. The higher maximal power output in protocols with a short time per stage can be explained by a greater contribution of anaerobic power with shorter load steps, and delayed lactate accumulation associated with local muscle fatigue. Since maximal power output can be used as a parameter for endurance capacity $(13,18)$, the different maximal power outputs in the protocols with different duration per stage have to be taken into account when reporting maximal power outputs, and when comparing power outputs attained in incremental protocols with different duration per stage.

The peak $\mathrm{VO}_{2}$ was also significantly lower in the 5 minute per stage protocol compared with the 1-minute per stage protocol. This can be explained because the adjustment of circulation and oxygen uptake requires approximately one minute, enabling the cardio-respiratory systems to adjust to the corresponding power output in about one minute (2). It is likely that with longer duration local fatigue accumulates, preventing to exploit the full capacity of the cardio-respiratory system. This implies that for measuring $\mathrm{VO}_{2}$ max in an incremental protocol load increases every minute appear to be preferable. Also for measuring peak heart rate a one minute load increment appears to be adequate. The data of the present study demonstrate that for measuring plasma lactate concentrations belonging to a given power output, a longer duration per stage is required. The results of the present study show that when the duration per stage in incremental protocols is shorter than approximately 5 minutes, plasma lactate concentrations lag behind the corresponding power output. This is in line with the findings of Foxdall et al (9) who compared the blood lactate response in incremental treadmill protocols with 4, 6 and 8-minute stages. They concluded that it may take 5-8 minutes before plasma lactate reaches a steady concentration (9). This would also imply that protocols for measuring plasma lactate parameters such as the $4 \mathrm{mmol} / \mathrm{l}$ intensity, load steps less than approximately 5 minutes yield unreliable results. The power output at which blood lactate concentration equals $4 \mathrm{mmol} / \mathrm{l}(\mathrm{P} 4)$ is generally used as a parameter for endurance capacity $(1,5)$. When looking at the power output at $\mathrm{P} 4$ in the incremental protocol with different duration per stage, it was found in the present study that $\mathrm{P} 4$ was on the average 20 watt lower in the protocol with 5 minutes stages, compared with the protocol with 2.5-minute stages. This reflects the insufficient time for lactate equilibration in the incremental protocol with 2.5 minutes stages. One of the important findings of the present study is that maximal lactate steady state can be measured with the combination of an incremental exercise protocol with 5 minute stages and a protocol with three stages 
each lasting 15 minutes. Since maxLASS approximates the P4 in the 5 minute per stage protocol the three power outputs were 25 watt below $\mathrm{P} 4$, at $\mathrm{P} 4$, and 25 watt above $\mathrm{P} 4 \mathrm{mmol} / \mathrm{l}$ measured in the 5 minute per state protocol. The 15 minutes of continuous exercise at a given power output for assessing maximal lactate steady state is shorter than used by other investigators, using 20-30 minute of continuous exercise $(4,12)$. Jones \& Doust proposed to adopt 30 minutes constant exercise for assessing maxLASS in running. However, their results show that when no steady state is reached in approximately 10 minutes the exercise is not at maxLASS. Jenkins and Quigley (11) also observed that blood lactate levels remained stable between 10 and 30 minutes of continuous exercise. In a pilot study before starting the present investigation four subjects were tested with 30-minute stages to assess maxLASS. This confirmed the results of Jenkins and Quigley (11) that between 10 and 30 minutes the blood lactate concentration remains within the $1 \mathrm{mmol}$ variation at maxLASS, in contrast to an intensity of 25 Watt above maxLASS where blood lactate concentration continue to rise throughout the exercise period. Similar results were found in a study during running (20). Therefore, it can be concluded that maxLASS can be approximated by P4 obtained from an incremental protocol with 5-minute stages, and assessed accurately with a protocol consisting of 15 -minute stages and power outputs based on P5 from the 5 minute per stage protocol.

Ventilatory threshold is also used as parameter for endurance capacity. In the present study ventilatory threshold was defined as the power output at which $\mathrm{VE} / \mathrm{VO}_{2}$ started to increase non-linearly. The results of the present study show that the power output at which the non-linear increase in $\mathrm{VE} / \mathrm{VO}_{2}$ started, was lower when the duration per stage was longer (Table 1). With 5 minutes per stage ventilatory threshold is very close to P4. This implies that when ventilatory threshold is used also stages of at least 5 minutes should be used.

Another aim of the present study was to investigate whether breathing frequency showed threshold behavior and could be used as parameter for endurance performance. The results show that breathing frequency failed to show a stable and consistent pattern, and therefore no clear and consistent threshold could be detected in most subjects. This appears to be in contrast to a study by Cheng et al (6), and James et al (10). Cheng et al (1992), however, employed the Dmax method, which always yields some kind of threshold as long as the variable increases non-linearly, irrespective of a real threshold behavior. This is the case with respiratory frequency that increases non-linear, however, fails to show a threshold behavior as blood lactate does. The study of James et al (10) indicates that breathing frequency can be used as an indicator of anaerobic threshold, defined as the disproportionate increase in breathing frequency. However, in the 
present study no consistent disproportionate increase in breathing frequency could be found.

A relevant point for the interpretation of maxLASS power output and other threshold variables is the relationship with endurance performance. A point that has received insufficient attention is the observation that there is no such thing as one critical speed at which endurance performances are done. When the average speeds of world records at different distances in certain disciplines are compared it appears that the average speed declines with increasing distance. This indicates that there is not one single critical speed, but that every distance may have its own critical speed. This is supported by the study from Bishop et al. (5) who studied the relationship between lactate parameters and one hour cycling performance, and with Jones \& Doust (12) who studied the relationship between $8 \mathrm{~km}$ running performance and lactate parameters. In a study by Jenkins \& Quigley (11) cyclists had to exercise for 30 minutes and it was observed that the blood lactate levels remained stable between 10 and 30 minutes and could reach values up to $10 \mathrm{mmol} / \mathrm{l}$. Foster et al (8) studied lactate levels during a simulated time trial over $5 \mathrm{~km}$, and observed that plasma lactate levels increased throughout the trial. Therefore, it can be concluded that lactate threshold variables may be helpful for assessing endurance characteristics in athletes, however have limited value for predicting endurance performance. There appears not to be one critical exercise intensity valid for different distances.

In conclusion, peak $\mathrm{VO}_{2}$ and peak heart rates can be obtained in incremental protocols with increments every minute. When steady blood lactate levels and ventilatory threshold are required the duration per stage should be at least 5 minutes. The maxLASS power output can be estimated from power output at $4 \mathrm{mmol} / \mathrm{l}$ in an incremental protocol with 5 minutes per stage, and can be measured by 3 power outputs around the estimated maxLASS, in which every power output is maintained for 15 minutes.

\section{Acknowledgement}

This work was supported by grants from the Netherlands Olympic Committee and Netherlands Sports Federation (NOC*NSF). 


\section{References}

1. Anderson G.S., E.C. Rhodes. A review of blood lactate and ventilatory methods of detecting transition thresholds. Sports Med 8: 43-55. 1989.

2. Astrand P.O., K. Rohdahl. Textbook of work physiology. New York, London: MacGraw Hill International Editions, 1986.

3. Aunola S., H. Rusko. Does anaerobic threshold correlate with maximal lactate steady-state? J Sports Sci 10: 309-323. 1992.

4. Beneke R., S.P. von Duvillard. Determination of maximal lactate steady state response in selected sports events. Med Sci Sports Exerc 28: 241-246. 1996.

5. Bishop D., D.G. Jenkins, L.T. Mackinnon. The relationship between plasma lactate parameters, Wpeak and 1-h cycling performance in women. Med Sci Sports Exerc 30: $1270-1275.1998$.

6. Cheng B., H. Kuipers, A.C. Snyder, H.A. Keizer, A. Jeukendrup, M. Hesselink. A new approach for the determination of ventilatory and lactate thresholds. Int / Sports Med 13: 518-522. 1992.

7. Costill D.L., H. Thomason, E. Roberts. Fractional utilization of the aerobic capacity during distance running. Med Sci Sports S: 248-252. 1973.

8. Foster C., M.A. Green, A.C. Snyder, N.N. Thompson. Physiological responses during simulated competition. Med Sci Sports Exerc 25: 877-882. 1993.

9. Foxdal P., A. Sjodin, B. Sjodin. Comparison of blood lactate concentrations obtained during incremental and constant intensity exercise. Int J Sports Med 17: 360-365. 1996.

10. James N.W., G.M. Adams, A.F. Wilson. Determination of anaerobic threshold by ventilatory frequency. Int J Sports Med 10: 192-196. 1989.

11. Jenkins D.G., B.M. Quigley. Blood lactate in trained cyclists during cycle ergometry at critical power. Eur J Appl Physiol Occup Physiol 61: 278-283 1990.

12. Jones A.M., J.H. Doust. The validity of the lactate minimum test for determination of the maximal lactate steady state. Med Sci Sports Exerc 30: 1304 . 1313. 1998.

13. Lucia A., J. Pardo, A. Durantez, J. Hoyos, J.L. Chicharro. Physiological differences between professional and elite road cyclists. Int / Sports Med 19: 342-348. 1998.

14. Maffulli N., G. Capasso, A. Lancia. Anaerobic threshold and performance in middle and long distance running. J Sports Med Phys Fitness 31: 332-338. 1991.

15. McLellan T.M. Ventilatory and plasma lactate response with different exercise protocols: a comparison of methods. Int / Sports Med 6: 30-35. 1985.

16. Moreau K.L., M.H. Whaley, J.H. Ross, L.A. Kaminsky. The effects of blood lactate concentration on perception of effort during graded and steady state treadmill exercise. Int J Sports Med 20: 269-274. 1999.

17. Morgan D.W., J.T. Daniels. Relationship between VO2max and the aerobic demand of running in elite distance runners. Int / Sports Med 15: 426-429. 1994.

18. Mujika I., S. Padilla. Physiological and performance characteristics of male professional road cyclists. Sports Med 31: 479-487 2001.

19. Nicholson R.M., G.G. Sleivert. Indices of lactate threshold and their relationship with 10-km running velocity. Med Sci Sports Exerc 33: 339-342. 2001. 
20. Rietjens G.J.W.M., H. Kuipers, F.T. Verstappen, H. Schoenmakers, G. Hofman. Stage duration in incremental running tests, effects on physiological variables and lactate indices. Br J Sport med: submitted.

21. Shephard R.J. Muscular endurance and blood lactate. London: Blackwell, 1992. 
CHAPTER 6

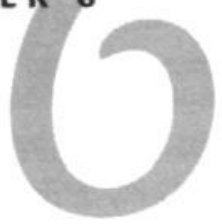

Effects of stage duration in incremental running tests, effects on physiological variables

This chapter has been accepted for publication as:

Effects of stage durantion in incremental running tests, effects on physiological variables

G. Rietjens, H. Kuipers, F. Verstappen, H. Schoenmakers, G. Hofman

Nutrition and Toxicology Research Institute Maastricht (NUTRIM)

Department of movement sciences, University Maastricht, Maastricht, The Netherlands

British Journal of Sports Medicine 


\section{Abstract}

Objective: For studying the effect of stage duration on peak values of physiological variables and threshold phenomena in an incremental running test, 8 well-trained runners underwent 3 identical maximal incremental running tests with the only difference that stage duration was 1,3 and 6 minutes.

Methods: Respiratory variables, and heart rate were measured during the final 30 seconds of every stage, and blood lactate only in the protocol with 3 and 6 minute stages. In order to study maximal lactate steady state (maxLASS) and its corresponding speed, every subject underwent a $4^{\text {th }}$ test in which three 15 minutes stages at three different running speeds, based on the running speed at $4 \mathrm{mmol} / \mathrm{l}$ blood lactate (V4) in the 6 minute per stage protocol. The first load in the 15 minute per stage test was $\mathrm{V} 4$ minus $0.5 \mathrm{~km} / \mathrm{hr}$, at $\mathrm{V} 4$, and the third $\mathrm{V} 4$ plus $0.5 \mathrm{~km} / \mathrm{hr}$. To compare the blood lactate concentration at maxLASS speed on the treadmill and in the field, the subjects also ran $5 \mathrm{~km}$ at this speed on an outdoor tartan track after which blood lactate was measured.

Results: The results show that maximal running speed (Vmax) in the incremental test was significantly lower in the 6 minutes stage protocol, compared with the 1 minute stage protocol ( 15.5 versus $18.3 \mathrm{~km} / \mathrm{hr}$ ). Peak $\mathrm{VO}_{2}$ and peak heart rate were not different between in the 6 minute stage protocol and the 1 minute stage protocol. The $\mathrm{V} 4$ was significantly lower in the 6 minute per stage protocol compared with the 3 minute per stage protocol ( 12.9 versus $14.4 \mathrm{~km} / \mathrm{hr})$. Ventilatory threshold $\left(\mathrm{VE} / \mathrm{VO}_{2}\right)$ was not different between the 1, 3 and 6 minute per stage protocol. Maximal lactate steady state can be estimated from $\mathrm{V} 4$ in the 6 minute per stage protocol, and verified by three 15 minute intensities being $\mathrm{V} 4$ minus 0.5 $\mathrm{km} / \mathrm{hr}$, at $\mathrm{V} 4$, and $\mathrm{V} 4$ plus $0.5 \mathrm{~km} / \mathrm{hr}$. The blood lactate concentration at the maxLASS speed was not different between treadmill running and running on an outdoor track.

Conclusion: When measuring lactate variables during incremental exercise tests at maximal and submaximal intensity the duration per stage has to be taken into account.

Key words: anaerobic threshold, ventilatory threshold

\section{Introduction}

A high maximal oxygen uptake $\left(\mathrm{VO}_{2} \mathrm{max}\right)$ is an important prerequisite for endurance performance. However, $\mathrm{VO}_{2}$ max intensity can only be maintained for approximately 10-15 minutes $(2,6)$. Several endurance events last beyond 10-15 minutes and are performed at an intensity below the 
maximal oxygen uptake $(2,8,9,20)$. Studies in which the relationship between plasma lactate response, $\mathrm{VO}_{2} \max$, and endurance performance were investigated showed a higher correlation between endurance performance and lactate variables such as anaerobic threshold, than with $\mathrm{VO}_{2} \max (7,17,22)$.

Threshold indices are usually assessed in incremental exercise tests, often using the blood lactate response as a function of exercise intensity. A potential drawback of lactate measurements in incremental exercise tests is that it may take at least 3 minutes in an incremental exercise test to establish steady plasma lactate concentrations at a given exercise intensity $(3,10,14,16,18,19,24)$. This would imply that studies on lactate measurements in incremental exercise tests in which stages of less than 3 minutes were used, may have failed to assess the proper plasma lactate concentrations. The duration per stage before a steady state in plasma lactate concentration is reached appears to be related to the increment used. Stockhausen et al (23) proposed for cycling a duration of $3 \mathrm{~min}$ when using a 20 watt increment and a stage duration of $4 \mathrm{~min}$ when using a 30 watt increment. In a recent study Kuipers et al (14) showed that in incremental exercise tests on a cycle ergometer each stage should last at least 5 minutes before the blood lactate concentration levels off. However, with longer duration per stage peak values of heart rate and $\mathrm{VO}_{2}$ were significantly lower compared with 1 or 2.5 minutes per stage. It is unknown whether such differences in threshold variables and peak values also hold for incremental running protocols with different stage duration. In the study of Kuipers et al (14) it was also found that maximal lactate steady state (maxLASS) can be estimated by using the power output at which blood lactate was $4 \mathrm{mmol} / \mathrm{l}$ (P4) in an incremental protocol with 5 minute stages. For more precise assessment three 15 minutes stages at intensities P4 minus 25 watt, at P4, and P4 plus 25 watt were used. However, it has been reported that some physiological variables such as $\mathrm{VO}_{2}$ max are different between cycling and running. This may be true for the lactate response as well.

These questions led to the present study in which our first aim was to compare the response of some key physiological variables as well as their peak values in incremental running protocols in which the duration per stage was different ( $1 \mathrm{~min}, 3 \mathrm{~min}$, and 6 minutes). A second aim was to relate the various lactate and ventilatory thresholds obtained in the different protocols with the maximal lactate steady state. A third aim was to compare the blood lactate concentration at the maxLASS speed on the treadmill and on an outdoor track. Finally we intended to look for a relationship between actual athletic performances and some threshold indices. 


\section{Materials and methods}

\section{Subjects}

After approval from the local ethical committee 6 male and 4 female welltrained middle distance runners entered the study after written informed consent. All subjects trained at least 4 times per week for at least one hour per training session. Because of injuries two male subjects were unable to complete participation in the study. The mean age of the remaining 4 male and 4 female subjects was $33 \pm 12$ years (range 19-52), mean weight $68 \pm$ $8 \mathrm{~kg}$, mean height $183 \pm 9 \mathrm{~cm}$.

\section{Procedures}

The subjects refrained from strenuous exercise 24 hours before each exercise test. After the subjects reported to the lab, a venous catheter was inserted into a forearm vein. A stopcock was connected to the catheter, to enable collection of blood. The subject was connected to a heart rate monitor (Polar), and wore a face mask, connected to a portable metabolic system (Cosmed K4). Subsequently the subjects stepped on the treadmill (Medifit, The Netherlands) after which the test was started. A $1 \%$ inclined of the treadmill was maintained, in order to match energetic cost of outdoor running at the same speed (12).

\section{Exercise protocols}

The study took place in summer time, and all subjects had been in training for the oncoming season for at least three months. For studying the physiological response in an incremental running test with different duration per stage, 3 incremental running protocols were performed on a treadmill. The order of the first three protocols was random, and at least one week separated two consecutive tests.

Protocol A started with a warming up of 5 minutes at $8 \mathrm{~km} / \mathrm{hr}$ in women and $10 \mathrm{~km} / \mathrm{h}$ in men, where after every minute the exercise intensity was increased by $1 \mathrm{~km} / \mathrm{hr}$ until the subject was unable to continue.

Protocol B also started with a 5 min warming up at $8 \mathrm{~km} / \mathrm{hr}$ for women and $10 \mathrm{~km} / \mathrm{hr}$ for men, after which every 3 minutes the running speed was increased by $2 \mathrm{~km} / \mathrm{hr}$, and from a heart rate of $85 \%$ of maximal heart rate with $1 \mathrm{~km} / \mathrm{hr}$ every three minutes.

Protocol C was similar to protocol B with the only difference that every stage lasted 6 minutes.

If in protocols $B$ and $C$ a stage could not be completed to the full length the maximal running speed ( $V \max$ ) was calculated according to the equation:

\section{$V \max =V$ completed $+t / T^{*}$ speed increment}


in which Vcompleted is the running speed of the last stage that was completed, $t$ the number of seconds that the uncompleted running speed could be sustained, $T$ the number of seconds required to complete the stage, and speed increment is the speed load increment.

To assess maximal lactate steady state, a fourth protocol (D) was conducted, that consisted of 3 incremental workloads each lasting 15 minutes, preceded by a $5 \mathrm{~min}$ warm up at $50 \%$ of the previously assessed maximal running speed attained in the 6 minute per stage protocol. The three intensities of protocol $D$ were based on the running speed from protocol $C$ at which the blood lactate concentration was $4 \mathrm{mmol} / \mathrm{l}$ (V4). The first speed was $0.5 \mathrm{~km} / \mathrm{hr}$ below $\mathrm{V} 4$, the second at $\mathrm{V} 4$, and the third $0.5 \mathrm{~km} / \mathrm{hr}$ above V4. Maximal lactate steady state (maxLASS) as defined as a difference in blood lactate concentration of $<0.5 \mathrm{mmol} / \mathrm{l}$ between 10 and 15 minutes exercise at the same intensity.

To compare the blood lactate concentration at the speed of maxLASS measured on the treadmill and running outdoor, the subjects also ran 5 kilometer without interruption on a $\mathbf{4 0 0}$ meter tartan track at the maxLASS speed. In order to maintain a steady speed a supervisor on a bicycle remained in close proximity of the running athlete and clocked the split time every $\mathbf{2 0 0}$ meter, and gave feed back to the athletes. Immediately after completing the $5 \mathrm{~km}$ run, a venous blood sample was taken for lactate measurement.

\section{Variables}

Respiratory variables and heart rate were measured continuously, and displayed every 30 seconds on a portable computer, that was connected to the receiving unit of the $\mathrm{K} 4$ metabolic system.

From the respiratory variables the ventilatory threshold, based on $\mathrm{VE} / \mathrm{VO}_{2}$ was determined by the software provided with the Cosmed K4 measuring system using linear regression. The ventilatory threshold was defined as the running speed at which the $\mathrm{VE} / \mathrm{VCO}_{2}$ started to depart from linear increase. In addition a break point in the linear increase of the ventilation rate was searched for.

From the venous catheter a $2 \mathrm{ml}$ blood sample was drawn during the final $30 \mathrm{sec}$ of every stage, except for protocol A (increments every minute) in which no blood samples were taken, because of too short time available for blood sampling. From all samples the first $0.5 \mathrm{ml}$ of the sample was discarded because of dilution with saline. After sampling the catheter was flushed with saline. To obtain a blood sample the subject grasped the railing and stepped next to the running belt. Blood sampling required 15 to 20 seconds. Immediately after sampling the subject stepped back on the running belt and continued running. In protocol D (15 minute stages) for assessing maxLASS, blood was sampled every 5 minutes. The blood 
lactate concentration in whole blood was measured immediately after sampling (EML-105, Radiometer, Copenhagen, Denmark).

\section{Relationship between threshold values and running performance}

Every subject reported his or her personal best times attained within two months prior to the testing procedure. If no time of a certain distance was available the best time from the previous season was taken.

\section{Data handling and statistical analysis}

The data obtained during the last 30 seconds of the various protocols were used for analysis.

The data were analyzed with a SPSS software packet analyzed using a repeated measures ANOVA. Depending on the Mauchly's test of sphericity a uni- or multi-variate test was employed. Differences between the protocols were analyzed with a paired t-test. In case of univariate testing a Bonferroni correction was applied. Differences were considered statistically significant at $\mathrm{P}<0.05$.

\section{Results}

The mean peak running speed during the incremental protocols was inversely related to the duration of the stages (Table 1). The highest mean peak running speed was attained in protocol $A$ in which the load was increased by $1 \mathrm{~km} / \mathrm{hr}$ every minute (mean $18.3 \pm 1.9 \mathrm{~km} / \mathrm{hr}$ ), while the lowest peak power output was attained in the protocol with 6 minute stage $(15.1 \pm 1.5 \mathrm{~km} / \mathrm{hr} ; \mathrm{P}<0.01$ compared with the 1 -minute protocol).

\section{TABLE 1}

Maximal running velocity ( $V \max )$, peak $\mathrm{VO}_{2}$, peak heart rate (PeakHR), peak blood lactate concentration (PeakLa), the running speed at $4 \mathrm{mmol} / \mathrm{l}$ blood lactate (V4), and the running speed at breakpoint $\mathrm{VE} / \mathrm{VO}_{2}\left(\mathrm{Break} \mathrm{VE} / \mathrm{VO}_{2}\right)$ in incremental protocols with stage length of 1 minute (protocol A), 3 minutes (protocol B), and 6 minutes (protocol C).

\begin{tabular}{|ccccccc|}
\hline Protocol & $\begin{array}{c}\text { running speed } \\
(\mathrm{Km} / \mathrm{h})\end{array}$ & $\begin{array}{c}\mathrm{PeakVO}_{2} \\
(\mathrm{I} / \mathrm{min})\end{array}$ & $\begin{array}{c}\text { Peak HR } \\
(\text { beats } / \mathrm{min})\end{array}$ & $\begin{array}{c}\text { Peak Lactate } \\
(\mathrm{mmol} / \mathrm{l})\end{array}$ & $\begin{array}{c}\mathrm{V} 4 \mathrm{mmol} / \mathrm{I} \\
(\mathrm{km} / \mathrm{h})\end{array}$ & $\begin{array}{c}\text { Break VE/VO } \\
(\mathrm{km} / \mathrm{h})\end{array}$ \\
\hline $\mathrm{A}$ & $18.3 \pm 1.9$ & $4.48 \pm 1.13$ & $183 \pm 6$ & & & $14.0 \pm 1.8$ \\
$\mathrm{~B}$ & $17.1 \pm 1.6 \#$ & $4.46 \pm 1.04$ & $187 \pm 5$ & $11.0 \pm 2.5$ & $14.4 \pm 1.4$ & $13.3 \pm 1.6$ \\
$\mathrm{C}$ & $15.1 \pm 1.5 \# \#$ & $4.41 \pm 1.01$ & $189 \pm 5$ & $10.8 \pm 2.6$ & $12.9 \pm 1.5 \#$ & $13.4 \pm 1.4$ \\
\hline
\end{tabular}

Values are presented as mean $\pm S D$

$\# \mathrm{P}<0.05$ between $\mathrm{C}$ and $\mathrm{B} \quad \# \# \mathrm{P}<0.01 \mathrm{C}$ compared with $\mathrm{A}$ 
Mean peak $\mathrm{VO}_{2}$ (Table 1) was slightly higher in the protocol with 1-minute stages, compared with the protocol with 6 minutes states, although the difference did not reach statistical significance.

From table 1 it can be seen that the mean peak heart rate between the incremental protocols was not statistically significant.

The blood lactate response between the 3 and 6 minute protocols were different $(P<0.025)$, i.e. in all subjects the curve obtained from the 3 minutes per stage protocol was shifted to the right compared with the 6 minute protocol (Figure 1).

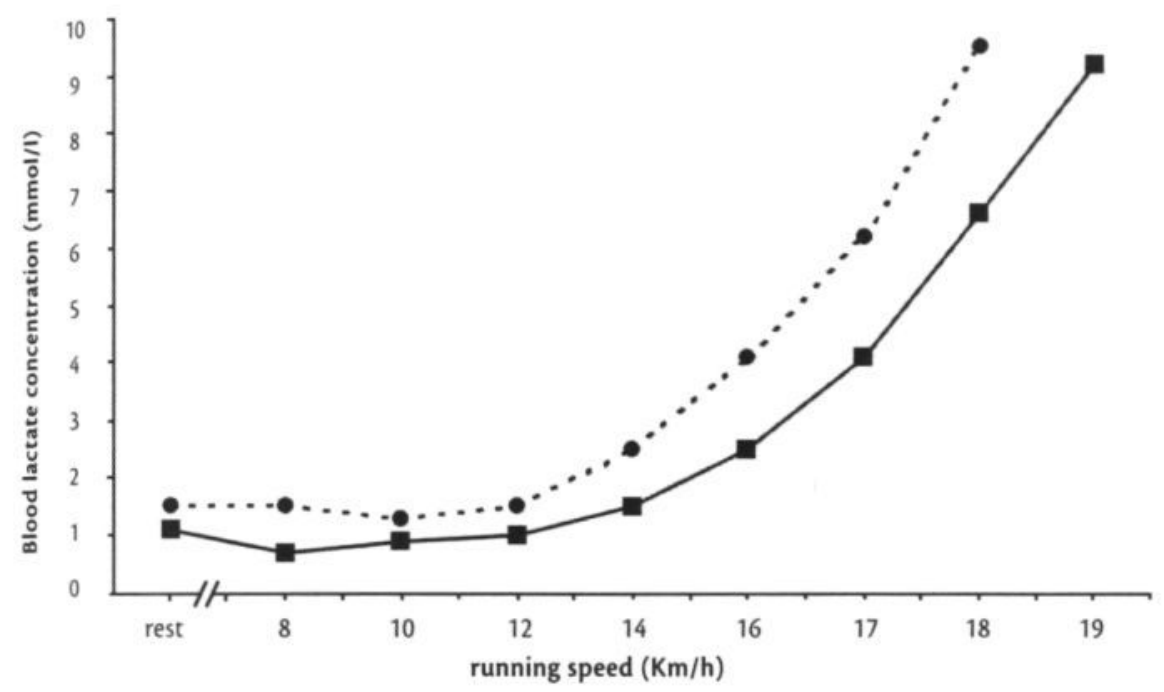

\section{FIGURE 1}

Example of blood lactate concentration of a male subject as function of power output in two different incremental exercise protocols with stages lasting 3 minutes (squares), and 6 minutes (circles).

The mean peak blood lactate concentration in the protocol with 3 minute stages was not different from the protocol with 6 minute stages. Since the $4 \mathrm{mmol} / \mathrm{l}$ intensity is often used as parameter for endurance capacity, the running speed at which the blood lactate concentration was $4 \mathrm{mmol} / \mathrm{I}$ (V4) was assessed in both protocols with 3 and 6 minute stages. V4 was significantly higher in the 3 minute per stage protocol $(14.4 \pm 1.4 \mathrm{~km} / \mathrm{hr}$ ) compared with the 6 minutes per stage protocol $(12.9 \pm 1.5 \mathrm{~km} / \mathrm{hr} ; \mathrm{P}<0.05)$. As can be seen from Table 1 the power output at which $\mathrm{VE} / \mathrm{VO}_{2}$ departed from a linear increase was $14.0 \pm 1.8 \mathrm{~km} / \mathrm{hr}$ in the 1 -minute per stage 
protocol, $13.3 \pm 1.6 \mathrm{~km} / \mathrm{hr}$ in the $3 \mathrm{~min}$ per stage protocol, and $13.4 \pm 1.4$ $\mathrm{km} / \mathrm{hr}$ in the 6 minute stage protocol $(P>0.05)$.

The running speed of the 15 minute protocol was based on the running speed at $4 \mathrm{mmol} / \mathrm{l}$ blood lactate concentration (V4) in the 6-minute per stage protocol. The running speed at $\mathrm{V} 4$ in the incremental 6 min protocol was $12.9 \pm 1.9 \mathrm{~km} / \mathrm{hr}$, whereas the running speed at which the maxLASS was reached in the 15 minute per stage protocol was $12.9 \pm 1.7 \mathrm{~km} / \mathrm{hr}(P>0.05)$. The mean blood lactate concentration reached at maxLASS was $6.03 \pm 1.47$ $\mathrm{mmol} / \mathrm{l}$ (range between individuals $3.8-7.2 \mathrm{mmol} / \mathrm{I}$ ). All subjects completed the 15 minutes at $\mathrm{V} 4$, and were able to start with a running speed of $V 4$ plus $0.5 \mathrm{~km} / \mathrm{hr}$, however 6 stopped prematurely because of fatigue. Blood lactate concentrations at $\mathrm{V} 4$ plus $0.5 \mathrm{~km} / \mathrm{hr}$ increased progressively (varying between individuals from 3.2 to $10.9 \mathrm{mmol} / \mathrm{l}$ ). Only 2 subjects were able to finish the 15 minutes with difficulty, and blood lactate levels raised to 8.5 and $10.2 \mathrm{mmol} / \mathrm{l}$ in these two subjects, respectively.

The mean blood lactate concentration after the $5 \mathrm{~km}$ run on the outdoor track was $6.32 \pm 2.12 \mathrm{mmol} / \mathrm{l}$ and was not different from the blood lactate concentration at the maxLASS speed during the 15 minute protocol. All subjects experienced the speed at maxLASS as strenuous, however, all indicated that this was below the competitive speed.

The mean running speed in the best competitive performance at different running distances was $15.0 \pm 1.2 \mathrm{~km} / \mathrm{hr}$ on the $5 \mathrm{~km}$ and $14.5 \pm 0.9 \mathrm{~km} / \mathrm{hr}$ on the $10 \mathrm{~km}$.

\section{Discussion}

A first aim of the present study was to investigate peak values of some physiological variables in an incremental running protocol in dependence of the duration of the stages. The present study demonstrates that in a protocol in which the running speed was increased by $1 \mathrm{~km} / \mathrm{hr}$ every minute, the maximal running speed is on the average $1.2 \mathrm{~km} / \mathrm{hr}$ higher compared to a protocol in which the running speed was increased every 3 minute, and on the average $3.2 \mathrm{~km} / \mathrm{hr}$ higher compared with a protocol in which the running speed was increased every 6 minutes. These findings are in line with those found in a similar study using a cycle ergometer (14).The higher maximal running speed in a protocol with a short time per stage can be explained by a greater contribution of anaerobic power at the later load steps, due to the delayed lactate accumulation that is associated with local muscle fatigue. Since maximal running speed can be used as a parameter for endurance capacity $(15,21)$, the different maximal running speed in the protocols with different duration per stage have to be taken into account when reporting maximal running speeds, and when 
comparing running speeds attained in incremental protocols with different duration per stage.

The peak $\mathrm{VO}_{2}$ was not different between the tests with different stage duration, which is not in line with the findings on a cycle ergometer, where $\mathrm{VO}_{2}$ peak was lower when the duration per stage was longer (14). The difference between an incremental test on a treadmill and on a cycle ergometer may be attributable to the larger muscle mass involved in running, making local muscle fatigue less important as a limiting factor compared to cycling. This implies that for measuring $\mathrm{VO}_{2} \max$ in an incremental protocol on a treadmill the duration per stage is of less importance and may vary from 1 to 6 minutes.

The data of the present study demonstrate that for measuring blood lactate concentrations belonging to a given exercise intensity, a longer duration per stage is required. The results of the present study show that when the duration per stage in incremental protocols is shorter than approximately 6 minutes, blood lactate concentrations lag behind the corresponding exercise intensity. This is in line with the findings of Foxdall et al (10) who compared the blood lactate response in incremental treadmill protocols with 4, 6 and 8 minute stages, and Kuipers et al (10) who compared stage durations of 3 and 5 minutes on a cycle ergometer. This would also imply that incremental exercise protocols for measuring blood lactate parameters such as the $4 \mathrm{mmol} / \mathrm{l}$ intensity, load steps less than approximately 5 minutes yield non-steady state values for blood lactate. The exercise intensity at which blood lactate concentration equals $4 \mathrm{mmol} / \mathrm{l}$ is often used as a parameter for endurance capacity $(1,7)$. When looking at the incremental protocol with different duration per stage at the running velocity at which blood lactate concentration is $4 \mathrm{mmol} / \mathrm{I}$ (V4), we found that V4 was on the average $1.5 \mathrm{~km} / \mathrm{hr}$ lower in the protocol with 6 minutes per stage, compared with the protocol with 3 minutes per stage. This reflects the insufficient time for lactate equilibration in the incremental protocol with 3 minutes stages. In the present study maximal lactate steady state (maxLASS) speed was estimated from the running speed at $\mathrm{V} 4$ in the incremental running test with 6 minutes stages, and measured by a second incremental protocol with 15 minutes per stage on a separate day. The speeds of the 15 minutes stages were $\mathrm{V} 4$ minus $0.5 \mathrm{~km} / \mathrm{hr}, \mathrm{V} 4$, and $\mathrm{V} 4$ plus $0.5 \mathrm{~km} / \mathrm{hr}$. The mean running speed at $\mathrm{V} 4$ in the incremental protocol with 6 minute stages was not different from the mean velocity at maxLASS. However, the blood lactate levels in the 15 minute protocol were significantly higher than the $4 \mathrm{mmol} / \mathrm{l}$ in the 6 minute protocol. These results lead to the conclusion that the speed at maxLASS can be assessed just by doing an incremental protocol with 6 minute stages. However, for assessing the highest blood lactate levels at which a steady state can be maintained, exercise of longer 
duration is required. This supports the finding that for attaining a true steady state in blood lactate concentration a duration of 6 minutes is still insufficient. The 15 minutes duration at a given exercise intensity for assessing maximal lactate steady state is the same as used by Kuipers et al (14) in a cycle ergometer test, but shorter than used by other investigators who used 20-30 minutes of continuous exercise $(4,13)$. Jones \& Doust proposed to adopt 30 minutes constant exercise for assessing maxLASS in running. However, their results show that when no steady state is reached in approximately 10 minutes the exercise is not at maxLASS. Jenkins and Quigley (11) also observed that blood lactate levels remained stable between 10 and 30 minutes of continuous exercise. In a pilot study before the present investigation was started, in 5 subjects the blood lactate response during 30 minutes exercise per stages was studied. It was found that when no steady state in blood lactate concentration was attained between 10 and 15 minutes, no steady state was attained at all. The validity of 15 minute duration for assessing maxLASS was supported by the outdoor $5 \mathrm{~km}$ run at the maxLASS velocity that lasted 20-25 minutes. The longer duration elicited the same blood lactate concentration as the 15 minute treadmill run in the laboratory. Therefore, maxLASS speed can be approximated by $\mathrm{V} 4$ obtained from an incremental protocol with 6 minute stages, whereas the accurate assessment of lactate levels require a protocol consisting of 15 minute stages. The mean maximal blood lactate concentration at which a steady state could be maintained was $6.02 \mathrm{mmol} / \mathrm{l}$ (range 3.8-7.2 mmol/l). The considerable range reflects the possible different response between individuals.

Ventilatory threshold is also used as parameter for endurance capacity. In the present study ventilatory threshold was defined as the power output at which $\mathrm{VE} / \mathrm{VO}_{2}$ started to increase non-linearly. The results of the present study show that the running speed at which the non-linear increase in $\mathrm{VE} / \mathrm{VO}_{2}$ started, was not different between stage durations of 1 to 6 minutes (Table 1), which is in line with the findings of Zhang et al (25). This suggests that in the experiments conducted ventilatory response is not directly related to blood lactate response. In the present study is was also searched for possible threshold behaviour in breathing frequency. Using regression analysis no such breakpoint in ventilation rate consistent with lactate indices was found. This is in line with the findings of Jones and Doust (13) and indicates that respiratory rate cannot be used as threshold variable.

One of the aims was to study the relationship between maxLASS exercise intensity and other threshold variables, and endurance performance. The average running speed at $5 \mathrm{~km}$ was $15.0 \mathrm{~km} / \mathrm{hr}$ which is significantly higher than the running speed at maxLASS and $\mathrm{V} 4$ in the incremental test. The average running speed in a $10 \mathrm{~km}$ competion was $14.5 \mathrm{~km} / \mathrm{hr}$. A point that 
has received little attention is the observation that there is no such thing as one critical speed at which endurance performances are done. When the average speeds of world records at different distances in certain disciplines are compared it appears that the average speed declines with increasing distance. This indicates that there is not one single critical speed, but it decreases with increasing distance. This is supported by the results from the study of Jones \& Doust (13) who studied the relationship between $8 \mathrm{~km}$ running performance and lactate parameters, and Bentley et al (5) who studied the relationship between lactate variables and 20 minute and 90 minute time trials. Foster et al (9) studied lactate levels during a simulated time trial over $5 \mathrm{~km}$ in cyclists, and observed that plasma lactate levels increased throughout the trial. From the present study it is concluded that the running speed at 5 and $10 \mathrm{~km}$ cannot be predicted from the results of running tests in the laboratory. Therefore, it can be concluded that lactate threshold variables may be helpful for assessing endurance characteristics in athletes, however have limited value for predicting endurance performances.

In conclusion, peak $\mathrm{VO}_{2}$ and peak heart rates can be obtained in incremental protocols with increments varying from 1 to 6 minutes. When steady blood lactate levels are required the duration per stage should be at least 6 minutes, preferably 8-10 minutes. The maxLASS running velocity can be estimated from running speed at $4 \mathrm{mmol} / \mathrm{l}(\mathrm{V} 4)$ in an incremental protocol with 6 minutes per stage, but must be measured by 3 velocities of 15 minutes duration at V4 minus $0.5 \mathrm{~km} / \mathrm{hr}$, at $\mathrm{V} 4$, and $0.5 \mathrm{~km} / \mathrm{hr}$ above $\mathrm{V} 4$.

\section{Acknowledgement}

This work was supported by grants from the Netherlands Olympic Committee and Netherlands Sports Federation (NOC*NSF). 


\section{References}

1. Anderson G.S., E.C. Rhodes. A review of blood lactate and ventilatory methods of detecting transition thresholds. Sports Med 8: 43-55. 1989.

2. Astrand P.O. Endurance in sports. In: Endurance sports, edited by S. Astrand. London: Blackwell Science, 2000, p. 9-15.

3. Aunola S., H. Rusko. Does anaerobic threshold correlate with maximal lactate steady-state? J Sports Sci 10: 309-323. 1992.

4. Beneke R., S.P. von Duvillard. Determination of maximal lactate steady state response in selected sports events. Med Sci Sports Exerc 28: 241-246. 1996.

5. Bentley D.J., L.R. McNaughton, D. Thompson, V.E. Vleck, A.M. Batterham. Peak power output, the lactate threshold, and time trial performance in cyclists. Med Sci Sports Exerc 33: 2077-2081. 2001.

6. Billat L.V., J.P. Koralsztein. Significance of the velocity at VO2max and time to exhaustion at this velocity. Sports Med 22: 90-108. 1996.

7. Bishop D., D.G. Jenkins, L.T. Mackinnon. The relationship between plasma lactate parameters, Wpeak and 1-h cycling performance in women. Med Sci Sports Exerc 30: 1270-1275. 1998.

8. Costill D.L., H. Thomason, E. Roberts. Fractional utilization of the aerobic capacity during distance running. Med Sci Sports 5: 248-252. 1973.

9. Foster C., M.A. Green, A.C. Snyder, N.N. Thompson. Physiological responses during simulated competition. Med Sci Sports Exerc 25: 877-882. 1993.

10. Foxdal P., A. Sjodin, B. Sjodin. Comparison of blood lactate concentrations obtained during incremental and constant intensity exercise. Int / Sports Med 17: 360-365. 1996.

11. Jenkins D.G., B.M. Quigley. Blood lactate in trained cyclists during cycle ergometry at critical power. Eur J Appl Physiol Occup Physiol 61: 278-283 1990.

12. Jones A.M., J.H. Doust. A $1 \%$ treadmill grade most accurately reflects the energetic cost of outdoor running. I Sports Sci 14: 321-327. 1996.

13. Jones A.M., J.H. Doust. The validity of the lactate minimum test for determination of the maximal lactate steady state. Med Sci Sports Exerc 30: 1304-1313. 1998.

14. Kuipers H., G.J.W.M. Rietjens, F.T. Verstappen, W. Vanruiten, T. Koelewijn. Physiological variables and lactate indices in incremantal exercise tests with different stage duration. Br J Sport med submitted 2002.

15. Lucia A., J. Pardo, A. Durantez, J. Hoyos, J.L. Chicharro. Physiological differences between professional and elite road cyclists. Int J Sports Med 19: 342-348. 1998.

16. MacDougall D., D. Sale. Continuous vs. interval training: a review for the athlete and the coach. Can-J-Appl-Sport-Sci 6: 93-97 1981.

17. Maffulli N., G. Capasso, A. Lancia. Anaerobic threshold and performance in middle and long distance running. I Sports Med Phys Fitness 31: 332-338. 1991.

18. McLellan T.M. Ventilatory and plasma lactate response with different exercise protocols: a comparison of methods. Int J Sports Med 6: 30-35. 1985.

19. Moreau K.L., M.H. Whaley, J.H. Ross, L.A. Kaminsky. The effects of blood lactate concentration on perception of effort during graded and steady state treadmill exercise. Int J Sports Med 20: 269-274. 1999.

20. Morgan D.W., J.T. Daniels. Relationship between VO2max and the aerobic demand of running in elite distance runners. Int J Sports Med 15: 426-429. 1994. 
21. Mujika I., S. Padilla. Physiological and performance characteristics of male professional road cyclists. Sports Med 31: 479-487 2001.

22. Nicholson R.M., G.G. Sleivert. Indices of lactate threshold and their relationship with 10-km running velocity. Med Sci Sports Exerc 33: 339-342. 2001.

23. Stockhausen W., D. Grathwohl, C. Burklin, P. Spranz, J. Keul. Stage duration and increase of work load in incremental testing on a cycle ergometer. Eur J Appl Physiol Occup Physiol 76: 295-301 1997.

24. Yoshida T. Effect of exercise duration during incremental exercise on the determination of anaerobic threshold and the onset of blood lactate accumulation. Eur J Appl Physiol Occup Physiol 53: 196-199 1984.

25. Zhang Y.Y., M.C. Johnson, 2nd, N. Chow, K. Wasserman. Effect of exercise testing protocol on parameters of aerobic function. Med Sci Sports Exerc 23: 625-630. 1991. 



\section{CHAPTER 7}

\section{Ciêu}

A reduction in training volume and intensity during 21-days does not impair performance in cyclists

\section{This Chapter has been published as:}

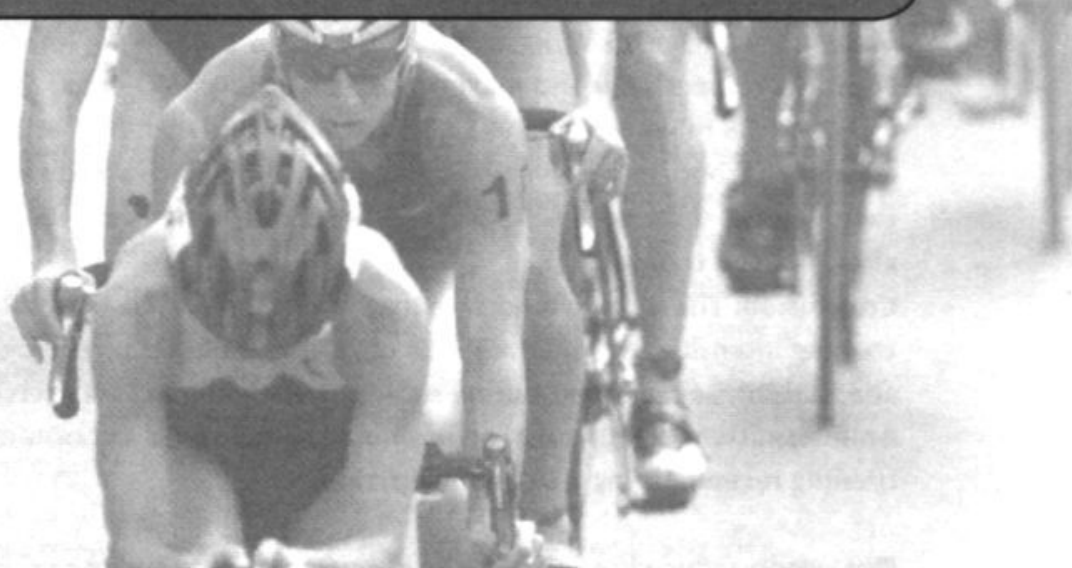

A reduction in training volume and intensity during 21-days does not impair performance in cyclists of

GJWM Rietjens', H Kuipers', WHM Saris', HA Keizer'

'Department of Movement Sciences, Maastricht University, Maastricht, The Netherlands

2 Department of Human Biology, Maastricht University, Maastricht, The Netherlands a) 19

\section{British Journal of Sports Medicine}




\section{Abstract}

Objectives: The first aim of this study was to investigate the effects of reduced training on physical condition and performance in well-trained cyclists. The second aim was to study whether an intermittent exercise program would maintain physiological training adaptations better than a continuous exercise program during a period of reduced training.

Methods: Twelve male cyclists participated in a 21-day training program and were divided into two training groups. One group ( $25.3 \pm 7 \mathrm{yr}$ (mean $\pm \mathrm{SD}$ ), $73.3 \pm 5.7 \mathrm{~kg}, \mathrm{VO}_{2} \max 58.6 \pm 4.5 \mathrm{ml} \cdot \mathrm{kg}^{-1} \cdot \mathrm{min}^{-1}$ ) underwent a continuous endurance exercise training program (CT) whereas the second group $\left(22.8 \pm 3.5 \mathrm{yr}, 74.1 \pm 7.0 \mathrm{~kg}, \mathrm{VO}_{2} \max 59.7 \pm 6.7 \mathrm{ml} \cdot \mathrm{kg}^{-1} \cdot \mathrm{min}^{-1}\right)$ followed an intermittent endurance exercise training program (IT). During this reduced training period both groups trained for two hours a day, three days per week.

Results: Neither group showed changes in maximal workload (Wmax) ( $4.6 \pm 0.5$ vs. $4.8 \pm 0.5 \mathrm{~W} / \mathrm{kg}$ and $4.6 \pm 0.5$ vs. $4.7 \pm 0.6 \mathrm{~W} / \mathrm{kg}$ for the CT and IT group, respectively) and maximal oxygen uptake $\left(\mathrm{VO}_{2} \mathrm{max}\right)(58.6 \pm 4.5$ vs. $60.1 \pm 5.8 \mathrm{ml} \cdot \mathrm{kg}^{-1} \cdot \mathrm{min}^{-1}$ and $59.7 \pm 6.7$ vs. $58.8 \pm 7.5 \mathrm{ml} \cdot \mathrm{kg}^{-1} \cdot \mathrm{min}^{-1}$ for the $\mathrm{CT}$ and IT group, respectively). During the sub-maximal steady state exercise test, substrate utilization and heart rate remained unchanged after reduced training.

Conclusion: These results indicate that well-trained cyclists who lower training intensity and volume for $\mathbf{2 1}$ days, can maintain physiological adaptations, as measured during sub-maximal and maximal exercise. An intermittent training regime has no advantage over a continuous training regime during a detraining period.

Key words: reduced training, cycling, performance, substrate utilization.

\section{Introduction}

In order to improve endurance performance, some athletes reduce the training load for 6 to 21 days before a major event $(1,19)$, a training technique known as tapering. Planned tapering consists generally of high intensity exercise, however with low volume. After a planned period of tapering, increased performance has been demonstrated in several studies with swimmers $(1,8,15,20,23)$, runners $(11,13,14,22)$ and cyclists $(17$, 18 ). On the basis of these studies it has been concluded that a low volume but high intensity taper program may enhance performance $(5,6,17,22)$. Such a low volume high intensity taper is usually planned and included in the training program. However, athletes are sometimes forced to lower 
both the training volume and intensity for several weeks due to illness, injury or other reasons. Although scientific studies are lacking, athletes and coaches are concerned that such low intensity exercise may result in a decrement in physical conditioning and performance, as demonstrated in some detraining studies $(6,7,9,11)$. However, these studies reduced training volume but maintained a high intensity. Therefore, the first aim of this study was to investigate whether cyclists who reduce their training volume by $50 \%$ and also lower intensity for 21 days can maintain physical performance capacity. The second aim was to investigate whether an intermittent training program has an advantage over continuous exercise with a reduction of both training volume and intensity. Since endurance training and detraining may be reflected in changes in relative fat oxidation during exercise, possible changes in the rate of fat oxidation after a period of reduced training, were also studied.

\section{Materials and Methods}

\section{Subjects}

Twelve well-trained male cyclists aged $24 \pm 5$ (mean \pm SD) years participated in this study. All testing and training procedures were approved by the Medical Ethical Committee of the Maastricht University, and an informed consent form was signed by all participants. Physical and training characteristics of the subjects at the start of the study are presented in Table 1.

\section{TABLE 1}

Physical and training characteristics at the start of the study (maxtest 1)

\begin{tabular}{|c|c|c|c|c|c|}
\hline \multirow[b]{2}{*}{ Age (yr) } & \multicolumn{2}{|r|}{ CT } & \multicolumn{3}{|c|}{ IT } \\
\hline & 25.3 & \pm 7.0 & 22.8 & \pm & 3.5 \\
\hline Height $(\mathrm{cm})$ & 181.8 & \pm 6.1 & 187.5 & \pm & 5.3 \\
\hline Weight (kg) & 73.3 & \pm 5.7 & 74.1 & \pm & 7.0 \\
\hline Percent Fat (\%) & 12.4 & \pm 2.5 & 12.8 & \pm & 2.7 \\
\hline Fat-free mass $(\mathrm{kg})$ & 64.1 & \pm 4.6 & 64.6 & \pm & 5.4 \\
\hline Maximal Power Output (Watt/kg) & 4.5 & \pm 0.46 & 4.5 & \pm & 0.54 \\
\hline VO2 max. $\left(\mathrm{ml}^{\prime} \mathrm{kg}^{-1} \cdot \min ^{-1}\right)$ & 58.4 & \pm 5.5 & 59.2 & \pm & 4.8 \\
\hline Lactate treshold $\left(\% \mathrm{VO}_{2} \max \right)$ & 68.7 & \pm 3.0 & 67.6 & \pm & 4.9 \\
\hline Years of training & 6.0 & \pm 2.1 & 7.5 & \pm & 4.2 \\
\hline Training volume (hrs/wk)\# & 15.6 & \pm 1.1 & 16.1 & \pm & 1.5 \\
\hline
\end{tabular}

(values are mean $\pm S D$ ), $n=6$ cyclists per group). \#training volume over the last two months prior to the start of the study. $\mathrm{CT}=$ Continuous Training program and $\mathrm{IT}=$ Intermittent Training program. 


\section{General Design}

To create a realistic reduced training scenario, twelve well-trained nonelite cyclists were recruited, who were fully prepared for a competition season.

The training status of every subject over the previous two months and the training history was obtained by a questionnaire, training log and a personal interview. We considered the subjects well trained if they trained at least five years, 2 hours a day four to five times a week. Only subjects who met these criteria were included in the study. Two days before the start of a seven-day pre-training period, each subject performed a maximal bicycle ergometer ride (maxtest 1 ) to determine maximal workload (Wmax) and maximal oxygen uptake. This test was repeated after the pre-intervention training week. After this maximal workload test the subjects were assigned to either the intermittent exercise training group (IT group, $n=6$ ) or the continuous exercise training group (CT group, $n=6$ ). Subjects were matched for weekly training volume (hrs/week), training history (years of training), age and maximal oxygen uptake $\left(\mathrm{VO}_{2}\right.$ max $)$ in an attempt to obtain comparable groups.

In order to familiarize subjects with the laboratory procedures and to present an added training stimulus, all subjects underwent a seven-day training period prior to the 21-day experimental period. This seven day training period consisted of three hours training a day at an intensity of $80 \% \mathrm{VO}_{2}$ max. Maximal workload tests were undertaken immediately prior to the 21-day reduced training period (maxtest 2 ) and at the end of the period (maxtest 3).

During the study, training volume was reduced to $50 \%$ of each individual's average normal weekly training volume (hours/week).

On days one, seven, fourteen and twenty-one the subjects underwent a 90-minute steady state indirect calorimetry cycle test (CT-90) (Excalibur, Lode, Groningen, The Netherlands) at their individual lactate threshold in order to study substrate utilization during exercise. Gas exchange was measured, at 8, 33, 58 and 83 minutes after the onset of exercise whilst the subjects were temporarily connected to a metabolic system (Oxycon B, E. Jaeger $\mathrm{GmbH}$, Höchberg, Germany) for seven minutes per measurement. The subjects completed their entire training program (pre training and 21-day reduced training program) in the laboratory under supervision. Training sessions were performed on calibrated electrically braked cycle ergometers (Excalibur, Lode, Groningen, The Netherlands or Recordtrainer, Avatronic Systeme, Leipzig, Germany). 


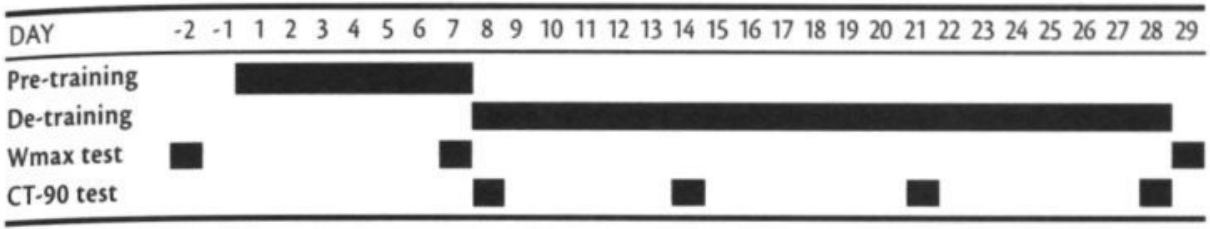

\section{FIGURE 1}

Schematic overview of the study design

\section{Test procedures}

All tests were performed between 08.00 and 10.00 A.M. after an overnight fast. The subjects were requested to maintain their normal habitual diet and abstain from training the day before a laboratory tests.

Maximal workload test To determine sub-maximal and maximal metabolic and cardiorespiratory capacity, an incremental exercise test was performed on an electrically braked cycle ergometer (Excalibur, Lode, Groningen, The Netherlands). The initial workload of the maximal workload test was set at $95 \mathrm{~W}$ and thereafter increased by $35 \mathrm{~W}$ every five minutes until volitional exhaustion. The $W \max$ was defined as the last completed stage (in Watt) plus the fraction of time spent in the final work rate multiplied by $35 \mathrm{~W}$ (16). During the test gas exchange (Oxycon B, E. Jaeger $\mathrm{GmbH}$, Höchberg, Germany) and heart rate (Sporttester, Polar, Finland) were measured continuously. Before each test, the respiratory gas analyzer was calibrated using certified calibration gas (Carbon dioxide $3.994 \pm 0.040$ vol. \%, Oxygen $15,99 \pm 0.16$ vol. \%, Nitrogen 80 vol. \%) and flow-volume accuracy was checked using a 3-liter syringe (Hans Rudolf Inc, Kansas City USA). In addition, venous blood samples for lactate deter-mination were collected during the maximal workload tests. Blood lactate samples were collected immediately prior to the start of the maximal workload test, during the last 30 seconds of every stage and immediately at the end of the test. Blood samples $(1 \mathrm{ml})$ were collected in heparinized tubes, centrifuged and the plasma was frozen at $-20^{\circ} \mathrm{C}$ until analyzed for lactate on a semi-automatic analyzer (Cobas FARA, La Roche, Basel, Switzerland). Lactate values were used for estimating subjects training intensity and to evaluate possible training effects in both groups.

Indirect calorimetry Because one of the effects of endurance training is a shift from carbohydrate to fat metabolism, reduction of the training may yield the opposite. For this reason substrate utilization during exercise was measured. To study substrate utilization during exercise, indirect calorimetry was performed during a 90-minutes steady state cycle test (CT-90). The initial exercise intensity during this test was set at the subjects individual lactate threshold (4) minus $5 \%$ obtained during the second 
maximal workload test (maxtest 2). Subjects were instructed to maintain the cadance between $85-95$ rpm during all four tests. Gas exchange measurements were taken at $8,33,58$ and 83 minutes after the onset of exercise. Each measurement lasted seven minutes. Expired gases were auto-analyzed and averages calculated over eight breaths (Oxycon B, E. Jaeger $\mathrm{GmbH}$, Höchberg, Germany). Before each test, gas analyzers of the Oxycon Beta were calibrated.

From respiratory $\mathrm{VO}_{2}$ and $\mathrm{VCO}_{2}$ measurements, carbohydrate and fat oxidation rates of each seven minutes period were calculated, by using the non-protein respiratory ratio (21).

\section{Fat oxidation $\quad=\quad 1.695 \mathrm{VO}_{2}-1.701 \mathrm{VCO}_{2}$ Carbohydrate oxidation $=4.585 \mathrm{VCO}_{2} \cdot 3.226 \mathrm{VO}_{2}$}

with $\mathrm{VO}_{2}$ and $\mathrm{VCO}_{2}$ in $1 \cdot \mathrm{min}^{-1}$ and oxidation rates in $\mathrm{g} \cdot \mathrm{min}^{-1}$. The average amount of fat oxidation of these four measurements was used to examine changes in fat oxidation during the reduced training period. Heart rate was measured continuously (Sporttester, Polar, Finland) during the CT-90. The CT-90 tests were part of the reduced training program.

\section{Pre training program}

Prior to the reduced training period, the subjects underwent a seven-day controlled endurance-training program. In this seven day pre-intervention period subjects had to train three hours a day at an intensity of approximately $80 \%$ of the individual maximal workload obtained in the first maximal workload test (maxtest 1 ).

\section{Reduced training program}

During the 21-day reduced training program, training volume and frequency were reduced by $50 \%$ and $20 \%$, respectively. The program consisted of training for two hours a day on 3 days each week (CT-90 not included). To define the exercise intensity during this period, the lactate curves obtained during maxtest 2 were used for determination of the lactate threshold according to Farrel et al. (4). The exercise intensity for the CT group was equal to the lactate threshold minus $5 \%$. The average exercise intensity in the $\mathrm{CT}$ group corresponded to $68 \% \mathrm{VO}_{2}$ max. For the subjects in the IT group the individual lactate threshold point minus $5 \%$ was calculated as well. Subjects performed an alternated training program at an exercise intensity of 10 minutes at $15 \%$ below and 10 minutes at $15 \%$ above this point. The average exercise intensities were $53 \%$ and $83 \% \mathrm{VO}_{2}$ max respectively. For both groups the average work output per training session was equal. 


\section{Statistical analyses}

The results are presented as mean $\pm S D$. The effect of training reduction on maximal and sub-maximal parameters within each training group was analyzed by a repeated-measures two-way analysis of variance. When a significant difference was observed, a post-hoc Scheffé F-test was performed to determine the location of the significant differences. Significant differences between the two training groups for performance was determined using an unpaired t-test. A P value of less than 0.05 was regarded as significant.

\section{Results}

All subjects completed the whole study and data from all subjects were included in the analyses. There were no significant differences between selected characteristics among the subjects (Table 1).

No significant differences were found within or between the groups for Wmax and $\mathrm{VO}_{2}$ max obtained in the three maximal workload tests.

The reduction in training volume and intensity had no effect on submaximal oxygen uptake $(P<0.43)$ and heart rate $(P<0.27)$ within or between the two groups (Table 2).

\section{TABLE 2}

Overview of maximal and sub maximal data obtained before (maxtest 2 ) and after (maxtest 3) the taper period.

\begin{tabular}{|c|c|c|c|c|}
\hline & \multicolumn{2}{|c|}{ Continuous training } & \multicolumn{2}{|c|}{ Intermittend training } \\
\hline & $\begin{array}{c}\text { pre-intervention } \\
\text { maxtest } 2\end{array}$ & $\begin{array}{c}\text { post-intervention } \\
\text { maxtest } 3\end{array}$ & $\begin{array}{l}\text { pre-intervention } \\
\text { maxtest } 2\end{array}$ & $\begin{array}{c}\text { post-intervention } \\
\text { maxtest } 3\end{array}$ \\
\hline at 165 Watt & & & & \\
\hline heart rate (beats/min) & $133 \pm 11$ & $133 \pm 11$ & $134 \pm 16$ & $136 \pm 15$ \\
\hline Lactate $(\mathrm{mmol} \cdot \mathrm{l})$ & $1.4 \pm 0.4$ & $1.4 \pm 0.3$ & $1.7 \pm 0.6$ & $1.2 \pm 0.2$ \\
\hline Oxygen uptake $\left(\mathrm{ml} \cdot \mathrm{kg}^{\prime} \cdot \mathrm{min}^{\prime}\right)$ & $29.9 \pm 1.4$ & $27.5 \pm 3.7$ & $30.6 \pm 3.8$ & $29.5 \pm 4.6$ \\
\hline at 270 Watt & & & & \\
\hline heart rate (beats/min) & $172 \pm 8$ & $169 \pm 11$ & $169 \pm 14$ & $166 \pm 21$ \\
\hline Lactate $(\mathrm{mmol} \cdot \mathrm{l})$ & $5.0 \pm 0.4$ & $3.9 \pm 0.3$ & $4.9 \pm 0.3$ & $4.0 \pm 0.9$ \\
\hline Oxygen uptake $\left(\mathrm{ml}^{\prime} \mathrm{kg}^{\prime} \cdot \mathrm{min}^{-1}\right)$ & $47.9 \pm 7.4$ & $47.6 \pm 8$ & $47.5 \pm 3.8$ & $47.4 \pm 5.3$ \\
\hline Maximal & & & & \\
\hline heart rate (beats/min) & $192 \pm 6$ & $193 \pm 5$ & $196 \pm 3$ & $195 \pm 4$ \\
\hline Power output $(\mathrm{W} \cdot \mathrm{kg})$ & $4.6 \pm 0.5$ & $4.8 \pm 0.5$ & $4.6 \pm 0.5$ & $4.7 \pm 0.6$ \\
\hline Oxygen uptake $\left(\mathrm{ml} \cdot \mathrm{kg}^{\prime} \cdot \min { }^{\prime}\right)$ & $58.6 \pm 4.5$ & $60.1 \pm 5.8$ & $59.7 \pm 6.7$ & $58.8 \pm 7.5$ \\
\hline
\end{tabular}

Values are expressed as mean \pm SD 
Table 3 displays the data obtained during the CT-90 test. Neither group showed a difference in the rate of fat oxidation after 7,14 or 21 days of training reduction. In addition, no changes in mean heart rates were found during the CT-90.

\section{TABLE 3}

Heart frequency and fat oxidation rate before (day 0 ) during (day 7 and day 14 ) and at the end (day 21) of the reduced training period

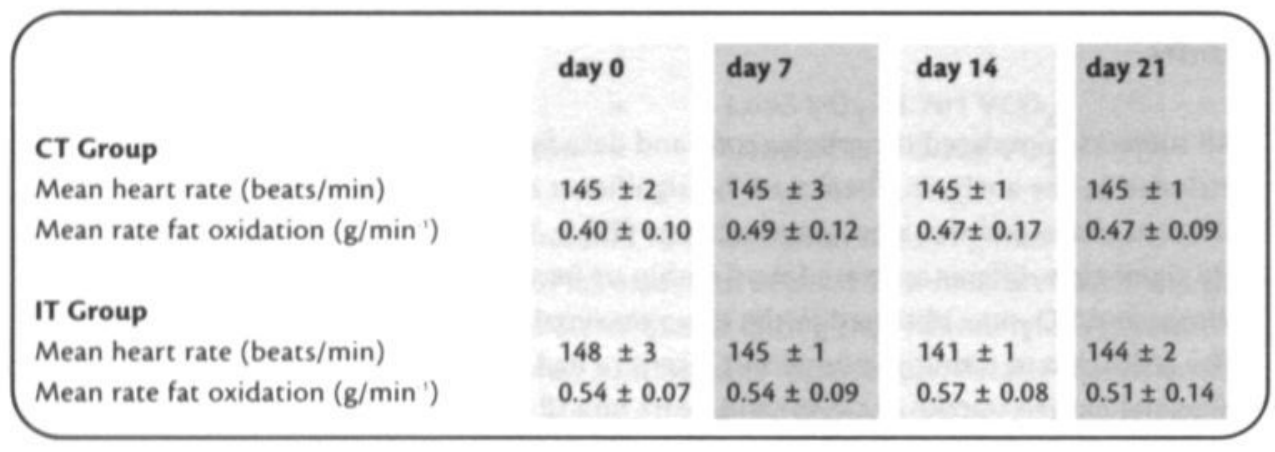

Values are expressed as mean \pm SD

\section{Discussion}

The present study examined the effects of two different training programs in which both the volume and intensity were reduced for 21-days. To ensure a similar training state in the athletes prior to the intervention, a seven-day high intensity training program was executed. The results show that the high intensity pre-training period for seven days did not affect the mean maximal workload and mean $\mathrm{VO}_{2} \max$. Therefore, it is to be expected that a reduction of the weekly training intensity to maximal $68 \%$ and $83 \% \mathrm{VO}_{2}$ max for the $\mathrm{CT}$ group and IT group respectively and a $50 \%$ and $20 \%$ reduction in volume and training frequency respectively might induce detraining effects (6). However, it was found that the mean maximal power output ( $W$ max) and the mean maximal oxygen uptake $\left(\mathrm{VO}_{2} \mathrm{max}\right)$ in the incremental cycle test did not change over the 21-day period with reduced training. This is a significant finding of this study since in previous studies that have examined the effects of reduced training volume in athletes, and reported that $\mathrm{Wmax}_{\operatorname{man}} \mathrm{VO}_{2}$ max were maintained for 10 to 28 days in trained endurance athletes $(7,10,11,14$, $15,18,20,23)$, the training intensity was maintained or even increased. An important finding of the present study is that $\mathrm{Wmax}$ and $\mathrm{VO}_{2} \max$ can 
be maintained in well trained cyclists for a period of three weeks, by reducing both volume and intensity $<70 \% \mathrm{VO}_{2}$ max. This contradicts with findings of Hickson et al. who concluded that a high training intensity (70-100\% of the normal training load) is an essential requirement for maintaining physiological adaptations gained with endurance training (6). Changes in performance capacity may be reflected in changes in the physiological response of heart rate and respiratory expiration ratio (RER) during exercise $(2,3,12)$. The results in our study do not support these findings, as there was no significant $(P<0.24)$ change in sub-maximal and maximal heart rate during the incremental cycle test or the CT-90 test. Also no changes in RER during sub-maximal exercise were found in the present study. An increase in RER during sub-maximal and maximal exercise has been shown after periods of reduced training and detraining, indicating a shift towards an increased reliance on carbohydrate during exercise $(3,11,14)$. In our study, based on RER values no differences in substrate utilization and rate of fat oxidation were found in either of the two training protocols throughout the 21 days of reduced training. This indicates that a $50 \%$ reduction in weekly training volume, with a reduction in training intensity for three weeks does not necessarily affect performance characteristics in cyclists. One of the aims of the present study was to investigate whether intermittent exercise had different effects on performance characteristics compared to continuous exercise. As the effect on performance was not different between the two exercise regimes, it can be concluded that an intermittent training program had no advantage over a continuous training program, on performance characteristics.

To summarize, this study demonstrates that well trained cyclists, who reduced training volume, training frequency and training intensity, can maintain their sub-maximal and maximal performance level for a period of at least 21 days. In a reduced training period intermittent exercise has no advantage over continuous exercise. These findings may have practical relevance, especially for those athletes who are forced to cut down the training intensity. 


\section{Acknowledgement}

This work was supported by grants from the Netherlands Olympic Committee and Netherlands Sports Federation (NOC*NSF).

The authors would like to acknowledge Dr. E. van Breda and Dr. D. Sewell for their valuable comments. Furthermore, special thanks to all the subjects who participated reliably in this study. 


\section{References}

1. Costill D, King D, Thomas R, Hargreaves $M$. Effects of reduced training on muscular power in swimmers. Physician Sports Med. 13: 94-101 1985.

2. Coyle E.F. W.H.d. Martin, S.A. Bloomfield. O.H. Lowry, J.O. Holloszy. Effects of detraining on responses to submaximal exercise. / App/ Physiol 59: 853-859 1985.

3. Coyle E.F., W.H.d. Martin, D.R. Sinacore, M.J. Joyner, J.M. Hagberg, J.O. Holloszy. Time course of loss of adaptations after stopping prolonged intense endurance training. / App/ Physiol 57: 1857-1864 1984.

4. Farrel P.E. J.H. Wilmore, E.P. Coyle. plasma lactate accumulation and distance running performance. Med Sci Sports exerc 11: 338-344 1979.

5. Gibala M.J. J.D. MacDougall, D.C. Sale. The effects of tapering on strength performance in trained athletes. Int / Sports Med 15: $492-4971994$.

6. Hickson R.C., C. Foster, M.L. Pollock, T.M. Galassi, S. Rich. Reduced training intensities and loss of aerobic power, endurance, and cardiac growth. J Appl Physiol S8: 492-499 1985.

7. Hickson R.C., C. Kanakis, Jr., J.R. Davis, A.M. Moore, S. Rich. Reduced training duration effects on aerobic power, endurance, and cardiac growth. J Appl Physiol 53: 225-229 1982.

8. Hooper S.L., L.T. Mackinnon, E.M. Ginn. Effects of three tapering techniques on the performance, forces and psychometric measures of competitive swimmers. Eur J Appl Physiol 78: 258-263 1998.

9. Houmard J. Impact of reduced training on performance in endurance athletes. Sports Med 12: 380-393 1991.

10. Houmard J.A., D.L. Costill, J.B. Mitchell, S.H. Park, T.C. Chenier. The role of anaerobic ability in middle distance running performance. Eur / Appl Physiol 62: 40-431991.

11. Houmard J.A., D.L. Costill, J.B. Mitchell, S.H. Park, R.C. Hickner, J.N. Roemmich. Reduced training maintains performance in distance runners. Int / Sports Med 11: 46-52 1990.

12. Houmard J.A., T. Hortobagyi, R.A. Johns, N.J. Bruno, C.C. Nute, M.H. Shinebarger, J.W. Welborn. Effect of short-term training cessation on performance measures in distance runners. Int / Sports Med 13: 572-576 1992.

13. Houmard J.A., J.P. Kirwan, M.G. Flynn, J.B. Mitchell. Effects of reduced training on submaximal and maximal running responses. Int / Sports Med 10: 30-33 1989.

14. Houmard J.A., B.K. Scott, C.L. Justice, T.C. Chenier. The effects of taper on performance in distance runners. Med Sci Sports Exerc 26: 624-631 1994.

15. Johns R.A., J.A. Houmard, R.W. Kobe, T. Hortobagyi, N.J. Bruno, J.M. Wells, M.H. Shinebarger. Effects of taper on swim power, stroke distance, and performance. Med Sci Sports Exerc 24: 1141-1146 1992.

16. Kuipers H., F.T.J. Verstappen, H.A. Keizer, P. Geurten, G. Kranenburg van. Variability of Aerobic Performance in the Laboratory and Its Physiologic Correlates. Sport Med 6: 197-201 1985.

17. Martin D., J. Scifres, S. Zimmerman, J. Wilkinson. Effects of interval training and taper on cycling performance and isokenetic leg strength. Int. J. Sports Med. 15: 485-461 1994. 
18. Neary J.P., T.P. Martin, D.C. Reid, R. Burnham, H.A. Quinney. The effects of a reduced exercise duration taper programme on performance and muscle enzymes of endurance cyclists. Eur J Appl Physiol 65: 30-36 1992.

19. Neufer P.D. The effect of detraining and reduced training on the physiological adaptations to aerobic exercise training. Sports Med 8: 302-320 1989.

20. Neufer P.D., D.L. Costill, R.A. Fielding, M.G. Flynn, J.P. Kirwan. Effect of reduced training on muscular strength and endurance in competitive swimmers. Med Sci Sports Exerc 19: 486-490 1987.

21. Peronnet F., D. Massicotte. Table of nonprotein respiratory quotient: an update. Can J Sport Sci 16: 23-29 1991.

22. Shepley B., J.D. MacDougall, N. Cipriano, J.R. Sutton, M.A. Tarnopolsky, G. Coates. Physiological effects of tapering in highly trained athletes. J Appl Physiol 72: 7067111992.

23. Van Handel P.J., A. Katz, J.P. Troup, J.T. Daniel, P.W. Bradley. Aerobic economy and competitive swim performance of U.S. elite swimmers. In: Swimming sciences $V$. Champaign: Human Kinetics, 1988, p. 219-227. 

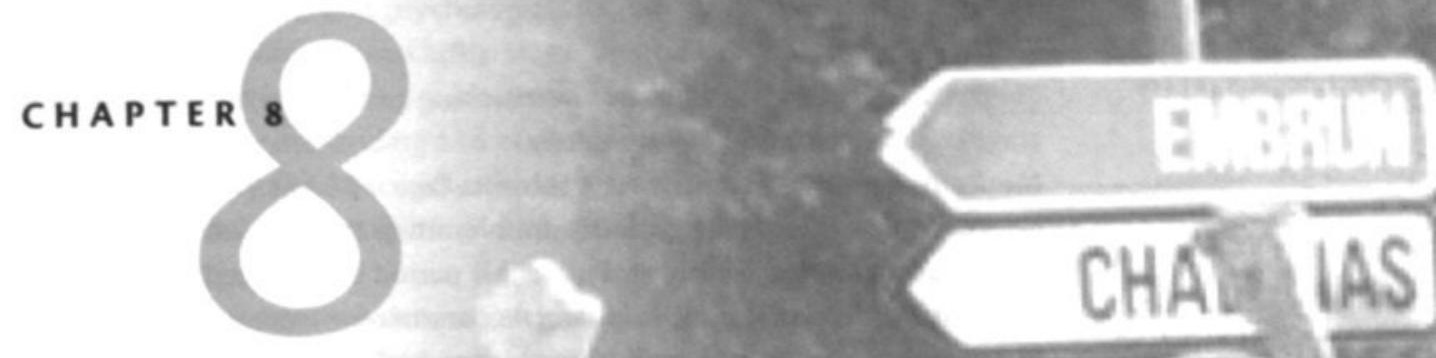

Physiological, biochemical and psychological markers for overreaching; Early markers for overreaching

\section{This Chapter has been submitted as:}
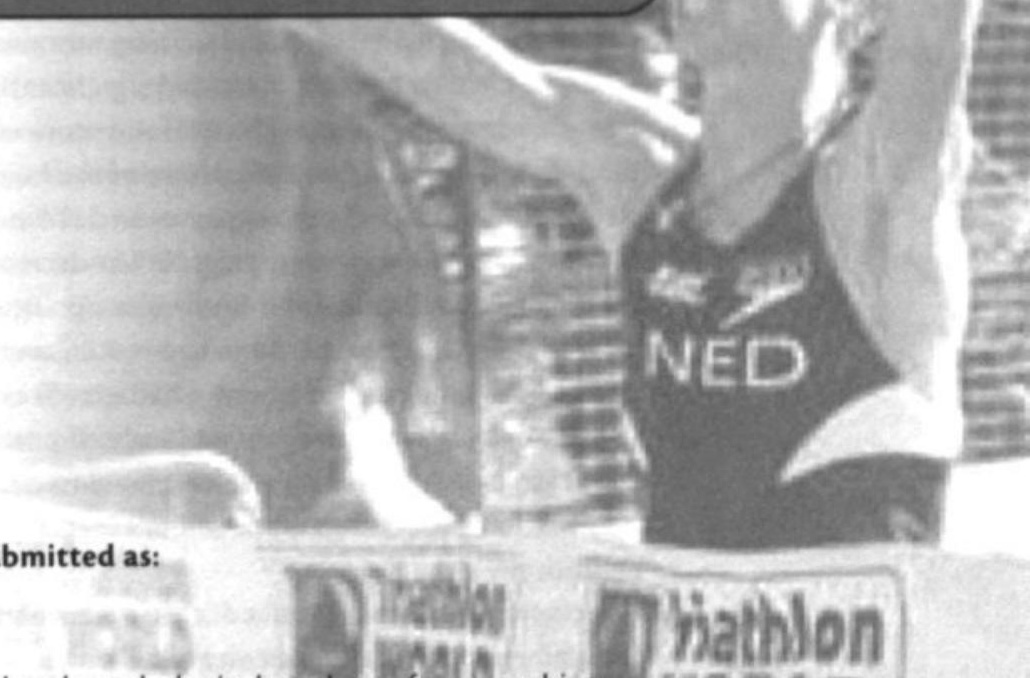

Physiological, biochemical and psychological markers of overreaching

Early markers for overreaching

G.J.W.M. Rietjens', H. Kuipers', J.J. Adam', W.H.M. Saris 1 E. van Breda (

D. van Hamont' and H.A. Keizer'
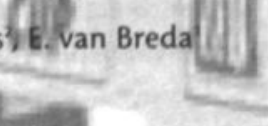

Nutrition and Toxicology Research Institute Maastricht (NUTRIM)

Department of Movement Sciences, Maastricht University, Maastricht. The Netherlands

Department of Human Biology, Maastricht University, Maastricht, The Netherlands

'Brain and Behavior Institute, Maastricht University, Maastricht, The Netherlands.

International Journal of Sports Medicine 


\section{Abstract}

Purpose: To investigate whether overreaching can be diagnosed in an early stage by a combination of parameters.

Methods: Seven well-trained male subjects (age $=25.3 \pm 4.7 \mathrm{yr} ;$ wt $=76 \pm 6.6$ $\mathrm{kg} \mathrm{VO}_{2} \max 61.1 \pm 7 \mathrm{ml} \cdot \mathrm{kg}^{\prime} \cdot \mathrm{min}^{\prime}$ ) doubled their training volume over a period of two weeks. Before and after this period subject underwent a series of tests including anthropometric parameters, incremental cycle ergometer test $\left(\mathrm{VO}_{2} \max\right.$ and $\left.\mathrm{Wmax}\right)$, time trial, blood testing (lactate, red and white blood cell profile), hormones (growth hormone (GH), insulin-like growth factor-1 (IGF-1), adreno-corticotropic hormone (ACTH), cortisol and testosterone\}, stress testing \{short insulin tolerance test (ITT), combined anterior pituitary (CAPT) or exercise\}, profile of mood state (POMS) and rate of perceived exertion (RPE) questionnaires and a cognitive reaction time test.

Results: The doubling of training volume resulted in a significant increase of the training load $(P<0.01)$, training monotony $(P<0.01)$ and training strain $(P<0.01)$. The RPE increased significantly $(P<0.01)$ during the intensified training period. Total mood score obtained from the POMS

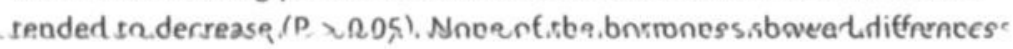
between the pre- and post exercise- and ITT measurements. During the CAPT only cortisol showed a significant decrease after the intensified training period. Hemoglobin showed a significant decrease after the intensified training where hematocrit, RBC and MCV only tended to decrease. The intensified training had no effect on physical performance (Wmax or time trial) and white blood cell profile. By contrast, reaction time results indicated that overreaching may adversely affect speed of information processing by the brain, especially for the most complex conditions.

Conclusion: From the results of the present study it can be concluded that from all endocrine variables measured only cortisol is useful as an early diagnostic marker for overtraining by using the CAPT. However, POMS, RPE and reaction time performance seems to be more indicative and sensitive markers to detect overreaching in an early stage.

Key Words: Overtraining, performance, POMS, training monitoring, hormones, reaction time

\section{Introduction}

In order to improve performance, physical training requires an optimal balance between training load (intensity and volume) and recuperation. 
Furthermore, social and psychological factors play a role in performance as well. In superbly trained athletes, however, this is not always easy to achieve. First of all training regimens that maintain or augment performance are often highly demanding and easily lead to an imbalance in the load versus recovery time. Secondly, the increasing amount of international competitions put a heavy burden on social and psychological factors as well.

If a state of general imbalance does continue for a prolonged period of time, it may eventually lead to overreaching and/or an overtraining syndrome (25). The overtraining syndrome is complex and characterized by a wide variety of symptoms (15). Maladaptations have been observed at the level of the central-nervous system, the (neuro)-endocrine system as well as at the peripheral level $(4,8,26,27)$. For example, insomnia with or without nocturnal sweating, mild to severe depression and other negative feelings in the mental state are clear indications of involvement of the central nervous and peripheral system $(20,32,36)$.

Evidence for derangements within the (neuro)-endocrine system was reported by Lehman et al. (1993) showing a diminished cortisol response to adreno-corticotropic hormone (ACTH) upon stimulation with the homologic hypothalamic-pituitary stressor corticotropin releasing hormone (CRH) (27). In contrast, however, are the results of Barron et al. (1985) who reported decreased ACTH levels in overtrained athletes stimulated with a heterologic stressor (insulin-induced hypoglycemia) (4). However, Barron et al. reported data from only four overtrained athletes and unfortunately no other studies have confirmed these results (4). Derangements of peripheral origin are often manifested as an increased susceptibility to infections caused by a malfunction of the immune system $(12,15,28)$. Also derangements of the hematological system have been reported $(24,27)$.

It is now generally accepted that a state of overreaching precedes the full blown overtraining syndrome and although several attempts have been made to diagnose the overreaching/overtraining syndrome, general diagnostic parameters have not yet been found. As mentioned above, overreaching is the initial phase of the overtraining syndrome and is characterized by increased fatigability and delayed recovery. Although there is circumstantial evidence that the physiological response to a dysbalance in training load and adequate recovery varies between individuals (41), most studies performed, have not taken into account the complex interplay between all the parameters characterizing the different systems involved.

Therefore, the present study investigates central and peripheral physiological, endocrine and psychological responses to a state of overreaching in seven well trained cyclists who experienced a two-week intensified 
training program with $100 \%$ increase in training volume.

The main aim of the present study was to establish whether early overreaching could be diagnosed by a combination of a selected number of parameters, representative for the status of the central nervous, neuroendocrine and peripheral systems.

\section{Materials and methods}

\section{Subjects}

Seven healthy, well-trained male cyclists, aged $25.3 \pm 4.7$ (mean \pm SD), volunteered to participate in this study after giving written informed consent, and the project was previously approved by the Medical Ethical Committee of the Maastricht University. All subjects completed a medical checklist. Their height was $184.6 \pm 2.7 \mathrm{~cm}$; body mass was $76 \pm 6.6 \mathrm{~kg}$ and $\mathrm{VO}_{2} \max 61.1 \pm 7 \mathrm{ml} \cdot \mathrm{kg}^{\prime} \cdot \mathrm{min}^{\prime}$. None of them was taking any medication known to affect the hypothalamic-pituitary axis (HPA).

Additionally, a group of seven healthy, well-trained, aged matched cyclists were included and served as a control group for the cognitive speed test measurements.

\section{Training}

To instigate a state of overreaching the subjects had to follow a daily program of intensified training. Before starting this two-week intervention period, the normal training program was assessed over a period of two weeks (pre-intervention period). From day one of the pre-intervention period, until the end of the two week intervention period all athletes kept a training log in which they had to fill in their training time ( $\mathrm{min})$, and the rate of perceived exertion (RPE) of the training on a 10 point Borg scale (6) $(1=$ very very low and $10=$ very very heavy). Training load, training strain and training monotony were subsequently calculated according to Foster et al. (13). Based on the average daily training time over the preintervention period, a training schedule for the intervention period was prepared. Since previous studies showed that a large increase in training volume results in overreaching or overtraining $(23,26)$, the training volume was doubled (100\%) during the intervention period. Subjects' training intensity (based on their heart rate during the pre-intervention period and the maximal cycle ergometer test) was increased by $15 \%$. In a study in horses, Bruin et al. (7) found that elimination of the recovery days during an intensified training period, induced signs of the overtraining syndrome, therefore the total amount of training hours was equally spread over all seven days of the week. In order to supervise training sessions during the intervention period, at least three training sessions per week 
were done in the laboratory. In the intervention period, training intensity of all training sessions was controlled, by heart rate registration (Polar, Kempele, Finland). To ensure an appropriate recovery after the two-week intervention period, the subjects were advised to lower the training volume during one week to $50 \%$ of their normal training load. Before and after the intensified training period, subjects underwent several tests and anthropometric measurements as described hereafter.

\section{Measurements}

Immediately before and directly after the two-week intervention period, the subjects underwent a series of tests consisting of anthropometric measurements, a cognitive speed test, operationalized by means of the finger-precuing reaction time task (30), an incremental exercise ergometer test, a performance test (time trial), a short insulin tolerance test (ITT) and a combined anterior pituitary (CAPT) test. Furthermore, an adjusted profile of mood state (POMS) (10) questionnaire was administered to each subject on a daily basis. The sequence of testing and training is illustrated in figure 1. For each subject, all testing was done at the same time of the day. Subjects had to refrain from training for at least 24 hours before each time point for data collection. At last, the subjects were asked to consume the same diet for the last 48 hours before they reported to the laboratory.

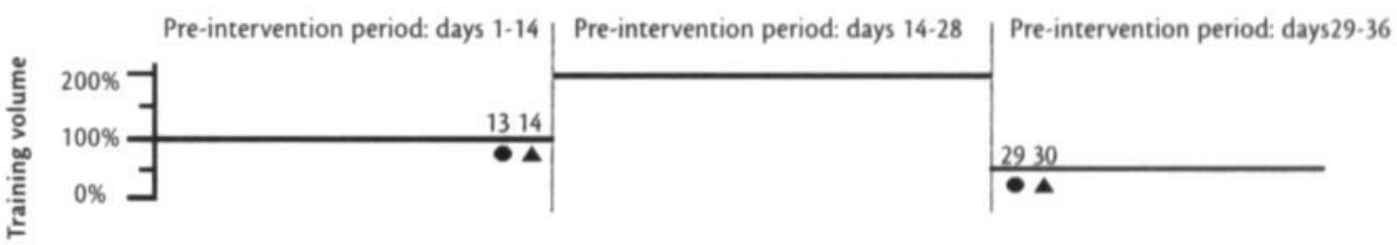

\section{FIGURE 1}

Schematic of the study design. The study started with a two weeks monitor period (pre-intervention period), followed by a two week intervention period wherein training load was $100 \%$ increased. Measurements of anthropometrie, performance and hematological variables were measured before and after the intervention period. Mood state was measured daily throughout the pre- and intervention period. Circle; anthropometric-, hematological- and incremantal exercise test. Triangle; Time trail test. 


\section{Anthropometric parameters}

Body height, body mass and percentage body fat were measured prior to and at the end of the intervention period (table 1).

Percentage body fat was calculated using the skin fold method, according to Durnin and Womersley (11).

\section{TABLE 1}

Antropometric, performance and hematological variables before and after the two weeks intensified training.

\begin{tabular}{|c|c|c|c|c|c|c|}
\hline \multirow[t]{2}{*}{ Parameter } & \multicolumn{2}{|c|}{ pre-intervention } & \multicolumn{2}{|c|}{ post-intervention } & \multirow[t]{2}{*}{ \% difference } & \multirow[t]{2}{*}{ P value } \\
\hline & mean & SD & mean & SD & & \\
\hline training volume ( $\mathrm{min} /$ week) & $420 \pm$ & 204 & 870 & \pm 295 & 107.14 & $<0.01$ \\
\hline \multicolumn{7}{|l|}{ Anthropometrie } \\
\hline Body Mass (kg) & $76 \pm$ & 6.67 & $5.8=$ & \pm 6 & -0.26 & 0.66 \\
\hline Body Fat (\%) & $13.3 \pm$ & 2.5 & 12.9 & \pm 2.5 & -3.01 & 0.32 \\
\hline \multicolumn{7}{|l|}{ Performance test } \\
\hline Time crial (sec) $(n=7)$ & $1817 \pm$ & 107 & $1847=$ & \pm 161 & 1.65 & 0.86 \\
\hline Time trial (sec) $(n=5)$ & $1747 \pm$ & 84 & 1810 & \pm 106 & 3.61 & $<0.01$ \\
\hline Maximal workload (Watt) & $325 \pm$ & 5.7 & $337 \pm$ & \pm 5.5 & 3.69 & 0.08 \\
\hline Oxygen uptake $\left(\mathrm{ml}^{\prime} \cdot \mathrm{kg}^{\prime} \cdot \mathrm{min}^{\prime}\right)$ & $58.3 \pm$ & 7.8 & $58.7 \pm$ & \pm 6.6 & 0.69 & 0.86 \\
\hline Peak blood lactate ( $\mathrm{mmol} / \mathrm{I})$ & $11.5 \pm$ & 3.1 & $11.2 \pm$ & \pm 2.4 & -2.6 & 10.86 \\
\hline \multicolumn{7}{|l|}{ Blood Profile } \\
\hline $\mathrm{Hb}(\mathrm{mmol} / \mathrm{I})$ & $8.86 \pm$ & 0.36 & $8.46 \pm$ & \pm 0.31 & -4.51 & $<0.01$ \\
\hline Hct $(\%)$ & $0.43 \pm$ & 0.03 & $0.41 \pm$ & \pm 0.03 & -4.65 & 0.25 \\
\hline$R B C(p l)$ & $4.67 \pm$ & 0.24 & $4.54 \pm$ & \pm 0.28 & -2.78 & 0.2 \\
\hline $\operatorname{MCV}(f)$ & $92.76 \pm$ & 6.31 & $89.5 \pm$ & \pm 5.03 & -3.51 & 0.33 \\
\hline WBC & $5.3 \pm$ & 1.33 & $5.61 \pm$ & \pm 1.68 & 5.85 & 0.79 \\
\hline Leucocytes & $1.98 \pm$ & 0.59 & $1.94 \pm$ & \pm 0.6 & -2.02 & 0.14 \\
\hline Monocytest & $0.98 \pm$ & 0.82 & $0.33 \pm$ & \pm 0.08 & -66.33 & 0.1 \\
\hline Granulocytes & $2.32 \pm$ & 0.81 & $2.73 \pm$ & \pm 0.67 & 17.67 & 0.48 \\
\hline
\end{tabular}

Values are mean $\pm S D$, significance was accepted at $\mathrm{P}<0.05$ level. 


\section{Cognitive speed test}

Experimental set up

\section{Apparatus and stimuli}

Stimuli were presented on a standard video display monitor controlled by an IBM-AT computer. Responses were made by pressing one of four keys of the keyboard (the two left-most and two right-most keys on the bottom row of the keyboard operated by the index and middle fingers of both hands). Viewing distance was held constant at about $50 \mathrm{~cm}$. The computer was housed in a normally lit room, and was used to control the presentations of the stimulus displays and to record response latencies and accuracies (2).

Stimuli were plus (+) signs presented in the standard character set of the computer. The stimulus display consisted of a warning signal, a cue signal, and a target signal, with the entire display centered on the viewing monitor. The warning signal was a row of four plus signs (figure 2).

After a delay of $750 \mathrm{~ms}$, the cue signal appeared immediately below the warning signal. The cue consisted of plus signs either in all four positions indicated by the warning signal (uncued condition) or in only two of the four possible positions (hand-cued, finger-cued, neither-cued conditions).

\section{Finger Precuing Reaction time Task}

\begin{tabular}{|c|c|c|c|c|}
\hline & Uncued & Hand-Cued & Finger-Cued & Neither-Cued \\
\hline \multirow[t]{2}{*}{$\begin{array}{l}\text { Warning } \\
\text { Cue } \\
\text { Target } \\
\text { Responses }\end{array}$} & $\begin{array}{l}++++ \\
++++ \\
+\end{array}$ & $\begin{array}{l}++++ \\
++ \\
+ \\
+\end{array}$ & $\begin{array}{c}+++ \\
++ \\
+ \\
\square=\square\end{array}$ & $\begin{array}{l}++++ \\
+\quad+ \\
+ \\
\text { घ }\end{array}$ \\
\hline & & $\begin{array}{r}+++ \\
+ \\
+ \\
\square \square \square\end{array}$ & $\begin{array}{rr}+ & + \\
+ & + \\
& + \\
\text { Q } & +\end{array}$ & $\begin{array}{c}+++ \\
+\quad+ \\
+ \\
\square \square\end{array}$ \\
\hline
\end{tabular}

\section{FIGURE 2}

Schematic representation of the finger precuing reaction time task and the four preparation conditions. The black squares indicate the possible responses indicated by the cue, and thus reflect the number and type of prepared responses. Note that in allconditions only one response was actually required, namely the finger response indicated by the single target stimulus. 
We distinguished four precue or preparation conditions. In the hand-cued condition, the precue specified two fingers on the same hand (e.g., the left-middle and left-index fingers). In the finger-cued condition, the precue specified the same finger on different hands (e.g., the left-index and rightindex fingers). In the neither-cued condition, the precue specified different fingers on different hands (e.g., the left-middle and right-index fingers). Also, an uncued condition was included. Here, the "precue" provides no advance information (it contains plus signs in all four possible stimulus locations), and thus precludes selective preparation of any combination of two finger responses. This condition is a control condition.

After a variable (preparation) interval (i.e., 100 or $2000 \mathrm{~ms}$ ) the target signal (one single plus sign) appeared immediately below the cue row, in a position always indicated by the cue. The subject's task was to respond as quickly as possible to the position in which the target signal occurred by pressing the appropriate response key. Target signal and response key were mapped onto each other in a spatially compatible manner, such that a target appearing in the left-most position was to be responded to with the left middle finger pressing the left-most response key, etc. An intertrial interval of $1 \mathrm{~s}$ separated the response in a trial from the start of the next trial.

\section{Procedure}

Two preparation intervals were used (100 and $2000 \mathrm{~ms}$ ). Each subject received a block of 80 trials for each preparation interval. A rest period of $3 \mathrm{~min}$ was provided between blocks. Within a block of 80 trials, there were 20 trials for each of the four cue conditions. The order of these cuing conditions within a block of 80 trials was random. Twenty-five practice trials preceded each block of 80 test trials.

Participants were informed regarding the nature of the task and were explicitly told to take advantage of the cue stimulus. They were instructed to react as quickly as possible to the target stimulus by pressing the correct response key. Error feedback was provided on individual trials.

\section{Profile of Mood States (POMS).}

A shortened version of the original POMS questionnaire (10) was daily administrated during the entire study in order to quantify the influence of intensified training on mood state. The adjusted POMS assessed only tension, depression, anger, vigor and fatigue. The total mood score was measured by summing the scores of the negative moods (tension, depression, anger and fatigue) and subtracting the vigor score. From this daily score a weekly average score was calculated. The higher this mood score, the worse the mood state. Furthermore the average weekly score of each individual item was analysed. 


\section{Performance tests}

\section{Incremental exercise test.}

After insertion of a Teflon catheter in an antecubital fore arm vein, the subject rested in supine position for $\mathbf{2 0}$ minutes. Blood samples for lactate, adrenocorticotropic hormone (ACTH), cortisol, thyroid-stimulating hormone (TSH), Insulin-like growth factor-1 (IGF1) and growth hormone $(\mathrm{GH})$ were drawn at rest and immediately after the termination of the test. From the sample obtained before the test a red \{hemoglobine $(\mathrm{Hb})$, hematocrit ( $\mathrm{Hct})$, red blood cell count (RBC) and Mean Corpuscular Volume (MCV)\} and white blood cell \{white blood cell count (WBC), leucocytes, monocytes, granulocytes\} profile was analysed as well. The subjects had to complete the incremental exercise test on an electromagnetically braked cycle ergometer (Lode Excalibur Sport, Lode, The Netherlands) that was calibrated at rpm values between 70 and $100 \mathrm{rpm}$. The initial workload of the maximal workload test was set at $1 \mathrm{~W} / \mathrm{kg}$ body weight (BM), and increased with $0.5 \mathrm{~W} / \mathrm{kg} \mathrm{BM}$ every three minutes until a volitional exhaustion. The maximal workload (Wmax) was defined as the last completed work load $(W)$ plus the fraction of time spent in the final work rate multiplied by workload of the last completed step (22). Throughout the test, gas exchange (Oxycon B, E.Jaeger $\mathrm{GmbH}$, Höchberg. Germany) and heart rate (Polar, Kempele, Finland) were measured continuously.

\section{Time Trial.}

To obtain an additional reproducible measure of performance, a simulated time trial was used (21). The measure of performance was the time to complete an amount of work determined by the following formula:

\section{Target amount of work $(J)=75 \% \mathrm{Wmax} \cdot 1800 \mathrm{sec}$}

During this test the subjects were not informed about their workload, cadance, heart rate or elapsed time.

\section{Hormone provocation tests}

\section{Insulin Tolerance Test.}

In order to study the endocrine response during a hypoglycemia challenge test, a venous insulin tolerance test (ITT) was used (1). The subjects reported to the laboratory after an overnight fast. They had to refrain from physical exercise 24 hours preceding the ITT. To obtain arterialized blood samples the forearm and hand were held in a heated air box set at $60^{\circ} \mathrm{C}$. In the contra lateral arm an intravenous catheter was inserted in the antecubital vein for the injection of insulin. After $\mathbf{3 0}$ minutes of rest 0.04 units / Kg BM short acting insulin (Human Actrapid Insulin", Novo Nordisk 
A/S, Denmark) was injected. Blood samples for ACTH, cortisol, TSH and $\mathrm{GH}$ were drawn just before, and 10, 20, 40, 60, 80 and 100 min after the insulin injection. Blood glucose was measured every 2.5 minutes during the first $\mathbf{4 0} \mathrm{min}$ and every $\mathbf{1 0} \mathrm{min}$ thereafter. If blood glucose levels dropped below $2.0 \mathrm{mmol} / \mathrm{l}$ or if other symptoms of severe hypoglycemia occurred, the test was terminated and a bolus injection of $20 \mathrm{ml} \mathrm{20 \%}$ glucose solution in saline was administered intra-venously.

\section{Combined Anterior Pituitary Test.}

On a separate day, the athletes reported to the laboratory at 8.30 A.M. after an overnight fast. Because the ITT test does not discriminate between hypothalamic and pituitary dysfunction, an additional test was necessary. The Combined Anterior Pituitary Test (CAPT) is used to assess pituitary function. In the present study this test was used to distinguish between disturbances at pituitary or hypothalamic level. After insertion of a catheter in both antecubital veins of both arms, the athlete was seated for $\mathbf{2}$ hours. Ten minutes after the insertion of the catheter, a bolus injection with a physiological dose of releasing hormones $(33.3 \mu \mathrm{g} \mathrm{CRH}$, $17.0 \mu \mathrm{g}$ GhRH (somatorelineactaat) and $0.066 \mathrm{mg} \mathrm{TRH}$ in $10 \mathrm{ml}$ saline was given. Blood $(10 \mathrm{ml})$ for analvsis of ACTH. corrisol. JSH and GH. was sampled just before the bolus injection, and 10, 20, 40, 60, 80 and $100 \mathrm{~min}$ after the injection.

\section{Blood sample processing and analysis.}

Blood samples $(1 \mathrm{ml})$ for lactate were collected in heparinized tubes, centrifuged at $5,000 \mathrm{rpm}$ at $4^{\circ} \mathrm{C}$. and the plasma frozen at $-20^{\circ} \mathrm{C}$ until analysed on a semi-automatic analyser (Cobas FARA, La Roche, Basel, Switzerland). Red and white blood cell samples ( $4 \mathrm{ml}$ ) were collected in EDTA tubes and analysed immediately (Coulter MD18, Miami, USA). Four $\mathrm{ml}$ of blood was collected for Cortisol, TSH, GH and IGF-1 analysis in serum tubes, and centrifuged at $5,000 \mathrm{rpm}$ at $4^{\circ} \mathrm{C}$ after clotting. The resulting plasma was collected and stored at $-20^{\circ} \mathrm{C}$. Samples for ACTH were collected in pre-cooled EDTA tubes and immediately centrifuged at $5000 \mathrm{rpm}$ at $4^{\circ} \mathrm{C}$. Plasma for ACTH was stored at $-20^{\circ} \mathrm{C}$. All samples from one subject were analysed in one assay to avoid interassay variability.

Sequential Immunometric Assays were used to determine blood concentrations of cortisol (Chiron Diagnostics Corporation, East Walpole, MA, USA), GH (Nichols Institute Diagnostics San Juan Capistrano, CA, USA) and ACTH (Euro/DPC, United Kingdom). Variances for the chemiluminescence assays were: cortisol; intra-assay $4.5 \%$, inter-assay $6.4 \%$, $\mathrm{GH}$; intra-assay $4.0 \%$, inter-assay $9.2 \%$, and $\mathrm{ACTH}$; intra-assay $5.9 \%$, interassay 3.9\%. Quantification of IGF-1 was performed using an Immunoradiometric Assay (IRMA) (DSL-5600 ACTIVE", DSL Deutschland GmbH, 
Germany) with an intra-assay variance of $3.0 \%$ and an inter-assay of $1.5 \%$. A radioimmunoassay (RIA) was used for the determination of plasma TSH. The intra- and inter assay coefficients of variation were $2,8 \%$ and $4.9 \%$, respectively.

\section{Statistics}

Cognitive speed test: RTs less than $150 \mathrm{~ms}$ or in excess of $1.5 \mathrm{~s}$ were considered outliers and were excluded from data analyses; less than $0.3 \%$ of the RTs were removed using this criterion. Mean correct RTs and proportions of errors were calculated for each subject as a function of preparation condition, preparation interval, and time (pre vs. post test). A split-plot analyses of variance (ANOVA) (Keppel, 1982) was performed on mean RTs and percentage errors with Intervention (control vs. intervention) as between-subject variable, and Preparation Condition (uncued, hand-cued, finger-cued, neither-cued), Preparation Interval (100 and $2000 \mathrm{~ms}$ ), and Time (pre and post intervention) as within-subject variables. Whenever appropriate, the tests involving the within-subject factor(s) were adjusted for heterogeneity of variance and covariances using the Huynh-Feldt corrected significance values. Post-hoc analyses were carried out using Tukey's honestly significant procedure.

Other parameters: All data are presented as means \pm standard deviation (SD). To evaluate differences between the total pre and post intervention hormone secretion, areas under the curve were measured during the ITT and CAPT, respectively. A Wilcoxon signed rank test for related pairs was applied to identify differences at each time point. All other test data were treated for significance with the Wilcoxon signed rank test for related pairs. Statistical significance was set at $P<0.05$.

\section{Results}

All subjects successfully completed the two weeks of the intensified training period. The average amount of training during the pre-intervention period was $420 \pm 204 \mathrm{~min}$ per week (60 min/day). During the next two weeks (intervention period) the subjects doubled the training volume to $872 \pm 295$ minutes per week ( $825 \pm 279 \mathrm{~min}$ and $939 \pm 378 \mathrm{~min}$ for wk 1 and wk 2 , respectively). On a daily basis this means that the training load increased from an average of $60 \mathrm{~min}$ during the pre-intervention period to an average of $117 \mathrm{~min}$ in week 1 and $134 \mathrm{~min}$ in week 2 . This increase in training volume resulted in a significant increase in the individual training load ( $\pm 100 \%$ increase, $P<0.01$ ), training strain ( $\pm 220 \%$ increase, 
$P<0.02)$, and training monotony $( \pm 85 \%$ increase, $P<0.01)$. Body mass $(76.0 \pm 6.5$ vs. $75.8 \pm 6.0 \mathrm{~kg})$ and body fat $(13.3 \pm 2.5$ vs. $12.9 \pm 2.5 \%)$ was not significantly changed after the two weeks of intense training.

\section{Performance tests}

Intensifying the training load did not induce any significant changes in Wmax ( $4 \%$ increase, $P=0.08), \mathrm{VO}_{2} \max (1 \%$ increase, $\mathrm{P}=0.86), \mathrm{HFmax}$ ( $4 \%$ decrease, $P=0.13$ ) and maximal lactate ( $3 \%$ decrease, $P=0.93$ ) attained during the incremental exercise test. The time to complete the pre-set amount of work was not significantly different ( $1 \%$ increase, $\mathrm{P}=0.84$ ) before and after the intervention period. It has to be kept in mind however, that five out of the seven subjects increased their time to complete the pre-set amount of work. It is interesting to know that the two other subjects had never performed a time trial before.

\section{Psychological variables}

Profile of Mood State (POMS).

Although not significant, the two weeks of increased training volume demonstrated a trend towards an increased average mood score, reflecting an increase in a worse mood state $(35.8,39.7$ and 38.7 for base line, week 1 and week 2 of the intensified training respectively). After analyzing the five sub scales of the POMS separately, none of them appeared to be significantly different compared to the base line score. However, despite an absence of significant differences five of the subjects showed sustained increased mood and fatigue score during the intensified training period. Rating of perceived exertion (RPE).

The mean RPE score increased significantly from $4.0 \pm 1.4$ in the pretraining period to $6.3 \pm 1.5$ in the first week of the intensified training. The mean RPE score decreased slightly during the second week of the intensified training period $(5.8 \pm 1.7)$ but remained significantly higher compared to the pre-intervention period mean value.

\section{Cognitive speed test}

RTs were shorter in the post-test than in the pre-test ( $390 \mathrm{vs.} 404 \mathrm{~ms}$, respectively) $(F(1,10)=3.99, P<.05)$. Most likely, this effect is due to a learning or practice effect caused by performing the finger-precuing task two times (before and after the intervention). Importantly, this learning effect was qualified by a significant three-way interaction involving the factors Intervention, Preparation Condition, and Time $(F(3,30)=3.14$, $P<.05)$. This interaction indicated that the learning effect on RT was larger for the control group than for the intervention group $(P<.05)$ but only for the most difficult conditions (i.e, the uncued and neither-cued conditions), with the hand- and finger-cued conditions showing similar 
learning effects in both groups (see Figure 3). In other words, the negative effect of overtraining showed up as a failure to generate a learning effect in the uncued and neither-cued conditions.

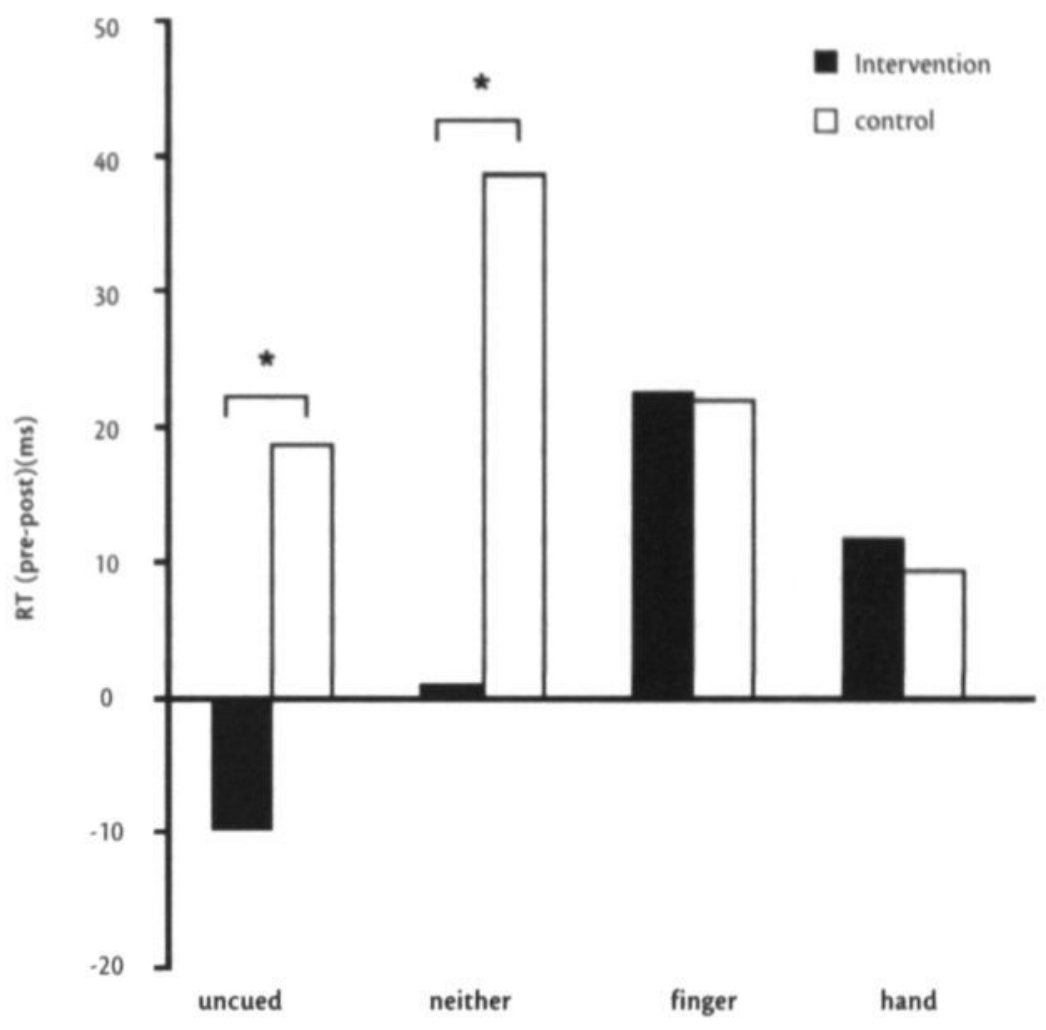

\section{FIGURE 3}

The reaction time (RT) difference in the post-test relative tot the pre-test fot the control and intervention group as a function of preparation condition. Positive numbers denote shorter RT's in the post-test than in the pre-test (i.e., a learning effect); negative numbers denote longer RT's in the post-test than in the pre-test (i.e., an interference effect). " significant difference between intervention group and control group $(P<0.05)$.

Mean error rate was $4.6 \%$. There were no significant differences between the control group and the intervention group $F(1,10)=2.31, P>.1$ nor were there significant differences before and after the intervention $(F(1,10)=2.62, P>.01)$.

Subjects made most errors in the uncued and neither cued conditions (Mean $=4.6 \%$ and $7.6 \%$, respectively), fewest in the hand-cued condition 
(Mean $=1.0 \%$ ), and an intermediate number of errors in the finger-cued condition (Mean $=3.2 \%)(F(3,30)=4.64, P<.01)$. Thus, generally, the conditions that showed the longest RTs also produced the most errors.

\section{Endocrine variables}

Maximal workload test.

Resting and maximal values of cortisol and TSH tended to be lower whereas $\mathrm{GH}$ and $\mathrm{ACTH}$ showed a small increase after the intensified training period. However, these differences during rest and at maximal exercise were not significantly different. Furthermore the difference between rest en maximal values remained unaffected either after two weeks.

\section{The insulin tolerance test.}

In all subjects, the injection of insulin induced signs of hypoglycaemia (sweating, drowsiness and hunger) and average nadir blood glucose level were approximately $2.1 \pm 0.2 \mathrm{mmol} / \mathrm{l}, 20$ to $25 \mathrm{~min}$ after the insulin injection. No differences in plasma glucose concentrations at any time point could be observed between the pre and post test. The endocrine data from the insulin tolerance test are presented in figure 4. No significat

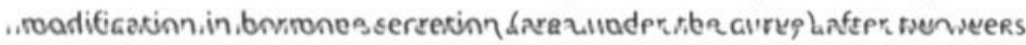
of intensified training could be found.
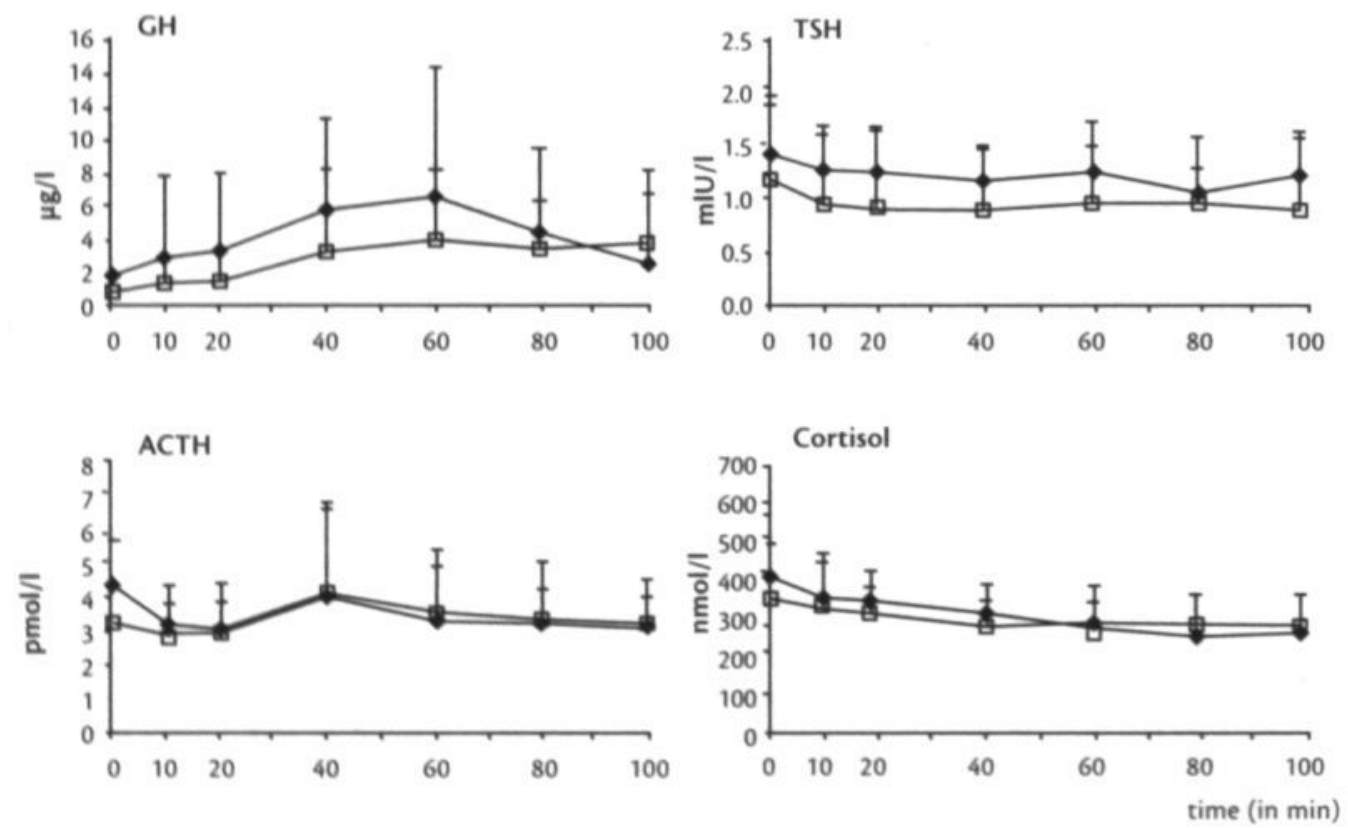

FIGURE 4

Insulin Tolerance Test: dynamics of mean ( \pm SD) of growth hormone, TSH, ACTH and cortisol before $(-)$ and after $(\square)$ two weeks of intensified training. 


\section{The Combined Anterior Pituitary Test.}

Mean plasma concentrations of $\mathrm{GH}, \mathrm{TSH}, \mathrm{ACTH}$ and Cortisol obtained during the CAPT are presented in the figure 5. Cortisol showed a significant decrease in the area under the curve after the two weeks of intensified training. All other hormones tended to decrease as well but this decrease was not significant.
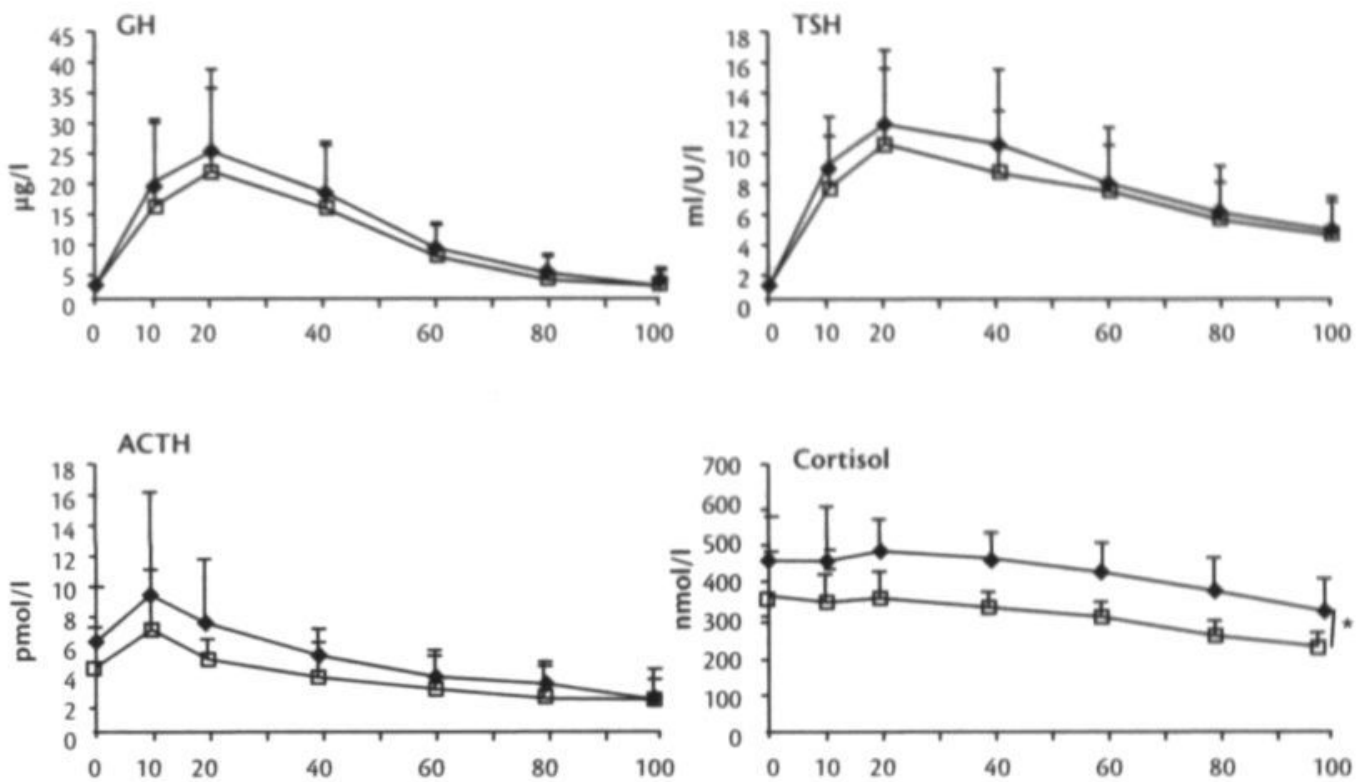

\section{FIGURE 5}

Combined Anterior Pituitary Test: dynamics of mean ( \pm SD) of growth hormone, TSH, ACTH an cortisol before $(\bullet)$ and after $(\square)$ two weeks of intensified training. The cortisol response was significantly reduced $(P<0.05)$ after the two weeks intensified training:

\section{Hematological variables}

White blood cell profile.

An overview of the white blood cell variables is given in table 1. All values for each subject were within the normal range. The total number of leukocytes and the sub-population of the leukocytes did not significantly change after the intensified training.

\section{Red blood cell profile.}

All values for red blood cell variables were within the established normal ranges for men (table 1). Only hemoglobin showed a significant $4.5 \%$ 
decrease $(P<0.01)$ after the two weeks of training. Although not significan mean Hct (6\%), RBC (3\%) and MCV (3.5\%) showed a decrease after the intervention period.

\section{Discussion}

The main purpose of the present study was to find potential early marke to identify overreaching and thus to prevent athletes reaching the state $i$ the "overtraining syndrome". The clinical features of this syndrome suggests the involvement of the higher brain centers, the hypothalamicpituitary-adrenal, hypothalamic-pituitary- $\mathrm{GH}$ axis and peripheral organs, therefore, variables indicative for these systems have been measured. To induce a state of overreaching, the normal training volume of seven well-trained cyclists was doubled. Although we were unable to show significant changes in physical performance which could be expected because of increased training, the doubled training volume resulted in a significant increase in training load, strain and monotony. This suggests a imbalance between training and recovery and indicates that the subjects

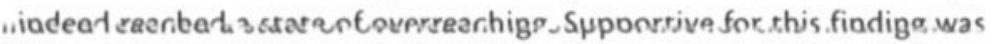
that after the two weeks intensified training the exercise time of the time trial increased in five out of seven subjects, whereas RPE was significantly increased in all subjects during this period. In contrast, other performance indices such as the Wmax obtained from the incremental bicycle ergometer test, did not decrease during the two weeks of intensified training. This is in line with data from Bruin in horses (7). Thus, it turned out that an incremental performance test is not sensitive enough to detect a small performance decrement during overreaching or over-training in its earliest stage. This is most likely caused by the normal day-to-day variability in performance obtained by incremental bicycle ergo-meter tests (i.e. approximately 5\%) (22), which is higher than the small differences in performance (some percents) due to intensified training, at least in elite athletes.

To get more insight into exercise-induced changes in brain functioning, we used two different approaches. Firstly, we asked our subjects to fill in an adjusted POMS questionnaire, which provides data on mood state, secondly the subjects underwent a reaction task, which is indicative for cognitive function. No significant changes in the POMS global mood score or in one of the five sub-scales (tension, depression, anger, vigor and fatigue) were found. Based on these results it might be argued that the POMS is not a strong early marker for overreaching. This contrasts with findings of several (over)training studies where the POMS was mentioned as an efficient model to prevent overtraining in swimmers, runners and canoeists $(5,32,33,40)$. However, also the results of the present study 
indicate that the POMS can be useful in detection overreaching.

First of all there was a great difference in the inter-individual global POMS score. Six subjects showed an increase in the global mood score after one week of increased training load, reflecting a decrease in their mood state. This number of subjects remained the same in the second week of the intensified training but in three subjects the global mood score increased further whereas in three other, a small decrease was observed.

Examination of the five sub-scales (tension, depression, anger, vigor and fatigue) did not cause any significant change in any of the items. Despite a lack of significance in the mean score, six out of the seven subjects showed an increase in their anger score and a decrease in the vigor score already after one week of intensified training. Furthermore, four athletes had elevated fatigue scores. This resulted in a trend towards flattening of the classical ice berg profile normally found in athletes (31) caused by a decrease in the vigor score and an increase in the fatigue score (figure 6). In a previous study of Morgan et al. a strong dose-response relationship between training load and mood disturbance was found and an inverted ice berg profile (together with decrement in performance) was associated with an overtraining syndrome (32). A possible explanation for this discrepancy between our study and others is the limited number of subjects in the present study and the wide scatter of the data, probably caused by the relatively short intervention period. Furthermore, it is possible that the shortened version of the POMS is less sensitive as the original one.

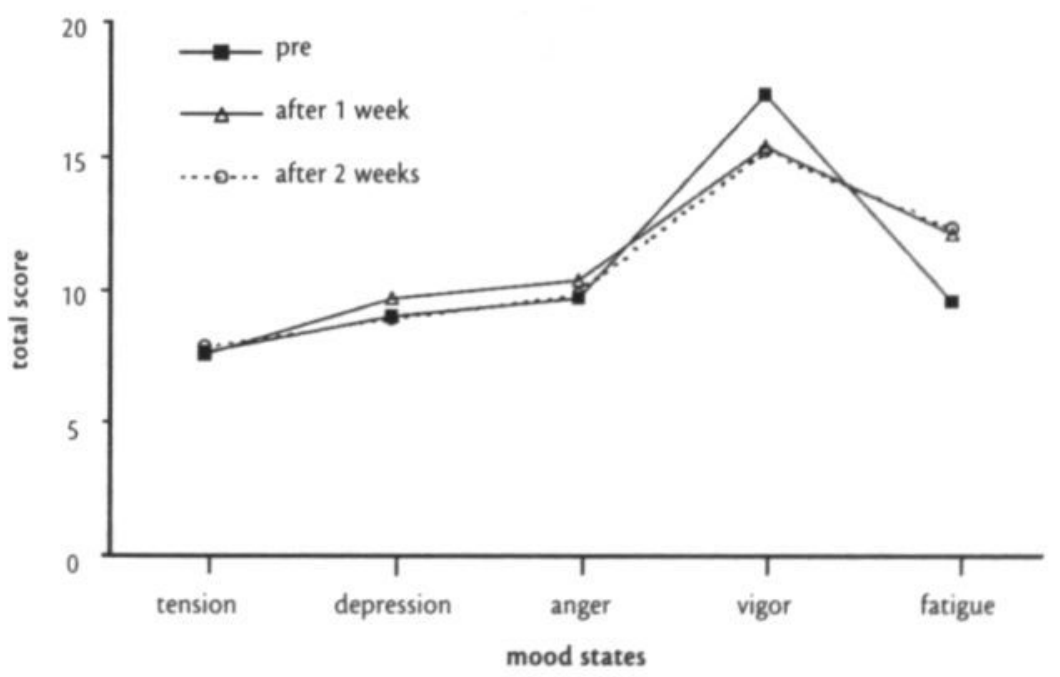

\section{FIGURE 6}

Changes in the "iceberg profile" as a result of the increased training volume during the intervention periode. 
In line with the negative changes in mood state, the increased training volume also resulted in a training being perceived as more effortful. This finding is in line with two other studies that found a progressive increase in the RPE after increasing the training volume in swimmers $(33,34)$. We used in the present investigation, to the best of our knowledge for the first time a reaction time task, which is indicative for cognitive function. The latter was operationalized by means of the finger-precuing reaction time task. This task is a complex task because it requires subjects to quickly process cue information, to select and prepare a subset of responses, and finally to select and execute a fast finger (i.e., button-press) response. Certain subsets of fingers are easier to prepare than others, with fingers on one hand (i.e., hand-cued) most easy to prepare and different fingers on different hands (i.e., neither-cued) most difficult. Consistent with this fact, the present study found that repeated performance of the finger-precuing task during the pre-test and post-test sessions benefitted more the difficult neither-cued condition than the relatively easy hand-cued condition. This was found for the control group. The overreached group, on the other hand, exhibited a different pattern. That is, whereas the overreached group did show a similar practice benefit for the hand-cued (and finger-cued) condition, as did the control group, it demonstrated no practice benefit for the more difficult neither-cued (and uncued) condition. From this result it appears that overreaching has a negative impact on the more difficult conditions in the finger-precuing reaction time task, with the more easy conditions being insensitive to overreaching. This outcome suggests that task complexity is an important mediating variable in the relationship between overreaching and brain functioning.

One of the aims of this study was to investigate whether increases in training load, which may lead to overreaching, do affect the physiological neuro- endocrine response to standard stress tests. For this purpose, we used beside the exercise-induced endocrine response, the short insulin tolerance test (ITT) and the combined anterior pituitary test (CAPT). Insulin-induced hypoglycemia is considered as a heterologous stressor (in contrast to exercise, which is a homologous stressor). In healthy subjects an insulin-induced hypoglycemia activates hypothalamic neurons to stimulate the pituitary release of $\mathrm{ACTH}, \mathrm{GH}$ and $\mathrm{TSH}$, and consequently plasma levels of these hormones will increase, as will plasma cortisol level (39). This hypoglycemic reaction is described to be rather robust (35). Barron et al. introduced the ITT for the first time in four overtrained athletes (4). In that study, four athletes with a likely overtraining syndrome were injected with $0.1 \mathrm{IU} / \mathrm{kg} \mathrm{BM}$ of insulin. A decreased responsiveness of the hypothalamic-pituitary system to hypoglycemic stress and consequently lowered plasma $\mathrm{GH}, \mathrm{ACTH}$ and cortisol responses were demonstrated in 
these athletes in the state of overtraining. Since TRH and $\mathrm{LHRH}$-induced TSH, PRL, LH and FSH responses were normal, it was concluded that hypothalamic or higher brain center rather than pituitary function caused the impaired response to an insulin-induced hypoglycemia.

In the present study no significantly changes in any of the measured hormones were found. This may be attributable to the fact that our athletes were not suffering from an overtraining syndrome. An important finding of the present study is that an insulin dose of $0.1 \mathrm{UI} / \mathrm{kg} \mathrm{BM}$ will almost inevitably lead to severe hypoglycemia. In a pilot study preceding the present study, initially we started with the same dose as Barron et al., but because all subjects ended with severe hypoglycemia, the dose was decreased to $0.04 \mathrm{UI} / \mathrm{kg} \mathrm{BM}$.

The results from the CAPT demonstrated only a significantly lowered cortisol response as reflected by the area under the curve. All other hormones showed no significant changes, albeit that there was a tendency for a lowered response. This indicates that intensified training induced a decreased responsiveness of the adrenal cortex. In an experimental overtraining study Lehmann and co workers found increased ACTH, GH, prolactin, $\mathrm{LH}$ and $\mathrm{FSH}$ plasma levels after an injection with releasing hormones (27). After two additional weeks of high-volume training in trained road cyclists at the end of their competitive season a decreased pituitary ACTH response to corticotrophin-releasing hormone $(C R H)$ was observed (18).

Hematological changes are sometimes reported in overreached or overtrained athletes $(24,27)$ were others failed to find any changes $(38)$. In the present study significant differences in blood biochemical parameters after the intervention period were limited to a reduction in $\mathrm{Hb}$. $\mathrm{HCt}, \mathrm{RBC}$ and MCV tended to be lower as well, however all values were within the normal range. Red blood cell values at the lower limit of the normal range have often been reported in endurance athletes not suffering from overreaching or overtraining and are more likely a desirable adaptation of endurance training $(9,19,37)$. Based on the results of the present study, it can be concluded that changes in the red blood cell profile are not specific to detect overtraining or overreaching and therefore not useable as an early marker for these situations. In overtrained athletes, susceptibility for infections and a frequent illness is often indicated. However speculative, there was a remarkable high number of subjects $(n=4)$ that suffered from influenza after the intensified training period. Though, no changes in the white blood cell profile were found after the intensified training period. This finding is in line with the findings of Fry et al (1992a, b), Mackinnon et al (1997) and Gabriel et al (1998) $(14,16,17,29)$. Despite findings of Lehmann et al. (1996) and Baj et al. (1994) who reported a decrease in number of leucocyte after a period of 
strenuous training $(3,28)$ it is concluded that the white blood cell distribution is not consistent and sensitive enough to detect overreaching in an early stage.

Overall the results do not show substantial changes in the measured biochemical variables as indicator of overreaching or overtraining. A possible explanation for this lack of changes could also be the design of the study, which is normally used to standardize the measurements namely to prescribe no training in the 24 hours preceding the test. Perhaps this artificial rest period could benefit recovery processes in such a way that no signs of overtraining can be found.

In conclusion, the data of the present study show that overreaching is difficult to detect in its early stage, using laboratory tests. In addition, the hypoglycemic stress test failed to alter the physiological response during overreaching and an early state of overtraining. However, an important finding was that the adrenal cortex was less responsive to a stimulus of adrenocorticotropic hormone. Although the mean scores of the POMS failed to show significant changes, still the POMS on an individual basis and RPE appear to be a more indicative and sensitive marker for detecting overreaching/overtraining in an early stage compared to the measured biochemical variables. A novel finding of the present study is that overreaching is associated with early changes in cognitive test performance.

\section{Acknowledgement}

This research was supported by a grant from the Netherlands Olympic Committee and Netherlands Sports Federation (NOC*NSF).

The authors express their gratitude to Dr D. Sewell and Dr A.D.M. Kester for their valuable comments and to all the subjects for their tremendous amount of exercise. 


\section{References}

1. Consensus guidelines for the diagnosis and treatment of adults with growth hormone deficiency: summary statement of the Growth Hormone Research Society Workshop on Adult Growth Hormone Deficiency. J Clin Endocrinol Metab 83: 379-381. 1998.

2. Adam J., F.G. Paas, J.C. Teeken, E.M. van Loon, M.P. van Boxtel, PJ. Houx, J. Jolles. Effects of age on performance in a finger-precuing task. J Exp Psychol Hum Percept Perform 24: 870-831. 1998.

3. Baj Z., I. Kantorski, E. Majewska, K. Zeman, L. Pokoca, E. Fornalczyk, H. Tchorzewski, Z. Sulowska, R. Lewicki. Immunological status of competitive cyclists before and after the training season. Int / Sports Med 15: 319-324. 1994.

4. Barron G.L., T.D. Noakes, W. Levy, C. Smith, R.P. Millar. Hypothalamic dysfunction in overtrained athletes. J Clin Endocronilogy and Metabolism 60: 803-806 1985.

5. Berglund B., H. Safstrom. Psychological monitoring and modulation of training load of world-class canoeists. Med Sci Sports Exerc 26: 1036-1040 1994.

6. Borg G.A. Psychophysical bases of perceived exertion. Med Sci Sports Exerc 14: 377-381 1982.

7. Bruin G., H. Kuipers, H.A. Keizer, G.J. Vander Vusse. Adaptation and overtraining in horses subjected to increasing training loads. / Appl Physiol 76: 1908-1913 1994.

8. Chaouloff $\mathrm{F}$. Physiopharmacological interactions between stress hormones and central serotonergic systems. Brain Res Brain Res Rev 18: 1-32. 1993.

9. Clement D.B., R.C. Asmundson, C.W. Medhurst. Hemoglobin values: comparative survey of the 1976 Canadian Olympic team. Can Med Assoc J 117: 614-616. 1977.

10. Cluydts R. Sleep and Mood. Doctoral Dissertation. University of Brussels, Brussels, 1979

11. Durnin J.V.G.A., J. Womersley. Body fat assessed from total body density and its estimation from skinfold thickness: measurement on 481 men and women aged from 16 to 72 year. Brit. J. Nutr. 32: 77-97 1974.

12. Fitzgerald L. Overtraining increases the susceptibility to infection. Int / Sports Med 12 Suppl 1: S5-8. 1991.

13. Foster $C$. Monitoring training in athletes with reference to overtraining syndrome. Med Sci Sports Exerc 30: 1164-1168 1998.

14. Fry R.W., A.R. Morton, G.P. Crawford, D. Keast. Cell numbers and in vitro responses of leucocytes and lymphocyte subpopulations following maximal exercise and interval training sessions of different intensities. Eur / Appl Physiol Occup Physiol 64: 218-227 1992.

15. Fry R.W., A.R. Morton, D. Keast. Overtraining in athletes. An update. Sports Med 12: 32-65. 1991.

16. Fry R.W., A.R. Morton, D. Keast. Acute intensive interval training and T-lymphocyte function. Med Sci Sports Exerc 24: 339-345. 1992.

17. Gabriel H.H., A. Urhausen, G. Valet, U. Heidelbach, W. Kindermann. Overtraining and immune system: a prospective longitudinal study in endurance athletes.

Med Sci Sports Exerc 30: 1151-1157 1998. 
18. Gastmann U., M. Lehmann. Monitoring overload and regeneration in cyclists. In: Overload, Performance Incompentence and Regeneration in Sport, edited by M. Lehmann, C. Foster, U. Gastmann, H. A. Keizer and J. M. Steinacker. New York: Kluwer Academic/Pleum Publishers, 1999, p. 131-148.

19. Guglielmini C., I. Casoni, M. Patracchini, F. Manfredini, G. Grazzi, M. Ferrari, $\mathrm{F}$. Conconi. Reduction of $\mathrm{Hb}$ levels during the racing season in nonsideropenic professional cyclists. Int J Sports Med 10: 352-356. 1989.

20. Harro J., H. Rimm, M. Harro, M. Grauberg, K. Karelson, A.M. Viru. Association of depressiveness with blunted growth hormone response to maximal physical exercise in young healthy men. Psychoneuroendocrinology 24: 505-517. 1999.

21. Jeukendrup A., W. Saris, F. Brouns, K. ADM. A new validated endurance performance test. Medicine and Science in Sports and Exercise 28: 266-270 1996.

22. Kuipers H., F.T.J. Verstappen, H.A. Keizer, P. Geurten, G. Kranenburg van. Variability of Aerobic Performance in the Laboratory and Its Physiologic Correlates. Sport Med 6: 197-201 1985.

23. Lehmann M., P. Baumgartl, C. Wiesenack, A. Seidel, H. Baumann, S. Fischer, U. Spori, G. Gendrisch, R. Kaminski, J. Keul. Training-overtraining: influence of a defined increase in training volume vs training intensity on performance, catecholamines and some metabolic parameters in experienced middle- and long-distance runners. Eur J Appl Physiol Occup Physiol 64: 169-177 1992.

24. Lehmann M., H.H. Dickhuth, C. Gendrisch, W. Lazar, M. Thum, R. Kaminski, J.F. Aramendi, E. Peterke, W. Wieland, J. Keul. Training-overtraining. A prospective, experimental study with experienced middle- and long-distance runners. Int / Sports Med 12: 444-452. 1991.

25. Lehmann M., C. Foster, J. Keul. Overtraining in endurance athletes: a brief review. Med Sci Sports Exerc 25: 854-862 1993.

26. Lehmann M., U. Gastmann, K.G. Petersen, N. Bachl, A. Seidel, A.N. Khalaf, S. Fischer, J. Keul. Training-overtraining: performance, and hormone levels, after a defined increase in training volume versus intensity in experienced middle- and long-distance runners. Br / Sports Med 26: 233-242. 1992.

27. Lehmann M., K. Knizia, U. Gastmann, K.G. Petersen, A.N. Khalaf, S. Bauer, L. Kerp, J. Keul. Influence of 6-week, 6 days per week, training on pituitary function in recreational athletes. Br / Sports Med 27: 186-192 1993.

28. Lehmann M. H. Mann, U. Gastmann, J. Keul, D. Vetter, J.M. Steinacker, D. Haussinger. Unaccustomed high-mileage vs intensity training-related changes in performance and serum amino acid levels. Int / Sports Med 17: 187.192 1996.

29. Mackinnon L.T. S.L. Hooper, S. Jones, R.D. Gordon, A.W. Bachmann. Hormonal, immunological, and hematological responses to intensified training in elite swimmers. Med Sci Sports Exerc 29: 1637-1645. 1997.

30. Miller J. Discrete versus continuous stage models of human information processing in search of partial output. J Exp Psychol Hum Percept Perform \&: 273-296. 1982.

31. Morgan W.P. Affective beneficence of vigorous physical activity. Med Sci Sports Exerc 17: 94-100. 1985 .

32. Morgan W.P. D.R. Brown, J.S. Raglin, PJ. O'Connor, K.A. Ellickson. Psychological monitoring of overtraining and staleness. Br J Sports Med 21: 107-114 1987. 
33. Morgan W.P. D.L. Costill, M.G. Flynn, I.S. Raglin, PJ. O'Connor. Mood disturbance following increased training in swimmers. Med Sci Sports Exerc 20: $408-4141988$.

34. O'Connor P.. W.P. Morgan, J.S. Raglin. Psychobiologic effects of $3 \mathrm{~d}$ of increased training in female and male swimmers. Med Sci Sports Exerc 23: 1055-1061 1991.

35. Peino R., A. Leal, R.V. Garcia-Mayor, F. Cordido, D. Micic, H. Kopeschaar, C. Dieguez, F.F. Casanueva. The use of growth hormone (GH) secretagogues in the diagnosis of $\mathrm{GH}$ deficiency in humans. Growth Horm IGF Res 9 Suppl A: 101. 105. 1999.

36. Raglin J.S., W.P. Morgan, PJ. O'Connor. Changes in mood states during training in female and male college swimmers. Int / Sports Med 12: 585-589 1991.

37. Rietjens G., H. Kuipers, F. Hartgens, H.A. Keizer. Red blood cell profile of elite Olympic triathletes. A thee-year follow up. Int / Sport med accepted for publication 2002.

38. Rowbottom D.G., D. Keast, C. Goodman, A.R. Morton. The haematological, biochemical and immunological profile of athletes suffering from the overtraining syndrome. Eur J Appl Physiol Occup Physiol 70: 502-509 1995.

39. Thorner M., M. Vance, E. Horvath, K. Kovacs. The Anterior Pituitary. In: Williams Textbook of Endocrinology (8th Edition ed.). Philadelphia: W.B. Saunders Company, 1992, p. 249-341.

40. Verde T., S. Thomas, R.J. Shephard. Potential markers of heavy training in highly trained distance runners. Br / Sports Med 26: 167-175. 1992.

41. Viru A., K. Karelson, T. Smirnova. Stability and variability in hormonal responses to prolonged exercise. Int / Sports Med 13: 230-235 1992. 



\section{CHAPTER ?}

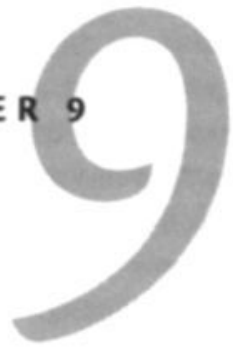

Physiological and psychological variables respond different in competitive cyclists and runners after a period of intensified training

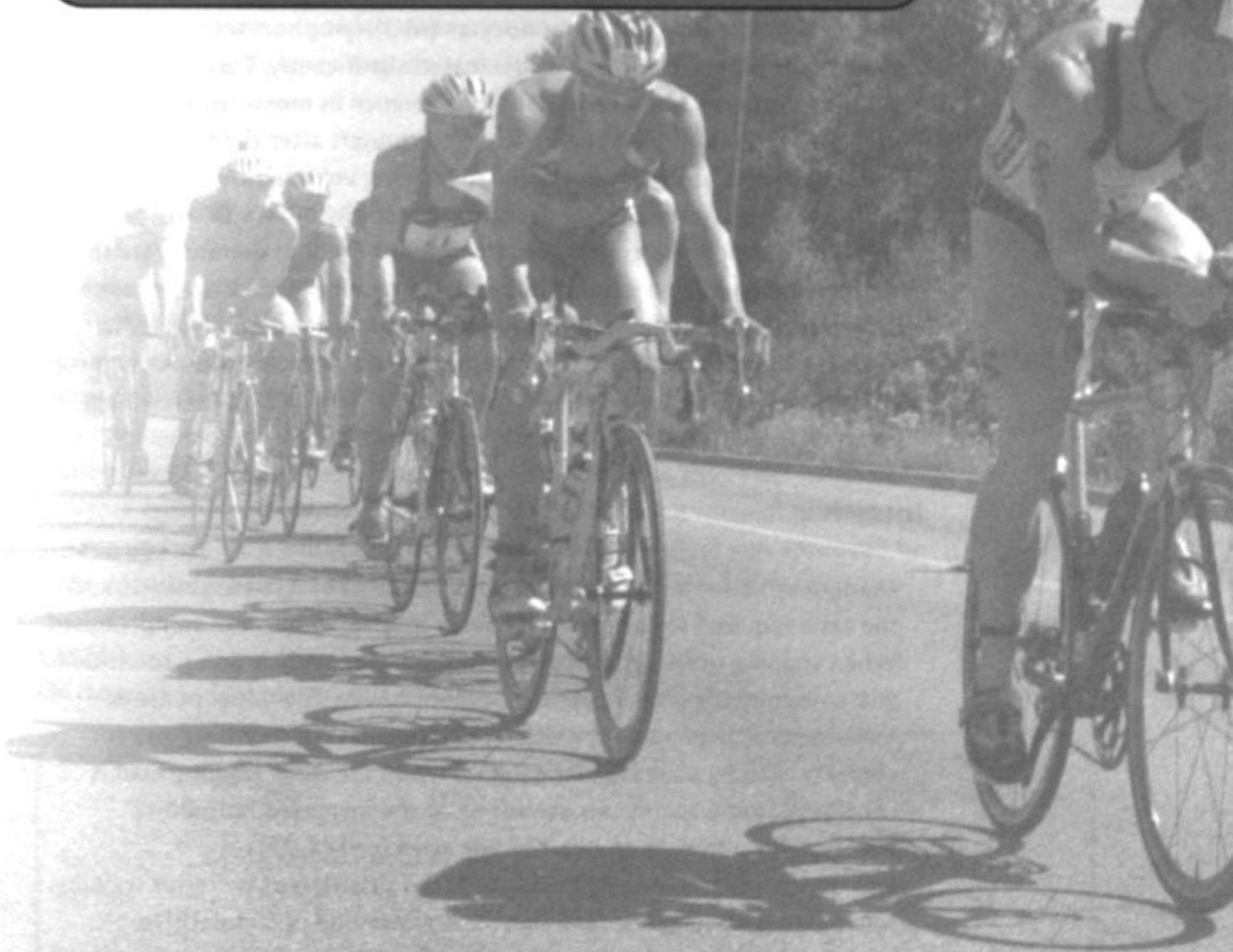

G.J.W.M. Rietjens, H. Kuipers, R.W. Klomp, A. Koops and H.A. Keizer. 


\section{Abstract}

The purpose of this study was to compare blood chemical, neuroendocrie, performance and psychological parameters in well-trained cyclists and i runners after two weeks of intensified training. Four healthy, competitiv runners (age $27 \pm 6$ years (mean $\pm \mathrm{SD}$ ), $\mathrm{VO}_{2} \max 65.8 \pm 5.2 \mathrm{ml} \cdot \mathrm{kg}^{\prime} \cdot \mathrm{min}^{-1}$ ) and 5 competitive cyclists (age $25 \pm 5$ years, $\mathrm{VO}_{2} \max 63.8 \pm 6.3 \mathrm{ml} \cdot \mathrm{kg}^{\prime} \cdot \mathrm{mi}^{\prime}$ ) participated in this study. Doubling of the normal training volume durin two weeks resulted in a significant increase in training load $(P<0.01)$, training strain $(P<0.01)$ and monotony $(P<0.01)$ in both groups. Howeve runners showed a higher increase in training load and strain. In line with this the rate of perceived exertion (RPE) increased significantly $(P<0.05)$ however there was no difference between the two groups. Maximal wor: load and maximal oxygen uptake did not change over the time in runne: and cyclist. No differences were found for (sub) maximal heart rate and lactate values within the two groups. Hemoglobin $(\mathrm{Hb})$, hematocrit $(\mathrm{Hct}$ and red blood count (RBC), monocytes (MO), lymphocytes (LY) and percentage monocytes (MOPR) decreased significantly. Cyclists and runners showed a similar pattern. No difference in measured endocrine

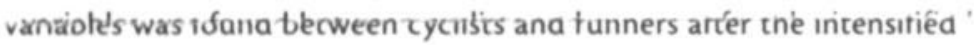
training period. However the doubled training volume resulted in both groups in a significant cortisol decrease after stimulation with corticotropinreleasing hormone. In conclusion, the present findings demonstrate that runners and cyclists do respond differently in training load, -strain, -monotony, POMS and performance after two weeks of intensified training. Other measured variables did not show any difference between these two groups of endurance athletes.

\section{Introduction}

Modern athletic training requires large amounts of strenuous work, and the time required for adequate recovery may easily become compromised. When training outweighs recovery, an athlete actually is doing too much and consequently is overtraining unintentionally. The initial phase of unintentional overtraining is often referred to as overreaching and is characterized by increased fatigability. When the same training load is continued, more symptoms are added to the increased fatigability, eventually resulting in an overtraining syndrome $(8,12)$.

Endurance athletes are all familiar with the situation of excessive training. associated with long lasting fatigue and worsening of competitive performance. The natural response is to further increase the training load in an attempt to improve physical condition and usually this results in 
further deterioration of performance (22). To tune the training and avoid overtraining both physiological and psychological markers have been used. Various hematological, blood chemical and psychological variables have been suggested as indicator for detecting early overtraining $(16,20)$.

Unfortunately all attempts to identify a reliable, specific and sensitive parameter for early staleness, have failed so far. Barron et al (1985) reported in four overtrained athletes changes in the hypothalamic-pituitary axis (HPA) (2). Although overtraining is known in most sports, possible sport related differences in susceptibility and symptoms may exist. No data exist whether the susceptibility to become overtrained is different between various sports. Most of the overtraining studies investigated the effects of an intensified training period in one kind of sport, which makes comparison difficult. Differences in the type of training between several sports may influence several factors associated with training (mal) adaptation.

Therefore we investigated possible differences in response to an intensified training period in runners and cyclists. For this purpose a selected number of blood biochemical, neuro-endocrine and psychological parameters were studied in long distance runners and cyclists after two weeks of intensified training.

\section{Materials and methods}

\section{Subjects}

A total of nine healthy, well-trained, competitive male endurance athletes ( 4 runners, 5 cyclists) with a training experience of at least 4 years and a maximal oxygen uptake of at least $55 \mathrm{ml} \cdot \mathrm{kg} \cdot \mathrm{min}$ participated in this study. Physiological characteristics of the subjects are listed in Table 1. None of them was taking any medication known to affect the HPA axis.

Before entering the study the subjects had to fill out a medical questionnaire and signed a written informed consent. The project was approved by the Medical Ethical Committee of the Maastricht University.

\section{TABLE 1.}

Characteristics of the runners and cyclists

\begin{tabular}{|lccccc|}
\hline Subject & $\begin{array}{c}\text { Age } \\
\text { (years) }\end{array}$ & $\begin{array}{c}\text { Height } \\
(\mathrm{cm})\end{array}$ & $\begin{array}{c}\text { Body mass } \\
(\mathbf{k g})\end{array}$ & $\begin{array}{c}\text { Body fat } \\
(\%)\end{array}$ & $\begin{array}{c}\mathrm{VO}_{2} \mathrm{max}^{\prime} \\
\left(\mathrm{ml}^{\prime} \cdot \mathrm{kg}^{\prime} \cdot \mathrm{min}^{\prime}\right)\end{array}$ \\
Runners & $27.3 \pm 6$ & $181 \pm 7$ & $67.6 \pm 7.6$ & $10.3 \pm 1.1$ & $65.8 \pm 5.2$ \\
Cyclists & $25.2 \pm 5$ & $184 \pm 2.4$ & $74.5 \pm 6.8$ & $12.7 \pm 2.1$ & $63.8 \pm 6.3$ \\
Total & $24.6 \pm 4$ & $182 \pm 4.7$ & $71.4 \pm 7.6$ & $11.6 \pm 2$ & $64.7 \pm 5.6$ \\
\hline
\end{tabular}

Values are presented as mean \pm SD 


\section{General design}

The subjects had to follow an intensified two week training program, seven days per week. Before entering this intervention period, subjects' normal training pattern was assessed over a period of two weeks (pre intervention period). Starting at day one of the pre intervention period, and continuing during the two week intervention period the subjects kept a training logbook in which they had to fill in their training time ( $\mathrm{min}$ ), and the rate of perceived exertion (RPE) of the training on a 1 to 10 scale of Borg ( 3 ) with $1=$ very very low and $10=$ very very heavy. From this weekly training load, training strain and training monotony were calculated according to Foster (7). Furthermore an adjusted Profile of Mood State (POMS) was administered daily throughout the pre- and intervention period (4). Based on the average daily training time over the two weeks pre intervention period, a training schedule for the intervention period was developed. The training volume was doubled (200\%) during the intervention period, while the training intensity was kept constant. Recovery days were eliminated from the training program and the total amount of training hours per week was spread over the seven days of the week. To control subjects' training intensity, in all subjects' heart rates were registered during all training of the pre intervention and intervention period (Polar, Kempele, Finland). In addition the subjects were followed during a taper period of two weeks. During this period, training volume was reduced to $50 \%$ of their normal training and at least two days of rest were added to the training schedule. Before and after the two weeks intensified training, subjects underwent several measurements as described further on. Furthermore, after the first and the second week of the taper period a performance test was done. To ensure that the conditions remain comparable, each subject was tested at the same time of the day before and after the intervention period. The cyclists underwent all performance tests on an electrically braked cycle ergometer (Lode Excalibur Sport, Lode, Groningen, The Netherlands), whereas the runners did all the exercise testing on a motor driven treadmill (Medifit, Maarn, The Netherlands). Before and after exercise, blood was drawn $(5 \mathrm{ml})$ from a catheter inserted in an antecubital vein, to assay lactate, growth hormone (GH), insuline-like growth factor 1 (IGF-1), adreno-corticotropic hormone (ACTH) and cortisol. Furthermore, before and after the intervention period a blood sample for hematology was drawn before the start of exercise. The following hematological variables were determined; Hemoglobin $(\mathrm{Hb})$, hematocrit ( $\mathrm{HCt}$ ), red blood cell count (RBC), Mean corpuscular hemoglobin $(\mathrm{MCH})$, Mean corpuscular hemoglobin content (MCHC), Mean corpuscular volume (MCV), white blood cell count (WBC), leucocytes, monocytes and granulocytes. 
Pre-intervention Intervention period Taper/after care

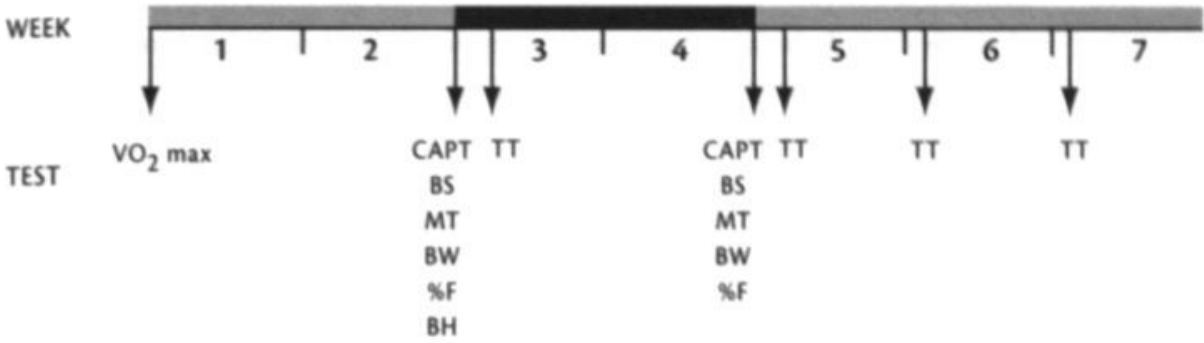

\section{FIGURE 1}

Time schedule of study. $\mathrm{VO}_{2}$ max = determination of the maximum oxygen uptake;

CAPT = combined anterior pituitary test; $B S=$ blood sampling $M T=$ maximal exercise test; $\mathrm{BW}=$ body weight, $\% \mathrm{~F}=$ percentage body fat; $\mathrm{BH}=$ body height;

$\mathrm{TT}=$ time trial

\section{Anthropometric parameters}

Body mass and percentage body fat were measured prior to and after the intervention period. Body height was only measured prior to the intervention period. Percentage body fat was estimated using the skin fold method, taking the sum of 4 skin folds (biceps, triceps, sub scapular and suprailiacal), measured in duplicate and applying the equation from Durnin and Womersley (5).

\section{Performance tests}

Cycling

Maximal exercise test $\left(M T_{b}\right)$.

After a warm up period of $5 \mathrm{~min}$ at $1 \mathrm{~W} \cdot \mathrm{kg}$ ' body mass, the workload was increased every 3 minutes by $0.5 \mathrm{~W} \cdot \mathrm{kg}^{\prime}$ body mass until a volitional exhaustion occurred. The maximal workload (Wmax) was defined as the last completed work load (W) plus the fraction of time spent in the final work load multiplied by the increment in workload (in Watts) (13). During the test expiratory gases were measured using an Oxycon $B$ ( $E$. Jaeger $\mathrm{GmbH}$, Höchberg, Germany). Along with these measurements the heart rate was monitored (Polar Sporttester, Kempele, Finland) at time intervals of 15 seconds.

\section{Time Trial $\left(T T_{b}\right)$}

Because it has been shown that a simulated time trial gives better reproducibility and reliability than the traditional incremental exercise tests (10), this latter test was used in order to determine subjects' maximal performance. The measure of performance was the time to complete an amount of work determined by the following formula:

Target amount of work $(J)=75 \% W \max \cdot 1800 \mathrm{sec}$ 
The Wmax in this formula was derived from the maximal exercise test described early on. During this test the subjects were not informed about their workload, pedalling rate, heart rate or elapsed time. To ensure comparable conditions, each subject was tested after a rest day at the same time of the day.

\section{Running.}

Maximal exercise test ( $M T_{\text {, }}$ )

After a warm up period of 5 minutes at $13.5 \mathrm{~km} / \mathrm{hrs}^{-1}$, speed was increased every 3 minutes by $2 \mathrm{~km} / \mathrm{hrs}^{-1}$ until $17.5 \mathrm{~km} / \mathrm{hrs}^{-1}$, where after the running speed was increased every $3 \mathrm{~min}$ by $1 \mathrm{~km} / \mathrm{h}$ until exhaustion. The Vmax was defined as the last completed workload $(\mathrm{km} / \mathrm{h})$ plus the fraction of time spent at the final work rate multiplied by the final increment in speed (13). During the test expiratory gases were measured using an Oxycon B (E. Jaeger $\mathrm{GmbH}$, Höchberg, Germany). Along with these measurements the heart rate was monitored (Polar Sporttester, Kempele, Finland) at 15 seconds time intervals.

\section{Time Trial (TT,)}

The TT, consisted of a run on a treadmill at a fixed pace of $90 \%$ of the calculated maximal speed (Vmax) attained at the MT, until volitional exhaustion. After a warming up at a pace of $60 \%$ of $V \max$ for 6 min the speed was increased to $90 \%$ of the Vmax. During the TT the runners were not informed about elapsed time and heart rate. For maximal performance measurement, time to exhaustion was used.

\section{Combined Anterior Pituitary Test}

The challenge test, in clinical settings also known as Combined Anterior Pituitary Test (CAPT), was used for assessing pituitary function (23). The CAPT took place before the maximal exercise test at the same day. The subjects were reported to the laboratory at $8.30 \mathrm{a} . \mathrm{m}$. after an overnight fast. Ten minutes after insertion of a Teflon catheter in an antecubital vein, a bolus injection with a physiological dose of releasing hormones in $10 \mathrm{ml}$ saline ( $33.3 \mu \mathrm{g}$ Corticotropin-releasing hormone (CRH) and $17 \mu \mathrm{g}$ Growth hormone releasing hormone (GhRH)) were administered. Blood $(10 \mathrm{ml})$ for analysis of $\mathrm{GH}, \mathrm{ACTH}$ and cortisol were sampled directly before the bolus injection, and 10, 20, 40, 60, 80, 100 minutes after the bolus injection. 


\section{Sample processing and analysis}

Four $\mathrm{ml}$ of blood for cortisol, GH and IGF-1 detection was collected in serum tubes and allowed to clot at room temperature and thereafter centrifuged at $5.000 \mathrm{rpm}$ at $4^{\circ} \mathrm{C}$. The resulting serum was collected and stored immediately at $-80^{\circ} \mathrm{C}$. Samples for $\mathrm{ACTH}$ were collected in pre cooled EDTA tubes and immediately centrifuged at $5000 \mathrm{rpm}$ at $4^{\circ} \mathrm{C}$. Plasma for $\mathrm{ACTH}$ was stored at $-20^{\circ} \mathrm{C}$. All samples from one subject were analysed in the same run to minimize inter-assay variability. Sequential Immunometric Assays were used to determine blood concentrations of cortisol (Chiron Diagnostics Corporation, East Walpole, MA, USA), GH (Nichos Institute Diagnostics San Juan Capistrano, CA, USA) and ACTH (Euro/DPC, United Kingdom). Variances for the chemiluminescence assays performed in this investigation were as follows: cortisol (intra-assay $4.5 \%$, inter-assy $6.4 \%$ ), GH (intra-assay $4.0 \%$, inter-assy $9.2 \%$ ), and ACTH (intra-assay $5.9 \%$, inter-assy $3.9 \%$ ). Quantification of IGF1 was performed

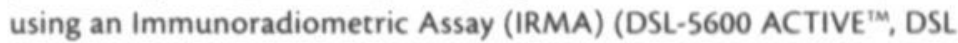
Deutschland $\mathrm{GmbH}$, Germany) with an intra-assay variance of $3.0 \%$ and an inter-assay of $1.5 \%$. Red and white blood cell samples $(4 \mathrm{ml})$ were collected in EDTA tubes and analysed immediately (Coulter MD18, Miami, USA).

\section{Profile of Mood State (POMS)}

An adjusted version of the POMS questionnaire (4) was administered daily during the pre intervention period and during the two weeks intervention period in order to quantify the influence of intensified training on mood state. The adjusted POMS assesses tension, depression, anger, vigor and fatigue. The total mood score was measured by summing the scores of the negative moods (tension, depression, anger and fatigue) and subtracting the vigor score. From this daily score a weekly average score was calculated. The higher the mood score, the worse the mood. Furthermore the average weekly score of each item separately was measured.

\section{Statistical analyzes}

All data are presented as means and standard deviation (SD). All individual parameters were tested for significance using a Wilcoxon signed ranks test for related pairs or the Wilcoxon, Mann-Whitney test for unrelated data. Time trial data were analyzed by two-way ANOVA with time as repeated measure. Scheffé's post-hoc test comparison were used to determine significant differences among the four test moments. An alpha level of $P<0.05$ was defined for all tests of significance. 


\section{Results}

\section{Antropometric data}

For the total group body mass (71.4 $\pm 7.6 \mathrm{vs} .71 .4 \pm 7.0 \mathrm{~kg}$ ) and body fat $(11.6 \pm 2.0$ vs. $11.6 \pm 2.0 \%$ ) did not change during the course of this study. There were no differences between runners and cyclists in the pre and post body mass and body fat data.

\section{Training}

All subjects successfully completed the two weeks of intensified training. As shown in table 2, the average amount of training minutes during the two monitoring weeks was $480 \pm 186 \mathrm{~min}$ and $435 \pm 91 \mathrm{~min}$ for cyclists and runners, respectively. During the next two weeks the subjects doubled the training volume ( $888 \pm 294 \mathrm{~min}$ and $1050 \pm 270 \mathrm{~min}$ for week 1 and week 2 , respectively in cyclists and $773 \pm 66 \mathrm{~min}$ during the first week and $1075 \pm 92$ min during the second week in runners) compared to the base training. This increase in training volume resulted in a significant increase in the individual training load ( $120 \%$ increase, $P<0.001)$, training strain ( $380 \%$ increase, $P<0.001)$, and training monotony ( $110 \%$ increase, $P<0.001)$ in cyclists. Runners also significantly increased their training load $(215 \%$ increase, $P<0.001)$, training strain $(615 \%$ increase, $P<0.001$ ) and training monotony ( $130 \%$ increase, $\mathrm{P}<0.001)$.

\section{Performance tests}

\section{Maximal exercise test}

Both the cyclists and the runners showed a small increase in their maximal workload after the intensified training period $(3.2 \%$ and $3.1 \%$, respectively; $P>0.05$ ). However, this increase was not significant. Maximal heart rates registered during the tests were significantly $(P<0.05)$ reduced after the period of intensified training, but no significant changes on the peak physiological values of maximal lactate and $\mathrm{VO}_{2}$ max were found for both the cyclists and runners. Sub-maximal heart rates and lactate values showed no changes. No difference between the runners and cyclists in any of these (sub) maximal variables was observed.

\section{Time Trials}

The cyclists showed a significant increase in time to complete the preset amount of work (increase of $3.2 \% ; P<0.01$ ) after the two weeks of intensified training (from $29.1 \pm 1.4 \mathrm{vs} .30 .1 \pm 1.8 \mathrm{~min}$ ). One week tapering resulted in a significant performance improvement (decrease of $4.1 \%$ in time to compete the time trial $\mathrm{P}<0.05$ ). After the second taper week performance returned to pre test values. In contrast to the cyclists, in the runners neither the intensified training, nor the tapering had a significant 
influence on the time to exhaustion. In contrast to the cyclists the runners showed a small, non-significant improvement $(+4.1 \%)$ in performance (time to exhaustion) after the intensified training period. However, after one week tapering the mean time to exhaustion decreased to pretest values.

Due to illness of three of the runners no data were available after two weeks of tapering.

\section{Hormones}

No significant changes in resting and maximal values of $\mathrm{GH}$, Cortisol and $\mathrm{ACTH}$, obtained during the maximal exercise test before and after the intervention period, were found within the total group and between the groups.

\section{The Combined Anterior Pituitary Test}

The mean plasma concentrations of $\mathrm{CH}, \mathrm{IGF}-1$ and ACTH obtained during the CAPT tended to be decreased, however not significant. Cortisol values measured during the second CAPT were significantly lower. No significant differences in pre and post hormone values were detected between the two groups.

\section{Hematological variables}

White blood cell profile.

An overview of the white blood cell parameters of all subjects is presented in table 2. Due to technical problems the data of one cyclist were lost.

All values were within the normal range for all subjects. The total number of leukocytes tended to decrease, after the intensified training. Despite a lack of a significant decrease in the WBC, two subpopulation of the leukocytes, monocytes $(\mathrm{MO})$ and lymphocytes $(\mathrm{LY})$, and the percentage monocytes (MOPR) decreased significantly $(P<0.017 ; P<0.043$ and $P<0.025$, respectively). No significant changes were seen between the cyclists and runners in the white blood cell parameters as presented in table 2. 
TABLE 2

Mean hematological values

\begin{tabular}{|c|c|c|c|c|c|c|}
\hline & \multicolumn{2}{|c|}{ Pre-intervention } & \multicolumn{2}{|c|}{ Post-intervention } & \multicolumn{2}{|c|}{$\begin{array}{l}\text { Delta pre-/post- } \\
\text { intervention }\end{array}$} \\
\hline & CYCLISTS & RUNNERS & CYCLISTS & RUNNERS & $\begin{array}{l}\text { DELTA } \\
\text { CYCLISTS }\end{array}$ & $\begin{array}{c}\text { DELTA } \\
\text { RUNNERS }\end{array}$ \\
\hline $\operatorname{RBC}\left(10^{12}\right)$ & $4.67 \pm 0.29$ & $5.01 \pm 0.15$ & $4.45 \pm 0.28$ & $4.83 \pm 0.13$ & $0.22 \pm 0.18$ & $0.18 \pm 0.15$ \\
\hline $\mathrm{Hb}(\mathrm{mmol} / \mathrm{l})$ & $8.92 \pm 0.41$ & $9.10 \pm 0.35$ & $8.48 \pm 0.33$ & $8.85 \pm 0.33$ & $0.44 \pm 0.34$ & $0.25 \pm 0.21$ \\
\hline $\operatorname{Hct}(\mathrm{I} / \mathrm{I})$ & $0.45 \pm 0.02$ & $0.45 \pm 0.01$ & $0.40 \pm 0.03$ & $0.43 \pm 0.01$ & $0.05 \pm 0.03$ & $0.02 \pm 0.01$ \\
\hline MCV (fl) & $95.54 \pm 5.08$ & $88.80 \pm 1.00$ & $89.16 \pm 2.85$ & $88.78 \pm 1.13$ & $6.38 \pm 5.05$ & $0.03 \pm 1.61$ \\
\hline $\mathrm{MCH}$ (fmol) & $1.92 \pm 0.04$ & $1.83 \pm 0.02$ & $1.90 \pm 0.08$ & $1.84 \pm 0.03$ & $0.02 \pm 0.09$ & $-0.03 \pm 0.05$ \\
\hline MCHC (mmol/l) & $20.08 \pm 1.01$ & $20.45 \pm 0.33$ & $21.36 \pm 0.40$ & $20.70 \pm 0.32$ & $-1.20 \pm 1.33$ & $-0.25 \pm 0.29$ \\
\hline RDW (\%) & $12.88 \pm 0.38$ & $14.70 \pm 4.37$ & $13.02 \pm 0.41$ & $13.08 \pm 0.43$ & $-0.14 \pm 0.29$ & $1.63 \pm 4.21$ \\
\hline WBC $\left(10^{9} / 1\right)$ & $5.33 \pm 1.68$ & $6.83 \pm 0.74$ & $6.05 \pm 2.13$ & $5.63 \pm 1.03$ & $-0.73 \pm 2.60$ & $1.20 \pm 1.63$ \\
\hline LYPR (\%) & $38.63 \pm 1.55$ & $29.45 \pm 8.20$ & $35.63 \pm 7.17$ & $34.70 \pm 8.68$ & $3.00 \pm 7.29$ & $-5.20 \pm 6.87$ \\
\hline MOPR (\%) & $23.75 \pm 13.33$ & $8.25 \pm 3.80$ & $6.80 \pm 1.76$ & $5.30 \pm 3.69$ & $16.90 \pm 15.00$ & $2.95 \pm 1.99$ \\
\hline GRPR (\%) & $37.63 \pm 13.87$ & $62.30 \pm 10.43$ & $57.58 \pm 8.05$ & $60.00 \pm 12.00$ & $-19.95 \pm 20.78$ & $2.30 \pm 8.39$ \\
\hline $\operatorname{tr}\left(10^{9} / 1\right)$ & $2.08 \pm 0.67$ & $1.98 \pm 0.43$ & $1.78 \pm 0.62$ & $1.90 \pm 0.29$ & $0.30 \pm 0.20$ & $0.08 \pm 0.15$ \\
\hline $\mathrm{MO}(10 \% / 1)$ & $1.30 \pm 0.85$ & $0.58 \pm 0.17$ & $0.35 \pm 0.06$ & $0.25 \pm 0.13$ & $0.95 \pm 0.87$ & $0.33 \pm 0.09$ \\
\hline GR( $\left(10^{\circ} / 1\right)$ & $1.95 \pm 0.75$ & $4.30 \pm 1.09$ & $2.83 \pm 0.91$ & $3.45 \pm 1.29$ & $0.51 \pm 1.38$ & $0.86 \pm 1.28$ \\
\hline
\end{tabular}

Hematological values of the cyclists $(n=5)$ and runners $(n=4)$ as measured before and after the two weeks of intensified training. Values are given as mean $\pm S D$.

(RBC red blood cell count; $\mathrm{Hb}$ hemoglobine; Hct hematocrit; MCV mean corpuscular volume; $\mathrm{MCH}$ mean corpuscular hemoglobin; $\mathrm{MCHC}$ mean corpuscular hemoglobin concentration; RDW red cell distribution width; WBC white blood cel 1 count; LYPR percentage lymphocytes; MOPR percentage moncytes; GRPR percentage granulocytes; LY lymphocytes; MO moncytes; GR granulocytes.

\section{Red Blood cell profile}

All mean and individual values for red blood cell variables were within the established normal ranges for men. Of all measured red blood cell variables (table 2) RBC, hemoglobin $(\mathrm{Hb})$ and hematocrit $(\mathrm{Hct})$ showed a significant decrease 4,2\% ( $P<0.015), 4,0 \%(P<0.011)$ and $8,1 \%(P<0.021)$ respectively, after the two weeks of training. Mean corpuscular hemoglobin concentration (MCHC) increased significantly by $4.1 \%(P<0.038)$. When the two groups were compared none of the red blood cell parameters behaved different (Table 2). 


\section{Psychological variables}

POMS The average mood state score is presented in Fig 2 . The average mood score did increase significantly throughout the observation period $(37.3 \pm 7.7 ; 37.9 \pm 7.2 ; 39.6 \pm 9.9$ and $40.7 \pm 8.9$ for base line, week 1 and week 2 of the intensified training and the post intervention week, respectively).

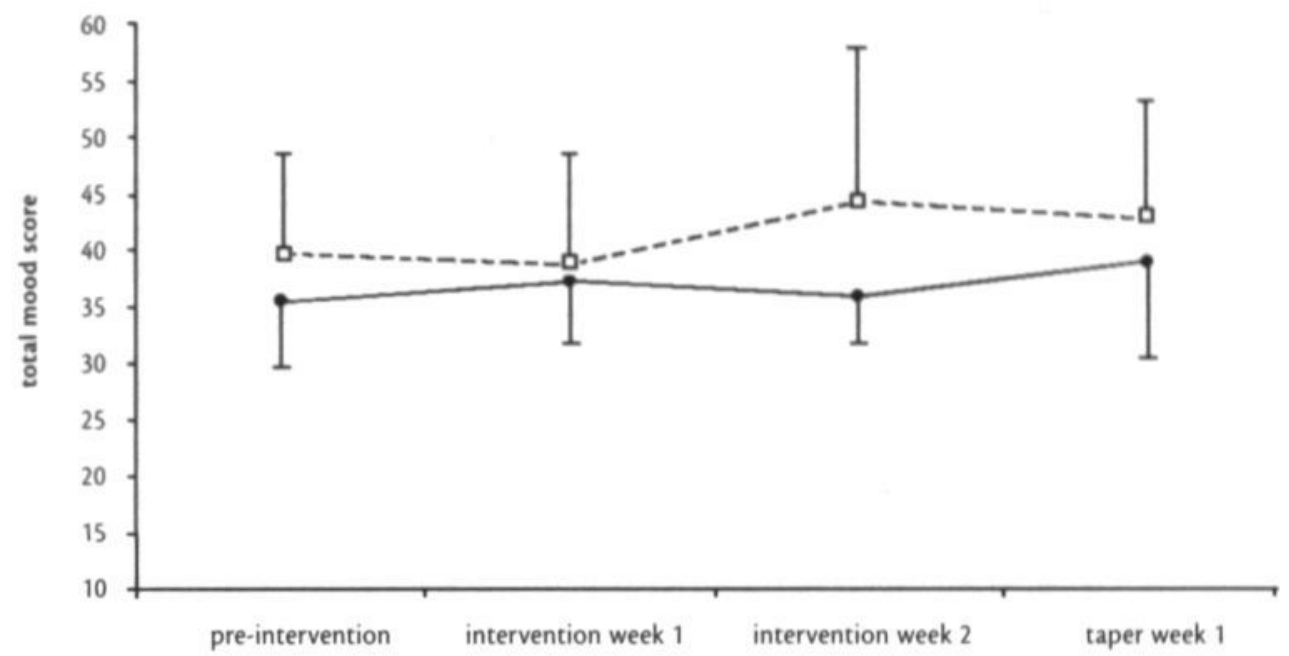

\section{FIGURE 2}

The average total mood score during the pre intervention period, intervention period and taper period in runners $(\square)$ and cyclists $(\bullet)$.

When analyzing the five sub scales of the POMS separately, only fatigue in the taper period increased significantly compared to the base line score $(P<0.05)$. This increase was mainly caused by the runners. Although not significant, the same trend was observed for vigor.

Between the runners and cyclists there were no significant differences in the average mood score and in the five sub scales of the POMS separately. However, as stated before the runners showed a noticeable increase in the fatigue score towards the end of the intensified training period and crossing the vigor score (Fig 3 ). 

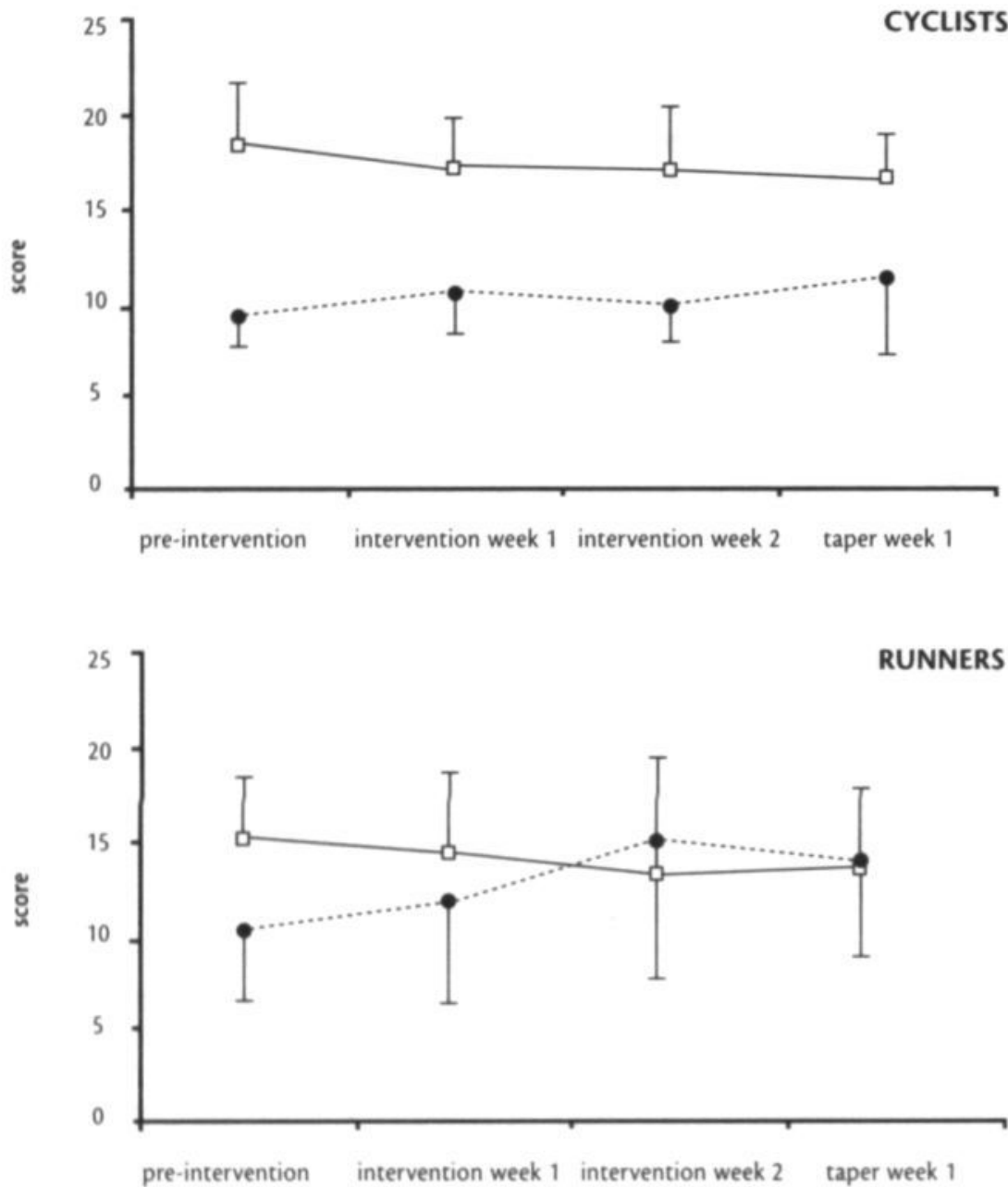

\section{FIGURE 3}

The average score of the sub items vigor $(\square)$ and the fatigue (-) in the cyclists and runners during the pre-intervention, intervention, an taper period. Note: Interestingly, in runners the vigor and fatigue lines crossed each others.

This phenomenon is often associated with the overtraining syndrome.

\section{Rating of perceived exertion}

The mean rating of perceived exertion (RPE) score increased significantly from $3.2 \pm 1.7$ in the pre training period to $5.3 \pm 1.5$ in the first week of the intensified training $(P<0.01)$ and $5.6 \pm 1.5$ during the second week of intensified training period $(P<0.01)$. During the tapering period the RPE score decreased. Both the runners and cyclists showed a similar patron of the RPE score during the intensified training and tapering. 


\section{Discussion}

The present study intended to investigate possible differences in physiological and psychological responses to increased training in runners compared to cyclists. For this purpose, both the runners and the cyclists doubled their training volume and all recovery days were skipped during a period of two weeks. This increase in training volume resulted in an increase in the calculated training load, training strain and training monotony in both groups. In the present study, the percentage increase in mean training load, strain and monotony was higher in runners $(215 \%$, $615 \%$ and $130 \%$ increase, respectively) compared to the cyclists ( $120 \%$, $380 \%$ and $110 \%$ increase, respectively). However the difference between the groups did not reach the level of significance $(P=0.06)$, which most probably is caused by the small number of subjects involved in the present study. This difference in training load, strain and monotony between runners and cyclists could not be found for the RPE scores.

Since the training load, strain and monotony are a result of the product of RPE and training time, a possible difference between runners and cyclists can not be attributed to the larger daily training time for cyclists, but rather might be caused by the type of sport (greater energy expenditure per unit time and/of the greater impact on muscle in case of the runners). Based on the results of this study it must be emphasized that changes in training load, strain and monotony can easily be misinterpreted because these variables can be the result of a change in training discipline rather than indicating a negative training adaptation. Therefore, in a multiple event sport like triathlon, the RPE seems a more appropriate tool in measuring training load.

The intensified training in the present study resulted in an increase in the total mood score for both groups. No differences were found between the two groups according to the total mood score. This result confirms the findings of Morgan et al. (1987) (16), who compared the POMS result of a group of wrestlers and swimmers and found similar results between both groups. From this, they concluded that the POMS can be used in different types of sport (16). However, analyzing the sub-scores in the present study separately, the runners showed a more pronounced increase in fatigue. The increased fatigue score crossed the vigor score and resulted in an inverted iceberg profile often seen in overtrained athletes and a decrease in performance (16). However, in the present study the runners increased their performance reflected in an increase in time to exhaustion.

This inconsistency, which was not found in the cyclists, may be attributable to the small number of runners $(n=4)$. However, it might also be possible that individual POMS scores are not as sensitive as for instance found in swimmers and rowers (17-19). 
Another interesting finding of this study was that runners showed different performance patterns over time compared to cyclists. Where the cyclists had a drop in performance after the two weeks of intensified training, the runners improved their performance. One week tapering showed the opposite result; the cyclists improved their performance above pre test level, but the runners decreased their performance to pre test values. In a similar experiment with runners (Keizer, unpublished data) we have observed an identical reaction pattern. To date we have no explanation for this phenomenon.

In the present study both groups showed a decrease in $\mathrm{RBC}, \mathrm{Hb}$ and $\mathrm{Hct}$ after the intensified training. In endurance athletes low RBC, $\mathrm{Hb}$ and $\mathrm{Hct}$ levels are often seen and referred to as 'sports anemia' $(24,25)$. One of the most commonly noted causes of sports anemia is based on the compression- and impact-related mechanical damage of the RBC's in the sole of the foot and it is obvious that this 'heel-strike' hemolysis is more common in runners than in cyclists $(1,6,21)$. The results of the present study, however, do not support this assumption as there was no difference between the runners and cyclists in the decrease in RBC, $\mathrm{Hb}$ and $\mathrm{Hct}$ after the intensified training period. To the best of our knowledge there are only two studies that investigated the differences between runners and cyclists concerning hematological variables. Green and co-workers investigated the hematology in well trained cyclists and runners and their findings parallel the findings of the present study (9). However Krebs (1992) found in runners significantly lower RBC and $\mathrm{Hct}$ values after a period of training compared to cyclists (11).

Of all hormones measured in this study, only one hormone was affected by the intensified training. After stimulating the HPA axis with $\mathrm{CRH}$ a significant decrease in the cortisol release was seen. This finding confirms the finding of Lehman et al. (1993) who found also a lower adrenal cortisol response after a period on intensified training (14). However, in that study CRH stimulation produced a $60 \%$ higher pituitary ACTH response. Probably because the shorter intensified training period of the present study (Lehman trained his subjects for six weeks) a compensatory pituitary ACTH response didn't appear (14). No differences in cortisol values were found between runners and cyclists. This in line with a finding of Lucia et al (1996) (15), who also failed to find any differences in cortisol and testosterone values between professional cyclists, elite triathletes and recreational marathon runners.

Taken together, the present study has shown that there are differences in response after a period of intensified training between runners and cyclists and that these differences occur mainly on the psychological variables (training load, strain and monotony, and POMS. Since our runners experienced severely deteriorated exercise performance and fatigue in the 
weeks and months after the intensified training, coaches must be aware of this unexplained phenomenon. Furthermore runners showed a different performance pattern compared to the cyclists as a reaction to the intensified training period and taper period. As these variables are often used as a tool for monitoring training status these results are especially interesting for those athletes who use a variety of training modes as for instance triathletes.

\section{Acknowledgement}

We thank the Netherlands Olympic Committee (NOC*NSF) for funding this work.

The authors express their gratitude to Dennis van Hamont for his assistance during all the testing and Dr. A. Kester for his statistical advice. 


\section{References}

1. Balaban E.P. Sports anemia. Clin Sports Med 11: 313-325. 1992.

2. Barron G.L., T.D. Noakes, W. Levy, C. Smith, R.P. Millar. Hypothalamic dysfunction in overtrained athletes. J Clin Endocronilogy and Metabolism 60: 803-806 1985.

3. Borg G.A. Psychophysical bases of perceived exertion. Med Sci Sports Exerc 14: 377-381 1982.

4. Cluydts R. Sleep and Mood. Doctoral Dissertation. University of Brussels, Brussels, 1979

5. Durnin J.V.G.A., J. Womersley. Body fat assessed from total body density and its estimation from skinfold thickness: measurement on $\mathbf{4 8 1}$ men and women aged from 16 to 72 year. Brit. J. Nutr. 32: 77-97 1974.

6. Eichner E.R. Runner's macrocytosis: a clue to footstrike hemolysis. Runner's anemia as a benefit versus runner's hemolysis as a detriment. Am J Med 78: 321 . 325. 1985.

7. Foster $\mathrm{C}$. Monitoring training in athletes with reference to overtraining syndrome Med Sci Sports Exerc 30: 1164-1168 1998.

8. Fry R.W., A.R. Morton, D. Keast. Overtraining in athletes. An update. Sports Med 12: 32-65. 1991.

9. Green H.J., S. Carter, S. Grant, R. Tupling, G. Coates, M. Ali. Vascular volumes and hematology in male and female runners and cyclists. Eur I Appl Physiol Occup Physiol 79: 244-250. 1999.

10. Jeukendrup A., W. Saris, F. Brouns, K. ADM. A new validated endurance performance test. Medicine and Science in Sports and Exercise 28: 266-270 1996.

11. Krebs P.S. The effects of cycling and marathon training on eighteen blood parameters. / Sports Med Phys Fitness 32: 64-69. 1992.

12. Kuipers H., H.A. Keizer. Overtraining in Elite Athletes, Review and directions for the Furture. Sports medicine 6: 79-92 1988.

13. Kuipers H., F.T.J. Verstappen, H.A. Keizer, P. Geurten, G. Kranenburg van. Variability of Aerobic Performance in the Laboratory and Its Physiologic Correlates. Sport Med 6: 197-201 1985.

14. Lehmann M., K. Knizia, U. Gastmann, K.G. Petersen, A.N. Khalaf, S. Bauer, L. Kerp, J. Keul. Influence of 6-week, 6 days per week, training on pituitary function in recreational athletes. Br J Sports Med 27: 186-192 1993.

15. Lucia A., J.L. Chicharro, M. Perez, L. Serratosa, F. Bandres, J.C. Legido. Reproductive function in male endurance athletes: sperm analysis and hormonal profile. J Appl Physiol 81: 2627-2636. 1996.

16. Morgan W.P., D.R. Brown, J.S. Raglin, PJ. O'Connor, K.A. Ellickson. Psychological monitoring of overtraining and staleness. Br / Sports Med 21: 107-114 1987.

17. Morgan W.P., D.L. Costill, M.G. Flynn, J.S. Raglin, P.J. O'Connor. Mood disturbance following increased training in swimmers. Med Sci Sports Exerc 20: 408-414 1988.

18. O'Connor P... W.P. Morgan, J.S. Raglin. Psychobiologic effects of $3 \mathrm{~d}$ of increased training in female and male swimmers. Med Sci Sports Exerc 23: 1055-1061 1991.

19. Raglin J.S. W.P. Morgan. Development of a scale for use in monitoring traininginduced distress in athletes. Int / Sports Med 15: 84-88 1994. 
20. Rowbottom D.C. D. Keast, C. Goodman, A.R. Morton. The haematological, biochemical and immunological profile of athletes suffering from the overtraining syndrome. Eur J Appl Physiol Occup Physiol 70: 502-509 1995.

21. Seiler D. D. Nagel, H. Franz, P. Hellstern, C. Leitzmann, K. Jung. Effects of longdistance running on iron metabolism and hematological parameters. Int J Sports Med 10: 357-362. 1989.

22. Verde T., S. Thomas, R.J. Shephard. Potential markers of heavy training in highly trained distance runners. Br J Sports Med 26: 167-175. 1992.

23. Williams W.J., D.A. Nelson, M.W. Morris. Examination of the blood. In: Hematology, edited by W. J. Williams, E. Beutler, A. J. Erslev and M. A. Lichtman. New York: McGraw-Hill, 1990.

24. Yoshimura H. Anemia during physical training (sports anemia), Nutr Rev 28: 251 . 253. 1970.

25. Yoshimura H., T. Inoue, T. Yamada, K. Shiraki. Anemia during hard physical training (sports anemia) and its causal mechanism with special reference to protein nutrition. World Rev Nutr Diet 35: 1-86 1980. 


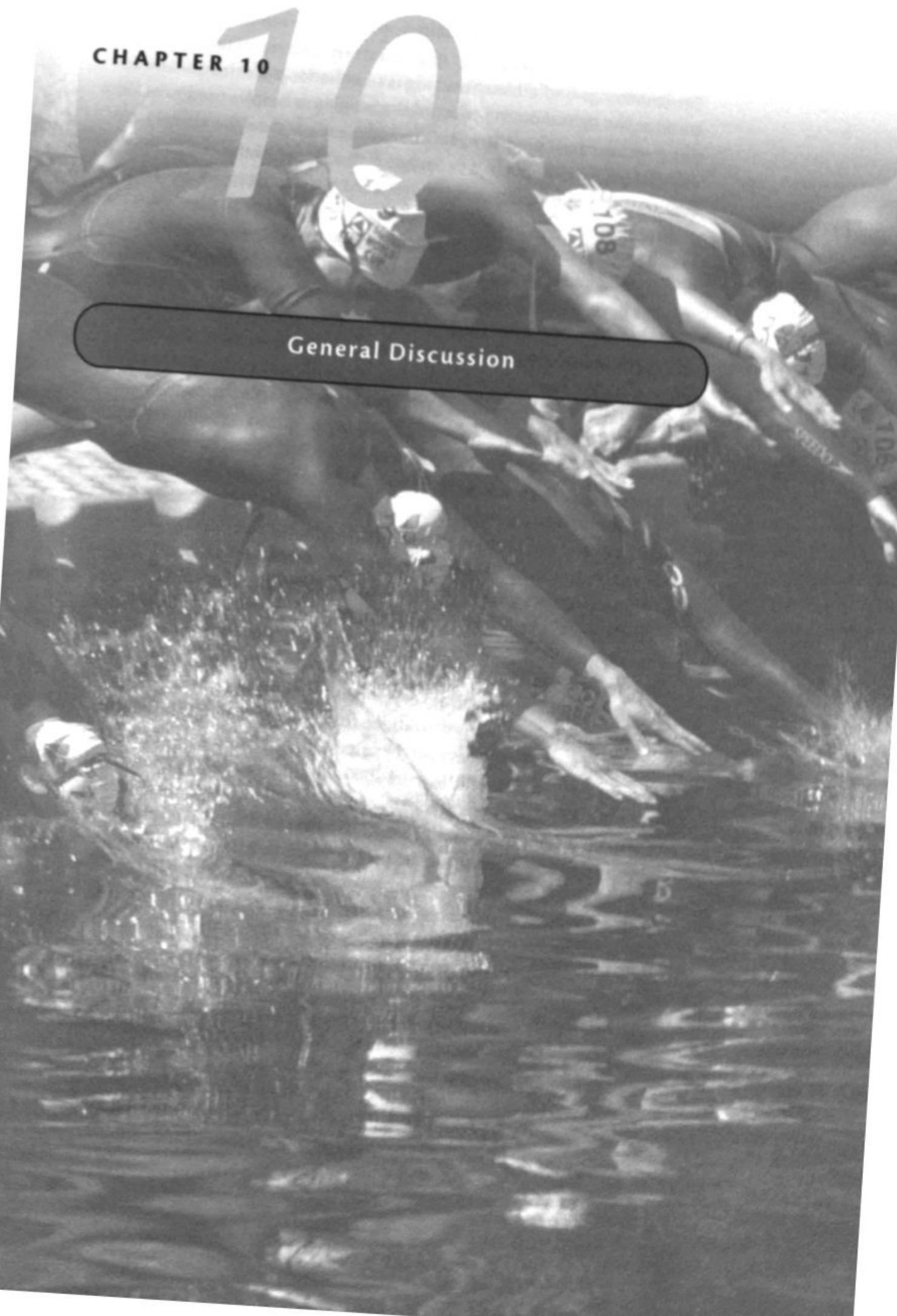


\section{Monitoring training adaptation}

The primary goal of athletic training is to enhance performance and to 'peak' at the right moment. Most of the training principles used to achieve this goal are based on many years of experience and on the model that physical exercise results in a variety of disturbances of the body's internal environment followed by recovery-induced alterations to adjust the body to the given exercise load. Although the scientific basis of physical training is still fragmentary, over the years, sport scientists have endeavoured to understand and describe the principles of physical training. Various studies have described key variables that may play an important factor in endurance performance (high maximal oxygen uptake, high gross mechanical efficiency, the ability to use fat as fuel during exercise at a high intensity), by using all kinds of diagnostic tools and exercise protocols. Most of the work done in this field concerns subjects that were not elite athletes and sometimes even untrained subjects and it is unknown whether all results obtained in less trained subjects can be extrapolated to elite athletes. Less is known about the physiological and biochemical effects of extensive periods of intensive training in well-trained elite subjects and how these adaptations correlate with performance.

For instance, it is well established that endurance exercise training results in numerous adaptations to metabolic, cardiovascular, respiratory and endocrine systems, eventually leading to an increased performance. An important question for sport practice is which of these diagnostic tools and exercise protocols are of value for monitoring training adaptations. Therefore the first aim of this study was to monitor and evaluate physiological and biochemical adaptations to physical training through the several phases of an one year training program in elite triathletes, preparing for a major event (Sydney 2000 Olympic games) by using general accepted diagnostic tests. For this aim elite athletes from the Dutch Olympic distance triathlon team were monitored over a period of one to three years preceding the Sydney 2000 Olympic games from which the results are described in the chapters two, three and four.

The athletes were subjected to several generally accepted laboratory - and performance tests (maximal workload test). During the performance tests, gas exchange and heart frequency was measured continuously.

Furthermore, blood for determination of lactate, red and white blood cell profile, endocrinological variables (Growth hormone, IGF-1, ACTH, cortisol, testosterone and TSH) was withdrawn at standardized times before and eventually during the performance tests. Monitoring all these variables over time in these elite triathletes revealed that, despite the considerable changes in training load over the year, in general no major alterations were found. From this point of view, one could seriously argue 
about the usefulness of monitoring endocrine variables for monitoring training adaptations and overtraining in sport practice.

From all measured biochemical variables only red blood cell count and lactate concentration during sub-maximal exercise showed a significant decrease throughout the year. The decline in plasma lactate concentration during sub-maximal exercise is therefore to be considered as a reflection of training adaptations. The changes in red blood cell count appear not directly related to changes in performance capacity. Although changes in hematological variables as often seen in endurance athletes do not directly reflect changes in training it is remarkable that in this study a large number of athletes did show values below to lower normal limit (chapter 5). This suggests that iron status may easily be compromised and emphasizes the important of monitoring hematological status in athletes. Therefore, for sport practice, it is advised to monitor hematological status on a regular basis.

A remarkable observation according to hematological variables was that there appears to be something like an altitude threshold above which significant alterations in hematological status arise. No changes in any of the measured variables were found after altitude training at moderate altitude $(1500 \mathrm{~m}-1850 \mathrm{~m})$. In contrast, we found that alterations in the hematological variables were elicited when the altitude training was performed at an altitude around 2600 meter. The existence of an altitude threshold is supported by a study of Chapman et al. (4). The alterations in the hematological variables as found in the present study were not reflected in changes in maximal performance or maximal oxygen uptake during laboratory testing. Taken into account that most of the altitude training centres in Europe are situated below the $2000 \mathrm{~m}$ it is doubtful if these locations bring an optimal benefit for the greater part of the athletes. As altitude training becomes more and more a common practice in sports it is well worth to find out an athletes individual altitude threshold in order to get an optimal benefit out of the altitude training. The absence of major changes in the hematological variables and all of the other measured variables in the present study appears to be in line with other studies. Although the number of comparable studies is limited, in similar studies done in elite endurance athletes no major changes in these variables were found either $(6,10,15,27)$.

Taken together, the findings of the present investigations shows that in elite Olympic distance triathletes no major changes in the mentioned variables occurred throughout the year. However other studies did report changes in the reported variables $(8,9,16,19,26)$. One of the plausible explanations for these contradictory results may be the selection of subjects in the various investigations because the investigated variables 
may behave differently in elite compared to less trained athletes. It appears that in studies in which no or minor changes in the mentioned variables were found mainly elite or very well trained athletes were involved, whereas in studies in which significant changes were observed no elite athletes participated. This indicates that extrapolation of results obtained in non-elite endurance athletes to elite endurance athletes should be cautioned. It is likely that well-trained elite athletes have reached their potential limit and are able to maintain this high performance level even during periods of reduced training, as shown in chapter 7 .

Another explanation that may underlay the absence of significant changes in the mentioned variables in the subjects of the present study is that the methods used were not sensitive enough to measure possible small differences that may have been induced by the training in these elite athletes. Unfortunately there are just few studies that have examined the reproducibility of all the methods used in the present study. One of the generally used performance indices is $\mathrm{VO}_{2} \max$. It has been described that $\mathrm{VO}_{2}$ max measurements have an accuracy of 3-5\% (1) although modern equipment may have a higher accuracy around $1 \%$ (18). Not only the measurements have a given variability, also the subjects appear to have certain variability in performance. Kuipers et al (11) showed that the coefficient of variation (CV) for a maximal workload test varied between individuals from $4.2 \%$ to $11.35 \%$ and for maximal oxygen uptake the individual CV was between $2.95 \%$ and $6.38 \%$. A more recent study by Jeukendrup et al (7) showed CV values of $3.5 \%$ and $3.6 \%$ in a time trial test. Therefore it is quite possible that small changes in performance and blood chemical indices did occur in the athletes, but that these changes are within the range of normal variability of the used methods. Another factor that could have contributed to absence of positive changes in the performance tests is travelling, since Reilly et al. showed that passing time zones may induce deterioration of performance (17). Since the athletes in the present study frequently travelled this may have outbalanced training effects.

From the previous findings, it can be concluded that, with a few exceptions (for instance lactate and red blood profile) comprehensive biochemicaland endocrinological I testing in elite athletes on regular basis is at least questionable.

As it was found that elite athletes show a very stable pattern in biochemical, endocrinological and performance tests variables over the year, one could say that changes in one of these variables may indicate a possible disturbance of the training adaptation. Therefore, it is important to assess on a regular basis a blue-print of an athlete in optimal shape, which can be used as his/her golden standard. 


\section{Preventing overtraining}

A second important aim of this thesis was to monitor physiological, psychological and biochemical parameters, to provide data on early recognition of training maladaptations and to prevent athletes from overtraining. For this purpose, besides the most common measurements used in overtraining (-research) (among others, performance tests, hormones and questionnaires), new methods and variables were studied (i.e. cognitive speed test and shorted POMS list).

The magnitude of the adaptive training response depends on, and might be proportional to the product of intensity, volume and frequency of the training. However, it is still unknown what the optimal combination of these determinants is, and how much training is necessary for an optimal performance enhancement. Highly motivated athletes often challenge the delicate balance between optimal training and overtraining and unintentionally ignore early warning signs of overtraining and consequently continue their hard training. For the coach and the athlete it is very difficult to provide the optimal training load based only on subjective perception. Therefore in sport practice many clinical- and field measurements have been developed to measure training ( $\mathrm{mal}$ ) adaptations in order to allow the coach and athlete objectively make training programmes that maximise performance and keep the athletes away from overtraining. In the last decades many studies have been done to unravel the aetiology of overtraining and to find markers suitable to diagnose overtraining in athletes suffering mainly from unexplained performance deteriorations. This resulted in a large number of physiological parameters that have been claimed to identify overtraining (table 1).

The existing literature, however, is inconsistent and sometimes contradictory and mostly focused on a single parameter. Possible factors underlying this discrepancy in research findings are the use of different definitions for overreaching and overtraining, differences in subjects and differences in sports disciplines. Unfortunately little research has addressed severe overtraining because there are ethical considerations and limitations in intentionally overtraining subjects and it is debatable whether the subjects in the several studies that have been done were really overtrained.

Nevertheless, it is more desirable to keep athletes away from overtraining, because a state of overtraining causes mostly weeks to months of forced rest. Therefore, we have selected an alternative group of athletes to study this phenomenon. Variables often used in relation to overtraining are hormones and numerous studies have addressed this topic $(2,12,14,22)$. 
TABLE 1

An overview of the major symptoms as described in the literature

(adapted from Fry et al (5))

PHYSIOLOGICAL/PERFORMANCE

- Decreased performance

- Inability to meet previously attained performance standards

- Recovery prolonged

- Reduced toleration of loading

- Difficulty in concentration at work/training

- Decreased maximum work capacity

- Loss of co-ordination

- Decreased efficiency/amplitude of movement

- Reappearance of previously corrected movement

- Inconsistency in rhythmical movements

- Reduced capacity of differentiation and correction technical faults

- Increased difference between lying and standing heart rates

- Abnormal T wave pattern in ECG

- Heart discomfort on slight exertion

- Changes in blood pressure

- Changes in heart rate at rest. exercise and recovery

- Increased frequency of respiration

- Decreased body fat

- Increased oxygen consumption at sub maximal workloads

- Increased ventilation at submaximal workloads

- Increased heart rate at submaximal workloads

- Shift of lactate curve towards the $x$-axis

- decreased evening post workout weight

- Chronic fatigue/feelings of heaviness

- Insomnia

- Night sweats

- Thirst

- Anorexia nervosa

- Loss of appetite

- Bulimia

- Amenorrhea/oligomenorrhea

- Delayed menarche

- Headaches and nausea

- Increased aches and pains

- Muscle soreness and tenderness

- Tendinostic complains

- Periosteal complains

- Muscle damage

- Elevated C-reactive protein

- Rhabdomyolysis
PSYCHOLOGICAL /INFORMATION PROCESSING

- Feelings of depression

- General apathy

- Decreased self esteem/Wrsening feelings of self

- Emotional instability

- Decreased muscular strength

- Sensitive to work and environmental stress

- Fear of competition

- Not eating well

- Changes in personality

- Decreased ability to narrow concentration

- Increased internal and external distrctibility

- Decreased capacity to deal with large amounts of information

- Gives up when going gets tough

IMMUNOLOGICAL

- Increased susceptibility to and severity of illness/cold/allergies

- Flu-like illnesses

- Unconfirmed glandular fever

- Minor scratches heal slowly

- Swelling of lymph glands

- One day colds

- Decreased functional activity of neurophils

- Decreased total Lymphocyte count

- Reduced mitgen response

- Increased eosinophil count

- Decreased proportional of null (non-T non B lymphocytes)

- Bacterial infection

- Reactivation of Herpes viral infection

\section{BIOCHEMICAL}

- Negative nitrogen balance

- Elevated basal metabolic rate

- Hypothalamic dysfunction

- Flat glucose tolerance curve

- Depressed muscle glycogen concentration

- Decreaed bone mineral content

- Decreased hemoglobin/serum iron, ferritin

- Mineral depletion: Zn, Ca, Al, Se, Cu etc

- Increased Ura concentrations

- Elevated cortisol levels/low testosterone

- Increased surum hormone binding globulin

- Testosterone to cortisol ratio less than $30 \%$

- Increased uric acid production 
It is well established that repeated exposure to a stressor, as for instance exercise, can result in decreased response of the hypothalamic-pituitary axis (HP axis) (28) and changes in response of the hormones of the HP axis have been described several times in overtraining studies. Therefore, the plasma levels of testosterone and growth hormone $(\mathrm{GH})$ (reflecting the anabolic activity) and adrenocorticotropic hormone (ACTH) and cortisol (reflecting the catabolic activity) are often considered as useful diagnostic tools for overtraining. One of the questions addressed in this thesis is whether the HP axis changes already occur during the phase of overreaching. The results as described in chapter 8 , however showed that only plasma cortisol level was decreased after a short period of intensified training and this was only found after challenging the hypothalamicpituitary adrenal axis (HPA axis) by releasing hormones. This finding suggests that the adaptive response of the HPA axis to short term intensified training load leads to a diminished cortisol secretion without an attenuated ACTH secretion. Similar findings were observed in over-trained horses (3) and in six weeks heavily trained cyclists (13). However there are some important differences between these studies and our study. It was shown by Vasankari et al (23) that a single exercise bout of four hours resulted in an increase of the cortisol release and a decreased growth hormone release in non-overtrained healthy cyclists after stimulating with a releasing hormone. After an intensified training period of 6 weeks Lehman et al found a decrease in cortisol and testosterone release and an increase in ACTH and TSH release (13) after stimulating with a releasing hormone. However, Barron et al found in four runners, who were considered as overtrained, a decreased cortisol release after insulin injection resulting in hypoglycaemia (2). In the present study a decreased release of cortisol was found in seven cyclists after two weeks of intensified training after stimulating the HPA axis with a human cortico-releasing hormone (hCRH) but no changes were found after an insulin stimulated hypoglycaemia. These differences in outcome between the several studies are probably the result of the differences in the stage between normal training adaptation and overtraining" of the subjects in these studies.

Taken this into consideration, we hypothesize that the time sequence of hormonal responses to hard training, overreaching and overtraining is as follows: Firstly, the sensitivity of the adrenal cortex to ACTH decreases resulting in a decreased cortisol release. Secondly, as a consequence, the hypothalamic-pituitary system tries to compensate with an increased ACTH release and lastly, the pituitary becomes insensitive to cortisol releasing hormone, as has been described in the stress literature (20). In the first stage, an athlete shows this reaction only on a homological stressor (in our case hCRH), in the last stage an athlete will show this reaction also on a heterological stressor like hypoglycaemia (fig 1 ). 


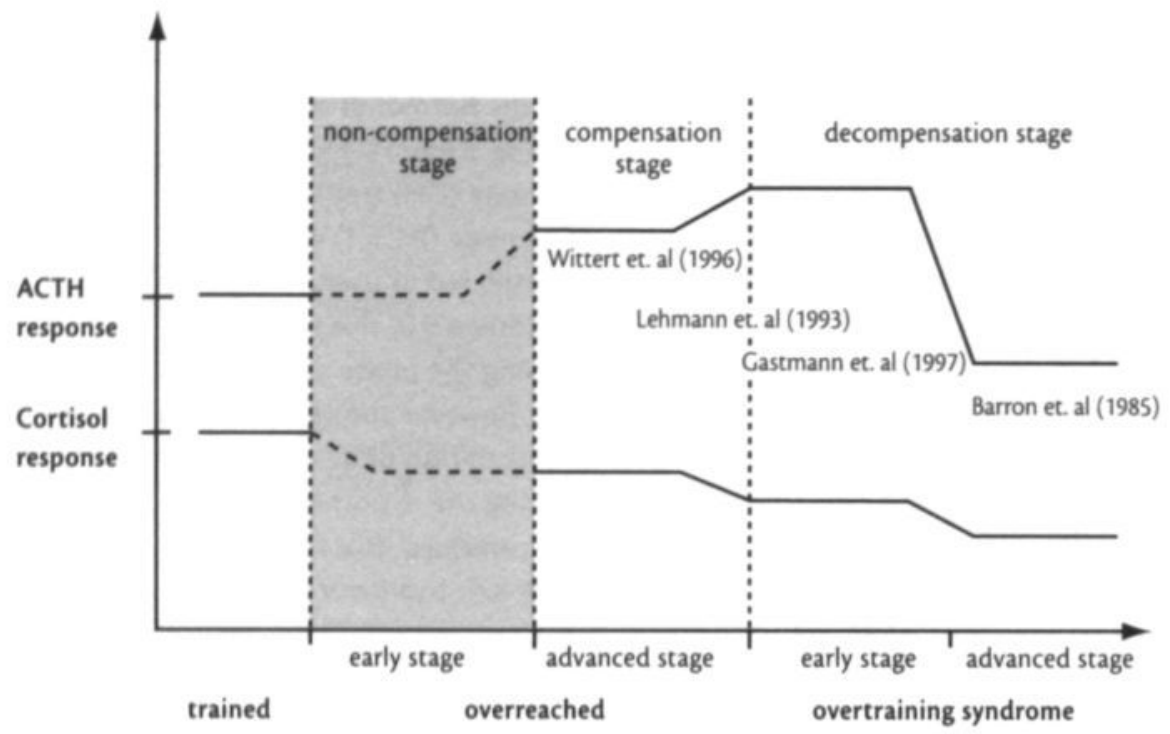

\section{FIGURE 1}

In the present study it was found that in an early stage of overreaching (gray area) cortisol decreases without an compensated increase in het ACTH release as found in the advanced stage of the overreaching as found byWittert et al (1996). Figure adapted and modified from Lehman et al. Overload, Performance Incompetence and Regeneration in Sport, (1999).

An important finding of the studies as described in the chapters 8 and 9 was that there was no relation found between changes in hormone release and any of the other variables measured during these two studies.

However, we must stress, that the hormonal responses to exercise and releasing hormones appeared to be extremely variable among subjects, and also within subject over time, as was described by Viru at al. $(24,25)$ and to make hormones useful as a tool for preventing or diagnostics overtraining it should be at least measured several times and under strictly standardized conditions.

Consequently, it can be challenged whether measurements of several hormones as generally done, are useful to detect overreaching or overtraining in its earliest stage. From the results of our studies and the results of the majority of other studies in this field (for an overview see Steinacker at al. (21)) it appears that from all hormones, only cortisol may be used in diagnosing overtraining. 
A novel finding of the present study was that cognitive tests demonstrated significant changes in the early phase of overreaching. This finding has not been described before and appears to offer possibilities to detect overtraining in an early stage. Although cognition appears to be sensitive to too much stress, among them a very intensified training period, the explanation for the observed changes is still unknown and further studies are needed to explore this approach to monitor training and overtraining. A question addressed in chapter 9 is whether a strenuous training program, which is supposed to induce overreaching, elicits the same response in different types of sport. Comparing the reaction of cyclists and runners to a period of intensified training it was shown that runners and cyclists do react differently according to their subjective perception of the training work. It was found that runners do react more emphatic.

This manifested itself in a higher RPE and POMS score. It was, interesting to observe that this was not paralleled by blood chemical or endocrinological variables. Despite the more pronounced feeling of fatigue and diminished vigor, runners maintained their performance level while the cyclist showed a decrease in performance. However, the runners showed an increased incidence of upper respiratory disease in the tapering phase. The lessons learned from our studies is that, RPE and POMS scores are very valuable tools to detect, already at an early stage when no biochemical changes can be found, a too large increase in training load, especially in impact sports like running.

\section{Summary of the main findings}

In this thesis the physical and performance characteristics from elite male and female triathletes were studied. It was found that during a season virtually no fluctuation was found in $\mathrm{VO}_{2} \max$, maximal power output, and other performance indices. Only plasma lactate response during submaximal exercise showed a rightward shift during competitive season, when plotted as function of workload. This would imply that when changes, especially decrements in performance indices, are found, this may indicate disturbance of normal training adaptation.

Haematological monitoring of the elite athletes showed that regular measurement of haematological variables is useful, because haemoglobin levels tend to be at the lower end of the normal range, with a risk to become anaemic. In this study no difference in haematological status between runners and cyclists were found. Therefore for sport practise regular measurements of haemoglobin and ferritin levels are indicated, whereas also moderate iron supplementation is recommended, especially in female athletes. 
An important finding in the present study is that for haematological adaptations to altitude training an altitude threshold appears to exist, below which no significant alterations in mean haematological status occurs. The threshold above which changes in haematological status can be observed, appears to be above an altitude of 1900 meters. In sport practice hormones are often measured to monitor training adaptations and to prevent overtraining. The data of the present study show that over a whole season virtually no changes in any of the hormones occur. Even in situations of intentional overtraining in general no changes occur in hormone status, except for plasma cortisol levels, that showed a decline during overtraining as response to a combined anterior pituitary challenge test. However, cortisol levels in the resting state and after maximal exercise failed to show changes during strenuous training. For sports practise this implies that repeated hormone measurements in the resting state contributes little to follow training adaptation and to prevent overtraining.

In one of the training studies it was found that a 21 days period of reduction in training, did not lead to a drop in performance. This finding appears to have important consequences for sport practise, where athletes are often afraid to lose conditioning with short periods of reduced training. This study shows that a few weeks reduction in training will not result in performance deterioration.

For assessment of performance capacity the lactate response to exercise is often used. However, the protocols for describing the lactate response vary considerably, and the duration of stages in incremental tests varies from 1 to 10 minutes. In this thesis the effect of stage duration on plasma lactate response and other physiological variables was measured. It appears that for measuring peakVO $\mathrm{O}_{2}$ and maximal heart rate a stage duration of one minute is sufficient. However, for lactate measurements a steady plasma concentration is reached only after at least 5 minutes. Thus for assessing performance capacity using lactate variables a stage duration of at least $\mathbf{5}$ minutes is required. The present study shows that the value of lactate measurements for predicting competitive speed is limited.

An important aim of the present study was to find markers for overtraining. The results of the present study showed that overtraining is not reflected in significant changes in haematological, endocrine and performance indices. It appears that the subjective feelings, scored with a Profile of mood state (POMS), and rate of perceive exertion(RPE) are more sensitive to overtraining than physiological measurements, with the exception of cortisol. The adrenal cortex was less responsive to a stimulus of adreno cortico tropic hormone (ACTH) during overreaching. 
It was also found that a cognitive test is a sensitive tool to detect early signs of to increased training loads. For sport practise this implies that it is important to have athletes register their profile of mood state and the rate of perceived exertion on a regular basis. In addition cognitive tests may be useful for early detecting of overtraining.

\section{Future recommendations}

A major problem in the world of sport science is that almost all laboratories use different training and testing protocols. In addition there is a lack of universal terminology used for all kind of general accepted terms. For instance, in overtraining studies, overtraining is described differently as overtraining syndrome, staleness, burn out, overstrain and overreaching and in spite of the absence of a golden standard to label someone as been overtrained, in all kind of studies subject are labelled as overtrained. It would therefore be desirable to come to more standardised definitions, protocols and more cooperation between laboratories in order to unravel the secrets of successful training (adaptation).

For future studies it is recommended to standardize the terminology of overtraining. Since it appears that burn out has similarities with overtraining as far patho-physiology is concerned, it should be explored whether more can be learned about overtraining from investigations in burnout patients and the other way around.

Furthermore it is recommended to explore the potentials of cognitive tests for monitoring the transition from reaching to overreaching, and for studying cognitive function in athletes suffering from an overtraining syndrome. And again, for this it is important to reach an uniform testing protocol that can be used in different centres in a unified way. 


\section{Reference}

1. Astrand P.O., K. Rohdahl. Textbook of work physiology. New York, London: MacGraw Hill International Editions, 1986.

2. Barron G.L., T.D. Noakes, W. Levy, C. Smith, R.P. Millar. Hypothalamic dysfunction in overtrained athletes. J Clin Endocronilogy and Metabolism 60: 803-806 1985.

3. Bruin G., H. Kuipers, H.A. Keizer, G.J. Vander Vusse. Adaptation and overtraining in horses subjected to increasing training loads. J Appl Physiol 76: 1908-1913 1994.

4. Chapman R.F. J. Stray-Gundersen, B.D. Levine. Individual variation in response to altitude training. J Appl Physiol 85: 1448-1456. 1998.

5. Fry R.W., A.R. Morton, D. Keast. Overtraining in athletes. An update. Sports Med 12: 32-65. 1991.

6. Hakkinen K., K.L. Keskinen, M. Alen, P.V. Komi, H. Kauhanen. Serum hormone concentrations during prolonged training in elite endurance-trained and strength-trained athletes. Eur J Appl Physiol Occup Physiol 59: 233-238 1989.

7. Jeukendrup A., W. Saris, F. Brouns, K. ADM. A new validated endurance performance test. Medicine and Science in Sports and Exercise 28: 266-270 1996.

8. Kiens B. Effect of endurance training on fatty acid metabolism: local adaptations. Med-Sci-Sports-Exerc 29: 640-645 issn: 0195-9131 1997.

9. Kiens B., B. Essen Gustavsson, N.J. Christensen, B. Saltin. Skeletal muscle substrate utilization during submaximal exercise in man: effect of endurance training. J Physiol Lond 469: 459-478 1993.

10. Kohrt W.M., J.S. O'Connor, J.S. Skinner. Longitudinal assessment of responses by triathletes to swimming, cycling, and running. Med Sci Sports Exerc 21: 569-575 1989

11. Kuipers H., F.T.J. Verstappen, H.A. Keizer, P. Geurten, G. Kranenburg van. Variability of Aerobic Performance in the Laboratory and Its Physiologic Correlates. Sport Med 6: 197-201 1985.

12. Lehmann M., C. Foster, H.H. Dickhuth, U. Gastmann. Autonomic imbalance hypothesis and overtraining syndrome. Med Sci Sports Exerc 30: 1140-1145 1998.

13. Lehmann M., K. Knizia, U. Gastmann, K.G. Petersen, A.N. Khalaf, S. Bauer, L. Kerp, J. Keul. Influence of 6-week, 6 days per week, training on pituitary function in recreational athletes. Br/ Sports Med 27: 186-192 1993.

14. Lehmann M., H. Wieland, U. Gastmann. Influence of an unaccustomed increase in training volume vs intensity on performance, hematological and blood-chemical parameters in distance runners. / Sports Med Phys Fitness 37: 110-116. 1997.

15. Mujika I., J.C. Chatard, S. Padilla, C.Y. Guezennec, A. Geyssant. Hormonal responses to training and its tapering off in competitive swimmers: relationships with performance. Eur / Appl Physiol Occup Physiol 74: 361-366 1996.

16. Ready A.E., H.A. Quinney. Alterations in anaerobic threshold as the result of endurance training and detraining. Med Sci Sports Exerc 14: 292-296 1982.

17. Reilly T., G. Atkinson, J. Waterhouse. Travel fatigue and jet-lag. / Sports Sci 15: 365-369. 1997.

18. Rietjens GJ., H. Kuipers, A.D. Kester, H.A. Keizer. Validation of a computerized metabolic measurement system (Oxycon-Pro) during low and high intensity exercise. Int / Sports Med 22: 291-294. 2001. 
19. Roca )., A.C. Agusti, A. Alonso, D.C. Poole, C. Viegas, J.A. Barbera, R. Rodriguez Roisin, A. Ferrer, P.D. Wagner. Effects of training on muscle $\mathrm{O}_{2}$ transport at VO $_{2}$ max. J Appl Physiol 73: 1067-1076 1992.

20. Sapolsky R.M., L.C. Krey, B.S. McEwen. Stress down-regulates corticosterone receptors in a site-specific manner in the brain. Endocrinology 114: 287-292. 1984.

21. Steinacker J.M., M. Kellmann, O. Bohm, Y. Liu, A. Opitz-Gresss, K.W. Kallus, M. Lehmann, D. Altenburg, W. Lormes. Clinical findings and parameters of stress and regeneration in rowers before world championships. In: Overload. Performance Incompetence and Regenration in Sport, edited by M. Lehmann, C. Foster, U. Gastmann, H. A. Keizer and J. M. Steinacker. New York: Kluwer Academic/Plenum Publishers, 1999, p. 71-80.

22. Urhausen A., H.H. Gabriel, W. Kindermann. Impaired pituitary hormonal response to exhaustive exercise in overtrained endurance athletes. Med Sci Sports Exerc 30: 407-414 1998.

23. Vasankari T.J., U.M. Kujala, S. Taimela, I.T. Huhtaniemi. Pituitary-gonadal response to gonadotropin-releasing hormone stimulation is enhanced in men after strenuous physical exercise. Acta Endocrinol (Copenh) 129: 9-14. 1993.

24. Viru A., K. Karelson, T. Smirnova. Stability and variability in hormonal responses to prolonged exercise. Int J Sports Med 13: 230-235 1992.

25. Viru A., M. Viru. Evaluation of endocrine activities and hormonal metabolic control in training and overtraining. In: Overload, Performance Incompetence and Regenration in Sport, edited by M. Lehmann, C. Foster, U. Gastmann, H. A. Keizer and J. M. Steinacker. New York: Kluwer Academic/Plenum Publishers, 1999.

26. Westgarth Taylor C., J.A. Hawley, S. Rickard, K.H. Myburgh, T.D. Noakes, S.C. Dennis. Metabolic and performance adaptations to interval training in endurance-trained cyclists. Eur / Appl Physiol 75: 298-304 1997.

27. White J.A., G. Quinn, M. Al-Dawalibi, J. Mulhall. Seasonal changes in cyclists' performance. Part I. The British Olympic road race squad. Br / Sports Med 16: 4-12. 1982.

28. Wittert G.A., J.H. Livesey, E.A. Espiner, R.A. Donald. Adaptation of the hypothalamopituitary adrenal axis to chronic exercise stress in humans. Med Sci Sports Exerc 28: 1015-1019 1996. 


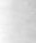




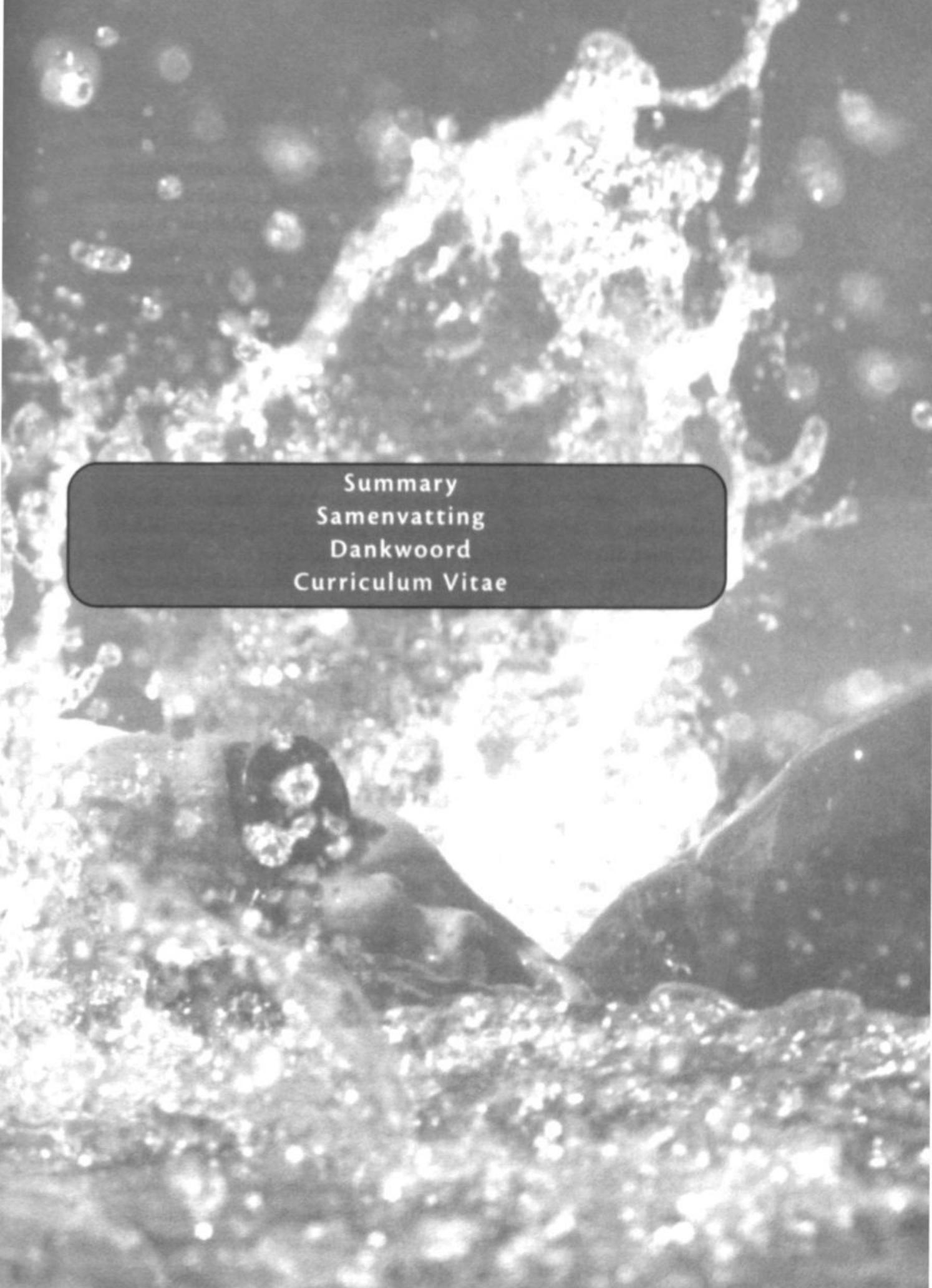




\section{Summary}

For most elite athletes winning an Olympic gold medal is the ultimate dream. To make this dream come true, in the first place one needs sufficient talent. However next to this talent, several years of training with large amounts of strenuous work is necessary. It is therefore not remarkable that the time required for adequate recovery may easily be compromised. Doing so, an athlete is often challenging the optimal balance between exercise and recovery.

The general purpose of this study was to get more understanding in the physiological adaptation processes of highly trained elite endurance athletes during periods of strenuous training.

This thesis is divided into three parts. The first part (chapter 2, 3 and 4) describes the results of a three-year longitudinal study in professional Olympic distance (OD) triathletes in preparation for Sydney 2000 Olympic games. Chapter 2 gives a description of some measured performance related physiological- and anthropometric variables in OD triathletes during the different phases of a year. Despite a clear difference in the amount of training between the several phases during the year, no significant differences were found between the measured performance related variables during submaximal and maximal exercise intensities. In spite of large amounts of training elite $O D$ triathletes have still a lower maximal oxygen uptake $\left(\mathrm{VO}_{2}\right.$ max $)$ compared to elite distance runners and elite cyclists. Chapter 3 describes the changes in the hematological blood profile, as a result of endurance- and altitude training. As red blood cells play an important role in the transport of oxygen to the working muscles, it is important to get insight into the dynamics of the red blood profile and its possible changes in athletes, for instance with very intensive training and altitude training. Over a three-year period, in a group of elite male and female OD triathletes' blood samples were collected during several moments throughout the year. It was found that intensive endurance training had not much influence on the red blood cell profile in male and female OD triathletes. However, it was found that most of the athletes examined (female even more than male athletes), had hematological values (particularly hemoglobin and ferritin) close to or in some cases below the lower limit of the normal range. Altitude training did alter the red blood profile significantly only when altitude training was performed above 2000 meters above sea level. In chapter 4, the effects of endurance training on the endocrine system throughout a year in $O D$ triathletes are described. Changes in hormonal plasma values are often mentioned as good indicators for the balance between training and 
recovery. Despite the significant differences in the amount of training between the different phases of a competitive season, no significant changes were found in the measured hormones throughout the year in elite male and female triathletes.

In the second part (chapter 5 and 6) some exercise protocols that are generally used to evaluate training and performance are addressed. In chapter $\mathbf{5}$ (cycling) and chapter $\mathbf{6}$ (running), the effect of stage duration on physiological variables in an incremental exercise test was studied. For measuring reliable lactate values during an incremental cycling exercise tests a stage duration of at least 5 minutes has to be taken into account. In a running protocol, the stage duration for obtaining blood lactate values that match exercise intensity, should be at least 6 minutes. When measuring maximal values such as peak $\mathrm{VO}_{2}$, peak heart rates or maximal workload incremental protocols with increments every minute are sufficient.

In the third part (chapter 7 to 9) training intervention studies are described. The purpose of these studies was to get more insight into the physiological changes of the human body during periods of imbalance between training load and recovery. Chapter 7 describes the effects of a $50 \%$ reduction in training load over a period of 21 days on some performance-related variables. Many trainers and athletes are afraid to reduce the training as they think this will cause a loss in performance. However, the results described in this chapter show that this fear is not supported by evidence. Furthermore, it was found that an intermittent training program had no benefit above a long slow distance training program. In chapter $\mathbf{8}$, the results of a training intervention study (overreaching) are described. The main purpose of this study was to investigate whether the generally accepted overtraining markers are useful, and can be used as early markers for overreaching. The main outcome of this study was that simple psychological questionnaires appear to be a more indicative and sensitive markers for detecting overreaching/overtraining in an early stage compared to the measured biochemical- endocrinological- and physiological variables. Furthermore, it was found that overreaching is associated with early changes in cognitive performance, which can be detected by simple cognitive performance tests. In chapter 9, possible differences in blood chemical, neuro-endocrine and psychological parameters in response to an intensified training period in runners and cyclists were investigated. The results of this study indicate that runners experienced a period of strenuous training as heavier compared to the cyclists. This difference of subjective feeling was not reflected in the measured blood chemical and neuro-endocrine variables. 
In conclusion, the most important findings in this thesis are:

- OD triathletes show hardly any variation in generally in sport measured biochemical-, hormonal-, or performance related variables.

- Regular control of the red blood profile is useful in well-trained endurance athletes as many of these athletes have blood values close to or below the lower limit of the normal range

- The results of this study indicate that there exists an 'altitude threshold' above which hematological alterations occur.

- The endocrine system is (with the exception of cortisol) not a good tool to prevent overtraining.

- Cognitive performance tests appear to be useful to detect overreaching in its earliest stage.

- Endurance athletes are able to maintain their performance level for some weeks in spite of reducing the training volume.

- The subjective perception of very intense training is different between cyclist and runners

- To ensure a reliable lactate level in incremental exercise tests, a stage duration of at least 5 minutes is required. 


\section{Samenvatting}

Voor het merendeel van de topsporters is het halen van een Olympische medaille de ultieme droom. Om deze droom te kunnen verwezenlijken dient de sporter op de eerste plaats over het nodige talent te beschikken. Maar naast talent zijn voor het bereiken van dit doel ook jaren van enorme hoeveelheden trainingsarbeid nodig. Het is dan ook niet verwonderlijk dat een topsporter zich voordurende begeeft op de dunne scheidslijn tussen trainingsbelasting en zijn trainingsbelastbaarheid. Daarbij wordt de grens van de belastbaarheid niet zelden overschreden. Het doel van deze studie was meer inzicht te krijgen in de veranderingen in fysiologische processen gedurende perioden van intensieve duurtraining bij zeer goed getrainde duursporters.

Dit proefschrift is dan ook onder te verdelen in drie delen. In het eerste deel (hoofdstuk 2, 3 en 4) worden de resultaten beschreven van een drie jaar durend longitudinaal onderzoek bij professionele Olympic distance $(O D)$ triatleten in voorbereiding op de Olympische spelen van Sydney 2000. In Hoofdstuk 2 wordt een beschrijving gegeven van verschillende prestatie bepalende fysiologische- en anthropometrische parameters gemeten bij professionele OD triatleten gedurende de verschillende fasen in een jaar. Ofschoon er duidelijke verschillen waren in de totale hoeveelheid verrichte trainingsarbeid tussen de verschillende fasen werden er geen significante verschillen gevonden in de gemeten fysiologische parameters tijdens submaximale en maximale inspanning. Olympic distance triatleten blijken ondanks de grote hoeveelheid trainingsarbeid echter een lagere maximale zuurstofopname $\left(\mathrm{VO}_{2} \mathrm{max}\right)$ te hebben in vergelijking tot professionele hardlopers en wielrenners. Hoofdstuk 3 beschrijft het verloop van het rode bloedbeeld profiel ten gevolge van duurtraining en hoogtetraining. Gezien de belangrijke zuurstof transporterende functie van de rode bloedcellen tijdens inspanning was het belangrijke inzicht te verkrijgen in de dynamiek van het rode bloedbeeld en de mogelijke veranderingen ten gevolge van zeer intensieve duurtraining en trainingsinterventies zoals hoogte training. Gedurende een periode van drie jaar zijn bij een groep professionele mannelijke en vrouwelijk OD triatleten op verschillende momenten in het trainingsjaar bloedmonsters afgenomen. Hieruit bleek dat intensieve duurtraining zowel bij mannelijke als vrouwelijke $O D$ triatleten niet van grote invloed was op het rode bloedbeeld. Wel bleek dat een groot deel van de geteste atleten (vrouwen meer nog dan mannen) met name hemoglobine en ferritine waarden hadden die dicht tegen de ondergrens aanlagen en in een aantal gevallen zelfs daaronder. Hoogtetraining liet alleen een significant verschil zien in het rode bloedbeeld indien het verblijf op hoogte boven de 2000 meter lag. In hoofdstuk 4 worden de trainingseffecten op het hormonale 
systeem beschreven bij OD triatleten gedurende een jaar. Hoewel veranderingen in hormonale plasma waarden vaak beschouwd worden als een goede reflectie van de balans tussen belasting en belastbaarheid, werden er in deze studie, ondanks significante verschillen in trainingsarbeid tussen de verschillende trainingsfasen in het jaar, geen significante verschillen gevonden in de gemeten hormonale variabelen. Dit gold zowel voor de mannelijke en vrouwelijke atleten.

In het tweede deel (hoofdstuk 5 en 6) wordt ingegaan op enkele testprotocollen die gebruikt worden voor de evaluatie van training en prestatie. In hoofdstuk 5 (fietsen) en hoofdstuk 6 (lopen) wordt gekeken naar de invloed van de tijdsduur van de belastingstappen op enkele fysiologische variabelen in een oplopend testprotocol op enkele fysiologische variabelen. De resultaten geven aan dat bij een fietstest een stap minimaal 5 minuten moet duren voor het verkrijgen van een betrouwbare lactaat waarde, die past bij de belasting. Bij een looptest is een stapduur van minimaal 6 minuten noodzakelijk. Voor het verkrijgen van een maximale waarden zoals een $\mathrm{VO}_{2}$ max of maximaal vermogen (Wmax) is een korter stapduur ( \pm 1 minuut) echter wenselijk.

In derde deel (hoofdstuk $7 \mathrm{t} / \mathrm{m}$ 9) worden tot slot enkele trainingsinterventie studies beschreven die als doel hadden meer inzicht te krijgen in de fysiologische veranderingen van het lichaam als de grens van belastbaarheid wordt overschreden. Hoofdstuk 7 beschrijft de effecten van een reductie in trainingvolume van $50 \%$ op een aantal prestatie bepalende variabelen. Veel trainers en atleten zijn bang dat een afname in trainingsarbeid al in korte tijd resulteert in een in afname van het prestatieniveau. De resultaten beschreven in dit hoofdstuk tonen echter aan dat deze angst onterecht is en dat een atleet in staat is zijn prestatieniveau op peil te houden gedurende 21 dagen ondanks halvering van het trainingsvolume. Daarbij blijkt het niet uit te maken of deze training een intermitterend of long slow distance karakter heeft. In hoofdstuk $\mathbf{8}$ worden de resultaten besproken van een overreaching studie. Hierin werd getoetst of de diverse in de literatuur beschreven overtraining parameters bruikbare indicatoren kunnen zijn in het vroegtijdig opsporen van overreaching, teneinde een overtrainingssyndroom te voorkomen. De voornaamste uitkomst in deze studie was dat een eenvoudige (psychologische) vragenlijst een gevoeliger instrument was in vergelijking met de duurdere biochemische-, hormonale- en fysiologische parameters voor vroegtijdige detectie van overreaching. Een belangrijke bevinding was dat een eenvoudige reactie taak een zeer gevoelig instrument bleek te zijn bij de detectie van overreaching. In hoofdstuk 9 wordt onderzocht of wielrenners en hardlopers verschillend reageren op een periode van zeer intensieve training wat betreft biochemische-, hormonale- en psychologische parameters. 
Hardlopers blijken een verdubbeling van hun trainingvolume veel zwaarder te ervaren in vergelijk met wielrenners. Dit subjectieve verschil in kwam echter niet tot uitdrukking in de verschillende gemeten biochemische- en hormonale variabelen.

Samengevat zijn de belangrijkste conclusies van dit proefschrift:

- OD triatleten laten nauwelijks enig schommeling zien in de veel in de sport gemeten biochemische-, hormonale- en prestatie bepalende fysiologische parameters.

- Regelmatige controle van het rode bloedbeeld is zinvol bij goed getrainde duuratleten, gezien het groot aantal atleten met bloed waarden dicht tegen of onder de minimum grens.

- Resultaten in deze studie duiden op het bestaan van een 'altitude threshold' waarboven hematologische veranderingen plaatsvinden.

- Het endocriene systeem (met uitzondering van cortisol) blijkt geen geschikt en bruikbaar instrument te zijn ter preventie van overtraining.

- Cognitieve testen zijn een gevoelig instrument voor de vroege opsporing van overreaching.

- Duurgetrainde atleten zijn ondanks halvering van hun trainingvolume in staat hun prestatieniveau te handhaven.

- Wielrenners en hardlopers reageren qua gevoel verschillend op een periode van intensieve training.

- Voor het verkrijgen van een lactaat waarde passend bij de belasting is bij fietsergometerarbeid een minimale inspanningsduur van 5 minuten noodzakelijk. 


\section{Dankwoord}

Vele malen heb ik tijdens duurloopjes, lange fietstochten of gewoon tijdens de dagelijkse autoritten tussen Kerkrade en Maastricht v.v. in gedachte het dankwoord geschreven. En steeds dacht ik dan, was ik maar zo ver dat ik het dankwoord kon schrijven want dat betekende dat het proefschrift voor het overgrote deel klaar zou zijn. ledere keer ook kwamen er nieuwe mensen bij die ik in dit dankwoord wilde noemen. Nu is het dan eindelijk zo ver dat ik het dankwoord kan schrijven, maar juist nu val ik een beetje stil en ben ik op zoek naar de juiste woorden, zinnen. Niet omdat ik opeens vind dat er uiteindelijk niemand meer een bedankje behoeft. In tegendeel zelfs want gedurende de afgelopen vijf jaar heb ik het genoegen gehad te werken met een groot aantal to atleten, toptrainers en topwetenschappers die allen op hun eigen wijze een zeer positieve bijdrage hebben geleverd bij het tot stand brengen van dit proefschrift. Aangezien de deadline voor het inleveren van dit stukje nog maar 24 uur verwijderd is, is het noodzaak toch maar gewoon te beginnen met schrijven.

Graag wil ik beginnen met Dr. Hans Keizer. Hans, jij was degene die mijn interesse in de inspanningsfysiologie al tijdens het eerste jaar van mijn studie Gezondheidswetenschappen heeft aangewakkerd en mij op het spoor van het wetenschappelijk onderzoek heeft gezet. Verder wil ik je ook bedanken voor je zeer aangename rol van reisgenoot tijdens de talrijke reizen die we samen hebben gemaakt in binnen- en buitenland. Jij wist op je eigen manier altijd weer een extra dimensie aan deze reizen toe te voegen. Jij zou dan ook de perfecte auteur zijn voor het schrijven van een Lonely Planet, met als titel "1001 oplossingen voor de gestrande reizige".. Hans, bedankt voor alles wat jij voor mij hebt gedaan. Ik heb enorme bewondering voor je als mens en wetenschapper en ik hoop dat onze samenwerking niet eindigt met het afsluiten van mijn aio-periode.

Prof. Harm Kuipers, ik ben jou zeer erkentelijk voor de onvolwaardelijke steun die jij me onder alle omstandigheden steeds hebt gegeven gedurende de afgelopen 4 jaren. Jouw rustgevende en relativerende inbreng in het geheel hebben een zeer grote bijdrage gehad in het welslagen van mijn aioperiode. Daarnaast ook dank voor de regelmatige 'lunchvergaderingen' die we al rennend hebben afgewerkt en de talloze vlieglessen.

Prof. Dr. Wim Saris, jij hebt op een geheel eigen wijze mij steeds weten te stimuleren het maximale eruit te halen. En hoewel ons contact niet zeer frequent was, heeft jouw kennis, visie en inbreng (je opmerkingen in de manuscripten waren altijd zeer opbouwend en constructief) een zeer positieve bijdrage geleverd bij het uiteindelijke eindresultaat van dit proefschrift. 
Ik ben er dan ook zeer trots op dat ik kan zeggen dat jullie mijn (co)promotoren waren.

Speciaal woord van dank gaat ook uit naar Louis Delahaye. Louis bedankt voor de manier hoe jij me hebt opgenomen in de triathlonwereld en me hebt laten kennis maken met de praktische kant van de topsport. Jouw kennis, manier van werken en de omgang met atleten heeft altijd indruk op me gemaakt. Zeker ook door de wijze waarop jij je wetenschappelijke achtergrondkennis integreert in de sportpraktijk. Ik heb onze samenwerking dan ook als zeer prettig ervaren en ik hoop dan ook dat we in de toekomst nog een keer de kans krijgen een vergelijkbaar project samen uit te voeren, met de daarin opgenomen onze plannen zoals wij die zo vaak hebben besproken. Daarom ben ik dan ook blij dat jij tijdens de verdediging van dit proefschrift als vriend en paranimf aan mijn zijde wil staan. Dit geldt ook voor mijn andere paranimf, Dennis van Hamont. Jij was meer dan een stagiaire alleen. Het werk (van kwalitatief zeer hoog niveau) dat jij verzet hebt gedurende meer dan twee jaren was onvoorstelbaar, zeker als je weet wat voor werkzaamheden je er nog allemaal naast deed. Toen jij naar Nijmegen vertrok voor je studie geneeskunde was ik dan ook niet blij. Gelukkig is ons contact er wel gebleven en mag ik je tot een van mijn vrienden rekenen.

Uiteraard ben ik ook dank verschuldigd aan de triatleten die iedere keer weer naar het verre zuiden kwamen om zich te onderwerpen aan een hele batterij testen. In willekeurige volgorde; Silvia, Wieke, Lucienne, Ingrid, Eric, Rob, Dennis, Huib, Raymond, Guido en Ralph HARTELIJK DANK voor jullie onverminderde inzet tijdens de testen, de interesse in mijn werk en het plezierig verblijf met jullie tijdens enkele trainingsstages.

Dank ook aan alle proefpersonen die zich steeds voor de volle honderd procent hebben ingezet om ieder experiment tot een succes te maken. Tijdens dit werk was ook de inbreng van de talloze studenten/stagiaires/ sportartsen i.o. onontbeerlijk. Kirsten, Dotje, Tim, Ingrid, Dennis, Michel, de 'Amsterdammers' Arie en Ronald, Rob V, Rob E, Paul, Robert en Wijnand. Ik heb me gelukkig mogen prijzen met zoveel inzet, motivatie en gezelligheid van jullie kant.

Een belangrijke bijdrage in mijn werk was ook de goede collegiale sfeer op de werkvloer. Allereerst mijn collegae (ex)AIO's van BW: Lars (60 meter zou Olympisch moeten worden), Marchel (als ik je keuken nog een keer moet verbouwen hoor ik het wel), Reinout, Ron, Ronnie, Annette, Miriam en Olivier; en de HB'ers: Freddy, Chris, Michiel, Edward, Erwin, Marco, Joost, Jogchum, Luc, Eva en Patrick.

Verder een speciaal woord van dank aan Matthijs, jij bent gedurende mijn gehele periode bij BW een steunpunt geweest waar ik altijd terecht kon met een probleem van wat voor aard dan ook. Eric, ook aan jou ben ik veel dank verschuldigd. Jij bent misschien wel 'schuld' aan mijn AIO baan. 
In ieder geval heb jij met jouw wetenschappelijke kennis een grote inbreng gehad in het geheel. Als kamergenoot was jij meestal als eerst de klos als iets niet goed dreigde te gaan, maar vrijwel iedere keer kwam jij wel met een oplossing aan dragen. Ook heb ik veel plezier beleefd aan de trainingsstudies die we samen hebben uitgevoerd.

Gerrit, voor mij ben je in vele opzichten de tovenaar van de afdeling. Jouw insight kennis in het wielrennen is ongelofelijk en meestal had je het "product" in een sappig gesprek al genoemd weken voordat de pers met een openbaring kwam. Veel plezier heb ik ook beleefd aan onze gezamenlijke eerste stappen binnen het professionele voetbal (toch anders als wielrennen hè). Wat voetbal betreft, Paul buiten die ene keer dan was "de trots van het Zuiden" toch altijd sterker dan Ajax. Ik wacht dan ook net zo als jij nog steeds op een aantal vlaaien van Hans, Gors en Olivier. Desiree, jij bedankt voor al de ad hoc oplossingen als ik iets vergeten was. Jos bedankt voor jou nieuwe inbreng in de overtraining studies en voor de correctie werkzaamheden. Fred bedankt voor de baan die je me destijds gegeven hebt, het is waarschijnlijk de aanloop geweest tot dit alles. Een leuke afwisseling van de dagelijkse werkzaamheden was vaak ook de onophoudelijke discussie over het meest optimale computerbesturingssysteem. Uit ervaring weet ik nu dat ze één ding gemeen hebben. Beide systemen kunnen vastlopen en doen dat bij voorkeur op die momenten dat je het absoluut niet kunt gebruiken. Daarom Harry en Leon (Windows) en Paul en Loek (Macintosh) bedankt voor al jullie hulp en oplossingen voor mijn computerproblemen. Dean, thanks for the English corrections. Joan, er komt vast nog een keer een moment dat we samen een kroeg opzoeken om samen een Belgisch biertje te drinken. Verder alle andere mensen binnen Bewegingswetenschappen, Humane Biologie en andere Capaciteitsgroepen die ik niet heb genoemd, bedankt voor jullie hulp en collegialiteit.

Jossie, dank voor alle lay out overuren die je hebt gemaakt.

Speciaal woord van dank ook nog aan de mensen van NOC ${ }^{*}$ NSF sector Topsport, in het bijzonder Frans van Dijk (en zijn voorganger Cees Vervoorn), Joop Alberda en Marleen van de Berg. Dankzij jullie heb ik enkele van mijn dromen kunnen waarmaken.

I would also like to thank Prof. Dr. Klaas Westerterp, Prof. Dr Jurchen Steinacker, Prof. Dr. Viru, Prof. Dr. Ger van der Vusse en Dr. Robert Jan Brummer for their critical evaluation of this thesis.

Dank ook aan mijn schoonouders, Herman en Elizabeth Alzer, die als dat nodig was steeds weer bereid waren even op Fleur te passen en het nooit erg vonden als ik weer eens later kwam dan afgesproken.

Pap en man, bedankt voor alle steun en vertrouwen die jullie altijd aan mij hebben gegeven. Dit werk is ook een beetje voor jullie en ik hoop dat jullie trots op mij kunnen zijn. 
Miriam, het schrijven van een proefschrift vergt veel energie en tijd. En die tijd ging dan vaak ten koste van de tijd die ik aan jou en Fleur kon besteden. Toch had jij hier altijd begrip voor. Vanaf het moment dat ik op het idee kwam alsnog aio te worden tot nu toe heb je mij gesteund en gemotiveerd in mijn werkzaamheden. Gelukkig wist jij me er ook regelmatig aan te herinneren dat er nog meer is dan werk alleen. Fleurtje, jij bent mijn kostbaarste bezit en ik hoop dat ik je net zo veel plezier kan geven als dat jij mij dagelijks geeft. Ik hou van jullie twee!

\section{Bedankt allemaal !"}




\section{Curriculum Vitae}

Gerard Rietjens was born on 22 June 1963, in Weert, the Netherlands. He completed secondary school (MAVO) at the Philips van Horne School in Weert in June 1980. In March 1981 he started training for nurse in the St. Jans Gasthuis in Weert. After receiving his degree in 1984, he worked at the intensive care unit. During this time, he followed the specialization for intensive care nurse (degree in 1985). In 1985, he started the specialization for operation room nurse (surgery) at the Hoge School Eindhoven (degree in 1987). Gerard worked as an operation room nurse in the St. Jans Gasthuis until June 1989. During that year, he passed the exams for a colloquium doctum at the Maastricht University. In September 1989, he started to study Movement Sciences at the Faculty of Health Sciences, Maastricht University. At the same time, he started to work part-time as a paramedic at the GGD in Maastricht. During his study Movement sciences he did two internships; from May to December 1993 at the department of Movement sciences, Maastricht University, Maastricht, the Netherlands and from April 1994 to October 1994 at the School of Physical Education, University of Otaga, Dunedin, New Zealand. After his graduation (MSC) in June 1995, he started to work as research assistant for the Netherlands' Center for Doping Affairs ( $\mathrm{NeCeDo}$ ). Here he participated in several doping related intervention studies to investigate the effects of Androgenic-anabolic steroid use in strength athletes. In 1997, he had the opportunity to start as a Phd-student, at the department of Movement Sciences, Maastricht University on a project to investigate Olympic endurance athletes in preparation for the oncoming Sydney 2000 Olympic games. The research performed during this period is described in this thesis. During the Sydney Olympic games, Gerard was a member of the technical staff of the Netherlands Olympic committee and Netherlands Sports federation (NOC $\left.{ }^{*} N S F\right)$ ). In December 2001, he accepted a part- time job as teaching assistent at the department of Movement Sciences, Maastricht University. In January 2002, Gerard was asked by the Koninklijke Nederlandse Atletiek Unie (KNAU) to participate in their technical staff and support their program in preparation towards the Olympic Games of Athens in 2004 on part-time basis. Since 2001, he is also part-time member of the technical staff of the professional soccer team MVV in Maastricht, member of the expert-panel of the Dutch Triathlon Federation (NTB) and member of the Netherlands Olympic Committee TECNET. 


\section{Publications}

\section{Journal Articles}

- Rietjens GJWM, Kuipers H, Verstappen FT, Schoenmakers H, Hofman G. Stage duration in incremental running tests, effects on physiological variables and lactate indices. Br/ Sport med 2002; accepted for publication.

- Rietjens G, Kuipers H, Hartgens F, Keizer HA. Red blood cell profile of elite Olympic triathletes. A thee-year follow up. Int / Sport med 2002; accepted for publication.

- Breda van E, GJWM. Rietjens, and HA Keizer. Factor-1 (IGF-1) and InsulinLike Growth Factor Binding Protein-3 (IGFBP-3) concentrations during rest and exercise. Nutrition (submitted), 2002.

- Rietjens GJWM, Kuipers H, Saris WHM, Keizer HA. A reduction in training volume and intensity during 21-days does not impair performance in cyclists. Br/Sports Med 2001:35:431-434.

- Rietjens GJ, Kuipers H, Kester AD, Keizer HA. Validation of a computerized metabolic measurement system (Oxycon-Pro) during low and high intensity exercise. Int / Sports Med 2001;22(4):291-4.

- Hartgens F, Van Marken Lichtenbelt WD, Ebbing S, Vollaard N, Rietjens G, Kuipers $\mathrm{H}$. Body composition and anthropometry in bodybuilders: regional changes due to nandrolone decanoate administration. Int / Sports Med 2001;22(3):235-41.

- Kuipers H, Rietjens GJWM, Verstappen FT, Vanruiten W, Koelewijn T. Physiological variables and lactate indices in incremental exercise tests with different stage duration. Br J Sport med 2001; submitted.

- Hartgens F, Marken Lichtenbelt Van WD, Ebbing S, Vollaard N, Rietjens GJWM, Kuipers $\mathrm{H}$. Androgenic-anabolic steriod-induced body changes in strength athletes. The Physician and sportsmedicine 2001;29(1):49-66.

- Hartgens F, Straaten van H, Fideldij S, Rietjens GJWM, Keizer HA, Kuipers H. Androgenic-anabolic steriods and human deltoid muscle fibers: difference between polydrug regimens and single drug administration. submitted for publication.

- Hartgens F, Rietjens GJWM, Keizer HA, Kuipers H, Wolffenbuttel BHR. Effects of androgenic-anabolic steriods on apolipoproteins and lipoprotein(a). submitted for publication.

- Rietjens GJWM, Kuipers H, Delahaye L, Keizer HA. Physiological characteristics of elite Olympic distance triathletes. Br J Sport med submitted.

- Rietjens GJWM, Kuipers H, Saris WH, Kester AD, Keizer HA. Hormonal changes throughout the year in elite male and female triathletes. $A$ one year survey. Br / Sport med submitted.

- Rietjens GJWM, Kuipers H, Adam JJ, et al. Physiological, biochemical and psychological markers of overreaching. Med Sci Sports Exerc submitted.

- Rietjens GJWM, Kuipers H, Klomp RW, Koops A, Keizer HA. Physiological and psychological variables respond different in competive cyclists and 
runners after a period of intensified training. submitted 2002.

- De Hon O, Hartgens F, Van Baak MA, Buisman LJRM, Rietjens GJWM. De invloed van een eenmalige toediening van een supratherapeutische dosis salbutamol op de longfunctie en duurprestatie van niet-astmatische sporters. Geneeskunde en Sport 1999;32(5):9-15.

- Eijsden Besselink E, Rietjens GJWM, Delnoy E. Ontmoeting tussen medicus en musicus. Revalidata 1993:18-25.

- Pilot P, Kuipers H, Pop P, Rietjens GJWM. Het chronisch vermoeidheidssyndroom en de rol van de fysiotherapeut. Fysio Actueel 1998;15:24-27.

- Rietjens GJWM, Keizer HA, Kuipers H. Changes in hormonal levels during the season in elite female tri-athletes. Presented at the fifth $I O C$ world congress, Sydney 1999.

- Rietjens GJWM, Wijnen J, Savelberg H. Does a lateral excursion of the pedal enhance cycling power output and economy of motion? submitted.

\section{Reports}

- Rietjens GJWM. Nutrition and Athletic Performance. First International Scientific Congress 2001, Edmonton, Canada. NOC*NSF publicatie, 2002

- Hartgens F, Rietjens GJWM, Haren van SF, Vogels T, Vrijman EN. Huisarts en Doping. Rotterdam: Nederlands Centrum voor Dopingvraagstukken/ TNO-PG, 1998.

\section{Abstracts}

- De Hon O, Hartgens F, Van Baak MA, Buisman LJRM, Rietjens G. Acute Inhalation of sulbutamol increases endurance performance in well-trained non-asthmatic athletes. Med Sci Sports Exerc 1999;31(5):S402.

- Rietjens GJWM, Hartgens F, Visser V, Van Baak MA, Kuipers H. The effect of salbutamol on lung function and exercise performance in endurance athletes. Med Sci Sports Exerc 1998;5(30):S323.

- Hartgens F, Rietjens GJWM, Cheriex E, et al. Effects of anabolic steriod on cardiovascular risk factors and the left ventricle in bodybuilders. Med Sci Sports Exerc 1997;5(29):S292.

- Rietjens GJWM, Keizer HA, Kuipers H. Validation of Oxycon Pro measurements during sub maximal and maximal exercise intesities. Med Sci Sports Exerc 2001; S suppl.(32):5317.

- Wolffenbuttel BHR, Hartgens F, Rietjens GJWM, Keizer HA, Kuipers H. Effects of androgenic-anabolic steriods (AAS) on apolipoproteins and lipoprotein (a). Presented at the annual meeting of the International Atherosclerosis Society, Paris 1997.

- Wolffenbuttel BHR, Hartgens F, Rietjens GJWM, Keizer HA, Kuipers H. Nandrolone decanoate reduces lipoprotein(a) levels without effect on other lipid parameters. Presented at the annual meeting of the International Atherosclerosis Society, Paris 1997. 



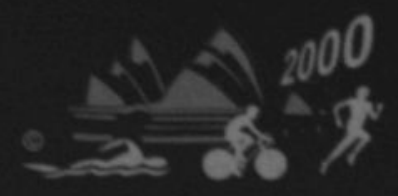

